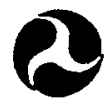

US Deparment

of Trassportotion

National Hogway

Troffic Sotety

Adrinistrotion

DOT HS 808078

$\therefore$

November 1993

Final Report

\title{
Marijuana and Actual Driving Performance
}

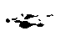

This document is availalbe to the public from the National Tectrical Intormation Service. Springfield. Virginia 22161 
This publication is distributed by the U.S. Department of Transportation, National Highway Traffic Safety Administration, in the interest of information exchange. The opinions, findings and conclusions expressed in this publication are those of the author(s) and not necessarily those of the Department of Transportation or the National Highway Traffic Safety Administration. The United States Government assumes no liability for its contents or use thereof. If trade or manufacturers' name or products are mentioned, it is because they are considered essential to the object of the publication and should not be construed as an endorsement. The United States Government does not endorse products or manufacturers. 
Technical Report Documentation Poge

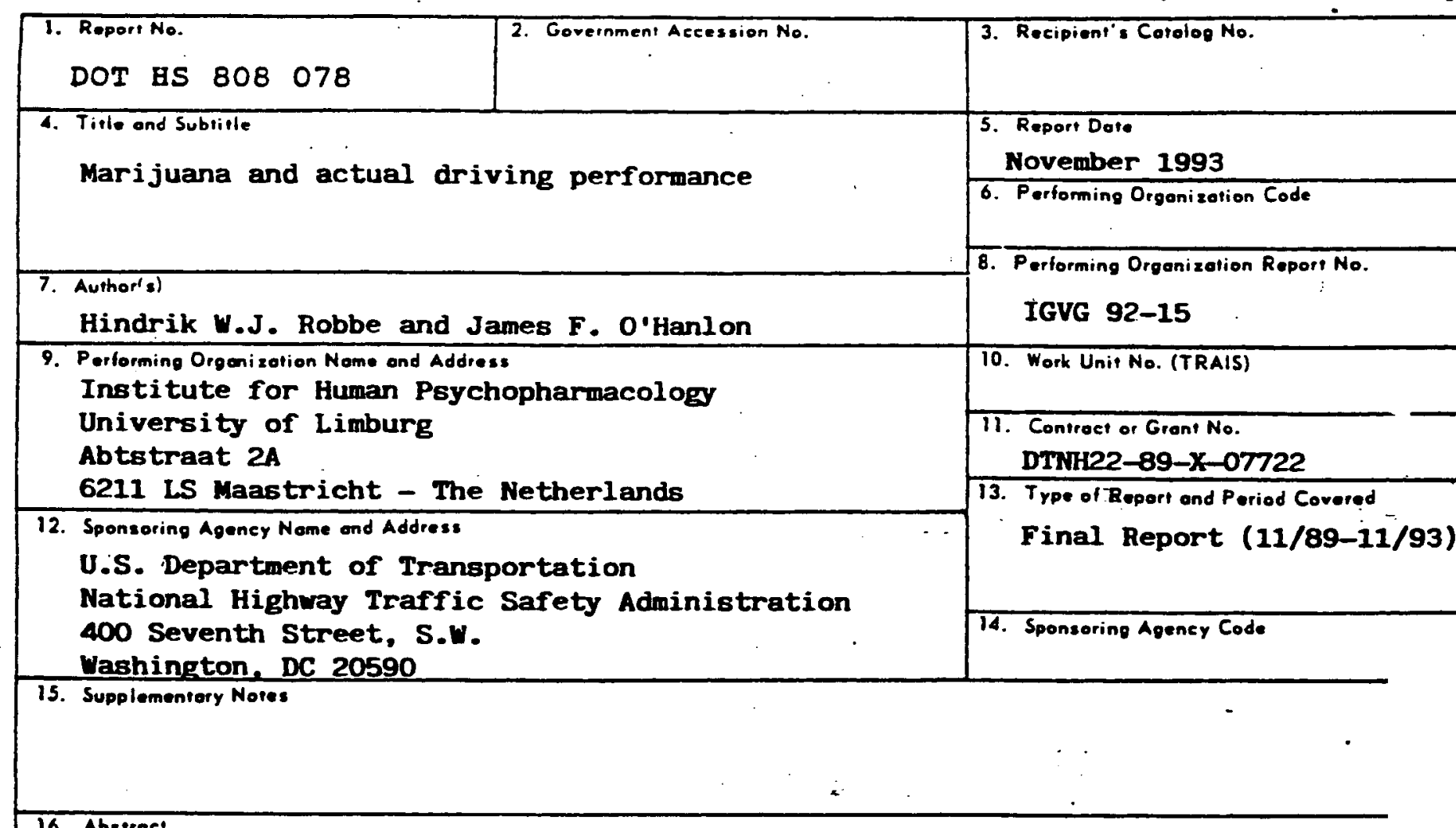

This report concerns the effects of marijuana smoking on actual driving performance. It presents the results of one pilot and three actual driving studies. The pilot study's major purpose was to establish the THC dose current marijuana users smoke to achieve their desired "high". From these results it was decided that the maximum THC dose for subsequent driving studies would be $300 \mu \mathrm{g} / \mathrm{kg}$. The first driving study was conducted on a closed section of a primary highway. After smoking marijuana delivering THC doses of $0,100,200$, and $300 \mu g / k g$ subjects drove a car while maintaining a constant speed and lateral position. This study was replicated with a new group of subjects, but now in the presence of other traffic. In addition, a car following test was executed. The third driving study compared the effects of a modest dose of THC (100 $\mu \mathrm{g} / \mathrm{kg}$ ) and alcohol (BAC of $0.04 \mathrm{g \%})$ on city driving performance. This program of research has shown that marijuana, when taken alone, produces a moderate degree of driving impairment which is related to the consumed THC dose. The impairment manifests itself mainly in the ability to maintain a steady lateral position on the road, but its magnitude is not exceptional in comparison with changes produced by many medicinal drugs and alcobol. Drivers under the influence of marijuana retain insight in their performance and will compensate where they can, for example, by slowing down or increasing effort. As a consequence, THC's adverse effects on driviog performance appear relatively small.

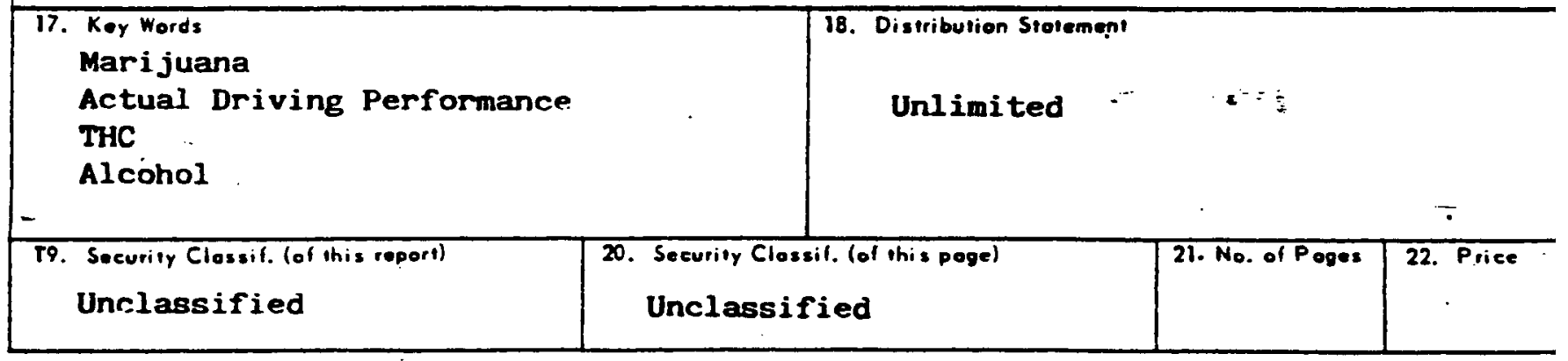

Form DOT F 1700.7 (8-72)

Reproduction of completed page authorized 


\section{CONTENTS}

List of Abbreviations $\ldots \ldots \ldots \ldots \ldots \ldots \ldots \ldots \ldots \ldots$

Acknowledgements $\ldots \ldots \ldots \ldots \ldots \ldots \ldots \ldots \ldots \ldots \ldots \ldots \ldots$

Executive Summary $\ldots \ldots \ldots \ldots \ldots \ldots \ldots \ldots \ldots \ldots \ldots \ldots \ldots$

Chapter 1 Marijuana and Driving: A Review . . . . . . . . . . . . . . . . . I

The Drug and Its Pharmacological Properties . . . . . . . . . . . . . 1

Prevalence of Marijuana Use .......................... 3

Marijuana and Traffic Safety ....................... 3

Epidemiological Research . . . . . . . . . . . . . . . . . . 3

Marijuana's Effects upon Driving Simulator Performance ........... 5

Marijuana's Effects upon Actual Driving Performance ............. 6

General Conclusion . . . . . . . . . . . . . . . . . . . . 9

Chapter 2 General Methods . . . . . . . . . . . . . . . . . . . . . 11

Description of a 4-Study Program . . . . . . . . . . . . . . . . 11

Subjects . . . . . . . . . . . . . . . . . . . . . . 12

Compliance with Ethical and Legal Standards . . . . . . . . . . . . . . . . . . 14

Screening for the Presence of Other Illicit Drugs and Alcohol . . . . . . . . . . . . 14

Blood Sampling and Quantitative Analyses . . . . . . . . . . . . . . 15

Chapter 3 Pilot Study to Select THC Doses . . . . . . . . . . . . . . . . . . 17

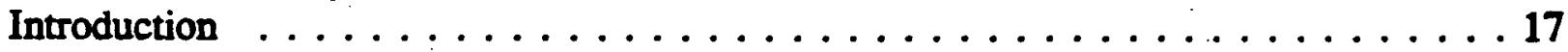

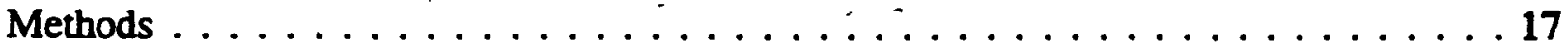

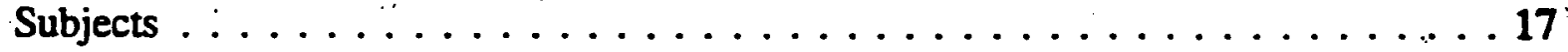

Smoking Procedures ........................ 18

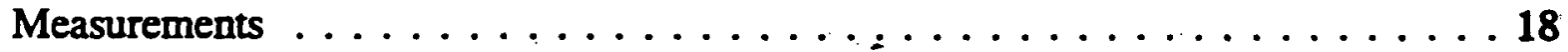

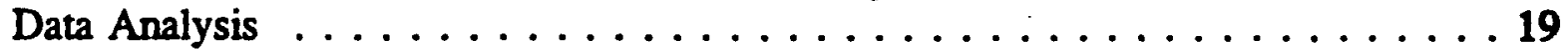

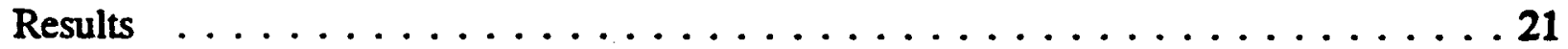

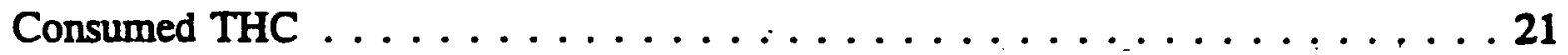

Plasma Concentrations of the Drug . . . . . . . . . . . . . . 21

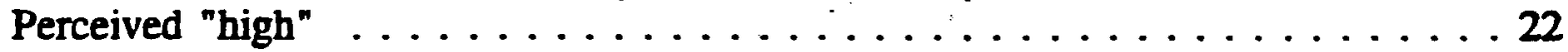

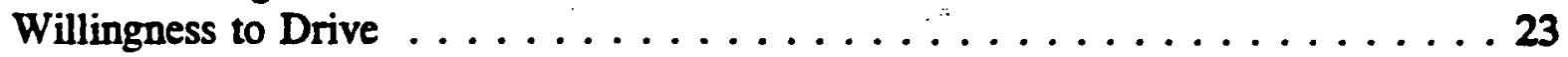

Perceived Alertness, Contentedness and Calmness . . . . . . . . . . . . . 24

Critical Tracking Test . . . . . . . . . . . . . . . . . . 24

Hand Steadiness Test . . . . . . . . . . . . . . . . 26

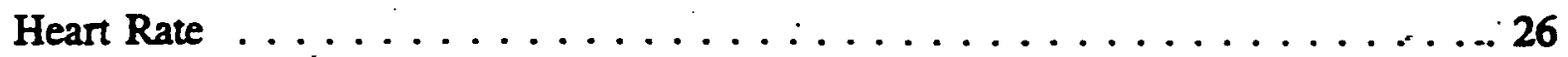

Intra-Subject Relations between Variables . . . . . . . . . . . . . . 26

Inter-Subject Relations between Variables . . . . . . . . . . . . . 28

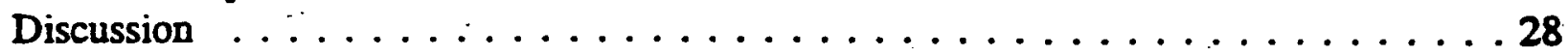


Chapter 4 Marijuana and Driving on a Restricted Highway $\ldots \ldots \ldots \ldots \ldots$

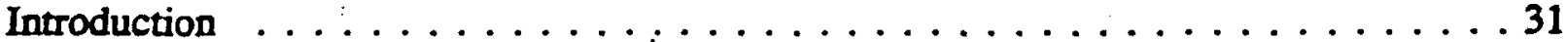

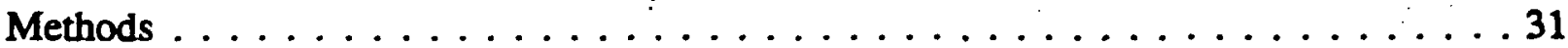

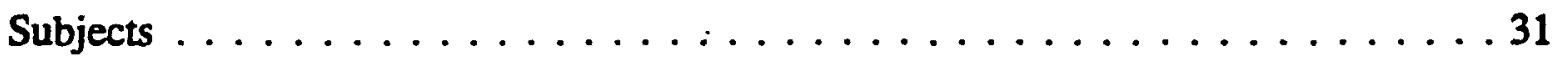

Design, Doses and Administration . . . . . . . . . . . . 31

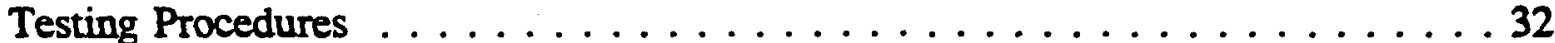

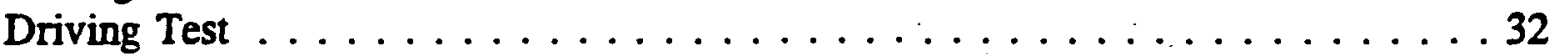

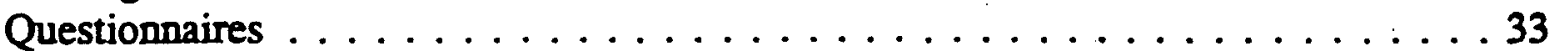

Laboratory Tests . . . . . . . . . . . . . . . . . . . 33

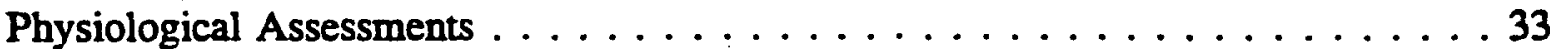

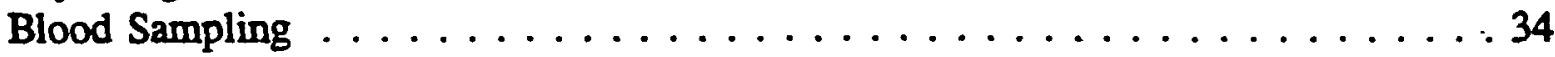

Data Analysis . . . . . . . . . . . . . . . . . . . 34

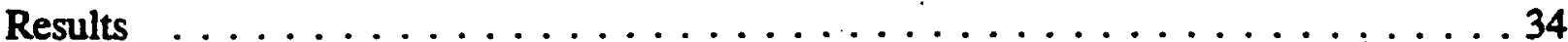

Plasma Concentrations of the Drug $\ldots \ldots \ldots \ldots \ldots \ldots \ldots \ldots \ldots \ldots \ldots \ldots$

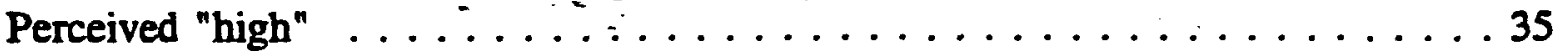

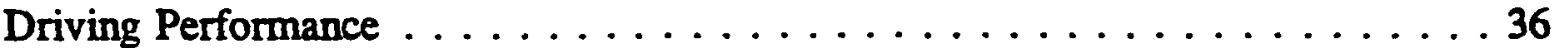

Perceived Driving Quality and Effort $\ldots \ldots \ldots \ldots \ldots \ldots \ldots \ldots \ldots \ldots$

Willingness to Drive . . . . . . . . . . . . . . . . 39

Perceived Alertness, Contentedness and Calmnèss . . . . . . . . . . 39

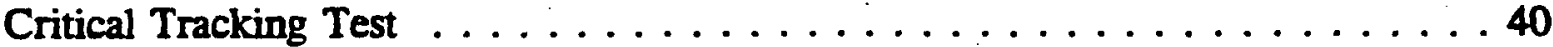

Hand Steadiness Test . . . . . . . . . . . . . . . . 40

Heart Rate and Blood Pressure . . . . . . . . . . . . . . . . . 41

Intra-Subject Relations between Variables . . . . . . . . . . . . . . 42

Inter-Subject Relations between Variables . . . . . . . . . . . . 42

Discussion . . . . . . . . . . . . . . . . . . . . 44

Chapter 5 Marijuana and Driving on a Normal Highway in Traffic . . . . . . . . 49

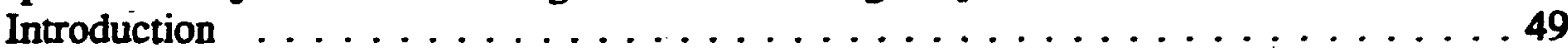

Methods . . . . . . . . . . . . . . . . . . . . 49

Subjects . . . . . . . . . . . . . . . . . . 49

Design, Doses and Administration . . . . . . . . . . . . 50

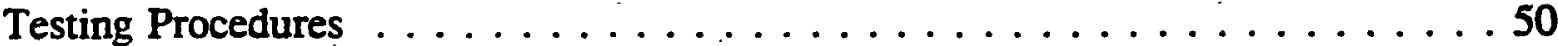

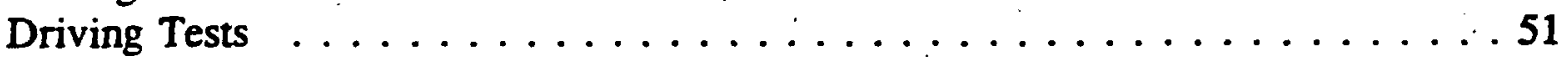

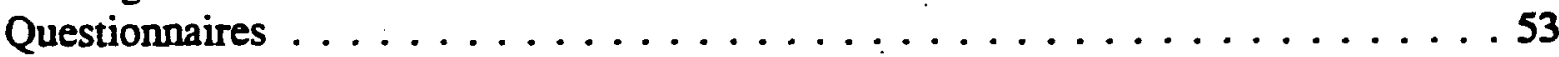

Laboratory Tests . . . . . . . . . . . . . . . . . . . 53

Physiological Assessments . . . . . . . . . . . . . . . 54

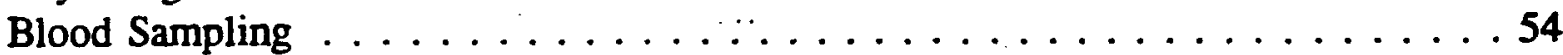

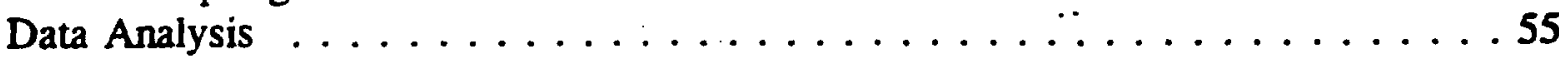

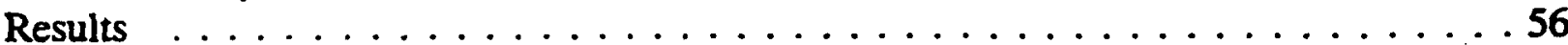

Test-Retest Correlations and Consistency of Performance $\ldots \ldots \ldots \ldots \ldots$

Plasma Concentrations of the Drug $\ldots \ldots \ldots \ldots \ldots \ldots \ldots \ldots \ldots \ldots$.

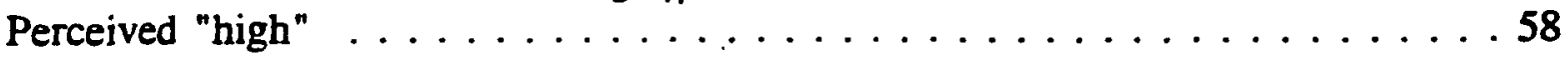

Driving Performance in the Standard Driving Test . . . . . . . . . . . 59

Driving Performance in the Car Following Test $\ldots \ldots \ldots \ldots 61$

Willingness to Drive . . . . . . . . . . . . . . . . 64

Perceived Alertness, Contentedness and Calmness . . . . . . . . . 65 


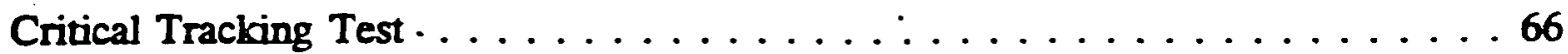

Hand Steadiness Test . . . . . . . . . . . . . . . . . 67

Body Sway . . . . . . . . . . . . . . . . . . .67

Heart Rate . . . . . . . . . . . . . . . . . 68

Intra-Subject Relations between Variables $\ldots \ldots \ldots \ldots \ldots \ldots \ldots$

Inter-Subject Relations between Variables $\ldots \ldots \ldots \ldots \ldots \ldots \ldots \ldots$

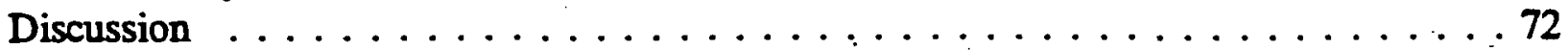

Chapter 6 Marijuana, Alcohol and Urban City Driving $\ldots \ldots \ldots \ldots \ldots 77$

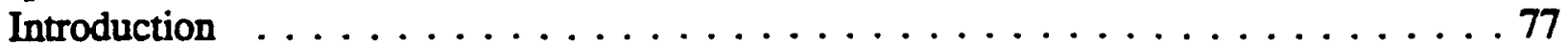

Methods . . . . . . . . . . . . . . . . . . . . 79

Subjects . . . . . . . . . . . . . . . . . 79

Design, Doses and Administration . . . . . . . . . . . . . 79

Testing Procedures . . . . . . . . . . . . . . . . . 80

Driving Test and Scoring Methods $\ldots \ldots \ldots \ldots \ldots \ldots \ldots \ldots \ldots \ldots \ldots$

Questionnaires . . . . . . . . . . . . . . . . . . . 84

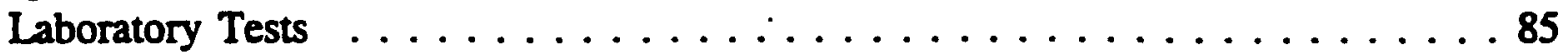

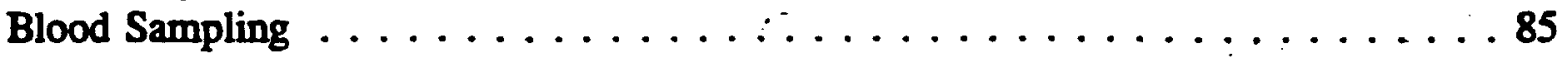

Data Analysis ... . . . . . . . . . . . . . . . 85

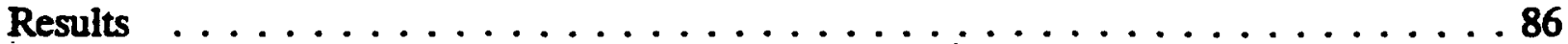

Plasma Concentrations of the Drugs . . . . . . . . . . . 86

Perceived "high" and "drunkness" . . . . . . . . . . . . . . 86

Driving Performance Measured by the Molecular Approach . . . . . . . . 87

Driving Performance Measured by the Molar Approach . . . . . . . . . . 87

Perceived Driving Quality and Effort to Accomplish the Test . . . . . . . . . 89

Willingness to Drive . . . . . . . . . . . . . . . . 90

Perceived Alertness, Contentedness and Calmness . . . . . . . . . . 90

Hand Steadiness Test . . . . . . . . . . . . . . . . . . .90

Time Perception Test . . . . . . . . . . . . . . . . . . . 92

Inter-Subject Relations between Variables $\ldots \ldots \ldots \ldots \ldots \ldots 2$

Discussion . . . . . . . . . . . . . . . . . . .93

Chapter 7 General Discussion, Conclusions and Recommendations .. . . . . . . . . . 97

Introduction $\ldots \ldots \ldots \ldots \ldots \ldots \ldots \ldots \ldots \ldots \ldots \ldots \ldots \ldots \ldots \ldots \ldots$

THC Doses . . . . . . . . . . . . . . . . . . . . . . .98

The Driving Tests . . . . . . . . . . . . . . 100

Effects of THC on Driving Performance . . . . . . . . . . . . 102

Drug Plasma Concentrations and Driving Performance . . . . . . . . 107

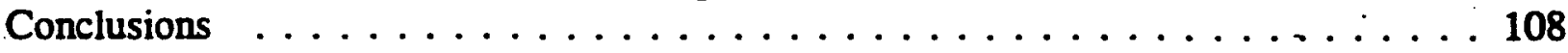

Recommendations for Future Research . . . . . . . . . . . . . . 109

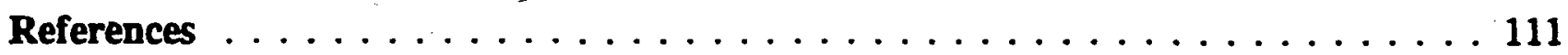

Appendix A. Questionnaires . . . . . . . . . . . . . . . . 117

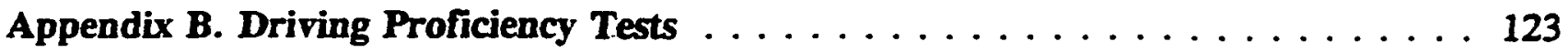




\section{LIST OF ABBREVIATIONS}

11-OH-THC 11-Hydroxy- $\Delta^{9}$-Tetrahydrocannabinol

ANWB Algemene Nederlandse Wielrijders Bond (Royal Dutch Tourist Association)

BAC Blood Alcohol Concentration

CS-C Curve Surface in Body Sway Test with Eyes Closed

CS-O Curve Surface in Body Sway Test with Eyes Open

CTT Critical Tracking Test

CV-H Coefficient of Variation (SD/M) of Headway

CV-IBI Coefficient of Variation (SD/M) of Interbeat Interval Times

DOT

ECG

EtOH

Department of Transportation

Electrocardiogram

IBI

Ethanol

Interbeat Interval Time

$\therefore$ MANOVA Multivariate Analysis of Variance

NHTSA National Highway Traffic Safety Administration

NIDA National Institute on Drug Abuse

PWR-HR Relative Amplitude in the Power Density Spectrum of Heart Rate between the Frequencies of .07 and $.14 \mathrm{~Hz}$

RT Reaction Time

SD Standard Deviation

SDLP Standard Deviation of Lateral Position

SDSP Standard Deviation of Speed

SDST Standard Deviation of Steering Wheel Angle

SE - Standard Error (i.e. SD divided by the Square Root of the Number of Observations)

SED . . Standard Error of the Mean Difference

SP Mean Speed

THC $\Delta^{9}$-Tetrahydrocannabinol

THC-COOH 11-Nor- $\Delta^{9}$-Tetrahydrocannabinol-9-Carboxylic Acid 


\section{ACKNOWLEDGEMENTS}

This research program was sponsored by the Office of Program Development and Evaluation, National Highway Traffic Safety Administration (NHTSA), of the U.S. Department of Transportation (DOT). We are grateful to T.E. Auderson for initiating the program and to J. Frank for sharing the duties of monitoring it. The Road Safety Department of the Dutch Ministry of Transport, Public Works and Watermanagement functioned as an intermediary regarding technical and financial affairs between DOT and the official contractor, the University of Limburg. Furthermore, the alcohol part of the city driving study (Chapter 6) was sponsored by the Dutch Ministry. We thank Drs. J.T. Busstra for monitoring the project locally.

Before the program started an Independent Advisory Committee was formed whose function was to ensure that the program proceeded in accordance with all medical and legal safety. standards. This committee comprised: Mr. J. Oosterhof, Assistant District Attorney and J. Bardoul, Municipal Traffic Attorney, for the City of Maastricht; Dr. H.C. Milius, psychiatrist and member of the University's Medical Ethics Committee; and Drs. J. Tjoeng, Dutch Regional Inśpector for Public Health (Drugs).

Approvals for individual studies were separately obtained from the University's Medical Ethics Committee. A permit for obtaining, storing and administering marijuana was obtained from the Dutch Drug Enforcement Administration.

We are very grateful to'P.G.S.M. Geurts, Chief Traffic Administrator for the Dutch Federal Police, District Limburg, Ing. F.V.J.M. Schepers, Head, Highway Administration South, Department Traffic and Transportation, and Ing. H.P.A. Thegels, Head, Technical Services, Department of Public Works, District Limburg for their cooperation and readiness to close part of a primary highway in Southern Limburg for the first driving study. We thank all those involved in closing and opening the highway every evening during the study.

Plasma samples were analyzed by Prof. Dr. M.R. Möller and his colleagues from the Institute of Legal Medicine from the Department of Forensic Toxicology, University of Homburg, Germany. We thank them very much for their important collaboration.

Medical, technical and administrative support was obtained from our respective colleagues, M. van Boxtel, MD, A. van Houte, MD, Ing. M. Hendrickx, Mrs. V. van der Varst and Mrs. S. Aldenhoff. We are also grateful to all those who assisted the program manager during data collection: B. Bekkers, L. de Beukelaar, Ph. Bouwmeester, A. Casteleyn, J. Dör, R. van Drumpt, A. Gresnigt, M. Heeres, A. van Houte, MD, R. Koppers, C. van Leeuwen, MD, B. Liederkerken, W. Linders, I. Mövig, J. van Oostrum, A. Rademaker and M. Uiterwijk.

Mr. H. Brauers, our licensed driving instructor, was chiefly responsible for safety during driving tests. We deeply respect the care with which he and his colleagues, Mr. and Mrs. Godt, F. Herzberg, and W. Jennissen, ensured the subjects' safety throughout the entire program.

Finally, we gratefully appreciate all of the effort devoted by the subjects, more than seventy in total, during their participation in the study. 


\section{EXECUTIVE SUMMARY}

This report concerns the effects of marijuana smoking on actual driving performance. It presents the results of one pilot and three actual driving studies which were conducted between april 1990 and march 1992. The program was funded by the U.S. National Highway Traffic Safety Administration (NHTSA), with the exception of the alcohol part of the city driving study which was sponsored by the Dutch Road Safety Directorate of the Dutch Ministry of Transport and Public Works. The project was conducted by the Instinute for Drugs, Safety and Behavior of the University of Limburg, Maastricht, The Netherlands. The major objectives of the program were to determine the dose-response relationship between $\Delta^{9}$-tetrahydrocannabinol (THC), marijuana's main constituent, and objectively and subjectively measured aspects of real-world driving; and, to determine whether it is possible to correlate driving performance impairment with plasma concentrations of the drug or a metabolite. A variety of driving tests were employed, including: maintenance of a constant speed and lateral position during uninterrupted highway travel, following a leading car with varying speed on a highway, and city driving. The purpose of applying different tests was to determine whether similar changes in performance under the influence of THC occur in all, thereby indicating a general drug effect on driving ability.

Chapter One provides background information about the drug, its pharmacological properties, the prevalence of its use, and a review of marijuana smoking and traffic safety. THC's effects on the ability of drivers to operate safely in traffic situations have traditionally been determined in two ways: from epidemiological surveys of users' involvement in traffic accidents and from empirical studies to measure the drug's influence on skills related to driving, or driving itself. Epidemiology shows that people drive after marijuana use and that drivers involved in accidents often show the drug's presence. The results are, however, inconclusive because of the high proportion of cases which also involve alcohol use and the lack of proper control groups. Therefore, the extent marijuana contributes to traffic accident causality remains obscure. Results from driving simulator and closed-course tests show that THC in single inhaled doses up to about $250 \mu \mathrm{g} / \mathrm{kg}$ has relatively minor effects on driving performance, certainly less than blood alcohol concentrations (BACs) in the range of 0.08-0.10 $\mathrm{g \%}$.

Chapter Two describes the studies of the program and certain procedures that were common to all. These were subject recruiting, compliance with ethical and legal standards, screening for the presence of other illicit drugs and alcohol, blood sampling procedures and quantitative analyses. Subjects in all studies were recreational users of cannabis, i.e.-smoking marijuana or hashish more than once a month but not daily. They were all healthy, between 21 and 40 years of age, had normal weight and binocular acuity, and were licensed to drive an automobile. Subjects were accompanied in every driving test by an licensed driving instructor, experienced in supervising subjects who operated under the influence of medicinal drugs in previous studies. Redundant control system in the test vehicle was available for controlling the car if emergency sinuations should arise. Marijuana and placebo marijuana cigarettes were supplied by the U.S. National Institute on Drug Abuse (NIDA).

Chapter Three presents the results of the pilot study. It was conducted in a hospital under strict medical supervision to identify THC doses that recreational marijuana users were likely to consume before driving. Twenty-four subjects, twelve males and twelve females, participated. 
They were allowed to smoke part or all of the THC.content in three cigarettes until achieving the desired psychological effect. Cigarettes were smoked through a plastic holder in a manner determined by the subjects. The only requirement was to smoke continuously for a period not exceeding 15 minutes. When subjects voluntarily stopped smoking, cigarettes were carefully extinguished and retained for subsequent gravimetric estimation of THC consumed. Six subjects consumed one cigarette, thirteen smoked two and four smoked three. The average amount of THC consumed was $20.8 \mathrm{mg}$, after adjustment for body weight, $308 \mu \mathrm{g} / \mathrm{kg}$. There was no significant difference between males and females with respect to the weight adjusted preferred dose. It was decided that the maximum dose for subsequent driving studies would be $300 \mu \mathrm{g} / \mathrm{kg}$. This is considerably higher than doses that have usually been administered to subjects in experimental studies (typically, 100-200 $\mu \mathrm{g} / \mathrm{kg}$ THC).

The study provided the opportunity for obtaining valuable information about THC's pharmacokinetics and its pharmacodynamic effects after marijuana smoking. Blood samples were repeatedly taken for measuring plasma concentrations of THC and its major inactive metabolite, THC-COOH. The subjects repeatedly performed certain simple laboratory tests, estimated their levels of intoxication and indicated their willingness to drive under several specified conditions of urgency. Heart rate was measured at these times. The secondary purpose of the pilot study was that of specifying relationships between [THC] and [THC-COOH] with changes in the other physiological, performance or subjective variables. Other results from the pilot study showed that perceived "high" and heart rate are very sensitive measures of marijuana intoxication which confirms prior findings. Impairments in laboratory tests performance were found at the time of peak subjective feelings but generally, objective impaiment dissipated more rapidly than the feelings themselves.

The first driving study, described in Chapter Four, was conducted on a highway closed to other traffic. One objective of the study was to determine whether it would be safe to repeat the study on a normal highway in the presence of other traffic. The second objective was to define the dose-effect relationship between inhaled THC dose and driving performance. The same twelve men and twelve women who participated in the pilot study served again as the subjects. They were treated on separate occasions with THC doses of $0,100,200,300 \mu \mathrm{g} / \mathrm{kg}$. Treatments were administered double-blind and in a counterbalanced order. On each occasion, subjects performed a road tracking test beginning $\mathbf{4 0}$ minutes after initiation of smoking and repeated one hour later. The test, developed and standardized by O'Hanion et al. $(1982,1986)$, involved maintaining a constant speed at $90 \mathrm{~km} / \mathrm{h}(56 \mathrm{mph})$ and a steady lateral position between the delineated boundaries of the traffic lane. Subjects drove $22 \mathrm{~km}$ (13.6 mi) on a primary highway and were accompanied by a licensed driving instructor. The latter was charged with responsibility for ensuring safety at all times and was able to intervene, if necessary, using redundant vehicular controls. The primary dependent variable was the standard deviation of lateral position (SDLP), which has been shown to be both highly reliable and very sensitive to the influence of sedative drugs and alcohol. Other dependent variables were mean speed, and standard deviation of speed and steering wheel angle. Blood samples were taken prior to each driving test; and, performance in critical tracking and hand steadiness tests, heart rate, and blood pressure were measured after its termination. Questionnaires were repeatedly administered to estimate the "high" and other subjective feelings.

All subjects were willing and able to finish the driving tests without great difficulty. The study demonstrated that marijuana impairs driving performance as measured by an increase in SDLP; all three THC doses significantly affected SDLP relative to placebo. The driving 
performance decrement after smoking marijuana persisted almost undiminished for two hours after smoking while drug plasma concentrations, perceived "high" and heart rate elevation had decreased. Marijuana's effects on SDLP were compared to those of alcohol obtained in a very similar study by Louwerens et al. $(1985,1987)$. It appeared that THC's effects on SDLP were equivalent to those associated with BACs in the range of $0.03-0.07 \mathrm{~g} \%$. Other driving performance measures were not significantly affected by THC. Intersubject correlations between plasma concentrations of the drug and driving performance after every dose were essentially nil. Thus, driving impairment cannot be predicted by prévailing plasma concentrations of THC or THC-COOH. Driving impairment was also not related to performance in the laboratory tests. Both the observed degree of driving impairment, and what subjects saidsand did, indicated that normal safeguards would be sufficient for ensuring safety in further testing. Hence, the final conclusion was to repeat this study on a normal highway in the presence of other traffic.

The second driving study, described in Chapter Five, was conducted to come a step closer to driving reality than its predecessor. Driving tests were now conducted on a highway in the presence of other traffic. The major objective of this study was to confirm the relationship between inhaled THC dose and lateral position variability in the context of a standard road tracking test. A secondary objective was to measure performance in another actual driving test, i.e. car following. The third objective was to continue efforts to correlate plasma concentrations of THC and THC-COOH with driving performance impairment as measured in both tests.

A new group of sixteen subjects, equally comprised of men and women, participated in this study. A conservative approach was chosen in designing the present study in order to satisfy the strictest safety requirements. That is, the study was conducted according to an ascending dose series design where both active drug and placebo conditions were administered, double-blind, at each of three THC dose levels. THC doses were the same as those used in the previous study, namely 100,200 , and $300 \mu \mathrm{g} / \mathrm{kg}$. Cigarettes appeared identical at each level of treatment conditions and were smoked through a plastic holder in a fashion determined by the subject within a time limit of 10 minutes. If any subject would have reacted in an unacceptable manner to a lower dose, he/she would not have been permitted to receive a higher dose.

Two subjects at a time commenced smoking. Thirty minutes after onset of smoking the subjects performed a battery of laboratory tests (tracking, hand steadiness and body sway), yielded a blood sample, and rated their "high" and other subjective feelings. They were then transported to a primary highway were the driving tests were performed. Two instrumented vehicles were employed. The subjects performed the car following test on a $16 \mathrm{~km}(9.9 \mathrm{mi})$ segment of the highway for about twelve minutes. After conclusion of the car following test, both subjects then commenced the road tracking test in separate instrumented vehicles. The highway was the same as for the car following test. Subjects drove $64 \mathrm{~km}(40 \mathrm{mi})$ without stopping in about 50 minutes. At the conclusion of this test, both subjects participated again in the car following test. Subjects were then transported back to the laboratory where they rated subjective feelings, yielded a blood sample, and repeated the test battery. The subjects' heart rate was registered continuously during both driving tests.

The road tracking test was the same as in the previous study except for its duration and the presence of other traffic. Subjects were instructed to maintain a constant speed of $95 \mathrm{~km} / \mathrm{h}$ $(59 \mathrm{mph})$ and a steady lateral position between lane boundaries in the right traffic lane. They were allowed to deviate from this only if it would become necessary to pass a slower vehicle in the same lane. Data from the standard test were analyzed to yield the same performance measures as in the previous study; i.e. SDLP, mean and standard deviation of speed, and 
standard deviation of steering wheel angle. The car following test measures drivers' ability to perceive changes in a preceding vehicle's speed and to react in a manner maintaining a constant headway. It began as the preceding and the following vehicle, respectively driven by one of the driving instructors and the subject, operated in tandem on the slower traffic lane while travelling at a speed of $100 \mathrm{~km} / \mathrm{h}(62 \mathrm{mph})$. The subject was instructed to maintain a $50 \mathrm{~m}$ (164 ft) beadway however the preceding vehicle's speed might vary. After driving in this manner for about one minute, the operator of the preceding vehicle released the accelerator pedal allowing its speed to fall to $80 \mathrm{~km} / \mathrm{h}(50 \mathrm{mph})$. Immediately thereafter, the operator of the preceding vehicle accelerated to $100 \mathrm{~km} / \mathrm{h}(62 \mathrm{mph})$. The duration of one deceleration and acceleration maneuver was approximately 50 seconds and six to eight, depending upon traffic density, were executed during one test. The subject's average reaction time to the movements of the leading vehicle, mean headway and coefficient of variation of headway during maneuvers were taken as the dependent variables from this.

All subjects were able to complete the series without suffering any untoward reaction while driving. Road tracking performance in the standard test was impaired in a dose-related manner by THC and confirmed the results obtained in the previous closed highway study. The $100 \mu \mathrm{g} / \mathrm{kg}$ dose produced a slight elevation in mean SDLP, albeit nearly significant. The $200 \mu \mathrm{g} / \mathrm{kg}$ dose produced a significant elevation, of dubious practical relevance. The $300 \mu \mathrm{g} / \mathrm{kg}$ dose produced a highly significant elevation which may be viewed as practically relevant but unexceptional in comparison with similarly measured effects of many medicinal drugs. Following marijuana smoking subjects drove with an average speed that was only slightly lower than after placebo and very close to the prescribed level.

In the car following test, subjects maintained a headway of 45-50 m (148-164 ft). while driving in the successive placebo conditions. They lengthened mean headway by 8,6 and $2 \mathrm{~m}$ $(26.2,19.7$ and $6.6 \mathrm{ft}$ ) in the corresponding THC conditions after 100,200 and $300 \mu \mathrm{g} / \mathrm{kg}$, respectively. The initially large drug-placebo difference and its subsequent decline is a surprising result. Our explanation for this observation is that the subjects' caution was greatest the first time they undertook the test under the influence of THC and progressively less thereafter. Reaction time to changes in the preceding vehicle's speed increased following THC treatment, relative to placebo. The administered THC dose was inversely related to the change in reaction time, as it was to headway. However, increased reaction times were partly due to longer headway. Statistical adjustment for this confounding resulted in smaller and non-significant increases in reaction time following marijuana treatment, the greatest impairment $(0.32 \mathrm{~s})$ being observed in the first test following the lowest THC dose. Headway variability followed a similar pattern as mean headway and reaction time; the greatest impairment was found following the lowest dose.

An important practical objective of this study was to determine whether degrees of driving impairment can be accurately predicted from either measured concentrations of THC in plasma or performance measured in potential roadside "sobriety" tests of tracking ability or hand and posture stability. The results, like many reported before, indicate that none of these measures accurately predicts changes in actual driving performance under the influence of THC.

The program then proceeded into the third driving study, presented in Chapter Six, which involved tests conducted in high-density urban traffic. There were logical and safety reasons for restricting the THC dose to $100 \mu \mathrm{g} / \mathrm{kg}$. It was given to a group of regular cannabis users, along with placebo. For comparative purposes another group of regular alcohol users were treated with a modest dose of their preferred recreational drug, and again placebo, before undertaking the 
same city driving test. Two groups of sixteen new subjects apiece, equally comprised of men and women, participated. Subjects in the alcohol group were regular users of alcohol but not marijuana. Both groups were treated on separate occasions with active drug and placebo. Active marijuana was administered to deliver $100 \mu \mathrm{g} / \mathrm{kg}$ THC. The driving test commenced 30 minutes after smoking. The alcohol dose was chosen to yield a BAC approaching $0.05 \mathrm{~g} \%$ when the driving test commenced 45 minutes after onset of drinking. Active drug and placebo conditions were administered double-blind and in a counterbalanced order in each group.

Driving tests were conducted in daylight over a constant $17.5 \mathrm{~km}(10.9 \mathrm{mi})$ route within the city limits of Maastricht. Subjects drove their placebo and active drug rides through heavy, medium and low density traffic on the same day of the week, and at the same time of day. Two scoring methods were employed in the present study. The first, "molar" approach, required the driving instructor acting as the safety controller during the tests to retrospectively rate the driver's performance using a standard scale. The second, a more "molecular" approach, involved the employment of a specially trained observer who applied simple and strict criteria for recording when the driver made or failed to make each in a series of observable responses at predetermined points along a chosen route. Immediately prior to and following the driving tests subjects performed hand steadiness and time perception tests, yielded a blood sample, and were administered the same subjective questionnaires used in the previous studies.

The study showed that a modest dose of alcohol $(\mathrm{BAC}=0.04 \mathrm{~g} \%$ ) produced a significant impairment in city driving as measured by the molar approach, relative to placebo. More specifically, alcohol impaired vehicle handling and traffic maneuvers. Marijuana, administered in a dose of $100 \mu \mathrm{g} / \mathrm{kg}$. THC, on the other hand, did not significantly change mean driving performance as measured by this approach. Neither alcohol nor marijuana significantly affected driving performance measures obtained by the molecular approach indicating that it may be relatively insensitive to drug-induced changes.

Driving quality as rated by the subjects contrasted with observer ratings. Alcohol impaired driving performance according to the driving instructor but subjects did not perceive it; marijuana did not impair driving performance but the subjects themselves perceived their driving performance as such. Both groups reported about the same amount of effort in accomplishing the driving test following placebo. Yet only subjects in the marijuana group reported significantly higher levels of invested effort following the active drug. Thus, there was evidence that subjects in the marijuana group were not only aware of their intoxicated condition but were also attempting to compensate for it. These seem to be important findings. They support both the common belief that drivers become overconfident after drinking alcohol and investigators' suspicions that they become more cautious and self-critical after consuming low THC doses by smoking marijuana.

The laboratory performance tests also discriminated between the drugs' effects. Hand steadiness was impaired following THC and improved following alcohol, relative to placebo. The difference between the drugs' effects was significant, both before and after the driving test. Impairment after THC was about as much as that produced by the same dose in the previous study, indicating equivalent sensitivities of the present and previous groups. Production of time intervals was not affected by alcohol, but THC significantly shortened interval production, relative to placebo.

Drug plasma concentrations were neither related to absolute driving performance scores nor to the changes that occurred from placebo to drug conditions. With respect to THC, these results 
confirm the findings in previous studies. They are somewhat surprising for alcohol but may be due to the restricted range of ethanol concentrations in the plasma of different subjects.

Chapter Seven concludes the report with a general discussion of the results of the program and ends with a list of conclusions and recommendations. It starts with a discussion of the THC dose which marijuana users actually prefer for achieving their desired "high". Several questions are raised and discussed, such as: how do people regulate their THC consumption, what role plays familiarization with the drug, and what would the preferred dose have been if marijuana of much higher potency were smoked. The discussion then continues with a description of the differences between the driving tests in terms of the type of information processing each requires, automatic $v s$ controlled, and the relevance of each to traffic safety.

Attention is further focussed on the effects of THC on driving performance. The results of the studies corroborate those of previous driving simulator and closed-course tests by indicating that THC in single inhaled doses up to $300 \mu \mathrm{g} / \mathrm{kg}$ has significant, yet not dramatic, dose-related impairing effects on driving performance. Standard deviation of lateral position in the road tracking test was the most sensitive measure for revealing THC's adverse effects. This is because road tracking is primarily controlled by an automatic information processing system which operates outside of conscious control. The process is relatively impervious to environmental changes but highly vulnerable to internal factors that retard the flow of information through the system. THC and many other drugs are among these factors. When they interfere with the process that restricts SDLP, there is little the afflicted individual can do by way of compensation to restore the situation. Car following and, to a greater extent, city driving performance depend more on controlled information processing and are therefore more accessible for compensatory mechanisms that reduce the decrements or abolish them entirely.

It appears that performance is more affected by THC in laboratory than actual driving tests. Several reasons that may account for the apparent discrepancy are discussed. First, laboratory tests are experimentally controlled by drastic simplification which may affect a subject's motivation to perform the test by making it appear "unreal". Secondly, the restriction of response options in laboratory performance tests leave fewer possibilities for compensation. In real life, drivers always apply numerous skills in parallel and series. Should one become deficient, they are often able to compensate in a number of ways to achieve a satisfactory level of proficiency. Finally, after learning to drive, subjects possess such skills in abundance and one can only demonstrate how they vary with drug effects in the real task or a very close approximation thereof. Profound drug impairment constituting an obvious traffic safety hazard could as easily be demonstrated in a laboratory performance test as anywhere else. But THC is not a profoundly impairing drug. It does affect automatic information processing, even after low doses, but not to any great extent after high doses. It apparently affects controlled information processing in a variety of laboratory tests, but not to the extent which is beyond the individual's ability to control when he is motivated and permitted to do so in real driving.

Marijuana's effects on driving performance were compared to those of many other drugs. It was concluded that THC's effects after doses up to $300 \mu \mathrm{g} / \mathrm{kg}$ never exceed alcohol's at BACs of $0.08 \mathrm{~g} \%$; and, were in no way unusual compared to many medicinal drugs'. Yet THC's effects differ qualitatively from many other drugs, especially alcohol. Evidence from the present and previous studies strongly suggests that alcohol encourages risky driving whereas THC encourages greater caution, at least in experiments. Another way THC seems to differ qualitatively from many other drugs is that the former's users seem better able to compensate for its adverse effects while driving under the influence. Still one can easily imagine situations 
where the influence of marijuana smoking might have an exceedingly dangerous effect; i.e., emergency situations which put high demands on the driver's information processing capacity, prolonged monotonous driving, and after THC has been taken with other drugs, especially alcohol.

Finally, the relation between driving impairment following marijuana smoking and plasma concentrations of THC and THC-COOH is discussed. It appears not possible to conclude anything about a driver's impairment on the basis of his/her plasma concentrations of THC and THC-COOH determined in a single sample.

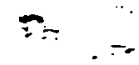


Marijuana's effects on the ability of drivers to operate safely in traffic situations have traditionally been determined in two ways: from epidemiological surveys of users' involvement in traffic accidents and from empirical studies to measure the drug's influence on skills related to driving and driving itself. A review of results obtained from both approaches, partly relying upon previously published reviews, is provided. Its purpose is providing the reader a broader context in which the present report should be viewed. First, however, the drug and its pharmacological properties, and the prevalence of its use, will be discussed.

\section{THE DRUG AND ITS PHARMACOLOGICAL PROPERTIES}

Marijuana is the common name for any part of the hemp plant, cannabis sativa, or extracts which possess characteristic psychoactive properties in man. The plant contains more than $\mathbf{4 0 0}$ compounds. More than 60 , the cannabinoids, are :specific to that plant. The majority of the cannabinoid products are pharmacologically inactive. The major active product and that primarily responsible for the physiological and psychological effects of marijuana smoking is $\Delta^{9}$-tetrahydrocannabinol (THC).

- Besides being used as an intoxicant to produce a psychological "high" or euphoria, marijuana has been used as a sedative and analgesic (Maykut, 1985) but also, in Eastern countries, for relieving fatigue and stimulating appetite (Murray, 1985). It reliably produces both tachycardia and marked conjunctivitis. The mechanisms by which THC produces these physiological effects or the psychological effects sought by its users are still poorly understood (Jaffe, 1990). As a therapeutic agent, marijuana has been successfully applied in some cases of glaucoma (Relman, 1982) and anorexia nervosa (Zinberg, 1979). The most promising potential clinical use of marijuana is in the treatment of nausea and vomiting caused by cancer chemotherapy (Relman, 1982; Vinciguerra et al., 1988; Randall, 1990). Synthetically formulated THC was approved in 1985 by the U.S. Food and Drug. Administration (FDA) for use in the treatment of emesis, and is now marketed under the trade name Marinol (Unimed, Somerville, NJ). Patients suffering from AIDS may also benefit from marijuana because of its antiemetic and appetite stimulating effects. Yet there are also reports of adverse health effects of marijuana smoking on fetal growth, sperm cell motility, female reproductive hormone function, immunological system, cardiopulmonary system and central nervous system (Jaffe, 1990; Mendelson; 1987; Maykut, 1985).

The THC content in marijuana cigarettes varies in the United States from about $0.5 \%$ to $11 \%$ (Jaffe, 1990). In The Netherlands, seized hemp material usually contains about 10\% THC, though it may range from 5 to $15 \%$ and, in exceptional cases, to $25 \%$ (Dutch Forensic Laboratory, personal communication). The inhaled dose in marijuana smoke varies also widely, depending upon the smoking technique and the amount altered by pyrolysis. In general it is thought that no more than about $25 \%$ of the available THC enters the circulation when marijuana is smoked in the usual manner (Davis et al., 1984; Ohisson et al., 1980; Agurell and Leander, 
1971), though if it were possible to continuously inhale smoke from an entire cigarette, up to 70\% would become available (Agurell and Hollister, 1986).

The metabolism of THC is exceedingly complex and more than 80 metabolites are known to be formed in man. After marijuana smoking or THC injection, the first metabolite, 11hydroxy-THC (11-OH-THC) is formed in the lungs and liver. Its peak concentration in relation to the parent compound's is about 1:10-20 (Wall et al., 1983). After oral THC the ratio is about $1: 1-2$. Because this metabolite's psychological activity is equipotent to the parent's, it contributes to the total marijuana effect, particularly when the drug is ingested. 11-OH-THC is converted by the liver into a number of inactive metabolites. The primary pathway leads to the formulation of 11-nor-THC-9-carboxylic acid (THC-COOH), the most abundant inactive metabolite in plasma, and in urine where it is partially conjugated.

Plasma concentrations of THC peak during the smoking process and decline in sequential exponential phases; a redistribution ( $\alpha$ ) phase wherein the drug passes rapidly out of the plasma and into fatty tissues including the brain, followed by a much more prolonged elimination (B) phase wherein it is metabolized and excreted in urine and feces. The $\alpha$-phase half life $\left(t_{1 / \alpha \alpha}\right)$ is only about 30 minutes, whereas that of the B-phase $\left(t_{1 / B}\right)$ varies between 18 and 36 hours depending upon the individual (Wall et al., 1983; Chiang and Barnett, 1984; Agurell et al., 1986).

The peak plasma concentration of 11-OH-THC is achieved within 15-30 minutes and from there declines according to essentially the same pharmacokinetic profile as its parent. The 'rise in THC-COOH's plasma concentration is relatively slow, reaching an ill defined peak in differênt individuals within 1-2 hours. Its elimination follows a monoexponential profile with various individuals showing $t_{1 / B}$ 's from less then 24 to more than 72 hours. It is interesting to note that the inactive metabolite's mean plasma concentration exceeds that of THC from about the first hour onward.

Though peak concentrations of THC are achieved during smoking, the maximum psychological effect (the "high") occurs 15-30 minutes after its cessation, suggesting that brain concentrations increase as plasma concentrations decrease. Both peak concentrations and maximum "high" are roughly proportional to the inhaled THC dose, but correlations between these parameters measured simultaneously at times 3-240 min after the cessation of smoking are, albeit significant, not especially strong. For example, Ohlsson et al. (1980) found the overall correlation for repeated measurements obtained from 11 experienced smokers to be $r=0.53$. After four hours the psychological "high" had vanished and plasma. THC levels were very low. Great interindividual variation exists in plasma levels of THC after smoking and this variation cannot be attributed to the regularity of marijuana use (Lindgren et al., 1981; Agurell and Hollister, 1986).

Available evidence leads to the conclusion that it is usually impossible to predict the psychological effects of THC from its determination in a single plasma sample. But this is not the same as saying that no biological index of marijuana intoxication will ever be found. One possible candidate is THC's inactive metabolite THC-COOH. The relationship between this metabolite's plasma concentration and the perceived "high" after marijuana smoking has never been defined, although both parameters were measured in the study by Perez-Reyes et al. (1982). Peak and time integrated THC-COOH concentrations were proportional to the ;administered THC doses. Interestingly, the occurrence of the peak THC-COOH concentration coincided in time with the subjects' report of maximum "high". The authors failed, however, to measure, or at least report, the correlation between plasma THC-COOH concentration and 
subjective feelings because of the metabolite's pharmacological inactivity. Yet this coincidence might signify a useful epiphenomenal correlation. This possibility was repeatedly explored in this program.

\section{PREVALENCE OF MARIJUANA USE}

Marijuana usage prevalence peaked in the late 1970 s and has been declining ever since. Still, marijuana is by far the most commonly used illicit drug in the United States (Jessor et al., 1986; Johnston et al., 1992). The most recent data äbout marijuana usage prevalence are available from the $17^{\text {th }}$ National Survey of American High School Seniors, and the $12^{\text {th }}$ National Survey of American College Students (Johnston et al., 1992). Life time prevalence among high school seniors declined from $60 \%$ in 1980 to $37 \%$ in 1991 ; and, among college students, from $65 \%$. in 1980 to $46 \%$ in 1991 . Thirty-day prevalence declined in both groups from $34 \%$ in 1980 to $14 \%$ in 1991 . About $9 \%$ of high school seniors and $7 \%$ of college students admitted daily use of marijuana in 1980 . Only $2 \%$ of both groups did in 1991.

A 1988/1989 survey of cannabis use among Dutch students, 10-20 years old, provided different results (Plomp et al., 1990): Considering only those in an age bracket comparable to American high school seniors and recent graduates $(17-20$ yrs; $N=1806)$, the lifetime use prevalence of cannabis was $21 \%$ and $13 \%$ among males and females, respectively. Those who smoked at least once during the last month comprised $5.8 \%$ and $3.0 \%$ of the respective samples. Compared to results obtained from a similar survey executed in 1984 (Van der Wal, 1985) the lifetime and current prevalence of use have risen in The Netherlands by factors of 1.7 and 1.5, respectively.

In short, relatively more young Americans formerly used and still use cannabis than their Dutch counterparts, but the disparities between lifetime and current use prevalences are narrowing due to opposite trends in the two countries.

It is perhaps important to note in this context that possession of cannabis is prohibited by law in The Netherlands, as in the United States. The seriousness of the offense is, however, determined by the amount found in the Dutch user's possession and prosecution is unlikely to occur when that is less than $30 \mathrm{~g}(1 \mathrm{oz})$.

\section{MARIJUANA AND TRAFFIC SAFETY}

\section{Epidemiological Research}

Simpson (1986) has reviewed recent epidemiological evidence regarding marijuana's role in traffic accident causality. His first concern was to determine the frequency of driving after marijuana use in order to identify the proportion of the total driving population who may be considered "at risk" of causing an accident for that reason. His information was derived from two sources: questionnaire surveys of adolescents (16-19 years), who were licensed to drive, and roadside surveys of recent usage among passing motorists.

He mentioned the reasonably consistent results of four questionnaire surveys conducted in the United States or Canada between 1979 and 1982. About one in six teenage drivers admitted driving while smoking or shortly afterward, and about $10 \%$ said they had done so between one and five times during the preceding month. Taken at face value, these results indicate that most 
users do not drive during or shortly after marijuana smoking which implies they do perceive risks associated with marijuana use and driving. Unfortunately it is not easy to generalize these results to older drivers in North America, nor to Europeans who are generally not licensed to drive until older than 18 years of age.

Only two surveys of recent marijuana use among drivers stopped at roadside check points have apparently been reported. These were widely separated in place and time. The first was completed in Canada in 1974 (Smith et al., 1975), the second in Italy in 1982 (Ferrara and Rozza, 1985). Moreover, the former relied upon the drivers' admission of use and the latter upon detection of cannabinoids in urine samples. Nonetheless, the indications of recent marijuana use given by the two sets of results were not grossly different; $4 \%$ by the first and $1.2 \%$ by the second.

The incidence of drivers whose recent use of marijuana resulted in their injury or death in motor vehicle accidents can only be estimated from the detection of THC. in plasma samples obtained shortly after the occurrence of the event. Hemolyzed blood samples obtained from dead victims provide unreliable estimates and analyses of urine samples to determine metabolite concentrations yield no indication of whether the drug was active at the time of the accident.

Terhune (1982) tested 497 injured drivers for the presence of a wide range of drugs during treatment at the Rochester General Hospital in New York. THC in blood was detected in $9.5 \%$ of the drivers, but more than half of them also tested positively for alcohol. Chesher and Starmer (1983) found THC in 6.7\% of 104 injured drivers in New South Wales, Australia, but again about half of them showed alcohol as well. Daldrup et al. (1987) examined 597 blood samples from injured drivers in the region around the German city Düsseldorf for the presence of alcohol. Blood samples having alcohol concentrations (BAC) below $0.13 \mathrm{~g} \%$ were additionally analyzed for the presence of cannabinoids; twenty-five of the 220 blood samples (10\%) were positive. More recently, Soderstrom et al. (1988) determined prior marijuana and alcohol use in 1023 patients who were injured as the result of vehicular and nonvehicular accidents and treated in the Shock Trauma Center in Baltimore, Maryland. THC was found in blood by radioimmunoassay in $34.7 \%$ of the patients, alcohol in $33.5 \%$. Among automobile drivers, the numbers were $31.7 \%$ and $34.6 \%$, respectively. Again, in about $50 \%$ of the marijuana positive cases alcohol was also found. It is not clear why these results contrast with those of previous studies. The most plausible explanation is that residents of the Baltimore area tend, in general, to use THC more often than those in the other regions surveyed.

Canadian and American surveys of fatally injured drivers have generally found the incidence of those showing THC in plasma to be between $3 \%$ and $11 \%$ and in all cases the coincidence of this drug and alcohol was above 70\% (Cimbura et al., 1980, 1982, 1990;. Donelson et al., 1985; Owens, 1981; Mason and McBay, 1984). Disparate results were obtained by.Williams et al. (1985) who found THC in plasma from $37 \%$ of 440 dead drivers in California (80\% in combination with alcohol). The reason for this disparity could be due to the greater prevalence of marijuana use in California but it was more probably related to these investigators' selection criteria: they only included male drivers younger than 35 years of age in their sample. Simpson estimated that if female and older male fatalities had been included in this survey, the overall percentage showing THC would have been about $20 \%$. This figure is still double the estimates from other studies, which reinforces suspicions about the prevalence of marijuana use in California. However neither this nor any other survey allowed for the local and contemporary comparison of the fatally injured percentage of drivers showing THC plasma levels with that of the driving population in general. 
If it is true that the population at risk from driving after marijuana smoking is $4 \%$, or less, then the higher percentages of drivers injured or killed in traffic accidents while showing plasma THC concentrations seem to be an over-representation. However, there are obvious reasons to doubt whether valid estimates of the population at risk in urban North America can be derived from data that are more than 14 years old or were obtained at two locations in Northern Italy. Even if the population at risk is as small as estimated, the surveys of THC incidence in injured or killed drivers have not provided evidence for a causal role of the drug per se in accidents. Alcohol was present in the vast majority of victims showing plasma concentrations of THC. The fact that the two drugs in combination possess a greater risk potential than either alone, is most likely. But the independent contribution of THC to traffic accident causality, particularly in concentrations which are likely to be found in most users who drive, is still questionable.

One major problem in epidemiological research on the relationship between marijuana and traffic accidents is the lack of sound control groups as have been used in studies of alcohol involvement in accidents (e.g. Borkenstein et al., 1964). In those studies BACs derived from breath samples of drivers involved in accidents were compared with those of randomly selected drivers passing the accident site in the same direction at the same time of day and day of week. As Moskowitz (1985) noted, these kind of studies rely on two assumptions that do not hold well for investigations on marijuana. The first is that nearly all drivers will cooperate, which holds for alcohol studies in which typically $97 \%$ are willing to supply a breath sample, but does not hold for marijuana studies in which only $50-75 \%$ are willing to cooperate due to the necessity of sampling blood rather than breath. A second assumption is that drug concentrations found are well correlated with performance impairment, which holds for alcohol but seems not to for marijuana.

One way to circumvent this problem is the use of a culpability index, which reflects the percentage of drivers with detectable drug levels and deemed culpable compared to drug free drivers from the same sample who were also responsible for causing an accident. Warren et al. (1981) reanalyzed the data from Cimbura et al. (1980) and reported that $52 \%$ of the drug free fatally injured drivers were deemed culpable compared to $90 \%$ of those with evidence of marijuana use resulting in a culpability index of 1.7, a level also found for alcohol. Results of two other studies (Terhune, 1982; Donelson et al., 1985) are consistent with these findings, whereas Mason and McBay (1984) found no evidence of marijuana as a risk factor. In contrast to these studies, Williams et al. (1985) found that drivers in whom only marijuana was detected were less likely to be culpable ( $53 \%$ vs $71 \%$ ). In contrast, dead drivers showing only alcohol were judged responsible in $92 \%$ of all cases. Those showing both THC and alcohol were slightly more often responsible for causing the accident than those in whom only alcohol was found. It should be noted, however, that the frequencies of injured drivers showing THC alone are commonly very low and prohibit any definite conclusion.

In summary, epidemiological literature shows that people do drive after marijuana use and that drivers involved in accidents often show the drug's presence, but results are inconclusive especially because of the high proportion of cases that also involve alcohol use. Therefore, the extent marijuana contributes to traffic accident causality remains obscure.

\section{Marijuana's Effects upon Driving Simulator Performance}

Early studies by Crancer et al. (1969), Rafaeisen et al. (1973), Ellingstad et al. (1973) and Moskowitz et al. (1976) utilized the filmed ride approach where subjects had little or no control 
over the presented imagery. Dott (1972) used a different approach for measuring subjects' decisions to pass a preceding car, or not, in the presence of an opposing vehicle portrayed as models on a continuous belt. Doses of inhaled THC varied from about 3 to $22.5 \mathrm{mg}$ $(43-321 \mu \mathrm{g} / \mathrm{kg}$ for $70 \mathrm{~kg}$, or $154 \mathrm{lb}$, persons). Smiley (1986) reviewed these early studies to conclude that THC had (1) not affected vehicle control, (2) increased decision latency before starting, stopping or overtaking, (3) reduced the willingness to accept a risk during passing maneuvers, and (4) impaired speedometer monitoring. Except in the case of one individual who, after inhaling $12 \mathrm{mg}$ THC, repeatedly drove through stop lights during a filmed ride, no particular sign of dangerous driving behavior was observed.

Smiley et al. (1981) conducted the first study using an interactive simulator with accurate visual imagery, though not moving base dynamics. The simulated tasks contained in a 45 -minute scenario included curve following, reacting to wind gusts, car following, route selection from signs, avoiding an obstacle which appeared in front of the simulated vehicle and passing. A visual choice reaction time was also superimposed on driving. Three groups of marijuana users smoked cigarettes containing 0,100 and $200 \mu \mathrm{g} / \mathrm{kg}$ THC on two occasions per dose, once with and once without alcohol. The quantity of alcohol consumed varied between groups to reach intended blood concentrations of $0.00,0.05$ and $0.08 \mathrm{~g} \%$, respectively. To ensure high motivation, good driving was rewarded and blatant errors, such as crashes, were penalized financially. The test began 15 minutes after the cessation of smoking. Both THC doses increased lateral position variability and the highest dose increased speed variability during curve following. Both increased headway variability, and the highest, lateral position variability during car following. Both doses caused the subjects to miss more signs indicating the need to follow another route. The high dose caused the subjects to hit the roadway obstacle more offeten than placebo, and also, to react slower to the subsidiary task. Yet both THC doses caused the subjects to drive in a more conservative manner. They maintained a longer headway while car following, refused more opportunities to pass, and when they did, began this maneuver at a greater distance from the approaching vehicle. Alcohol's effects in this study were generally less than THC's. Chesher (1986) was puzzled by this, calling the alcohol effect "surprisingly small" and its interactive effect with THC, "unclear". Certainly it is so that BACs.of $0.08 \mathrm{~g} \%$ and below have been enough to substantially degrade drivers' control of vehicular lateral position in real driving tests (Louwerens et al., 1987; Ramaekers et al., 1992a).

Stein et al. (1983) conducted two studies of alcohol and marijuana effects using a driving simulator and a 15-minute test scenario that were very similar to those employed by Smiley $e t$ al: (1981). The former administered the two drugs in complete crossover designs. THC doses of 0,50 and $100 \mu \mathrm{g} / \mathrm{kg}$ THC were combined with BACs of 0.00 and $0.10 \mathrm{~g} \%$ in the first study. The same BACs were combined with 100 and $200 \mu \mathrm{g} / \mathrm{kg}$ in the second. This time alcohol had the expected adverse effect on practically-every performance parameter, THC had little effect in the first study and little in the second in spite of the higher dose. The latter did cause the subjects to operate at generally lower speeds, however. The combination of drugs produced widely different individual reactions. After the highest THC dose, the combination produced more adverse reactions than alcohol alone.

\section{Marijuana's Effects upon Actual Driving Performance}

A number of studies on marijuana's effects upon actual car driving have been reported since 1974. All studies but one were carried out on courses closed to other traffic. Klonoff (1974) 
conducted the exceptional study wherein 64 subjects drove on a closed course and 38 also participated in a city driving test. In his first study subjects were assigned to one of three groups that were treated with (1) placebo, (2) $4.9 \mathrm{mg} \mathrm{THC}$, and (3) $8.4 \mathrm{mg}$ THC. They undertook eight tests: a slalom, two tunnel tests, a funnel test, a backing up, turning in a corner, a risk judgment test and an emergency braking test. Except for the latter two, the performance measure was number of cones hit. Subjects performed 20 trials in four blocks of five. Treatments were administered between the third and fourth block and each subject's performance was related to his/her performance predicted by means of regression analysis over the first three blocks. Performance after placebo was as predicted, but after marijuana, significantly worse, though not much. The low dose impaired performance in two tests (tunnel and corner) and the high dose in five (slalom, both tunnel tests, funnel and risk judgment).

Subjects in the city driving test were divided among four groups who were treated with placebo and marijuana, on separate occasions a week apart. The respective groups' treatments were (1) placebo followed by $4.9 \mathrm{mg} \mathrm{THC}$, (2) the same in reverse order, (3) placebo followed by $8.4 \mathrm{mg} \mathrm{THC}$, and (4) the same in reverse order. After smoking a placebo or marijuana cigarette, the subjects drove for 45 minutes over a $16.8 \mathrm{mi}(27.0 \mathrm{~km})$ route on city streets while aspects of their performance were rated by a professional examiner using an abbreviated version of the British Columbia Department of Motor Vehicles' standard driver's licensing test:. All subjects were allowed to complete the test which indicates that their performance never became dangerously unsafe under the drug's influence. Nonetheless, the examiner rated the subjects' performance as significantly worse on scales of judgement and concentration following the highest but not the lowest dose. The majority showed some impairment, but $32 \%$ after the low dose and $16 \%$ after the high dose performed significantly better than they had following placebo suggesting qualitative differences between the drug's effects in different subjects.

Hansteen et al. (1976) tested sixteen subjects in four conditions, (1) placebo alcohol + placebo marijuana, (2) placebo alcohol + marijuana (IHC dose of $21 \mu \mathrm{g} / \mathrm{kg}$ ), (3) placebo alcohol + marijuana (THC dose of $88 \mu \mathrm{g} / \mathrm{kg}$ ), and (4) alcohol (BAC $0.07 \mathrm{~g} \%$ ) + placebo marijuana. Subjects were instructed to drive through a $1.1 \mathrm{mi}(0.7 \mathrm{~km})$ course delineated by traffic cones as quickly as possible but without exceeding $30 \mathrm{mph}(19 \mathrm{~km} / \mathrm{h})$. Performance was measured shortly after smoking and three hours later. Number of cones hit, "rough handling" (superfluous and/or awkward movements as observed by an accompanying investigator), and driving time were scored. More cones were hit and more time was taken to complete each hap after consuming the higher THC dose, but no increase in rough handling was observed. Alcohol, on the other hand, adversely affected both performance measures and diminished the time taken to complete each lap. The authors concluded that the drug effects on performance were not dramatic since-no major differences were found between conditions with respect to observer ratings.

Casswell (1979) was the first who included a subsidiary task to simulate the demands for monitoring the environment. Thirteen males were tested in three treatment sessions receiving alcohol and marijuana treatments twice in each session and drove for 35 minutes after each treatment. Treatments included (1a) alcohol (0.10 g\% BAC) + placebo marijuana, (1b) placebo alcohol + marijuana (6.25 mg THC), (2a) double placebo, (2b) placebo alcohol + marijuana (6.25 mg THC), (3a) alcohol (0.05 g\% BAC) + marijuana (3.12 mg THC), and (3b) alcohol (0.05 g\% BAC) + marijuana (3.12 mg THC). Subjects' tasks included overtaking, driving on straight sections, through a hairpin bend, and through narrow gaps, while responding to road signals, traffic signals, and auditory signals in the subsidiary task. Alcohol alone and in 
combination with marijuana produced more coarse steering corrections, higher speed and increased lateral position variability. Marijuana alone was associated with lower driving speed and prolonged reaction times in the subsidiary task. Reaction times were also prolonged by the combination of marijuana and alcohol. The authors said that drivers under the influence of marijuana appeared to compensate for what they felt were the adverse effects of the drug by maintaining control effort, and decreasing speed to reduce the required rate of information processing. Alcohol, in contrast, appeared to produce more risky behavior.

Attwood et al. (1981) also employed normal driving tasks on a closed course. Eight males participated in a within-subjects design, receiving (1) double placebo, (2) alcohol $(0.08 \mathrm{~g} \%$ BAC) + placebo marijuana, (3) placebo alcohol + marijuana (150 $\mu \mathrm{g} / \mathrm{kg}$ THC), and (4) alcohol $(0.04 \mathrm{~g} \% \mathrm{BAC})+$ marijuana $(75 \mu \mathrm{g} / \mathrm{kg}$ THC). The driving tasks were performed on an airfield runway and included: maintenance of a constant lateral position and velocity, maintenance of a constant headway while following a lead car that varied in speed, bringing the car to a smooth stop at a traffic signal, and deciding whether or not to overtake a preceding vehicle in the presence of an approaching car. The latter maneuver was, however, not actually undertaken. Various measures, as speed, lateral position, acceleration and headway, were taken but the number of significant comparisons were no more than expected by chance. All measures were then subjected to a discriminant analysis that separated overall treatment effects. Overall driving performances after all drug treatments were significantly worse than following placebo when tested in this multivariate analysis. Smiley (1986) suspected that the lack of univariate effects was attributable to the low number of subjects and the lack of a subsidiary task.

Peck et al. (1986) assigned 84 subjects in equal proportions to four treatment conditions: (1) double placebo, (2) alcohol (0.08 g\% BAC) + marijuana placebo, (3) marijuana (19 mg THC) + alcohol placebo, and (4) both drugs combined. If these subjects could have inhaled all of the drug available in the cigarette, one weighing $70 \mathrm{~kg}$ (154 lb; population average) would have received a dose of about $270 \mu \mathrm{g} / \mathrm{kg}$. Because of the remaining butt, the actual THC dose probably never exceeded $250 \mu \mathrm{g} / \mathrm{kg}$. The subjects were tested four times in complete replications of a driving test battery beginning shortly after drug administration and continuing at hourly intervals thereafter. Ratings of the subjects' driving proficiency were obtained from driving licence examiners who rode with the subjects or observed them from static positions at points along the course; and, by California Highway Patrol officers who followed the subjects' vehicle in a police car. A computerized system within the subjects'. vehicle recorded their use of controls, speed and lateral position relative to course delineation. A risk acceptance test was included to measure the subjects' willingness and ability to drive through gaps wider and narrower than the vehicle. Other tasks involved stopping in response to signals, making a forced lane change and driving through pylons in a chicane. Finally, a standard police field sobriety examination and two standard laboratory tests (tracking and time estimation) were administered to the subjects outside of the vehicle. Several hundred measures of performance were obtained. No dramatic performance failures were reported as an effect of either drug or their combination. In general, the number of significant drug effects on particular measures were about what one might expect given the total number of statistical tests.

The investigators resorted, like Attwood et al., to multivariate statistical analysis of their data. Twelve performance measures were combined in discriminant analysis, which significantly separated the effects of each drug or their combination from placebo's. The THC effect was significant over all four replications of the tests, being greatest in the first trial. Alcohol's effect was greatest in the second trial and slightly greater than THC in every one. The combination 
of THC and alcohol produced significantly more impairment then did either drug alone in the first and third trials. Field sobriety checks by the police and ratings of the subjects' driving proficiency by experts failed to show any effect of THC, though these did reveal the effects of that drug in combination with alcohol. Practically the only indication of a serious effect of THC was provided by the officers following the subject's vehicle in a police car. They reported that they would have stopped the subject for suspicion of being intoxicated on $32 \%$ of all THC trials (alcohol $50 \%$, both drugs $60 \%$ ). But they also said they would have stopped $15 \%$ of the placebo treated subjects. This either indicates that the subjects were exceptionally poor drivers, or were made to appear so under conditions of the test, or that the officers were responding to cues that they ordinarily would have ignored in real driving conditions.

Smiley et al. (1987) tested the effects of marijuana $(0,100$ and $200 \mu \mathrm{g} / \mathrm{kg} \mathrm{THC})$ in combination with alcohol $(0.00$ and $0.05 \mathrm{~g} \% \mathrm{BAC})$ and alcohol alone $(0.08 \mathrm{~g} \% \mathrm{BAC})$ on driving in a closed-course study. Treatments were administered to groups of nine males over a three hour period in a party-like atmosphere in the evening. Subjects drove shortly after smoking as well as on the following morning. Driving tasks included maintenance of a constant lateraI position at $80 \mathrm{~km} / \mathrm{h}(50 \mathrm{mph})$, curve following, car following, route navigation, obstacle avoidance, and emergency decision making. Additionally, subjects had to perform a subsidiary task requiring visual monitoring. The high THC dose resulted in increased headway and headway variability. Alcohol alone at the $0.05 \mathrm{~g} \% \mathrm{BAC}$ level produced increased speed. Number of subsidiary task detections decreased at $0.05 \mathrm{~g} \%$ BAC but increased at $0.08 \mathrm{~g} \%$ BAC. Smiley's (1986) conclusion from her own and previous studies was as follows:

"...., marijuana does appear to impair driving behaviour. However, this impairment is mediated in that subjects under marijuana treatment appear to perceive that they are indeed impaired. Where they can compensate, they do, for example, by not overtaking; by slowing down and by focusing their attention when they know a response' will be required. Unfortunately, such compensation is not possible where events are unexpected or where continuous attention is required. Effects on driving behaviour are present shortly after smoking but do not continue for extended periods." (p. 133).

\section{General Conclusion}

The foremost impression one gains from reviewing the literature is that no clear relationship has ever been demonstrated between marijuana smoking and either seriously impaired driving performance or the risk of accident involvement. The epidemiological evidence, as limited as it is, shows that the combination of THC and alcohol is over-represented in injured and dead drivers and more so in those who actually caused the accidents to occur. Yet there is little if any evidence to indicate that drivers who have used marijuana alone are any more likely to cause serious accidents than drug free drivers. To a large extent, the results from driving simulator and closed-course tests corroborate the epidemiological findings by indicating that THC in single inhaled doses up to $250 \mu \mathrm{g} / \mathrm{kg}$ has relatively minor effects on driving performance, certainly less than BACs in the range $0.08-0.10 \mathrm{~g} \%$. 


\section{CHAPTER 2 - GENERAL METHODS}

Before presenting the designs and results of the individual studies in separate chapters, it seems appropriate to describe the studies of the program and certain procedures that were common to all. These were subject recruiting, compliance with ethical and legal standards, screening for the presence of other illicit drugs and alcohol, and blood sampling procedures and quantitative analyses.

\section{DESCRIPTION OF A 4-STUDY PROGRAM}

The present relationship between drivers' use of marijuana and other substances containing the psychoactive drug (THC) and their involvement in traffic accidents is exceedingly obscure. For a variety of practical reasons, epidemiological research has failed to define that relationship in a manner approximating the demonstration of alcohol's effects on traffic safety (Terhune, 1986). The classical approach of first showing that a drug is actually causing traffic accidents before determining how has simply failed for THC.

Yet abundant experimental evidence exists to show that some doses of THC can impair skills deemed important for safe driving. Unfortunately, most of it is of dubious relevance to the actual driving situation: nearly all of the studies on THC's behavioral effects were accomplished using laboratory tests that are not directly related to actual car driving. If previous experience is any guide, little of crucial importance will emerge from experimental research until it is conducted in a more "real world".

Closed-course driving studies have shown that marijuana can impair driving performance but it is unknown to what extent these effects translate into driving performance in the real world. Only one study has been conducted in real world (city driving; Klonoff, 1974). This study demonstrated that subjects perform less competently when under the influence of marijuana, but the scoring method was questioned by others as regards its relationship to safe driving performance.

Bearing these limitations of previous studies in mind a program was set up to determine the dose-response relationship between marijuana and objectively and subjectively measured aspects of real world driving; and, to determine whether it is possible to correlate driving performance impairment with plasma concentrations of the drug or a metabolite. These goals are the same as those of many unsuccessful investigations in the past. Yet none before has gone so far in seeking to achieve them in the environment where the "drugs and driving" problem actually exists. In the present studies, a variety of driving tasks were employed, including: maintenance of a constant speed and lateral position during uninterrupted highway travel, following a leading car with varying speed on a highway, and city driving. The purpose of applying different tests was to determine whether similar changes in performance under the influence of THC occurs in all thereby indicating a general drug effect on driving safety.

The program consisted of one minor and three major studies; a series of separate but interdependent experiments that successively approached driving reality. This approach was necessary to ensure subject safety throughout the program. The program started with a pilot 
study (Chapter 3), conducted in a hospital under strict medical supervision, to identify THC doses that recreational marijuana users were likely to consume before driving.

The first driving study (Chapter 4) was executed on a closed section of a public highway. The major goal was to determine the dose-response and dose-response-time relationship between marijuana (three different THC doses, and placebo) and road tracking precision as measured by the "weaving" motion of the subject's vehicle during uninterrupted highway travel. Results of this are compared to those from a previous study undertaken by the investigators to measure the effects of different blood alcohol concentrations on driving performance in essentially the same test situation (Louwerens et al., 1987). A practical purpose was to determine whether the drug's effects as measured in a standard driving test were of a magnitude that would safely allow apphication of the same test and others on public roads in traffic.

Upon completion of this study with the demonstration that THC's effects could be safely controlled, a second driving study (Chapter 5) was conducted to come a step closer to driving reality than its predecessor. The methods applied were, with the addition of a car following test, the same as those used in the first driving study. However, driving tests were now conducted on a highway in the presence of other traffic. The greatest discretion was employed in designing this study to reach limited objectives. We choose a conservative approach which closely follows that used to determine the tolerability of medicinal drugs in human pharmacological research. It is to test THC's effects on actual driving performance in an ascending dose series. The ultimate goal was to define the THC dose (or plasma concentration) limit which separates low and high risk driving performance impairment by approaching it from the bottom up.

Yet normal driving is far more complex and varied than simply to maintain a safe lateral

position and headway during uninterrupted travel on a highway. A THC dose having no effect on these parameters might still impair driving performance in more complex urban driving situations. For this reason the program then proceeded into the third and final driving study (Chapter 6) which involved tests conducted in high density urban traffic. The highest dose which had no significant effect on highway driving in the previous study was given to subjects who would now operate in an urban driving test. This provided an opportunity to measure a far broader range of driving performance. If no effect were again observed, the generality of the dose-effect relationship would be strengthened. But if a new kind of impairment were observed, the conclusion would have to be that the dose effect relationship can not be validly used to define the effects of THC on driving performance, in general. The nature of the new impairment would provide insight into the kinds of traffic safety problems that may be first to appear as a consequence of the drug's effects. A second group also participated in this study and undertook the same driving test, but then after drinking alcohol (reaching an average BAC of $0.04 \mathrm{~g} \%$ ), and a placebo. This was done for two reasons; first, the alcohol condition served as a control whether the employed tools to assess driving performance were sensitive; and, secondly, it made a comparison possible between low doses of alcohol and THC.

\section{SUBJECTS}

The ideal subjects would be male and female marijuana users whose consumption of the drug represents that of the majority in that particular population. Van der Wal's (1985) data for the oldest group (17-18 years) in his sample of present Dutch cannabis users indicate that about $56 \%$ 
of the males and females have a usage frequency of more than once per month and less than daily. This usage frequency was considered as the first selection criterion.

The second criterion was that the users should also be experienced drivers in possession of a driver's licence. Subjects must have driven at least $5,000 \mathrm{~km}(3,108 \mathrm{mi})$ per year over the previous three years. This criterion was, however, not always met because of the difficulties in recruiting subjects.

As the third criterion the users should have indicated on a questionnaire that they had driven within one hour after smoking cannabis at least once within the preceding year. These users not only possess the requisite driving experience under the influence of marijuana, they also constitute the "drivers at risk". In addition, the application of this criterion avoided the ethical dilemma of requiring subjects to accept a risk which they would otherwise avoid.

As a fourth criterion, the subjects should agree to refrain from their normal marijuana use for at least five days prior to their participation in any test.

Other inclusion criteria were as follows: age 21-40 years; normal (corrected or uncorrected) binocular acuity (i.e. 20/25 Snellen acuity, or better); body weight within the $85^{\text {th }}-115^{\text {th }}$ percentile range according to the 1983 table from the Metropolitan Life Insurance Company; and, Dutch nationality. The latter criterion was a condition set by the Dutch Ministry of Health which has no authority to permit the use of an illicit drug by foreign nationals.

Exclusion criteria included the following:

1. No history of treatment for drug or alcohol abuse or addiction and no reasonable possibility of dependence occurring as the result of participation in the investigation.

2. No record of arrests or conviction for drug trafficking.

3. No history of psychiatric or organic brain disorders.

4. No overt signs of cardiovascular,-respiratory, renal, hepatic, metabolic or neuromuscular disorders and no history of serious disorders of this type.

5. No current use of any psychoactive medication (tranquilizers, antidepiessants etc.)

6. For females, no pregnancy or any reasonable probability that pregnancy might occur during participation in the investigation.

Some subjects volunteered spontaneously after reading about the planned study in newspapers. Other volunteers for the first two studies were primarily obtained from among the local population of marijuana users by means of advertisements. Both the second and the third driving study required new samples of subjects. In these cases it was more difficult to recruit subjects since advertisements could not be placed where they might attract the attention of news media. The desire to avoid attention was fostered by a need to ensure subjects' anonymity and avoid the media's interference with data collection involving driving in traffic on public highways and city streets. Subjects were therefore recruited in the last two studies mainly by contacts obtained from subjects from the preceding ones. Admittedly this procedure is not the best to acquire independent samples but was necessary for practical reasons.

Volunteers were screened in two stages; first from their responses to a combined cannabis use, driving experience and medical history questionnaire; and secondly, on the basis of an interview and physical examination. Furthermore law enforcement authorities were contacted, with the volunteers' consent, to verify that they had no previous arrests or convictions for drug trafficking.

Subjects were instructed to sleep normally on the nights before test days. Alcohol consumption was prohibited for 24 hours before tests, and consumption of beverages containing 
caffeine, for 2 hours beforehand. Those who smoked tobacco were advised that this would also be prohibited for one hour before testing until its completion.

\section{COMPLIANCE WITH ETHICAL AND LEGAL STANDARDS}

All studies described in this report complied with the code of ethics on human experimentation established by the Declaration of Helsinki (1964) as amended in Tokyo (1975), Venice (1983), and Hong Kong (1989). This implies that the volunteer subjects were fully informed of all procedures, possible adverse reactions to drug treatments, legal rights and responsibilities, expected benefits of a general scientific nature, and their right for voluntary termination without penalty or censure. All subjects gave their informed consent, in writing. Their anonymity was and will be maintained in all communications from the project. The investigators provided for continuous medical supervision and emergency medical treatment during the studies. Approvals for individual studies were separately obtained from the University's Medical Ethics Committee.

Before the program started an Independent Advisory Committee was formed whose function was to ensure that the program proceeded in accordance with all medical and legal standards. This committee comprised the Assistant District Attorney, the Municipal Traffic Attorney for the City of Maastricht, a member of the University's Medical Ethical Committee, and the Dutch Regional Inspector for Public Health (Drugs). A permit for obtaining, storing and administering marijuana was obtained from the Dutch Drug Enforcement Administration.

Subjects were accompanied on every driving test by a licensed driving instructor experienced in supervising subjects who operated under the influence of medicinal drugs in previous studies. The instructor's sole task was that of monitoring ride safety. Redundant control system in the test vehicle was available for controlling the car if emergency situations should arise. However, the primary guarantor of the subject's safety was the subject himself/herself. The subject, like any licensed Dutch driver, had the legal responsibility to stop driving when feeling "under the influence" to the point where he/she could no longer be sure of his/her ability for safely controlling the vehicle. Subjects in this investigation were reminded of their responsibility and urged not to undertake any test, or to stop driving during a test in progress, if they felt incapable of driving safely. Subjects were always transported to and from their appointments and were strictly instructed not to operate their own vehicles for a period of 12 hours after having received the experimental treatment.

\section{SCREENING FOR THE PRESENCE OF OTHER ILLICIT DRUGS AND ALCOHOL}

Though it seemed unlikely that subjects would regularly resort to using other illicit drugs or alcohol prior to controlled marijuana smoking and testing, the possibility could not be definitely excluded without testing the subjects for the presence of these drugs. Therefore they were informed beforehand of the intention to.. obtain urine and breath samples which would be analyzed for the presence of prohibited agents.

Each subject was required to submit a urine sample immediately upon arrival at the test site. Samples were later assayed qualitatively for the following drugs (or metabolites): cannabinoids, benzodiazepines, opiates, cocaine, amphetamines and barbiturates. In addition a breath sample was analyzed on the spot for the presence of alcohol using a Lion S-D3 Breath-Alcohol 
Analyzer. The urine and breath sample screening procedures were employed in all studies in the program.

Drugs other than cannabinoids were found in urine of four subjects. In the pilot study, the urine of two subjects was positive for benzodiazepines; and, of one subject for barbiturates. Analyses of six urine samples obtained from these subjects during the successive driving study failed to show the presence of these drugs. Since all urine samples from both the pilot and first driving study were analyzed after completion of the latter, the failure to detect the drugs in samples obtained during the driving study indicates that they did not abuse these drugs. Upon questioning, all three subjects denied that they bad taken these drugs. Since no urine or plasma was left from these subjects, it was, however, not possible to check whether the results were false positives. Data obtained from these subjects in the pilot study were not excluded from the statistical analyses. One subject's urine, obtained prior to smoking in the $200 \mu \mathrm{g} / \mathrm{kg}$ condition in the first driving stuidy, was positive for cocaine. Upon questioning, the subject replied that some friends had surreptitiously administered him cannabis cake and cocaine the day before. Assuming that the drugs' effects had dissipated the next day, these subject's data were also not excluded from statistical analyses.

\section{BLOOD SAMPLING AND QUANTTTATIVE ANALYSES}

Blood samples were taken by venepuncture. Two $10 \mathrm{ml}$ aliquots were obtained in every case. These were heparinized and centrifuged within 30 minutes. Plasma was placed in frozen $\left(-20^{\circ} \mathrm{C}\right)$ storage prior to analysis. The quantitative chemical analysis of THC and THC-COOH in plasma was performed by gas chromatography/mass spectrometry (GC/MS) using deuterated cannabinoids as internal standards (Möller et al., 1992). Of the many analytic techniques available at present, GC/MS is the reference method of choice (Cook, 1986). Applying this method, the detection limits for THC and THC-COOH were about 0.3 and $3.0 \mathrm{ng} / \mathrm{ml}$, respectively. THC and THC-COOH concentrations in plasma will further be abbreviated to [THC] and [THC-COOH].

If the urine analysis (above) was positive for cannabinoids, plasma taken before smoking was also analyzed to quantitatively determine [THC] and [THC-COOH]. Subjects with detectable THC in pre-smoking plasma are shown in Table 2.1. In the pilot and first driving study, THC was detected in each pre-smoking plasma sample from two subjects and in one sample from another subject, namely prior to smoking in the $200 \mu \mathrm{g} / \mathrm{kg}$ condition. In the second driving study, THC was detected in one sample from one male and in five out of six samples from another male. In the city driving study, THC was not detected in any pre-smoking sample.

It seems obvious that those subjects; who had detectable [THC] before smoking, did not comply with the instruction to abstain from cannabis consumption for at least five days prior to the trial. They all had long histories (at least 7 years) of cannabis experience and were frequent (at least twice a week) users. Gieringer (1988) reports that THC may persist in the blood of chronic smokers at levels up to $4.0 \mathrm{ng} / \mathrm{ml}$ after 48 hours. It therefore remains an open question when their latest consumption was or whether they were impaired upon arrival at the laboratory.

The same pattern of pre- to post-smoking values as shown in Table 2.1 was observed in the other subjects, i.e. [THC] and [THC-COOH] -increased considerably after smoking the administered marijuana cigarettes and not following placebo. Therefore, these subjects' data were not excluded from the statistical analyses. 
Table 2.1 Pre-smoking and post-smoking [THC] in subjects with detectable THC in pre-smoking plasma samples.

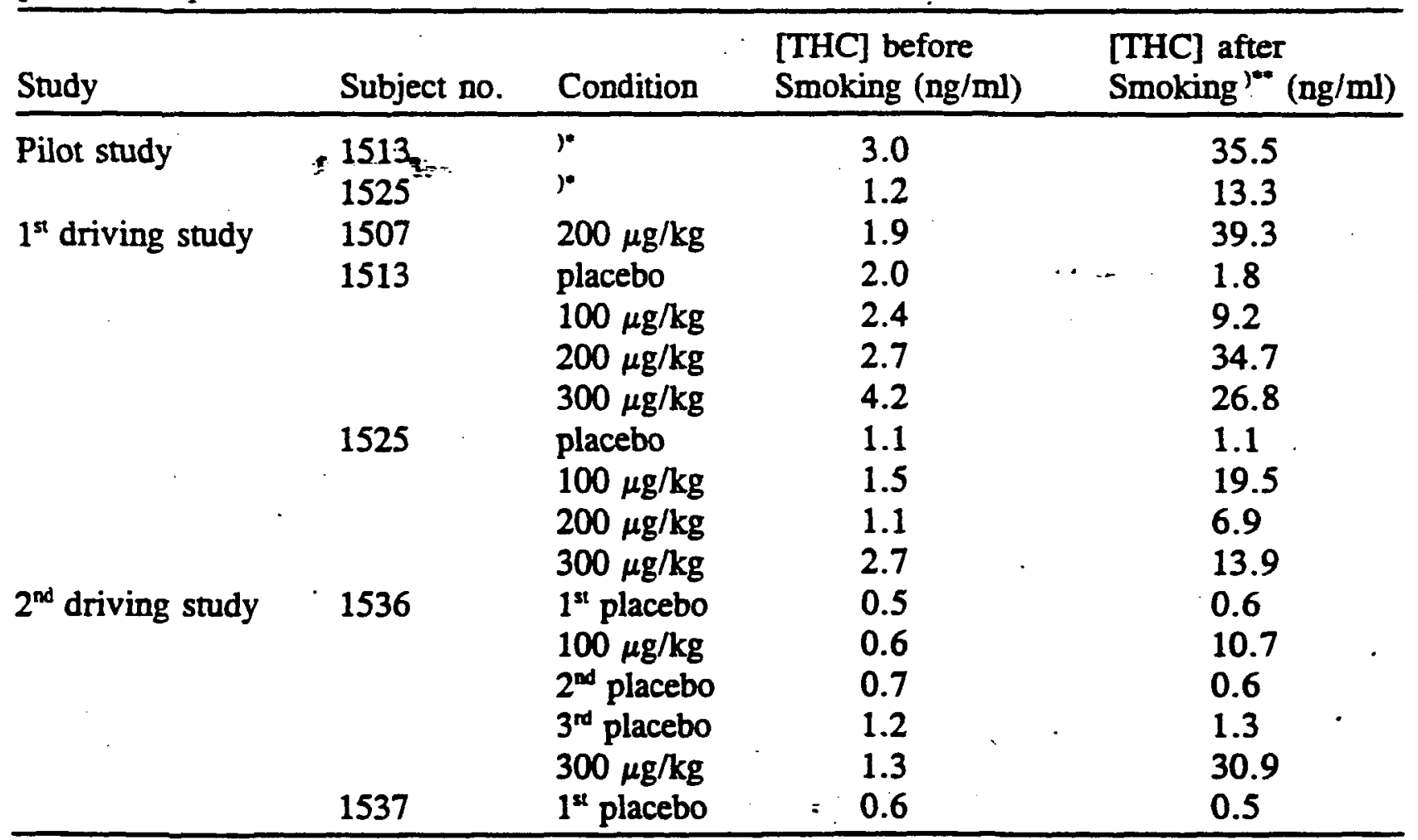

r. only one condition (smoking until the desired effect was achieved).

sampling time was 40,30 and 35 minutes after initiation of smoking in the pilot, first and second driving study, respectively.

Fifty percent of the pre-smoking plasma samples obtained from subjects in the pilot and first driving study, whose urine tests were negative for cannabinoids, were also analyzed. These analyses were performed to examine whether any false negative urine analyses had occurred. Results showed that none of these samples contained detectable [THC] or [THC-COOH]. From these results it was inferred that in subsequent studies pre-smoking blood samples need only be taken if the urine test for cannabinoids were positive. 


\section{CHAPTER 3 - PILOT STUDY TO SELECT THC DOSES}

\section{INTRODUCTION}

Doses used in all previous studies of inhaled THC have been selected without consulting the subjects beforehand to determine whether these realistically approximated doses they commonly use. In the opinion of several experts.(Moskowitz, 1985; Chesher, 1986; Peck et al., 1986) this has resulted in the selection of maximum doses for experimental purposes that are considerably less than those used for recreational pursuits. One could arbitrarily select higher doses but with the risk of erring in the opposite direction. A dose of, say $300 \mu \mathrm{g} / \mathrm{kg}$, might be higher than any taken at one time by street users or, at any rate, higher than one they might take and then drive. If we were to select unrealistically high doses these could result in bizarre and potentially dangerous reactions in even the most controlled driving test. To avoid arbitrarily selecting the wrong maximum dose, it seemed necessary to consult the subjects in the context of a "clinical" pilot study.

The pilot study's major purpose was therefore to establish the maximum dose for subsequent driving studies. Yet it provided several opportunities for obtaining valuable information about THC's pharmacokinetics and its pharmacodynamic effects after marijuana smoking. Blood samples were repeatedly taken for measuring [THC] and [THC-COOH]. The subjects repeatedly performed certain simple laboratory tests, estimated their levels of intoxication and indicated their willingness to drive under several specified conditions of urgency. In addition, heart rate was measured at these times. The secondary purpose was that of specifying relationships between [THC] and [THC-COOH] with changes in the other physiological, performance or subjective variables.

\section{METHODS}

\section{Subjects}

Twenty-four healthy volunteers, 12 males and 12 females, volunteered to participate in this study. They were screened as described in Chapter 2. Groups of six were treated and tested per inght. Sessions were conducted in the evening between 19.00 and 24.00 hours and subjects smoked and were tested at staggered intervals of 10 minutes. GC/MS analysis of plasma obtained from one male revealed trivial amounts of values THC in the first. and second samples after smoking but none thereafter, and no THC-COOH in any sample. It was concluded that this subject had not inhaled smoke so his data were excluded from further analyses. Characteristics of the remaining 23 subjects are given in Table 3.1. T-tests for independent samples showed that males were more experienced smokers than females $(p<.044)$. Males' driving experience was nearly significantly greater than females' ( $p<.056 \& .089$ for number of years and $\mathrm{km}(\mathrm{mi})$, respectively). 
Table 3.1 Mean $\pm S D$ (range) of subjects' characteristics.

\begin{tabular}{lrr}
\hline & $\begin{array}{r}\text { Males } \\
(\mathrm{N}=11)\end{array}$ & $\begin{array}{r}\text { Females } \\
(\mathrm{N}=12)\end{array}$ \\
\hline Age (yrs) & $27.0 \pm 4.6(22-38)$ & $24.6 \pm 2.9(21-31)$ \\
Weight (kg) & $69.8 \pm 10.3(56.5-87.0)$ & $66.6 \pm 9.1(52.0-79.0)$ \\
Weight (lb) & $154 \pm 23(125-192)$ & $147 \pm 20(115-174)$ \\
Smoking Experience (yrs) & $9.2 \pm 5.8(1-23)$ & $5.0 \pm 3.3(2-14)$ \\
\# Joints/Month & $5.9 \pm 3.7(1-14)$ & $5.8 \pm 5.4(1-20)$ \\
Driving Experience (yrs) & $8.5 \pm 5.7(3-23)$ & $4.8 \pm 2.7(1-12)$ \\
Driving Experience (km x 1000) & $121 \pm 118(15-360)$ & $54 \pm 54(5-175)$ \\
Driving Experience (mi x 1000) & $75 \pm 73(9-224)$ & $34 \pm 34(3-109)$ \\
\# of Subjects Having Driven within & & \\
1 Hour following Cannabis Consumption: & 1 & 9 \\
less than 5 times & 10 & 3 \\
5 times or more & & \\
\hline
\end{tabular}

\section{Smoking Procedures}

Marijuana cigarettes were supplied by the U.S. National Institute on Drug Abuse (NIDA). The cigarettes had an average weight of $767 \mathrm{mg}$ and contained $2.57 \%$ or about $20 \mathrm{mg}$ THC. Cigarettes were humidified by placing them at room temperature overnight in a desiccator containing a small quantity of saturated sodium chloride solution. This procedure raised the moisture content of the cigarettes from $10 \%$ to $15 \%$, on the average. The subjects were allowed to smoke part or all of the THC content in three cigarettes until achieving the desired psychological effect. Cigarettes were smoked through a plastic holder, in a fashion determined by the subject. The only requirement was to smoke continuously for a period not exceeding 15 minutes. When subjects voluntarily stopped smoking, cigarettes were carefully extinguished and retained for subsequent gravimetric estimation of THC consumed $(2.57 \%$ of the difference between the weight of the original cigarette and the remaining unsmoked portion). This method of estimating THC amounts consumed is based upon the assumption that THC is equally distributed over the entire cigarette. Perez-Reyes et al. (1982) analyzed THC concentrations in the unsmoked portions of marijuana cigarettes of three different potencies and indeed found that they were identical to those in the unlit cigarette.

\section{Measurements}

A test battery which lasted 20 minutes took place before smoking and was repeatedly administered at 30, 90, 150 and 210 minutes after initiation of smoking. The battery consisted of:

1. The Critical Tracking Test (CTT). This test, described in detail by Jex et al. (1966), was implemented on a IBM-compatible MS-DOS computer and measures the subject's ability to control a displayed error signal in a $1^{\text {st }}$-order compensatory tracking task. Error appears as horizontal deviation of a cursor from midpoint on a horizontal, linear scale. Compensatory joy-stick movements null the error by returning the cursor to the midpoint. The frequency 
of cursor deviations, and therefore its velocity, increase as a stochastic, linear function of time. The frequency at which the subject loses control is called the critical frequency $\left(\lambda_{c}\right.$, expressed in $\mathrm{rad} / \mathrm{s}$ ). Theoretically, $\lambda_{t}$ is the reciprocal of the operating delay lag in human closed-loop manual control. The test included 5 trials of which the lowest and highest score were removed; the average of the remaining scores was taken as the final test score. Total test time duration was approximately 5 minutes.

2. Questionnaires. Subjects were required to rate their feeling of "high" as a percentage of the maximum ever experienced, and to indicate certain feelings of present cognitive and emotional state using the 16-item visual-analog scale developed and standardized for drug research by Bond and Lader (1974). Scores on the latter scales were grouped to form three cluster scores for measuring the corresponding factors: alertness, contentedness and calmness which will be expressed as percentage of the maximum. In addition, the subjects' willingness to operate a motor vehicle was assessed by asking them to declare whether they would attempt to drive for a set distance if the reasons were: $A$. unimportant though gratifying, such as for transporting a friend to another party; $B$. important but avoidable, such as for transporting a mildly sick friend home when he would otherwise have to call a taxi; and $C$. urgent, such as transporting a severely sick infant to the hospital. These ratings were made by subjects immediately after termination of smoking and after conclusion of the CTT. All questionnaires are enclosed in Appendix A.

3. Heart Rate. Heart rate was measured by counting the number of beats per minute immediately after completion of the questionnaires.

4. Hand Steadiness Test. Thereafter, hand steadiness was measured from the number of side contacts occurring as the subject attempted to hold a $1 \mathrm{~mm}(0.04 \mathrm{in})$ stylus for 15 seconds within each of five circular holes with successively diminishing diameters $(3.90,3.05,2.70$, 2.20 and $1.85 \mathrm{~mm}$, respectively; or, $0.15,0.12,0.11,0.09$ and $0.07 \mathrm{in}$ ). Subjects were allowed to rest their hand on the table. The test score was defined as the total number of contacts of the stylus with any side. Since the distribution of subjects' scores were skewed, a square root transformation was applied to normalize data. The test lasted about 3 minutes.

5. Blood Sampling. A blood sample was taken by means of a venepuncture ten minutes after the beginning of the test battery. The sample ( 2 aliquots containing $10 \mathrm{ml}$ blood apiece) was heparinized and centrifuged, and plasma was placed in frozen $\left(-20^{\circ} \mathrm{C}\right)$ storage prior to analysis for THC and its major metabolite THC-COOH. Blood samples were taken before and at 40,100,160 and 220 minutes after initiation smoking.

Subjects were familiarized with the questionnaires and practiced the CTT and hand steadiness test on three separate occasions during the weeks prior to the test night until they reached a steady performance level.

\section{Data Analysis}

Parametric data were analyzed as. follows, All data including baseline values entered a repeated measures multivariate analysis of variance (MANOVA) with Sex as a between-groups and Time as a within-subjects factor and the criterion for significance set at .05 . If a significant Time effect was found, a repeated measures univariate analysis of variance (ANOVA) was performed to separately compare each post-smoking measurement with baseline. Sex was not a factor in these ANOVA's unless MANOVA had revealed a significant Sex by Time interaction. Individual 
comparisons with baseline were not possible for perceived "high" ratings and plasma levels of the drug since baseline values were zero in most cases. Instead, if MANOVA revealed a significant Time effect, the data were further analyzied in an ANOVA trends analysis to determine the significance of linear, quadratic and cubic components. In the figures, the mean of the variable is depicted by a point and its standard error (SE) by the height of the vertical line above the point.

The subjects' expressions of willingness to drive were made on the basis of a dichotomous decision and could not for that reason be analyzed in the same manner as other variables. These data were therefore analyzed using Cochran's Q-statistic to determine if the proportion of subjects willing to drive changed over time. If they did significantly, separate changes from baseline were tested by McNemar's sign-test.

Criterion for statistical significance in individual comparisons was adjusted by means of the "Sequential Bonferroni" correction (Overall and Rhoades, 1987) to retain a constant type I error probability of .05 across the entire set of comparisons. This means that for the largest of four differences tested at once, $p$ had to be less than $.05 / 4=.013$ to be judged significant. For the second, it had to be $<.05 / 3=.017$; for the third, $<.05 / 2=.025$; and for the smallest difference, $p<.05$.

Significant Sex effects were generally absent. Results pertaining to differences between the sexes are therefore only reported for the exceptional cases where the differences were significant.

Two types of correlations were calculated to determine the linear relationship between two variables; i.e., the inter-subject and intra-subject correlation. The first is the most commonly used; it is obtained from pairs of variables measured in a group of subjects. In this study, for example, the correlation between [THC] and $\lambda_{l}$, the tracking performance score, was calculated for all subjects at each sampling time separately. This resulted in four correlations (one at $t=40$, 100,160 and $220 \mathrm{~min}$ ) obtained from 23 subjects $(\mathrm{N}=23)$. These correlations were tested for significant departures from zero by t-test.

The intra-subject correlation, on the other hand, is the correlation between pairs of valriables within one subject. In the present study, for example, the correlation between [THC] and $\lambda_{c}$ was also. calculated for each subject, across all sampling times, separately. This resulted in 23 correlations (one from each subject) obtained from four repeated measurements $(N=4$; baseline values were excluded). These were transformed into Fisher's $z$-scores and then averaged across subjects yielding $z_{a v}$, which was tested for significant deviation from zero by t-test, and transformed back to $r$.

Interpretations of these two types of correlation are not the same. If, in the present study, a perfect inter-subject correlation $(r= \pm 1.0)$ between [THC] and $\lambda_{r}$ existed, it would mean that one can perfectiy predict $\lambda_{\tau}$ of a particular subject from the knowledge of his/her [THC]. Usually, however, inter-subject correlations are much lower; and, the closer to zero, the more unreliable the predictions become.

A high average intra-subject correlation means that, on the average, scores on two variables are closely related within a subject, but not necessatily between subjects. Thus, a highly negative average intra-subject correlation between $[\mathrm{THC}]$ and $\lambda_{c}$ (lower scores indicating poorer performance) would mean that, within a subject, higher plasma levels of THC are associated with poorer tracking performance. Yet this does not imply that, if two subjects are compared, the one with the higher plasma levels performs worse. That would only be the case if both the average intrasubject and the intersubject correlations were strongly negative. 


\section{RESULTS}

\section{Consumed THC}

Six subjects consumed one cigarette, thirteen smoked two and four smoked three. Total THC amounts consumed are given in Table 3.2. Statistical analyses failed to reveal a significant difference between the sexes. It should be noted that these amounts of THC represent both the amount inhaled and the portion that was lost through pyrolysis and side-stream smoke during the smoking process.

Table 3.2 Mean, median and range of amounts of THC consumed, both in absolute values and relative to bodyweight (BW).

\begin{tabular}{lllllll}
\hline & \multicolumn{2}{l}{ THC consumed $(\mathrm{mg})$} & \multicolumn{3}{c}{ THC consumed per kg BW $(\mu \mathrm{g} / \mathrm{kg})$} \\
& mean & median & range & mean & median & range \\
\hline Males $(\mathrm{N}=11)$ & 22.3 & 18.6 & $14.7-35.2$ & 324 & 292 & $203-524$ \\
Females $(\mathrm{N}=12)$ & 19.4 & 18.9 & $11.3-28.2$ & 293 & 292 & $194-440$ \\
All $(\mathrm{N}=23)$ & 20.8 & 18.8 & $11.3-35.2$ & 308 & 292 & $194-524$ \\
\hline
\end{tabular}

\section{Plasma Concentrations of the Drug}

Mean, median and range of [THC] and [THC-COOH] at each sampling time are shown in Tables.3.3 and 3.4. Maximum [THC] was found in the first sample after smoking at $t=40$ minutes. Males had somewhat higher [THC] and much higher [THC-COOH] values than females; the difference between both sexes was however rather constant over time, except for [THC] at $t=40$ where the difference was quite profound. These observations were confirmed by MANOVA that showed a significant Sex effect for both [THC] and [THC-COOH] $\left(\mathrm{F}_{1.21}=4.3\right.$ \& 9.79; $p<.05 \& .005$, respectively), but no significant Time by Sex interaction. Since consumed THC amount did not differ between both sexes, the conclusion must either be that males were more efficient smokers than females, or that they absorbed the active ingredient differently.

There was a significant Time effect for both [THC] and [THC-COOH] $\left(\mathrm{F}_{3,19}=14.79 \& 11.70\right.$, respectively; $p<.001$ in both cases). Univariate trend analysis revealed that both linear, quadratic as well as cubic functions fitted the trend in [THC] over time significantly $\left(\mathrm{F}_{1.21}=44.56,38.95 \& 29.23 ; p<.001, .001 \& .001 ; p_{c}=.017, .025 \& .05\right.$, respectively) due to a rapid decline of [THC] in plasma after the first sample. THC-COOH changes over time were only significantly fitted by a linear trend $\left(F_{1.21}=26.92 p<.001 ; p_{c}=.017\right)$.

The relation between consumed THC, relative to body weight, and [THC] was examined by calculation of inter-subject correlations (intra-subject correlations could not be determined from the data because each subject smoked only one dose of THC). These analyses showed moderate inter-subject correlations between both parameters at each sampling time, namely $0.42(p<.05)$, 0.34 (ns), $0.42(p<.05)$ and $0.45(p<.05)$. Yet inspection revealed that the apparent strength $\cdots$ of these correlations was almost totally attributable to two males who had consumed the greatest amounts of THC (486 and $524 \mu \mathrm{g} / \mathrm{kg}$ ) and had also very high plasma levels of THC (45.9 and $35.5 \mathrm{ng} / \mathrm{ml}$, respectively). When $\log$ values of [THC] and consumed THC were used, to 
normalize the distributions, the correlations were small and not significant. There were no differences between males and females with respect to these correlations.

Table 3.3 Mean, median and range of [THC] in $\mathrm{ng} / \mathrm{ml}$.

\begin{tabular}{llllll}
\hline & & $t=40$ & $t=100$ & $t=160$ & $t=220$ \\
\hline \multirow{2}{*}{ Males $(\mathrm{N}=11)$} & mean & 17.7 & 5.8 & 2.8 & 1.7 \\
& median & 13.3 & 4.5 & 2.3 & 1.3 \\
& range & $6.7-45.9$ & $2.5-15.2$ & $1.2-6.8$ & $0.7-5.1$ \\
& & & & & 0.7 \\
Females $(\mathrm{N}=12)$ & mean & 9.9 & 3.0 & 1.4 & 0.7 \\
& median & 7.0 & 2.7 & 1.0 & 0.6 \\
& range & $-3.3-19.3$ & $0.3-6.8$ & $0.5-3.2$ & $0.0-1.8$ \\
& & & & & \\
All $(\mathrm{N}=23)$ & mean & 13.6 & 4.3 & 2.1 & 1.2 \\
& median & 9.9 & 3.5 & 1.4 & 0.8 \\
& range & $3.3-45.9$ & $0.3-15.2$ & $0.5-6.8$ & $0.0-5.1$ \\
\hline
\end{tabular}

Table 3.4 Mean, median and range of [THC-COOH] in $\mathrm{ng} / \mathrm{m}]$.

\begin{tabular}{llllll}
\hline & & $t=40$ & $t=100$ & $t=160$ & $t=220$ \\
\hline \multirow{2}{*}{ Males $(\mathrm{N}=11)$} & mean & 33.9 & 28.1 & 25.3 & 20.8 \\
& median & 25.6 & 19.6 & 18.3 & 13.5 \\
& range & $12.9-96.4$ & $12.8-72.4$ & $9.5-63.5$ & $8.0-67.0$ \\
& & & & & \\
Females $(\mathrm{N}=12)$ & mean & 12.4 & 9.5 & 8.3 & 5.6 \\
& median & 9.6 & 8.2 & 7.5 & 5.5 \\
& range & $3.3-39.9$ & $0.5-26.8$ & $3.0-15.8$ & $0.0-13.0$ \\
& & & & & \\
All $(\mathrm{N}=23)$ & mean & 22.7 & 18.4 & 16.4 & 12.8 \\
& median & 17.3 & 13.9 & 13.6 & 10.1 \\
& range & $3.3-96.4$ & $0.5-72.4$ & $3.0-63.5$ & $0.0-67.0$ \\
\hline
\end{tabular}

These results indicate that the between-subject variability in [THC] is not related to the betweensubject variability in the consumed amount of THC; in other words, information about [THC] and the time of blood sampling after smoking, of a particular individual, does not reveal how much that subject smoked, nor vice versa.

\section{Perceived "high"}

Mean subjective ratings of "high" are shown in Figure 3.1. The subjects consistently reported their peak subjective reaction as being about $70 \%$ of the greatest ever experienced. This was achieved shortly after smoking. Their subjective feelings declined, again in a highly consistent 


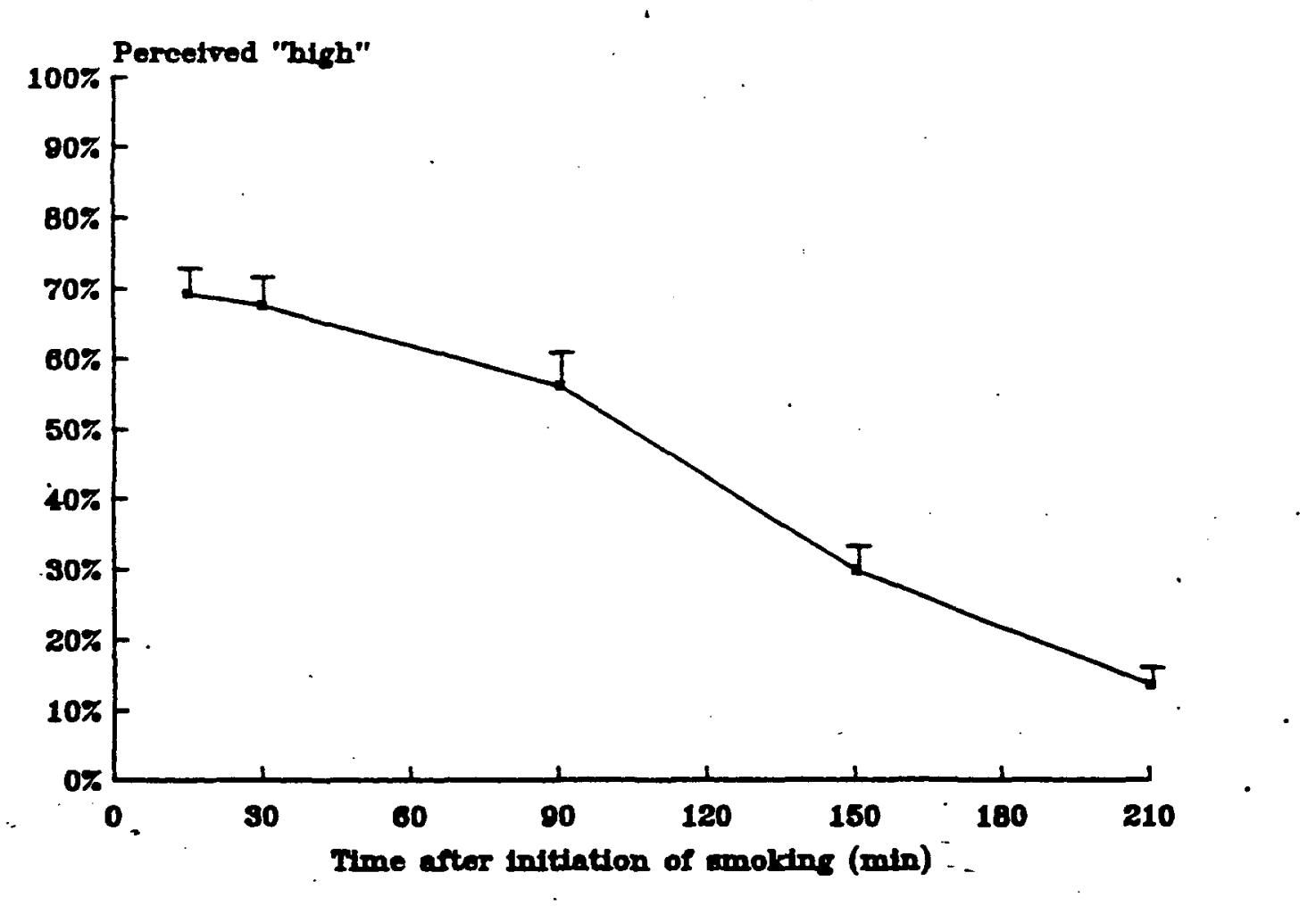

Figure 3.1 Mean (+SE) perceived "high" by Time.

manner between subjects; until arriving at a level less than $15 \%$ of the greatest ever experienced, at a time $31 / 4$ hours after smoking.

MANOVA revealed a significant Time effect $\left(F_{4,18}=49.18 ; p<.001\right)$. Trend analysis confirmed the impression from the data that the downward trend is best fitted by a linear function $\left(F_{1.21}=201.92 ; p<.001\right)$.

\section{Willingness to Drive}

Figure 3.2 displays percentages of the subjects that declared to be willing to drive under different circumstances of a gradually more compelling nature (A. unimportant though gratifying; $B$. important but avoidable; and, $C$. urgent). Only about $20 \%$ of the subjects declared their willingness to drive for relatively unimportant reasons $(A$ and $B)$, but approximately $80 \%$ declared that they would do so for an urgent reason $(C)$, while experiencing the peak subjective reaction. Willingness to drive gradually rose to about $70 \%$ for unimportant reasons, and $100 \%$ for an urgent reason, by the end of the test session.

Cochran's test revealed that time-related changes in willingness to drive were statistically significant in all three circumstances $\left(Q_{d f-5}=46.62,44.62 \& 15.17 ; p<.001, .001 \& .01\right.$ for situations $A, B \& C$, respectively). McNemar's test showed that the percentage of subjects willing to drive under circumstance $A$ was significantly different from baseline until the end of the test session $\left(p<.001, .001, .001, .002 \& .02 ; p_{c}=.01, .013, .017, .025 \& .05\right)$, while under circumstance $B$ changes were only significant until $2 \frac{1}{2}$ hours after smoking $(p<.001, .001 \&$ 
$\left..004 ; p_{c}=.01, .013 \& .017\right)$. McNemar's test failed to detect any significant change after smoking relative to baseline for circumstance $C$.

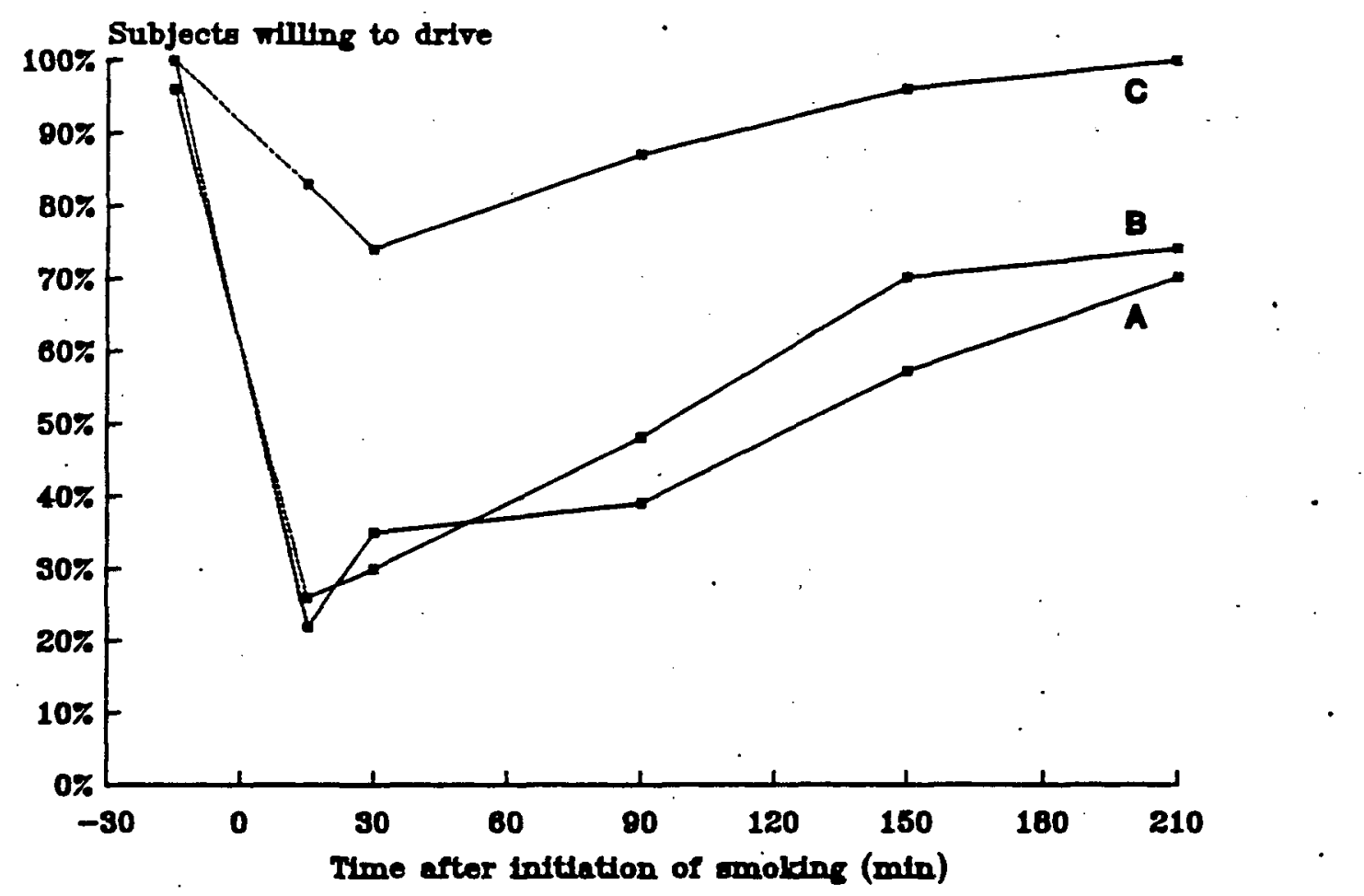

Figure 3.2 Percentage of subjects willing to drive under circumstances $A, B$ \& $C$ (see text) by Time.

\section{Perceived Alertness, Contentedness and Calmness}

Figure 3.3 shows subjects' ratings of perceived alertness, contentedness and calmness. Scores on these factors changed significantly over time $\left(F_{s, 17}=8.19,5.79 . \& 7.02 ; p<.001, .003 \&\right.$ .001 , respectively). Subjects felt significantly less alert relative to baseline until about $1 \frac{1}{2}$ hours after smoking $\left(\mathrm{F}_{1.22}=34.67,20.03 \& 16.97\right.$; all $p<.001 ; p_{c}=.01, .013 \&$ \& 017$)$. Separate sequential comparisons of subjective feelings of contentedness provided the same results $\left(F_{1.22}=27.24,19.98 \& 9.99 ; p<.001, .001 \& .004 ; p_{c}=.01, .013 \& .017\right)$. Feelings of calmness followed a different profile over time. Separate sequential comparisons showed that no significant change occurred during the first few hours after smoking, but at the end of the session subjects felt calmer than they had at baseline $\left(\mathrm{F}_{1.22}=12.25 ; p<.002 ; p_{c}=.01\right)$.

\section{Critical Tracking Test}

The average frequency at which the subjects lost control $\left(\lambda_{c}\right)$ was $4.40 \mathrm{rad} / \mathrm{s}$ before smoking and fell to $4.15 \mathrm{rad} / \mathrm{s}$ in the first test after smoking and gradually rose to baseline level in later tests. MANOVA however failed to show a significant Time effect.

$$
-24-
$$




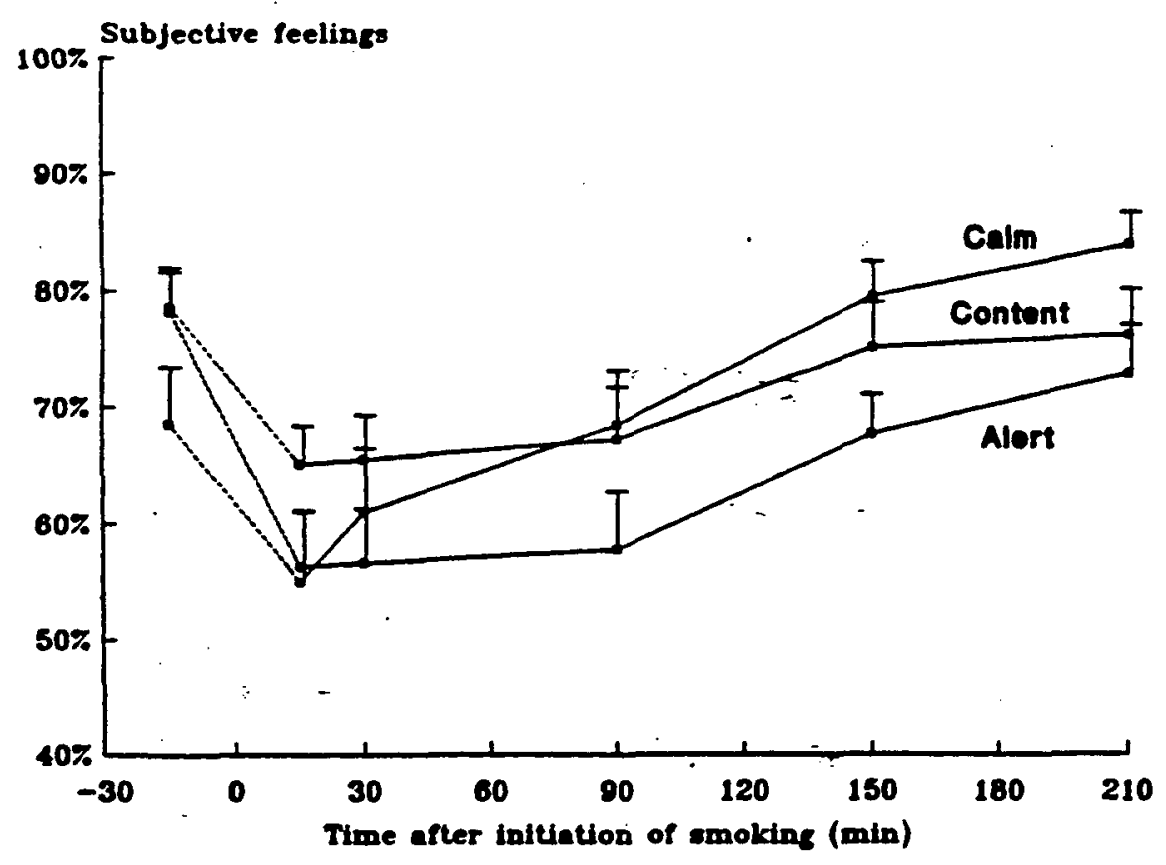

Figure 3.3 Mean (+SE) perceived alertness, contentedness and calmness by Time.

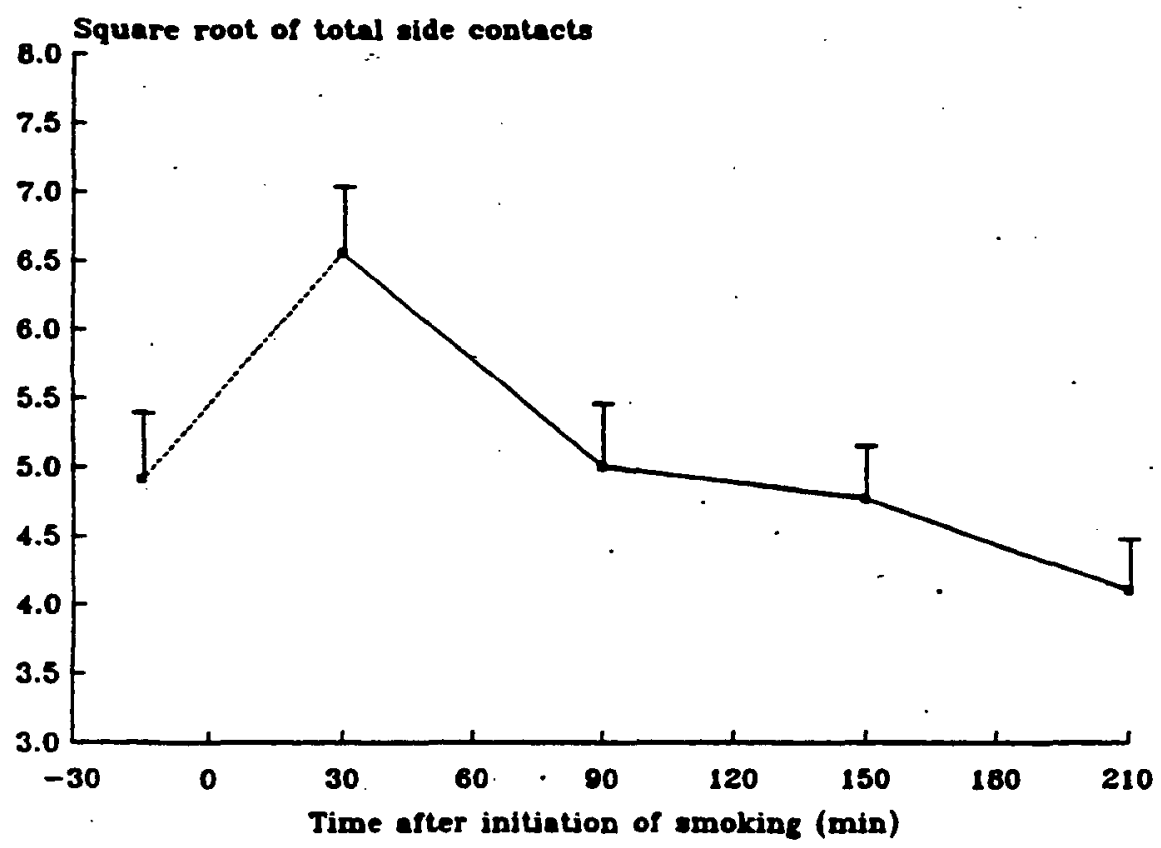

Figure 3.4 Mean (+SE) square root of total number of side contacts in the hand steadiness test by Time. 


\section{Hand Steadiness Test}

Figure 3.4 demonstrates that subjects' hand steadiness diminișed after smoking marijuana, but this effect dissipated rapidly after the first test. On the average, the subjects' hand steadiness was even superior at a time two hours after smoking to what it had been initially.

MANOVA revealed a significant Time effect $\left(\mathrm{F}_{4,18}=6.38 ; p<.002\right)$. Yet separate comparisons showed that hand steadiness was only significantly different from baseline in the first test after smoking $\left(\mathrm{F}_{1,22}=16.89 ; p<.001 ; p_{c}=.013\right)$.

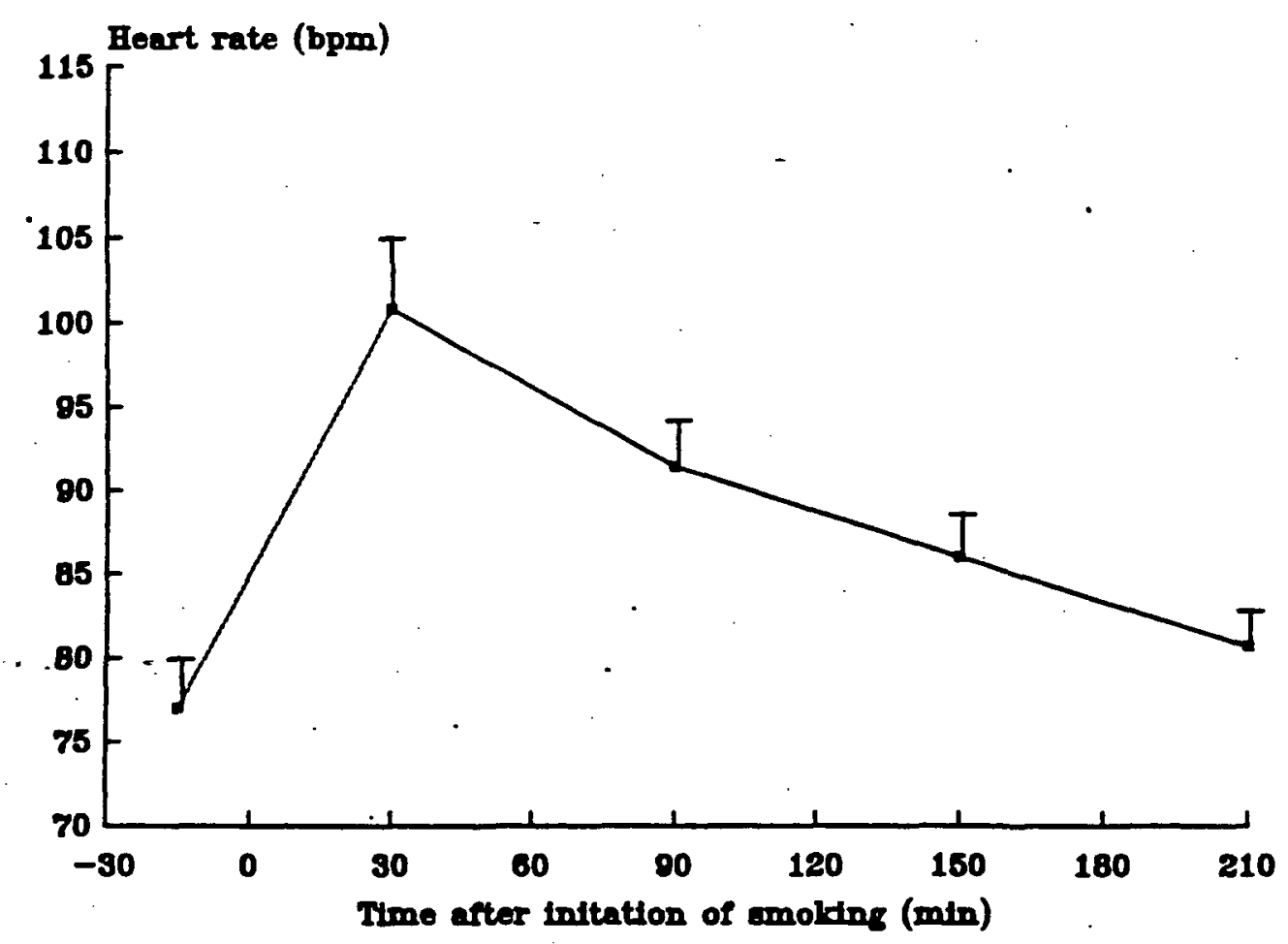

Figure 3.5 Mean (+SE) heart rate by Time.

\section{Heart Rate}

The subjects' average heart rate, presented in Figure 3.5, varied between 75 and 100 beats per minute (bpm) in close accordance with their subjective feelings. Thirty minutes after smoking, the increase in heart rate varied from 2 to $77 \mathrm{bpm}$, with a mean elevation of $24 \mathrm{bpm}$. Heart rate diminished over successive measurements approaching baseline values after $3 \frac{1 / 4}{4}$ hours. MANOVA revealed a significant Time effect $\left(\mathrm{F}_{4,18}=10.43 ; p<.001\right)$ and separate ANOVA comparisons showed that these elevations were significantly different from baseline at each but the last measurement $\left(\mathrm{F}_{1.22}=35.64,21.90,12.67 \& 2.37 ; p<.001, .001, .002 \& .14 ; p_{c}=.013\right.$, $.017, .025 \& .05$, respectively).

\section{Intra-Subject Relations between Variables}

Table 3.5 shows the average intra-subject correlations between [THC], $\log _{10}[\mathrm{THC}$, and [THC-COOH] with each of the other variables. The log transformation was again applied to 
achieve a more linear and homeoscadastic relationship between THC values and the other variables. The constant of 1 was added to the raw [THC] values for avoiding negative log transformations. It can be concluded from this table that, within subjects, higher plasma levels of the drug were associated with, and probably produced, increased heart rate, increased feelings of perceived "high", diminished hand steadiness, and diminished feelings of calmness, contentedness and alertness. The correlation with chitical tracking performance was not significant, confirming the insensitivity of the test. The log transformation of [THC] did not change the size of the correlations dramatically.

Table 3.5 Average intra-subject correlations $\left(* p<.05^{* *} p<.01 ; 2\right.$-tailed).

\begin{tabular}{lccc}
\hline & {$[\mathrm{THC}]$} & $\log _{10}([\mathrm{THC}]+1)$ & {$[\mathrm{THC}-\mathrm{COOH}]$} \\
\hline Heart Rate & $.79^{* *}$ & $.82^{* *}$ & $.68^{* *}$ \\
Perceived "high" & $.88^{* *}$ & $.95^{\circ *}$ & $.87^{* *}$ \\
$\lambda_{c}$ & -.20 & -.22 & -.27 \\
Side Contacts & $.48^{\circ}$ & $.45^{\circ}$ & .39 \\
Calmness & $-.51^{\circ}$ & -.38 & .02 \\
Contentedness & $-.57^{* *}$ &. $.59^{* *}$ & $-.46^{\circ}$ \\
Alertness & $-.64^{* *}$ & $-.72^{* *}$ & $-.66^{* *}$ \\
\hline
\end{tabular}

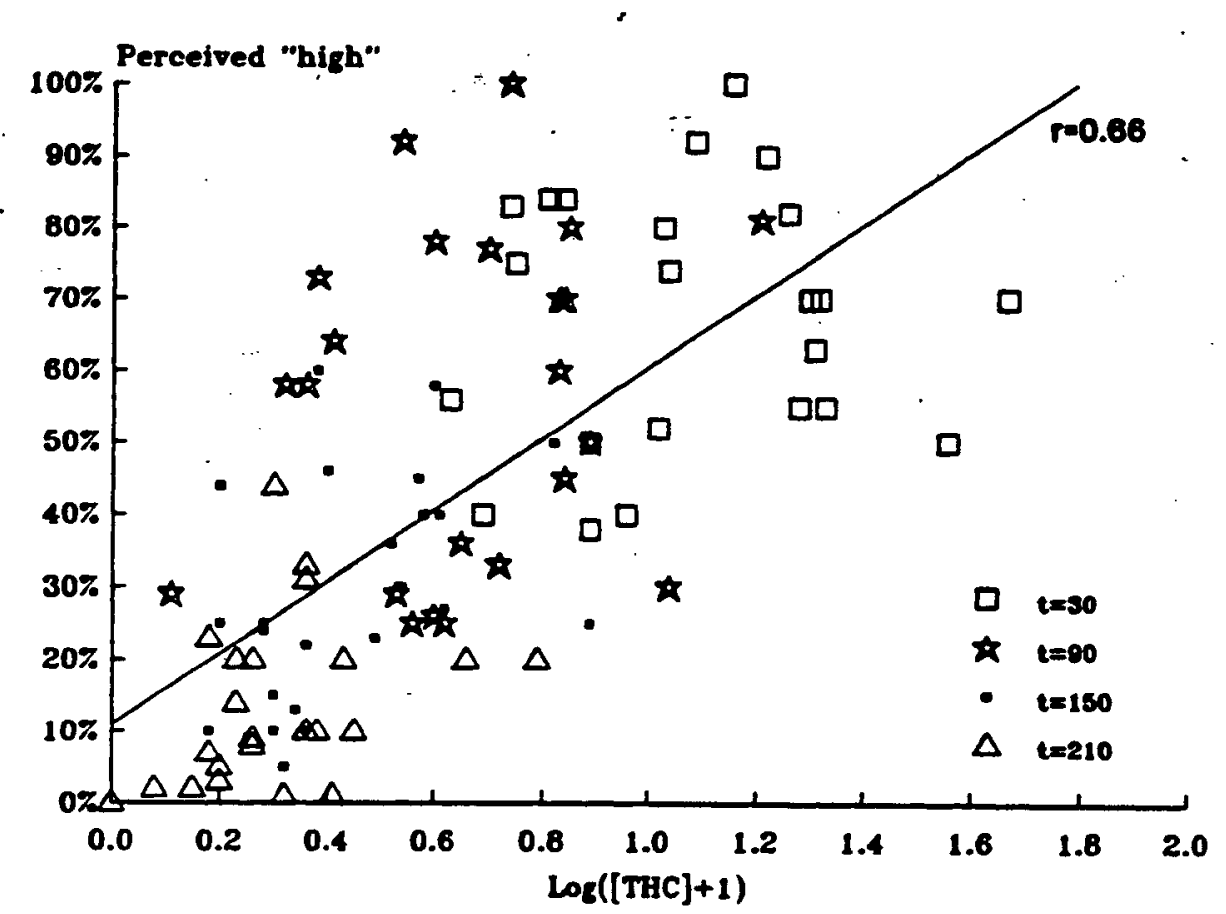

Figure 3.6 Scatter diagram of $\log ([\mathrm{THC}]+1)$ in plasma and perceived "high". Regression line comprising all data is inserted. 
Inter-Subject Relations between Variables

Inter-subject correlations between plasma concentrations of the drug and measures of subjective feelings, heart rate and performance were typically low ${ }^{-}(r<.50)$ at each sampling time. The correlations between $\log$ [THC] +1 and perceived "high", for example, were $r=0.07$ (ns), 0.19 (ns), $0.43(p<.05)$ and 0.35 (ns), at $t=40,100,160$ and 220 minutes after initiation of smoking, respectively. These low correlations are probably due to "restriction of range ", i.e. the variability in both measures is relatively small at each sampling time. If all available data $(\mathrm{N}=92,4$ measurements of 23 subjects) were included in the correlational computation the highest correlation found was that between $\log [\mathrm{THC}]+1$ and perceived "high" $(r=0.66$; Figure 3.6). In fact, this correlation is a combination of intra-subject and inter-subject correlations, and should therefore only be regarded as an indication of what the correlation might have been if all these data had been independent observations.

\section{DISCUSSION}

The pilot study's major purpose was to determine the amount of THC recreational users of cannabis smoke to reach a comfortable "high" and to establish from these results the maximum dose for subsequent driving studies. Median and mean amount of THC consumed were 308 and $292 \mu \mathrm{g} / \mathrm{kg}$ respectively. From these results it was decided that the maximum dose for subsequent driving studies would be $300 \mu \mathrm{g} / \mathrm{kg}$. This is considerably higher than doses that have usually been administered to subjects in experimental studies (typical, 100-200 $\mu \mathrm{g} / \mathrm{kg}$ THC). This could mean either that previous studies' THC doses were lower than those usually consumed by current users, or that the present study's subjects were less efficient smokers and, consequently, smoked more to achieve the same effect. Yet one important point should be raised. Imposed smoking procedures differ considerably between studies on marijuana's effects and can be divided at first glance in two types, self-paced in which subjects may smoke in their customary fashion and machine-paced procedures in which subjects smoke in a prescribed manner with regards to duration of inhalation of smoke and air, holding inhalation, etc. The former method is more realistic while the latter is more controlled. This study applied the former because of the disadvantage of the latter that it might induce unrealistic reactions in some subjects.

One way to contemplate this study's results is to compare the mean [THC] with those found in other studies in which similar procedures were applied with respect to smoking (ad lib fashion) and plasma analysis (GC/MS using a deuterated internal standard) as we did. To the authors' knowledge only one such study has been reported, namely by Ohlsson et al. (1980). Marijuana cigarettes containing $19 \mathrm{mg}$ THC were administered to eleven male subjects who were instructed to smoke in their own fashion such as to obtain the maximum desired "high". Subsequent gravimetric estimation showed that a mean of $13.0 \mathrm{mg}$ THC was consumed. [THC] values ranged between 5.4 and 18.0 (mean 12.4 ) $\mathrm{ng} / \mathrm{ml} 30$ minutes after termination of smoking. Only the males of the present study should be included in the comparison with Ohlsson's study, because of the significant difference found between [THC] values of both sexes in the current study. Eleven males in the present study smoked $22.3 \mathrm{mg}$ THC, on average, and their plasma concentrations ranged between 6.7 and 45.9 (mean 17.7 ) $\mathrm{ng} / \mathrm{ml} 40$ minutes after initiation of smoking. Thus, our subjects smoked $70 \%$ more THC than Ohlsson's subjects did, resulting in a $43 \%$ higher THC level in plasma, as measured 30 minutes after smoking. The average [THC] found in the present study was therefore in the expected direction. This observation may lead 
to the tentative conclusion that the preferred THC dose to reach a desired "high" in the present study was not due to inefficient smoking but to the fact that current marijuana (or hashish) users do smoke higher THC doses than previously administered in scientific studies.

Other results from the present study showed that perceived "high" and heart rate are very sensitive (psychological and physiological) measures of marijuana intoxication which confirms prior research. Impairments in laboratory tests performance were measured at the time of peak subjective feelings but generally, objective impairment dissipated more rapidly than the feelings themselves. All objective signs of impaired functions were gone within 1.5 hours after smoking. The explanation may be that practice and habituation effects, or both, occurred during the session concealing marijuana's impairment, or, that procedural errors have been made in administering these tests. No definitive answer can be provided and no conclusions can be drawn from this study with respect to marijuana's effects upon performance because of the lack of a control group. The study was simply not designed to estimate these effects, only to indicate whether either of these measures should be considered for inclusion in later studies. If any measure appeared to be systematically related to the inferred changes in THC's pharmacological activity over time, this measure might assume a high degree of practical importance. Correlational analyses, however, showed no strong relationships between [THC] or [THC-COOH] and performance in any test.

An important issue relating to traffic safety is whether subjects would drive a car while under the influence of marijuana. Although all subjects had admitted driving a car while intoxicated at least once before, a majority (about $65 \%$ ) of the subjects was not willing to drive a car for relatively unimportant reasons shortly after smoking when experiencing the drug's peak subjective reaction. However, most said they would drive, for a very urgent reason. On one hand, this means that the majority of the subjects are aware of a potential marijuana related driving impairment; on the other, a sizable minority $(35 \%)$ would not refrain from driving a car for unimportant reasons when they are experiencing a "high". These subjects in particular are a source of concern with respect to traffic safety, if marijuana smoking indeed impairs driving performance. Two questions that arise from these contemplations were addressed by the succeeding driving studies; namely, 1 . does marijuana adversely affect driving performance, and 2 . is willingness to drive after marijuana smoking related to driving impairment? 


\section{CHAPTER 4 - MARIJUANA AND DRIVING ON A RESTRICTED HIGHWAY}

\section{INTRODUCTION}

As mentioned in Chapter 1, THC's effects on actual driving performance have been assessed in a relatively small number of studies and only once in the presence of other traffic. The effects of doses up to about $250 \mu \mathrm{g} / \mathrm{kg}$ were modest, if present at all. These findings provided some assurance that it would be safe for subjects to undertake carefully supervised driving tests on normal roads and in traffic, even after treatment with the somewhat higher average dose (i.e. $300 \mu \mathrm{g} / \mathrm{kg}$ ) that was preferred by regular marijuana users in the pilot study. Nonetheless normal prudence demanded a demonstration of the test's safety in an environment resembling reality but where neither the subjects nor other road users would be endangered if the optimistic forecast proved false. One objective of the present study was to provide that demonstration using a standard test on a highway closed to other traffic.

The second objective was to define the dose-effect relationship between inhaled THC dose and that parameter of vehicular control which is measured in the standard test; i.e. standard deviation of lateral position (SDLP), an index of "weaving" amplitude the subject allows while attempting to maintain a constant speed and steady lateral position between traffic lane boundaries during uninterrupted highway driving. Alcohol's effects on SDLP were previously measured by Louwerens et al. $(1985,1987)$ in practically the same manner as THC's in this study. The earlier results showing a nearly perfect $(r=0.99)$ exponential relationship between mean blood alcohol concentration (0-0.12 g\%) and mean SDLP for 24 "social drinkers", serve admirably for evaluating THC's effects in the present case.

Other objectives were to measure changes in SDLP from tests after placebo to those following separate THC doses of 100,200 and $300 \mu \mathrm{g} / \mathrm{kg}$; and, to relate these objective measures of driving impairment to subjective impressions of driving quality and expressed willingness to drive in the same states of intoxication under normal circumstances.

\section{METHODS}

\section{Subjects}

The same twelve men and twelve women who participated in the pilot study served again as the subjects. Plasma from the pilot study was still not analyzed at the time data collection commenced. The male that apparently did not inhale marijuana smoke was therefore not dropped from the study. As before, his plasma samples showed neither THC nor THC-COOH. Data from this subject had to be again excluded from further analyses. Characteristics of the remaining 23 subjects are shown in the previous chapter (Table 3.1).

\section{Design, Doses and Administration}

Marijuana and placebo cigarettes were obtained from the same source as before. The subjects were treated on separate occasions with THC doses of $0,100,200,300 \mu \mathrm{g} / \mathrm{kg}$. Placebo 
cigarettes were prepared by ethanol extraction of THC from the plant stock. Marijuana cigarettes were prepared from batches containing $1.75 \%$ THC for the two lowest, and $2.57 \%$ THC for the highest dose. Cigarettes were cut to different lengths to provide the doses appropriate for the individuals' body. weights. It was necessary to provide the five largest subjects with two cigarettes at a time since one would not contain the total dose. Cigarettes were smoked through a plastic holder in a fashion determined by the subject but with the constraint that smoking had to be finished within ten minutes. After cessation of smoking, cigarettes were retained for subsequent gravimetric estimation of THC consumed. These analyses revealed that the average ( $\pm S D$ ) amount of consumed THC in the three marijuana conditions was $6.8( \pm 0.9), 13.6$ $( \pm 1.9)$ and $20.4( \pm 2.8) \mathrm{mg}$, which equals $94( \pm 4), 186( \pm 13)$ and $282( \pm 18) \mu \mathrm{g} / \mathrm{kg}$, respectively, or about $6 \%$ less than target doses. Order of treatments were counterbalanced. They were administered subject- and observer-blind (i.e. the investigator who prepared the treatments was not involved in their administration or with data collection).

\section{Testing Procedures}

Eight subjects were tested per night and all 24 within a week. Subjects were tested at the same times and on the same days of the week for four consecutive weeks. Breath and urine tests were executed upon the subjects' arrival to check for the presence of alcohol and illicit drugs. If cannabinoids were found in the urine, a blood sample was taken for later verification of the presence of THC. Two subjects commenced smoking at a time at $t=0$ (Table 4.1). Driving tests were performed twice, beginning at $t=40$ and 100 minutes and lasting 15-20 minutes. Blood samples were taken before the driving tests. The subjects' pulse was taken and their performance measured in two laboratory tests that began after the driving tests. Subjective assessments were made immediately after smoking, and before and after the driving tests. Before the start of the experiment, subjects were individually trained to operate the vehicle under generally the same conditions as the tests later occurred.

Table 4.1 Schedule of activities on test-days.

\begin{tabular}{|c|c|}
\hline Relative Time ( $\mathrm{min}$ ) & Activity \\
\hline $\begin{array}{r}0-10 \\
30-35 \\
40-60 \\
70-80\end{array}$ & $\begin{array}{l}\text { Smoking } \\
\text { Blood Sampling } \\
\text { Standard Driving Test } \\
\text { Tracking and Hand Steadiness Tests } \\
\text { Heart rate and Blood Pressure }\end{array}$ \\
\hline $\begin{array}{r}90-95 \\
100-120 \\
130-140\end{array}$ & $\begin{array}{l}\text { Blood Sampling } \\
\text { Standard Driving Test } \\
\text { Tracking and Hand Steadiness Tests } \\
\text { Heart rate and Blood Pressure }\end{array}$ \\
\hline
\end{tabular}

\section{Driving Test}

The driving test, developed and standardized by O'Hanlon et al. $(1982,1986)$ and applied in more than $\mathbf{4 0}$ open- and closed-road studies by three Dutch Institutes during the last decade, 
measures the ability to control an instrumented vehicle's speed and lateral position. Subjects were instructed to maintain speed at $90 \mathrm{~km} / \mathrm{h}(56 \mathrm{mph})$, or less if they felt incapable of driving safely at that speed, and a steady lateral position between the delineated boundaries of the traffic lane.

Driving was performed over a $11 \mathrm{~km}(6.8 \mathrm{mi})$ section of a primary highway (A76) that connects the Dutch cities of Geleen and Heerlen. Two lanes in the same direction were closed to normal traffic between the hours of 19.00 and 24.00 on three consecutive week-nights over four consecutive weeks of testing. Driving began at one end of the section, involved turning at the other and ended with a return to the origin. A licensed driving instructor accompanied each subject. He was charged with responsibility for ensuring safety at all times and was able to intervene, if necessary, using redundant vehicular controls.

Two Volvo station wagons containing essentially the same instrumentation were employed in the study. The first of a pair of subjects who received treatments together departed from the origin driving one vehicle and was followed by the second driving the other after $21 / 2$ minutes. The first subject waited for the arrival of the second at the turning point before returning to the origin. The purpose was to avoid having the subjects, travelling in opposite directions, meet en route. The major instrumentation comprised devices for acquiring continuous analog signals representing steering wheel angle, vehicle-speed and lateral position relative to the midline stripe delineation, and a computer system for recording those signals continuously at a $4 \mathrm{~Hz}$ sampling rate.

The primary dependent variable was the standard deviation of lateral position (SDLP), which has been shown to be both highly reliable (typical test-retest correlation of 0.7-0.9) and very sensitive to the influence of sedative drugs and alcohol. Other dependent variables were mean speed (SP) and standard deviation of speed (SDSP) and steering wheel angle (SDST).

\section{Questionnaires}

The same subjective questionnaires used in the pilot study were administered to the subjects immediately after cessation of smoking $(t=10)$ and again at the beginning of each driving test $(t=40 \& 100)$. At the end of each driving test $(t=60 \& 120)$, the subjects were required to retrospectively rate their effort made while performing the test (Zijlstra and Van Doorn, 1985; Meyman and Zijistra, 1986) and subjective driving quality on respective visual-analog scales. Scores on these scales will be expressed as percentage of total scale and percentage of "normal" driving quality, respectively. Questionnaires are enclosed in Appendix A.

\section{Laboratory Tests}

Two of the tests employed in the pilot study were also applied here, namely the critical tracking and hand steadiness tests. Exactly the same procedures were employed in their administration as described in the previous chapter.

\section{Physiological Assessments}

Heart rate, systolic and diastolic blood pressure were measured by means of a digital blood pressure monitor prior to the hand steadiness test. 
Blood Sampling

Blood samples were taken by venepuncture. The samples ( 2 aliquots containing $10 \mathrm{ml}$ each) were heparinized and centrifuged. The plasma fractions were placed in frozen $\left(-20^{\circ} \mathrm{C}\right)$ storage prior to analysis for [THC] and [THC-COOH].

\section{Data Analysis}

All data measured on ratio or interval scales were taken in a mixed between-groups, withinsubjects MANOVA analysis. Sex was the between-groups factor. Dose (4 levels) and Time after dosing $(2$ or 3 levels) were factors tested within-subjects. If a significant $(p<.05)$ Dose effect was found, repeated measures ANOVAs were conducted for testing differences between measures obtained after placebo and each THC dose, separately. Data in these cases were collapsed across Sex and Time unless the MANOVA analysis had revealed a significant interaction between either factor and Dose. In the figures, the mean of the variable is depicted by the height of the bar and its standard error (SE) by the height of the vertical line above the bar.

Separate dose effects were tested using the "Sequential Bonferroni" procedure for adjusting the $\alpha$-probability criterion $\left(p_{c}\right)$ in accordance with the number of separate comparisons in a given set (Overall and Rhoades, 1987). This means that for the largest of three differences tested at once, $p$ had to be less than $.05 / 3=.017$ to be judged significant. For the second, it had to be $<.05 / 2=.025$; and for the smallest difference, $p<.05$. The adjustment had the effect of holding the probability of making a type I error at $p \leq .05$ over the entire set of comparisons.

The subjects' expressions of willingness to drive were made on the basis of a dichotomous decision and could not for that reason be analyzed in the same manner as other variables. These data were therefore analyzed using Cochran's Q-statistic test for assessing differences between dosing conditions at each time of testing, separately.

Significant Sex effects were generally absent. Results pertaining to differences between the sexes are therefore only reported for the exceptional cases where the differences were significant.

Inter-subject and intra-subject correlations were computed and tested as described in the previous chapter.

\section{RESULTS}

\section{Plasma Concentrations of the Drug}

Though consumed dose differed little between subjects, [THC] and [THC-COOH] varied enormously. Thirty minutes after smoking $300 \mu \mathrm{g} / \mathrm{kg}$, for example, [THC] ranged between 1.6 and $59.6 \mathrm{ng} / \mathrm{ml}$. Table 4.2 shows mean, median and range of [THC] and [THC-COOH] by Dose and Time. Placebo values were not used in the statistical analyses since these were zero in most cases.

As shown by Table 4.2 plasma concentrations of the drug were clearly related to the administered dose and time of blood sampling. MANOVA confirmed this observation yielding a significant Dose $\left(\mathrm{F}_{2,20}=14.65 \& 16.59\right.$ for [THC] and [THC-COOH], respectively; both $p<.001)$ and Time $\left(\mathrm{F}_{1.21}=50.76 \& 21.16\right.$; both $\left.p<.001\right)$ effect. There was a significant Dose by Time interaction for [THC] $\left(\mathrm{F}_{2.20}=10.07 ; p<.001\right)$ and not [THC-COOH]. Though not shown 
in the table, males had significantly higher [THC-COOH] values, $9 \mathrm{ng} / \mathrm{ml}$ on average, than females $\left(F_{1,21}=4.49 ; p<.05\right)$; average $[\mathrm{THC}]$ values were virtually the same for both sexes.

Table 4.2 Mean, median and range of [THC] and [THC-COOH] in $\mathrm{ng} / \mathrm{ml}(\mathrm{N}=23)$.

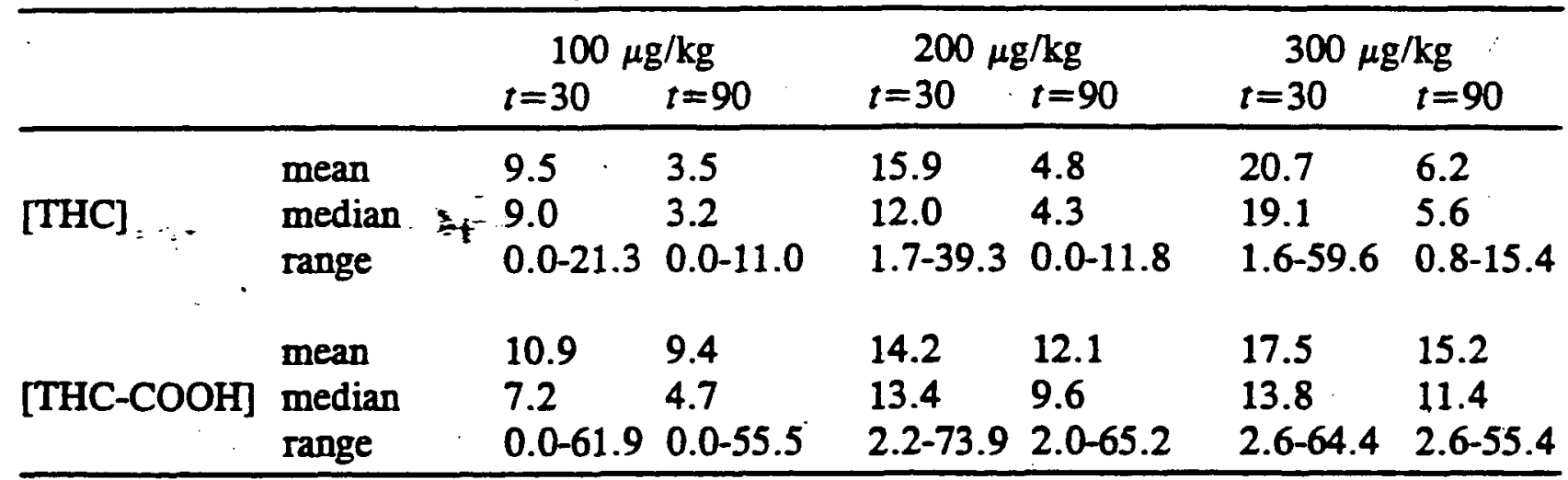

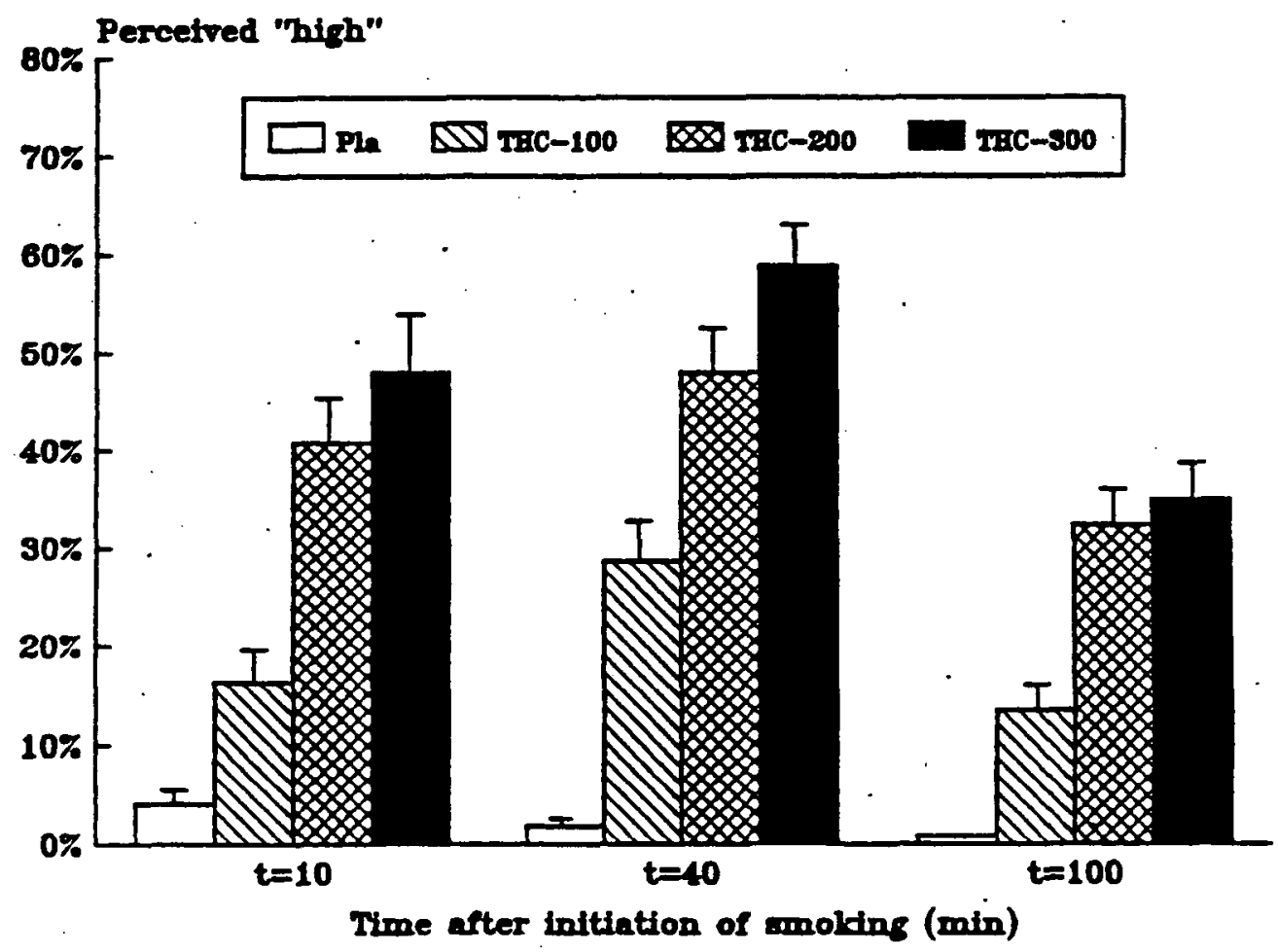

Figure 4.1 Mean (+SE) perceived "high" by Dose and Time.

\section{Perceived "high"}

Average subjective feelings of intoxication ("high") were dose-related and highest just before the first driving test (Figure 4.1). Relative to maximum personal experience, peak levels of intoxication were about $30 \%, 50 \%$ and $60 \%$ after 100,200 and $300 \mu \mathrm{g} / \mathrm{kg}$ doses, respectively. 
Feelings of "high" after placebo were near zero in most cases. Therefore changes occurring after marijuana smoking, relative to placebo, instead of the raw values, were analyzed by MANOVA. Change scores were significantly different from zero $\left(\mathrm{F}_{1,21}=125.22 ; p<.001\right)$, dose-related $\left(F_{2.20}=21.76 ; p<.001\right)$ and time-related $\left(F_{2,20}=36.68 ; p<.001\right)$. Females felt more intoxicated than males $\left(F_{1,21}=4.59 ; p<.05\right)$, but there was no significant Sex by Dose interaction. Univariate analyses revealed that intoxication ratings were different from zero and followed a quadratic trend in all marijuana conditions.

\section{Driving Performance}

No driving tests had to be stopped for safety reasons by the driving instructols. Yet in two instances, both after the highest THC dose; the instructor felt compelled to intervene. In one case, the driving instructor twice warned the subject to avoid a screwdriver laying on the road $=$ but when he failed to react the instructor did by steering away from the object (it was immediately removed following this incident). The subject was queried about this situation after termination of the ride. He recalled that the driving instructor had taken control but did not recall why! In the other case, a subject failed to decelerate as he approached the turning point. The instructor told him to do so whereupon the subject abruptly brought the vehicle to.a stop using the break.

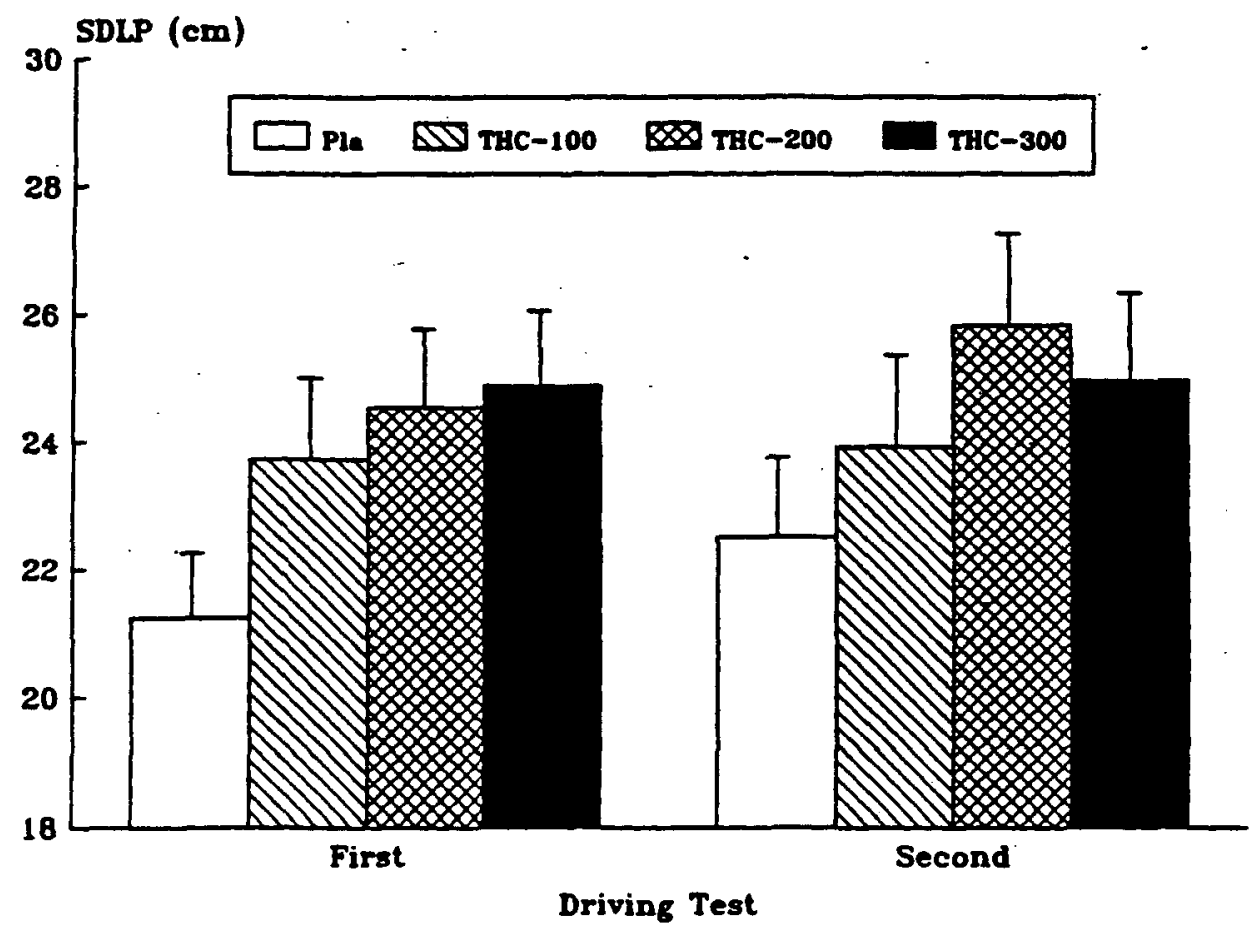

Figure 4.2 Mean (+SE) standard deviation of lateral position by Dose and Time.

SDLP reliability was high in this study: the correlation between measurements made on successive trials after placebo was $0.92(p<.001)$. Correlations betiveen SDLP values on the 
first and second trials after THC doses of 100,200 and $300 \mu \mathrm{g} / \mathrm{kg}$ were $0.90,0.91$ and 0.91 (all $p<.001$ ), respectively. This means that subjects' SDLPs from the second ride were almost perfectly related to those of the first, and, consequently, that SDLP measurement error was very small.

Mean values of SDLP are shown in Figure 4.2 as a function of Dose and Time. Higher values, indicating poorer road tracking performance, were found after marijuana smoking. MANOVA revealed that the $\operatorname{Sex}$ effect $\left(\mathrm{F}_{1.21}=4.41 ; p<.05\right)$ was significant, females having higher SDLPs than males. The Sex by Dose interaction, however, was not significant $\left(\mathrm{F}_{3.19}=2.54 ; p<.09\right)$, indicating that the effect of marijuana was grossly the same for both sexes. The analysis indicated a clear Dose effect $\left(F_{3.19}=8.92 ; p<.001\right)$. Neither Time nor Dose by Time effects were statistically significant indicating that impairment after marijuana was the same in both trials. Separate dose comparisons revealed that performance after marijuana was always worse than after placebo $\left(F_{1.22}=7.45,19.96 \& 14.58\right.$ for the $100,200 \& 300 \mu \mathrm{g} / \mathrm{kg}$ conditions, respectively; $\left.p<.012 ; .001 \& .001 ; p_{c}=.05, .017 \& .025\right)$.

Mean speed was vêry close to that established as the target by instructions and varied between conditions from 88.9 to $90.5 \mathrm{~km} / \mathrm{h}$ ( 55.3 to $56.2 \mathrm{mph}$ ). Subjects drove $0.4 \mathrm{~km} / \mathrm{h}$ $(0.25 \mathrm{mph})$ faster after the $100 \mu \mathrm{g} / \mathrm{kg}$ dose, and $0.4 \mathrm{~km} / \mathrm{h}$ slower after both of the higher doses, than after placebo. Yet these differences were small as percentages of the average, and not significant. On average, speed was $0.6 \mathrm{~km} / \mathrm{h}(0.37 \mathrm{mph})$ higher in the second than in the first ride resulting in a significant Time effect $\left(\mathrm{F}_{1,21}=5.24 ; p<.04\right)$.

Differences in standard deviation of speed were greater between sexes than between conditions. There was a significant $S e x$ effect $\left(F_{1,21}=6.99 ; p<.02\right)$, females having greater difficulty in maintaining a constant speed than males. SDSP was, after each THC dose, higher in the first, but lower in the second ride relative to placebo. Changes from placebo were, however, small and not significant.

Changes in standard deviations of steering wheel angle were small and not significant.

\section{Perceived Driving Quality and Effort}

Figures 4.3 and 4.4 respectively show mean perceived driving quality, and effort to accomplish the test, by Dose and Time. Subjects rated their driving performance as better than "normal" after placebo. Ratings were around normal after the lowest THC dose but poorer than normal to about the same degrees after both of the higher doses. Differences attributable to Dose were significant $\left(\mathrm{F}_{3.19}=3.76 ; p<.03\right)$. Separate dose comparisons showed that the effects of all three doses were significantly different from placebo $\left(F_{1.22}=4.80,8.64 \& 10.76\right.$ for the 100,200 and $300 \mu \mathrm{g} / \mathrm{kg}$ respectively; $\left.p<.04, .008 \& .003 ; p_{c}=.05, .025 \& .017\right)$. Perceived driving quality was higher in the second driving test than in the first $\left(\mathrm{F}_{1.21}=8.06 ; p<.01\right)$, but the lack of a significant Dose by Time interaction indicated that this was independent of the administered dose.

Effort to accomplish the driving test increased systematically with the administered dose. Although there were only small differences in either SDLP or perceived driving quality between the two highest dose conditions, effort was, especially in the first trial, greater after the $\mathbf{3 0 0}$ than after the $200 \mu \mathrm{g} / \mathrm{kg}$ dose. This suggests that subjects had to compensate more after the highest dose to achieve about the same objective and subjective driving performance. MANOVA revealed significant Dose $\left(\mathrm{F}_{3.19}=13.41 ; p<.001\right)$, Time $\left(\mathrm{F}_{1.21}=8.59 ; p<.008\right)$ and Dose by Time $\left(F_{3.19}=10.27 ; p<.001\right)$ effects. Separate dose comparisons showed that perceived effort was higher in all three marijuana conditions $\left(F_{1.22}=7.57,20.17 \& 37.96\right.$ for the 100,200 and 300 


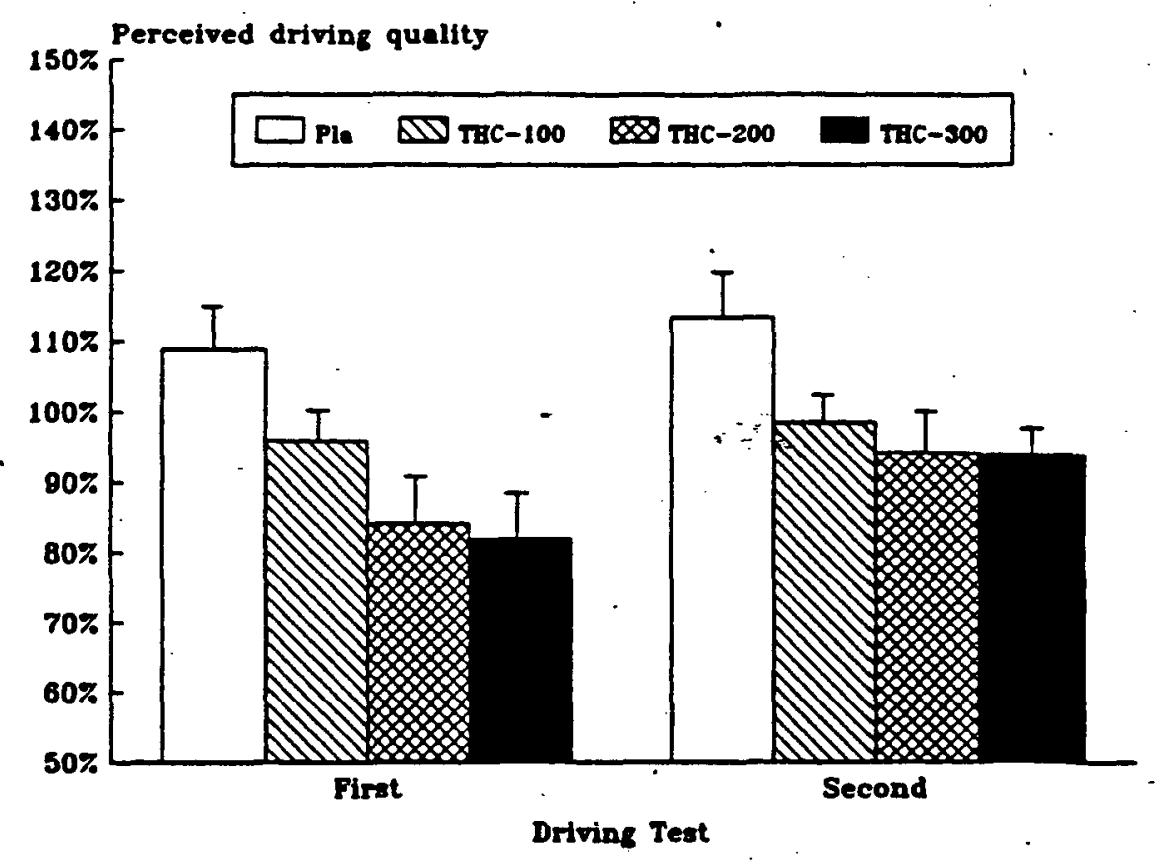

Figure 4.3 Mean (+SE) perceived driving quality, expressed as percentage of "normal", by Dose and Time.

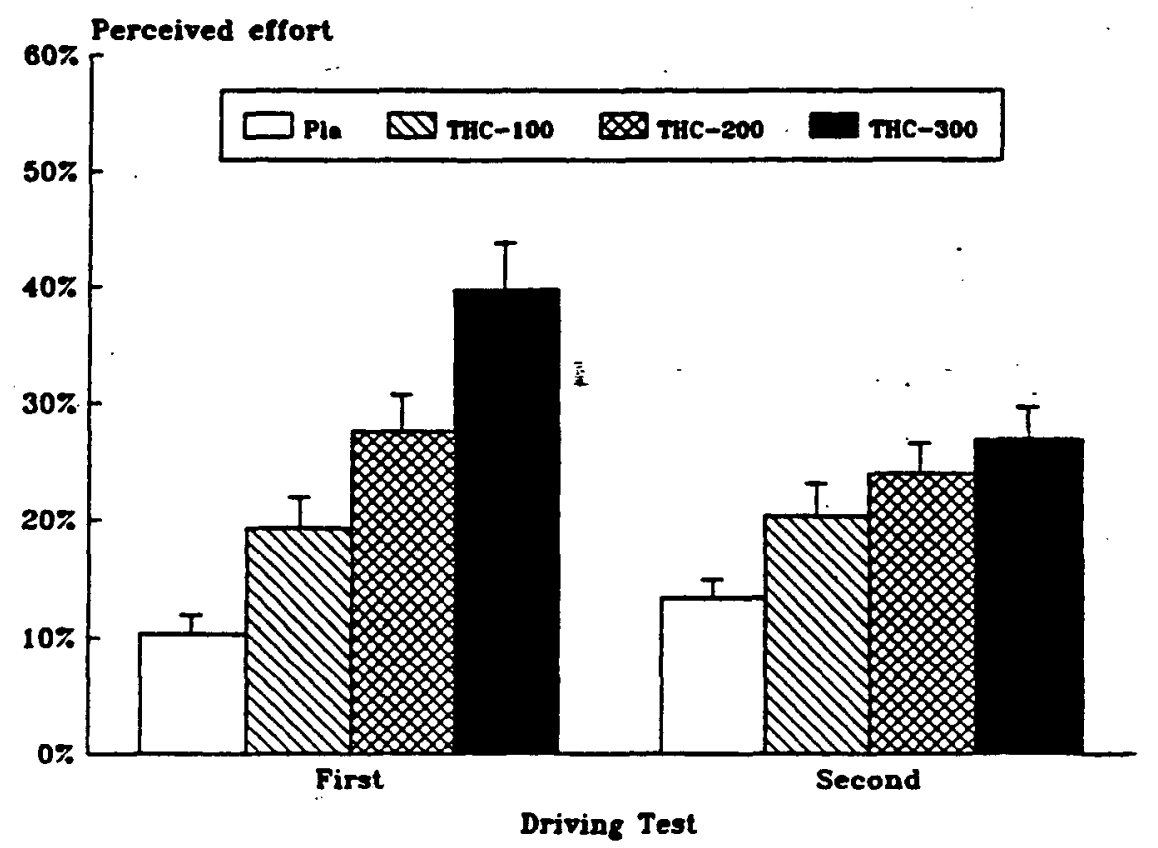

Figure 4.4 Mean (+SE) perceived effort to accomplish the test, expressed as percentage of maximum of scale, by Dose and Time. 
$\mu \mathrm{g} / \mathrm{kg}$, respectively; $\left.p<.012, .001 \& .001 ; p_{c}=.05, .025 \& .017\right)$ than in the placebo condition. The Dose by Time interaction was significant after the two higher doses $\left(F_{1.22}=8.45 \& 24.95\right.$ for the 200 and $300 \mu \mathrm{g} / \mathrm{kg}$, respectively; $p<.008 \& .001 ; p_{c}=.025 \& .017$ ); i.e. the effort requirement diminished over the interval separating smoking and driving in these conditions.

\section{Willingness to Drive}

Table 4.3 presents the percentage of subjects willing to drive under specified conditions of different urgencies ( $A$. unimportant though gratifying; $\boldsymbol{B}$. important but avoidable; and, $\boldsymbol{C}$. urgent).

Table 4.3 Percentage of subjects willing to drive under circumstances $A, B \& C$ (see text) by Dose and Time. Rightmost columns display Cochran $\mathrm{Q}$-statistic $(\mathrm{df}=3)$ with $p$ values.

\begin{tabular}{llllllll}
\hline & & $0 \mu \mathrm{g} / \mathrm{kg}$ & $100 \mu \mathrm{g} / \mathrm{kg}$ & $200 \mu \mathrm{g} / \mathrm{kg}$ & $300 \mu \mathrm{g} / \mathrm{kg}$ & \multicolumn{2}{l}{ Cochran's Q $p<$} \\
\hline & $t=10$ & 96 & 87 & 48 & 48 & 23.54 & .001 \\
A $t=40$ & 96 & 65 & 48 & 43 & 23.22 & .001 \\
& $t=100$ & 96 & 83 & 65 & 61 & 16.40 & .001 \\
& & & & & &. \\
& $t=10$ & 91 & 83 & 65 & 57 & 12.00 & .008 \\
B $t=40$ & 91 & 70 & 48 & 61 & 15.14 & .002 \\
& $t=100$ & 91 & 87 & 74 & 57 & 17.22 & .001 \\
& $\cdot$ & & & & & & \\
& $t=10$ & 100 & 96 & 91 & 74 & 13.11 & .005 \\
C $t=40$ & 100 & 96 & 87 & 74 & 11.45 & .01 \\
& $t=100$ & 100 & 96 & 96 & 87 & 6.33 & NS \\
\hline
\end{tabular}

Subjects' responses were similar to those in the pilot study. The lower the administered THC dose and the more urgent the reason for driving, the more subjects declared that they would be willing to drive. According to the subjects' declarations, 40-60\% would have driven for unimportant reasons shortly after the two highest doses. However, more than $75 \%$ would have driven for an urgent reason. Nearly all would have driven $1 \frac{1}{2}$ hours after smoking for an urgent reason when objectively measured driving performance was still impaired. Differences between treatments were significant in all cases, except one: the percentages of subjects who said they would have driven for an urgent reason 100 minutes after initiation of smoking were not different between treatment conditions.

\section{Perceived Alertness, Contentedness and Calmness}

Subjective feelings of alertness, contentedness and calmness were all affected by Dose $\left(\mathrm{F}_{3.19}=11.18,4.86 \& 5.14\right.$, respectively; $\left.p<.001, .011 \& .009\right)$. After marijuana smoking, subjects felt less alert, content and calm. Significant Time effects were found for alertness and calmness $\left(F_{2,20}=7.89 \& 8.10\right.$, respectively; both $\left.p<.003\right)$ : subjects felt more alert and calm later 
in the session. A significant Sex by Time effect was found for feelings of contentedness, females feeling less and males more content later in the session. Separate comparisons showed that all three THC doses produced significantly. reduced feelings of alertness $\left(F_{1.22}=12.46,28.94 \&\right.$ 24.80 for the 100,200 and $300 \mu \mathrm{g} / \mathrm{kg}$, respectively; $p<.002, .001 \& .001 ; p_{c}=.05, .025 \&$ $.017)$. Only the two higher doses produced significantly reduced feelings of contentedness $\left(F_{1.22}=8.70 \& 15.05\right.$ for the 200 and $300 \mu \mathrm{g} / \mathrm{kg}$, respectively; $\left.p<.007 \& .001 ; p_{c}=.025 \& .017\right)$ and calmness $\left(F_{1.22}=14.29 \& 11.70\right.$ for the 200 and $300 \mu \mathrm{g} / \mathrm{kg}$, respectively; $p<.001 \& .002$; $p_{c}=.017 \&$.025).

\section{Critical Tracking Test}

Subjects' tracking performance, i.e. $\lambda_{r}$, was not affected by THC. Males performed significantly better than females $\left(F_{1.21}=12.61 ; p<.002\right)$ and performance of all subjects was worse at the second than at the first assessment $\left(F_{1,21}=10.89 ; p<.003\right)$, but these observations are not of great concern.

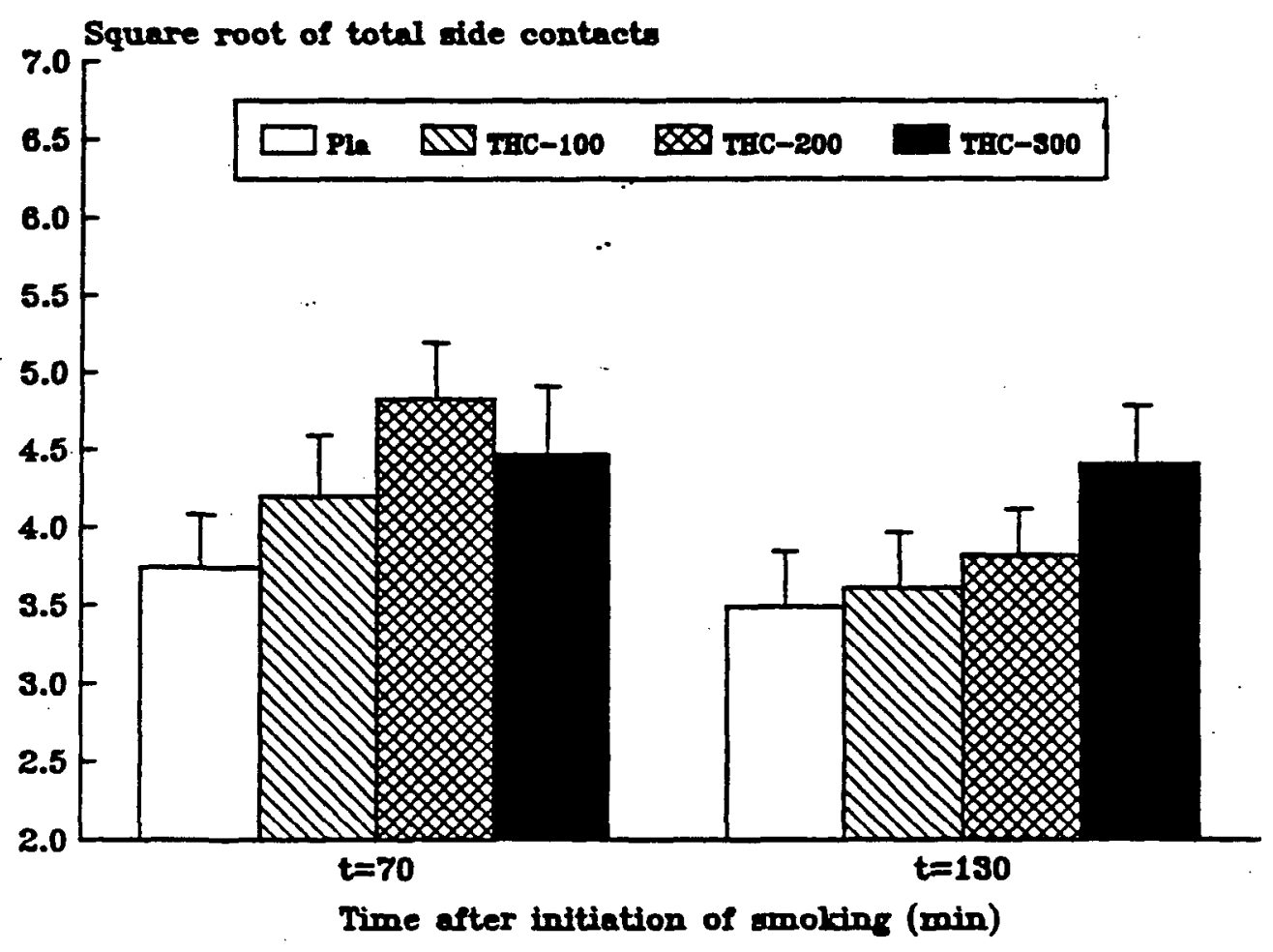

Figure 4.5 Mean (+SE) square root of total number of side contacts in the hand steadiness test by Dose and Time.

\section{Hand Steadiness Test}

Figure 4.5 demonstrates that hand steadiness diminished after all THC doses. MANOVA revealed a significant Dose $\left(\mathrm{F}_{3.19}=5.04 ; p<.01\right)$ and Time $\left(\mathrm{F}_{1.21}=8.61 ; p<.008\right)$, but no Dose by Time effect. This means that marijuana's impairment was still persistent two hours after 
smoking. Separate comparisons showed that both higher THC doses, but not the lowest, diminished hand steadiness $\left(F_{1.22}=7.67 \& 11.76\right.$ for the 200 and $300 \mu \mathrm{g} / \mathrm{kg}$, respectively; $\left.p<.011 \& .002 ; p_{c}=.025 \& .017\right)$.

\section{Heart Rate and Blood Pressure}

Heart rate and blood pressure were measured 70 and 130 minutes after the initiation of smoking. Yet as Figure 4.6 demonstrates, heart rate was stiii zlevated in a dose related manner at both assessments. MANOVA confirmed this observation revealing a significant Dose effect $\left(F_{3.19}=7.71 ; p<.001\right)$. Heart rate was always lower at the second assessment resulting in a significant Time effect $\left(F_{1,21}=24.34 ; p<.001\right)$. Dose by Time interaction was not significant. Separate comparisons revealed that all three THC doses produced significant heart rate elevations relative to placebo $\left(\mathrm{F}_{1,22}=9.00,13.62 \& 20.61\right.$ for the 100,200 and $300 \mu \mathrm{g} / \mathrm{kg}$, respectively; $\left.p<: 007, .001 \& .001 ; p_{c}=.05, .025 \& .017\right)$.

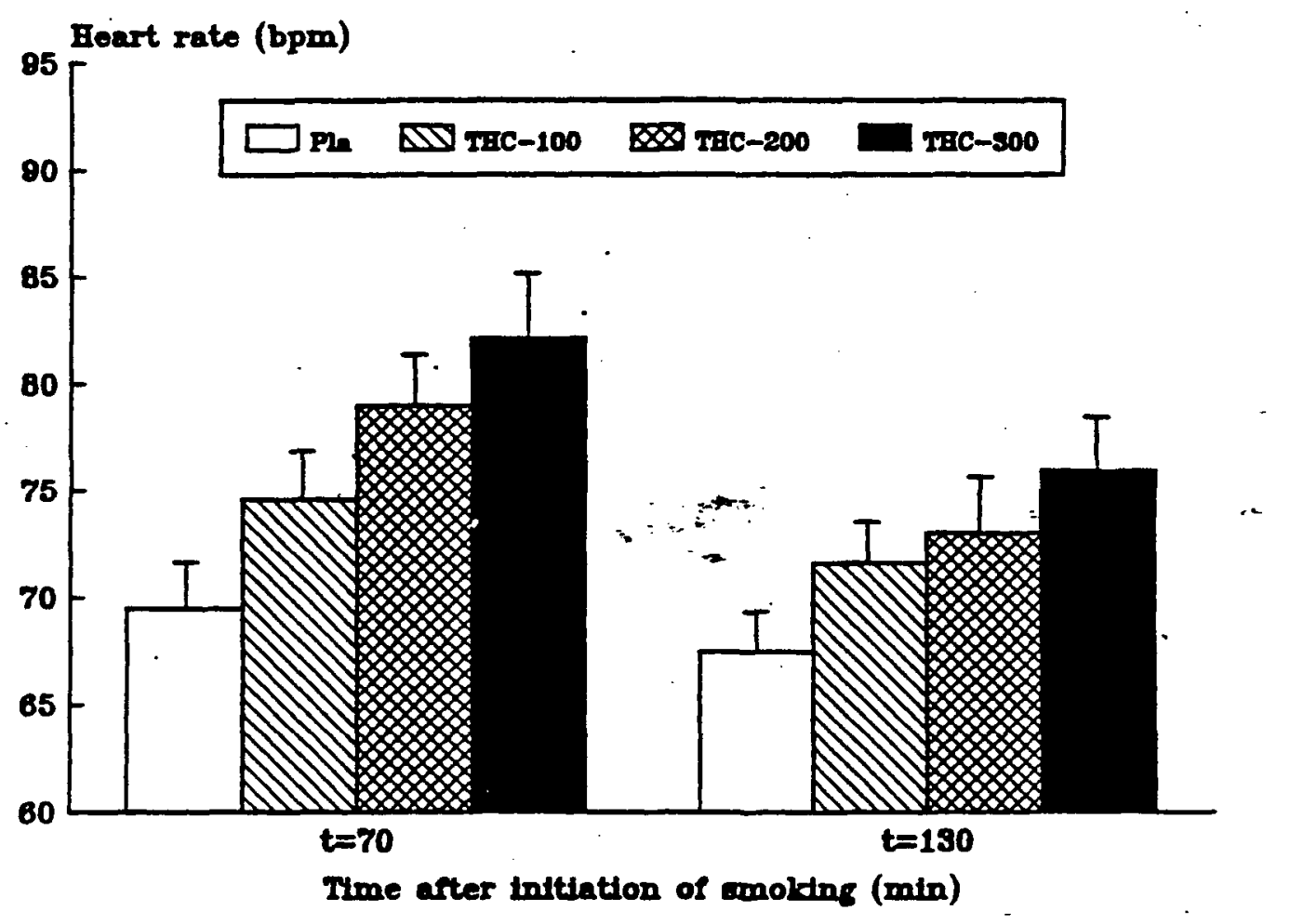

Figure 4.6 Mean (+SE) heart rate by Dose and Time.

Systolic and diastolic blood pressure measurements were simultaneously analyzed in one "doubly" repeated measures design. This means that both measures are analyzed in one multivariate design. Univariate effects of THC on systolic and diastolic pressures were only tested for significance, separately, if the multivariate Dose effect was significant. The lowest THC dose produced slightly lower blood pressure relative to placebo, whereas the highest dose had the opposite effect. Changes in blood pressures varied between -2.0 to $+5.8 \mathrm{mmhg}$. MANOVA failed, however, to reveal either a significant Dose or Dose by Time effect. The only 
significant factor was Time $\left(\mathrm{F}_{1.21}=6.92 ; p<.005\right)$, indicating that blood pressure decreased significantly from the first to the second assessment: this occurred in both the systolic $\left(\mathrm{F}_{1.21}=12.92 ; p<.002\right)$ and diastolic blood pressure $\left(\mathrm{F}_{1,21}=4.77 ; p<.05\right)$.

\section{Intra-Subject Relations between Variables}

Table 4.4 shows the average intra-subject correlations of [THC], [THC-COOH], and SDLP with each of the other variables. The averages were computed from 23 intra-subject correlations, calculated from data obtained at eight sampling times (twice in each condition). The table shows that higher plasma levels of the drug were associated with increased feelings of perceived "high", higher levels of effort to accomplish the driving test, decreased ratings of subjective driving quality, and increased heart rate. There was no strong relationship, within subjects, between plasma levels of the drug and SDLP. This was because drug concentrations declined between the first and second sampling time, whereas SDLP scores hardly changed. When

- average intra-subject correlations were computed for the first and second sampling times separately, the correlations between $[T H C]$ and $\operatorname{SDLP}$ were $0.59(p<.01)$ and $0.42(p<.05)$, respectively.

Table 4.4 Average intra-subject correlations $(* p<.05 * * p<.01 ; 2$-tailed).

\begin{tabular}{|c|c|c|c|}
\hline & [THC] & [THC-COOH] & SDLP \\
\hline Perceived "high" & $.83^{\circ *}$ & $.80^{* *}-(x-1)$ & .41 \\
\hline Perceived Effort & $.53^{* *}$ & $.58^{* *}$ & .30 \\
\hline Perceived Driving Quality & $-.43^{\circ}$ & $\therefore .44^{\circ}$ & -.29 \\
\hline SDLP & .23 & .35 & 1.00 \\
\hline SP & -.35 & -.34 & .05 \\
\hline SDSP & -.02 & .05 & .16 \\
\hline$\lambda_{c}$ & .11 & .07 & -.22 \\
\hline Side Contacts & .26 & .20 & .14 \\
\hline Heart Rate & $.49^{\circ}$ & .39 & .27 \\
\hline
\end{tabular}

\section{Inter-Subject Relations between Variables}

Relationship between Drug Levels and Performance: Inter-subject correlations between plasma concentrations of the drug and performance were calculated to determine whether subjects with higher plasma levels of the drug performed poorer than those that had lower plasma levels. Correlations between driving performance and performance in the laboratory were also calculated. The results are presented in Table 4.5. It appeared that correlations involving plasma concentrations were greater when logarithmic values of THC values were used in the computation $(1 \mathrm{ng} / \mathrm{ml}$ was added to all THC values before the transformation in order to avoid negative $\log$ values). Correlations with THC-COOH were generally smaller than those with log THC values; therefore only correlations with $\log$ THC values are shown in the table. 
Table 4.5 Inter-subject correlations between drug concentrations in plasma and raw performance scores in marijuana conditions $\left(* p<.05,{ }^{* *} p<.01 ; 2\right.$-tailed).

\begin{tabular}{|c|c|c|c|c|c|c|}
\hline & \multicolumn{2}{|c|}{$100 \mu \mathrm{g} / \mathrm{kg}$} & \multicolumn{2}{|c|}{$200 \mu \mathrm{g} / \mathrm{kg}$} & \multicolumn{2}{|c|}{$300 \mu \mathrm{g} / \mathrm{kg}$} \\
\hline & $1^{\text {st }}$ & $2^{\text {ad }}$ & $1^{\text {st }}$ & $2^{\text {nd }}$ & $1^{\text {st }}$ & $2^{\text {nd }}$ \\
\hline$r(\log ([T H C]+1)$, SDLP $)$ & -.23 & -.32 & -.26 & .01 & .13 & .07 \\
\hline$r(\log ([T H C]+1), S P)$ & $-.68^{* *}$ & $-.72^{*}$ & $-.45^{\circ}$ & -.39 & -.52 & $-.58^{*}$ \\
\hline$r(\log ([\mathrm{THC}]+1), \mathrm{SDSP})$ & -.37 & $-.57^{* *}$ & $-.52^{*}$ & -.38 & -.27 & -.22 \\
\hline$r\left(\log ([\mathrm{THC}]+1), \lambda_{c}\right)$ &. .33 & $.51^{\circ}$ & .24 & .35 & .08 & .33 \\
\hline$r(\log ([T H C]+1)$, Side Contacts $)$ & .16 & .24 & .04 & -.19 & .31 & $.47^{*}$ \\
\hline$r\left(S D L P, \lambda_{c}\right)$ & $-.47^{\circ}$ & $-.50^{\circ}$ & $-.42^{\circ}$ & $-.56^{\circ *}$ & $-.47^{\circ}$ & -.27 \\
\hline$r$ (SDLP,Side Contacts) & 41 & .38 & .10 & .41 & 27 & .38 \\
\hline
\end{tabular}

Table 4.5 shows that SDLP was not related to preyailing plasma levels of THC. Another driving performance measure, mean speed, was only moderately, yet consistently, related to THC; subjects having high [THC] values drove slower than those having low [THC] values. Driving performance was moderately related to critical tracking; poorer tracking performance on the road (higher SDLPs) concurred with poorer tracking performance in the laboratory (lower $\lambda_{s} s$ ).

Five percent of the driving tests undertaken in this experiment yielded SDLP scores above the normal limit of $35 \mathrm{~cm}$ (Table 4.6). This limit was established by several hundred young and middle-aged volunteers and psychiatric patients who uniformly failed to achieve higher scores in the same test after being treated with placebo in all of the Institute's studies since 1986. It is illuminating to examine the present drivers who drove over the limit with respect to their prior treatments and the plasma concentrations of THC and its metabolite they exhibited at these times.

Table 4:6 Drug levels and SDLPs from those subjects whose SDLPs exceeded $36.0 \mathrm{~cm}$.

\begin{tabular}{lccccc}
\hline Subject & Condition & Trial & $\begin{array}{l}{[\text { THC] }} \\
(\mathrm{ng} / \mathrm{ml})\end{array}$ & $\begin{array}{l}{[\text { THC-COOH] }} \\
(\mathrm{ng} / \mathrm{ml})\end{array}$ & $\begin{array}{l}\text { SDLP } \\
\text { (cm) }\end{array}$ \\
\hline 1523 & $100 \mu \mathrm{g} / \mathrm{kg}$ & 2 & 2.5 & 3.2 & 38.7 \\
$1503^{*}=$ & $200 \mu \mathrm{g} / \mathrm{kg}$ & 2 & 1.7 & 5.1 & 36.3 \\
1523 & $200 \mu \mathrm{g} / \mathrm{kg}$ & 1 & 8.8 & 6.7 & 39.2 \\
1523 & $200 \mu \mathrm{g} / \mathrm{kg}$ & 2 & 4.3 & 4.3 &. \\
1527 & $200 \mu \mathrm{g} / \mathrm{kg}$ & 2 & 10.0 & 16.2 & 37.0 \\
1506 & $300 \mu \mathrm{g} / \mathrm{kg}$ & 2 & 9.8 & 3.8 & 39.4 \\
1523 & $300 \mu \mathrm{g} / \mathrm{kg}$ & 1 & 18.0 & 11.5 & 36.6 \\
1523 & $300 \mu \mathrm{g} / \mathrm{kg}$ & 2 & 5.2 & 9.2 & 36.9 \\
1526 & $300 \mu \mathrm{g} / \mathrm{kg}$ & 2 & 6.7 & 16.2 & 39.3 \\
\hline
\end{tabular}

These data are important in two respects. Aberrant driving performance never occurred after placebo smoking, only once after the lowest THC dose and with equal frequency ( $4 x)$ after both 
of the higher doses. Moreover, aberrant driving generally occurred during the second and not the first ride in direct opposition to the trend in plasma THC concentrations. From this, it's easy to infer the futility of predicting changes in SDLP, and presumably other aspects of driving performance, from a single [THC] estimation.

Correlations were also computed between drug plasma concentrations and changes in driving performance from placebo to marijuana conditions. These correlations were generally smaller than those involving the raw scores. Thus, [THC] does not predict changes in that performance.

Relationship between Driving Performance and Frequency of Current Use. Subjects were classified into two categories according to the frequency of reported cannabis consumption. Twelve subjects were infrequent users; i.e., between once weekly to once monthly; eleven subjects were classified as frequent users; i.e. at least once weekly but less than daily. SDLP values then entered a repeated measures MANOVA with Frequency of use as a between-groups factor, and Dose and Time as within-subjects factors. MANOVA failed to show a main effect of Frequency; interactions of Frequency and the other factors were also not significant.

Relation between Driving Performance and Driving under the Influence Experience. Subjects were classified into two categories according to the frequency of reported driving within one hour of cannabis consumption (see Table 3.1). SDLP values then entered a repeated measures MANOVA with Experience as a between-groups factor, and Dose and Time as withinsubjects factors. Neither Experience nor any interaction was significant.

Relation of Willineness to Drive to Perceived "high" and Driving Performance. The relationship between willingness to drive and changes in SDLP and perceived "high" was determined as follows. Groups were defined by their willingness to drive; i.e., two groups were defined comprising those subjects who would not have driven and those who would, for each combination of condition (4x), sampling time $(2 x)$ and urgency of circumstance $(3 x)$, separately. Student's 2-tailed t-test for independent means was employed to determine whether the groups had significantly different change scores (drug minus placebo) of SDLP and perceived "high". Thus, 18 different t-test were performed for each variable, SDLP and perceived "high". Criterion for statistical significance was set at .01 because of the large number of tests. No significant differences in SDLP change were found between subjects willing and those reluctant to drive. With respect to changes in perceived "high", only two significant effects were found. Subjects willing to drive under the imagined circumstance $B$ ("important but avoidable") at both sampling times in the $200 \mu \mathrm{g} / \mathrm{kg}$ condition felt less "high" than those who would not drive. However, this observation is not of major concern, since it was not supported in the other conditions and circumstances. From these results, it can therefore be concluded that subjects' willingness to drive was not related to either perceived "high" or driving performance.

\section{DISCUSSION}

This study demonstrated that marijuana impairs driving performance as measured by an increase in SDLP; all three THC doses significantly affected SDLP relative to placebo. It is remarkable that driving impairment was about the same after the two higher doses. This cannot be due to a ceiling effect, since greater deterioration in road tracking performance has been found after 
many prescription drugs (e.g. Robbe et al., 1989) and also high doses of alcohol (Louwerens et al., 1987). One possible explanation for the lack of a clear dose related impairment may be that subjects were able to mitigate the effects of the highest dose by "trying harder". Indeed, subjects reported putting more effort in performing the test after smoking the highest THC dose. In other words, subjects tried to overcome the perceived disparity between their actual state and the one required to drive the car efficiently; the higher the THC dose, the greater the disparity between the actual and required states, and the harder they tried to compensate for it.

Though marijuana's adverse effects on SDLP were somewhat smaller in the second test than in the first, no significant interaction was found between the treatments and repetition of the test. This means that the driving performance decrement after smoking marijuana persisted almost undiminished for two hours after smoking.

What is the practical relevance of the size of the effects of marijuana upon lateral position variability? This can be inferred by comparing marijuana's effects to those of alcohol in the same test. As cited above, Louwerens et al. (1985, 1987) conducted a study to establish the dose-effect relationship of alcohol. Their study resembled the present one in many aspects: they applied exactly the same driving test, the study was performed on a closed road, and involved the participation of 12 male and 12 female volunteers. They were able to derive an empirical equation for predicting the change in SDLP from placebo levels with increasing blood alcohol concentrations. Mean SDLP began to change significantly at blood alcohol concentration of $0.03 \mathrm{~g} \%$ and increases exponentially to the point where the vehicle's lateral motion can no longer be restricted to within lane boundaries (at about $\mathrm{BAC}=0.12-0.15 \mathrm{~g} \%$, on the average).

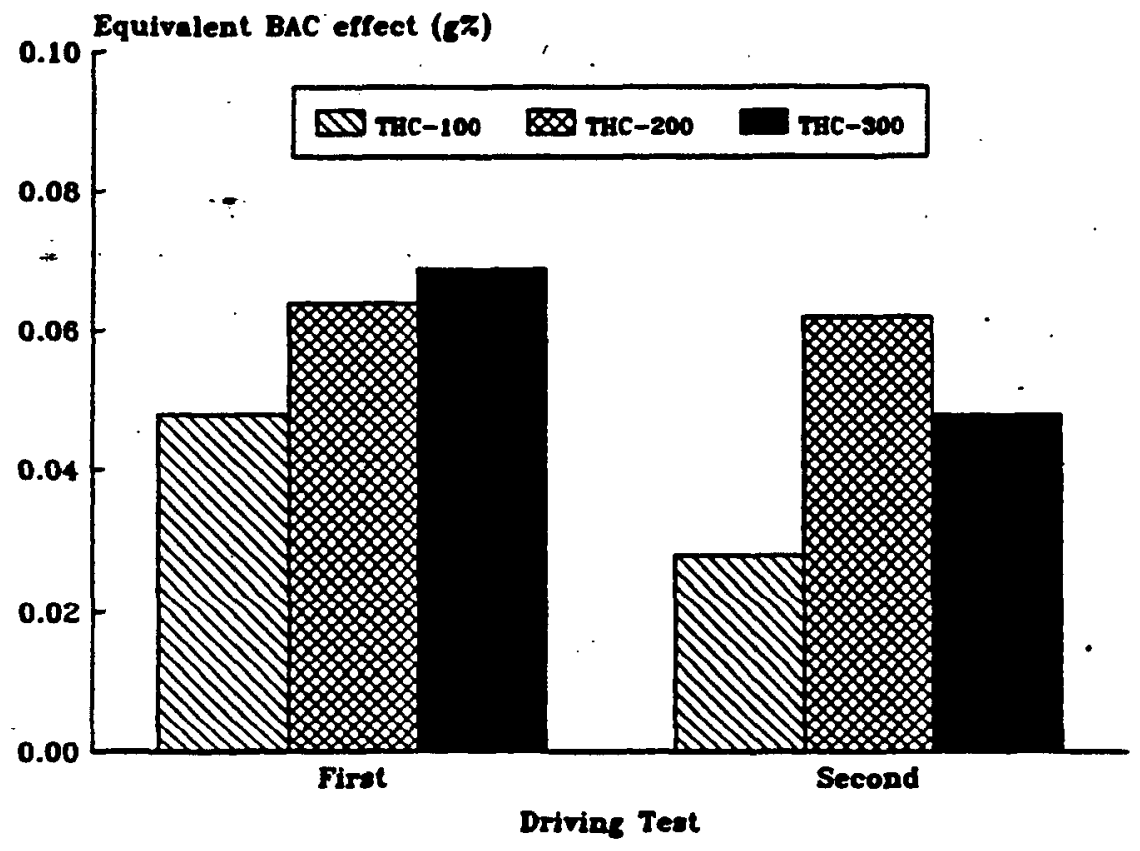

Figure 4.7 Comparison of mean SDLP changes after marijuana smoking to those associated with BAC.

Elevations of the group's mean SDLP in the first and second test after smoking 100, 200 and $300 \mu \mathrm{g} / \mathrm{kg}$ are presented in Figure 4.7 , but now as their respective BAC equivalent 
producing the same driving impairment. Two tentative conclusions can be drawn from these data: first, small doses of THC are capable of impairing driving performance; secondly, performance deterioration is only little worse, yet equivalent to BACs over the Dutch legal limit of $0.05 \mathrm{~g} \%$, when much higher doses are smoked.

As mentioned above, mean SDLP values did not change greatly over time. The same can be said of mean subjective driving quality ratings. Yet other parameters did change over time; THC in plasma, perceived "high", heart rate elevation and perceived effort to accomplish the driving test were all less in the second test than in the first. Another important observation was made when the 5\% highest SDLPs were examined in greater detail. Nearly all of these scores were recorded in the second rather than the first driving test after smoking higher THC doses. It may be that subjects did not try to compensate as much during the second ride because they felt less intoxicated. Whatever the reason, these results clearly indicate that the behaviorally toxic effect of THC had not yet dissipated. This means that "behavioral intoxication" may outlast physiological and subjective marijuana intoxication, a phenomenon already reported by other investigators (e.g. Reeve et al., 1983; Yesavage et al., 1985). On the other hand, subjects' pefceived driving performance, measured retrospectively, did not change from the first to the second test when compared to placebo. Thus, while they felt less intoxicated in the second test they realized that their driving impairment was still the same as in the first test. Subjective feelings of "high" should therefore not be equated with subjective feelings of impairment, as is often done.

Some investigators (Klonoff, 1974; Hansteen et al., 1976) found that marijuana impairs lateral position control as measured by number of cones hit in slalom tests. Others (Casswell, 1979; Attwood et al., 1981) did not find any effect of marijuana upon lateral position control as measured in a similar way as in the present study, presumably due to the low THC dose (6.25 mg in Casswell's study) or the small number of subjects (eight in Attwood's study). Peck $e t$ al. (1986) found that the number of cones hit decreased after smoking marijuana, probably due to a reduction in speed. Most of these studies also measured alcohol's effects (BACs between 0.04 and $0.10 \mathrm{~g} \%$ ) on lateral position control. It was generally concluded that marijuana's effects were less than alcohol's, especially at BACs of $0.08 \mathrm{~g} \%$. Marijuana's effects on lateral position variability were significant, yet not dramatic, in the present study and always less than or equal to the equivalent BAC effect of $0.07 \mathrm{~g} \%$. The reduction in mean speed, though small, fits also well with previous findings. It can therefore be concluded that this study's results are in close accordance with previous closed-course driving studies of THC effects on vehicle handling parameters. This implies that simple psychomotor functions involved in driving are impaired by normally consumed THC doses, though not to such an extent that traffic safety is seriously compromised.

Yet disturbing observations of two individuals' attentional deficits were observed; a sudden loss of the ability to shift attention from the prescribed task to an unexpected event (screwdriver on the road) and the lack of anticipation for a normal event (end of circuit). Since perception and attention are important aspects of actual driving, these instances may indicate an unusually hazardous property of THC when the drug is consumed shortly before operating a vehicle. Therefore, the revised conclusion must be: what was measured was only moderately affected by THC, but another major deficit may have existed after the highest dose which bears further examination. One can not conclude whether the standard driving test applied in this study measures the most important deficits. 
Important practical implications of the study are whether driving performance decrements can be predicted by prevailing plasma concentrations of the drug. Though average [THC] and [THC-COOH] values were clearly dose-related, driving impairment reached a ceiling before the highest concentrations were achieved. Inter-subject correlations between plasma concentrations of the drug and driving performance after every dose were essentially nil, partly due to the peculiar kinetics of THC. It enters the brain relatively rapidly, although with a perceptible delay relative to plasma concentrations. Once there, it remains even at a time when plasma concentrations approach or reach zero. The conclusion is that driving impairment cannot be predicted by prevailing plasma concentrations of THC or THC-COOH.

Another way of predicting driving impairment was explored; namely, by performance in laboratory tests that might be potential "roadside" tests. Hand steadiness was impaired by marijuana after high doses but not after $100 \mu \mathrm{g} / \mathrm{kg}$. Performance in this test was not related to driving performance: Previous studies employing the same test (Clark et al., 1970; Milstein et al., 1975) showed greater sensitivity to marijuana-induced impairment of hand steadiness. In those studies subjects were not allowed to rest their hands on the table, which is probably the major reason for the observed difference. In fact, the present and the preceding study merely measured finger rather than hand steadiness. The conclusion must be that hand steadiness was not properly tested, but the results suggest that it is not possible to predict driving impairment by means of hand steadiness performance.

Critical tracking performance was another candidate for being a good "roadside" test. Yet this test failed to show any effect of marijuana which is in conflict with prior research conducted by Sharma and Moskowitz (1975) and more recently Moskowitz et al. (1981). They demonstrated that a THC dose of $200 \mu \mathrm{g} / \mathrm{kg}$ impairs critical tracking performance for at least up to 4 hours post-smoking. Peck et al. (1986), however, also reported a failure of marijuana alone ( $1.0 \mathrm{~g}$ cigarette containing $1.9 \%$ THC) to affect $\lambda_{c}$ scores. They hypothesized that their conflicting results could be explained by their subjects' greater cannabis experience and tolerance. Although the same argument could be applied in the present study, it is suspected that the failure to detect significant changes in $\lambda_{r}$ after marijuana smoking is due to the particular version employed in this and the pilot study (this group's mean values after placebo were also considerably lower than those commonly found in healthy volunteers). The device used was a commercial PC/AT version of CTT which was originally programmed on a Commodore-64 computer. The latter has been successfully applied in psychopharmacological research by the authors and their colleagues and hitherto appeared as a very sensitive test for drug-related impairment (e.g. Robbe et al., 1989; Ramaekers et al., 1992a). This study was the first in which the commercial PC/AT version was employed, so the conclusion that the software or hardware was not well designed seems inevitable.

All subjects were willing to undertake the driving tests. But test conditions were artificial and the same individuals may or may not have been willing to drive under normal circumstances. Therefore, one questionnaire inquired into the subject's willingness to drive an automobile when experiencing the same drug effect under "normal" conditions. Subjects' willingness to drive was related both to consumed THC dose and urgency. After smoking a low THC dose, nearly all were willing to drive, especially for urgent reasons. After higher doses, fewer were willing to drive under all circumstances. According to what subjects said, they did not become less cautious after inhaling increasingly large doses (unlike what is often reported about alcohol). On the contrary, their caution increased with intoxication. Still, $50 \%$ of the subjects reported that they would have driven for an unimportant reason shortly after smoking the two highest THC 
doses. Since willingness to drive was not related to objective driving impairment, it may be concluded that at least some of the subjects either were not able to appraise their driving performance before they had actually driven or did not consider their impairment as critical.

It is interesting to compare plasma concentrations of THC found after smoking the highest THC dose in this study with those found in the pilot study. The highest dose administered in the present study was comparable to the average dose the same subjects consumed in the pilot study. Mean plasma concentrations of THC after the highest dose in the present study, determined 30 and 90 minutes after initiation of smoking, were 50 and $40 \%$ higher than those measured in the pilot study after 40 and 100 minutes. This large difference can only be partially explained by the 10 minutes delay between the two stuties' blood sampling schedule. Apparently, subjects smoked more efficiently in this than the previous study. Since most volunteers were used to smoking hashish instead of marijuana, the increased efficiency compared to that in the pilot study may be explained by familiarization with the particular formulation of the drug.

One of the objectives of this study was to determine whether or not the same experimental conditions could be implemented in a study on marijuana's effects on driving on a primary highway public for other traffic. All subjects were willing and able to finish the driving tests without great difficulty. In cases of the exceptional events, the driving instructor was able to control the situation, safely. The effects of marijuana on SDLP were never so large as after many other drugs that had been safely studied in the presence of other traffic. Furthermore, it can be inferred from what subjects said and did that they would not be expected to seek dangerous situations and would be responsive to the instructor's advice. Normal safeguards were deemed sufficient to ensure safety. Hence, the final conclusion was that it would be safe to repeat this study on a normal highway in the presence of other traffic. 


\section{CHAPTER 5 - MARIJUANA AND DRIVING ON A NORMAL HIGHWAY IN TRAFFIC}

\section{INTRODUCTION}

The preceding study showed that the average driving impairment on a closed highway segment was never particularly great after doses of 100,200 and $300 \mu \mathrm{g} / \mathrm{kg}$ THC (equivalent to impairment at 0.05 to $0.07 \mathrm{~g} \% \mathrm{BAC}$ ) making it ethically acceptable to test the same doses' effects on performance in a more natural environment. It seemed essential to do so because the extent to which one can generalize from closed-course testing to the real world is presently unknown. Therefore, the same approach was applied for testing THC's effects on driving performance in a more realistic situation. In particular, the same THC doses were administered to a new group of subjects undertaking similar driving tests, though now on a highway in the presence of other traffic.

The present study was only the second wherein subjects drove in real traffic after receiving THC treatments. It was the first in which subjects inhaled higher "street doses" of THC before driving. Though the preceding study on a closed highway failed to show dramatic effects of high THC doses on high-speed driving, a conservative approach was chosen in designing the present study in order to satisfy the strictest safety requirements. This approach is unique in traffic science but closely follows the procedure used to determine the tolerability of medicinal drugs in human pharmacological research. It is to test THC's effects on actual driving performance in an ascending dose series (below). If any subject would have reacted in an unacceptable manner to a lower dose, he/she would not have been permitted to receive a higher dose.

The major objective of this study was to confirm the relationship between inhaled THC dose and lateral position variability in the context of a standard road tracking test. A secondary objective was to measure performance in another actual driving test (i.e. car following) to determine whether degrees of impairment would correlate between the two tests in a manner indicating a general influence of THC on driving behavior. The third objective was to continue efforts to correlate plasma concentrations of THC and $\mathrm{THC}-\mathrm{COOH}$ with driving performance impairment as measured in both tests.

\section{Subjects}

\section{METHODS}

Sixteen new subjects, equally comprised of men and women, were selected according to the same inclusion/exclusion criteria as before. They were individually trained to perform the driving tests in a preliminary "dress rehearsal". Training at laboratory tests continued until each subject achieved satisfactory, asymptotical performance levels.

Plasma analyses after conclusion of the study showed that one female's plasma contained neither THC nor THC-COOH in any sample. It was concluded that this subject had not inhaled smoke, so her data were excluded from further analyses. Characteristics of the remaining 15 subjects are given in Table 5.1. T-tests for independent samples showed that males were heavier smokes than females $(p<.007)$. There were no other significant differences between the sexes. 
Table 5.1 Mean \pm SD (range) of subjects' characteristics.

\begin{tabular}{lrr}
\hline & $\begin{array}{r}\text { Males } \\
(\mathrm{N}=8)\end{array}$ & $\begin{array}{r}\text { Females } \\
(\mathrm{N}=7)\end{array}$ \\
\hline Age (yrs) & $28.3 \pm 7.4(22-42)$ & $25.0 \pm 4.6(21-34)$ \\
Weight (kg) & $70.8 \pm 7.0(61.0-83.5)$ & $66.7 \pm 7.9(55.5-79.0)$ \\
Weight (lb) & $156 \pm 15(134-184)$ & $147 \pm 17(122-174)$ \\
Smoking Experience (yrs) & $8.3 \pm 6.5(2-21)$ & $6.3 \pm 5.9(1-16)$ \\
\# Joints/Month & $8.3 \pm 5.0(1-16)$ & $2.0 \pm 1.4(1-4)$ \\
Driving Experience (yrs) & $6.8 \pm 5.7(2-20)$ & $4.9 \pm 4.6(1-15)$ \\
Driving Experience (km x 1000) & $78 \pm 101(10-320)$ & $38 \pm 66(5-188)$ \\
Driving Experience (mi x 1000) & $48 \pm 63(6-199)$ & $24 \pm 41(3-117)$ \\
\# of Subjects Having Driven within & & \\
1 Hour following Cannabis Consumption: & 4 & 7 \\
less than 5 times & 4 & 0 \\
5 times or more & & \\
\hline
\end{tabular}

\section{Design, Doses and Administration}

The study was conducted according to an ascending dose series design where both active drug and placebo conditions were administered, double-blind, at each of three THC dose levels. THC doses were the same as those used in the previous study, namely 100,200 and $300 \mu \mathrm{g} / \mathrm{kg}$.

The lowest dose and placebo were administered in separate First Level treatment conditions spaced a week apart. Half the group received these treatments in the same order, the others in reverse order. Subjects proceeded to the next dose only if, in the driving instructor's opinion, no severe and potentially unsafe drug effects had occurred; and, if the subject was willing to go on to the next level. The intermediate dose and placebo were administered the same way in the Second Level conditions and the decision to proceed was made on the same grounds. The highest dose and placebo were administered the same way in the Third Level conditions to conclude the study.

Marijuana cigarettes were prepared from batches supplied by NIDA, containing $1.77 \%$ THC for the lowest, $2.64 \%$. THC for the intermediate, and 3.58\% THC for the highest dose. Doses were administered by smoking and cigarettes appeared identical at each level of treatment conditions. Cigarettes were smoked through a plastic holder in a fashion determined by the subject but with the constraint that smoking had to be finished within ten minutes. After cessation of smoking, cigarettes were retained for subsequent gravimetric estimation of THC consumed. These analyses revealed that the average ( $\pm S D$ ) amount of consumed THC in the three marijuana conditions was $6.9( \pm 0.7), 13.8( \pm 1.4)$ and $20.7( \pm 2.2) \mathrm{mg}$, or $100( \pm 4), 204$ $( \pm 7)$ and $299( \pm 7) \mu \mathrm{g} / \mathrm{kg}$, respectively.

\section{Testing Procedures}

Upon arrival at the laboratory, subjects' breath and urine were tested for the presence of alcohol and cannabinoids. If cannabinoids were found in the urine, a blood sample was taken for later verification of the presence of THC. Two subjects at a time commenced smoking at $t=0$ (Table 
5.2). Thirty minutes after onset of smoking the subjects performed a battery of laboratory tests (tracking, hand steadiness and body sway) and yielded a blood sample. They were then transported to a primary highway (A76, different than in the previous study) between the Dutch cities of Maastricht and Heerlen where the driving tests were performed. Two instrumented vehicles, the same as those in the previous study, were employed in this study. One subject started the car following test (below) in the eastward direction whilst the other subject was sitting in the passenger's seat of the preceding car involved in the same test. The test was conducted on a $16 \mathrm{~km}(9.9 \mathrm{mi})$ circuit of the highway and lasted about twelve minutes. At the end of the circuit the car turned at a signalized intersection and parked at a service station, whereupon the subjects reversed roles to repeat the test running in the opposite direction. The new driver reentered the highway and began his/her car following test. After conclusion of the car following test, the subject left the highway at an exit ramp and reentered in the opposite direction on the associated entrance ramp. Thereupon both vehicles parked on the paved shoulder.

Table 5.2 Schedule of activities on test-days.

\begin{aligned} & \hline Relative Time (min) Activity \\ & \hline $0-10$ Smoking \\ & $30-40$ Tracking, Hand Steadiness and Body Sway Tests \\ & $40-45$ Blood Sampling \\ & $55-70$ Car Following Test (1st Subject) \\ & $70-85$ Car Following Test (2nd Subject) \\ & $85-135$ Standard Driving Test (Both Subjects) \\ & $140-155$ Car Following Test (1st Subject) \\ & $155-170$ Car Following Test (2nd Subject) \\ & $190-195$ Blood Sampling \\ & $195-205$ Tracking, Hand Steadiness and Body Sway Tests \\ & \hline\end{aligned}

Both subjects then commenced the standard driving test (below) in separate instrumented vehicles at $t=85$ and $t=88$, respectively. The test circuit was the same as for the car following test. Subjects drove twice around the circuit (in total, $64 \mathrm{~km}$ or $\mathbf{4 0} \mathrm{mi}$ ) without stopping in about 50 minutes. At the conclusion of this test, both subjects participated again in the car following test in the same order as before. Subjects were then transported back to the laboratory where they yielded a blood sample and repeated the test battery.

Two pairs of subjects were tested per test night. One pair performed the driving test in daylight, i.e. between 19.30 and 21.30 hours; the other pair commenced driving at 21.30 hours and finished at 23.30 hours in darkness.

\section{Driving Tests}

The standard test was the same as described in the previous study (Chapter 4) except for its duration and the presence of other traffic. Subjects were instructed to maintain a constant speed of $95 \mathrm{~km} / \mathrm{h}(59 \mathrm{mph})$ and a steady lateral position between lane boundaries in the right traffic 
lane. They were allowed to deviate from this only if it would become necessary to pass a slower vehicle in the same lane. Data from the standard test were analyzed to yield the same performance measures as in the previous study; namely, standard deviation of lateral position (SDLP), mean and standard deviation of speed (SP and SDSP), and standard deviation of steering wheel angle (SDST).

The car following test measures drivers' ability to perceive changes in a preceding vehicle's speed and to react in a manner maintaining a constant headway. It began as the preceding and the following vehicle, respectively driven by one of the driving instructors and the subject, operated in tandem on the slower traffic lane while travelling at a speed of $100 \mathrm{~km} / \mathrm{h}(62 \mathrm{mph})$. The subject was instructed to maintain a $50 \mathrm{~m}$ (164 ft) headway however the preceding vehicle's speed might vary. After driving in this manner for about one minute, the operator of the preceding vehicle released the accelerator pedal allowing its speed to fall to $80 \mathrm{~km} / \mathrm{h}(50 \mathrm{mph})$. Immediately thereafter, the operator of the preceding vehicle accelerated to $100 \mathrm{~km} / \mathrm{h}(62 \mathrm{mph})$. The duration of one deceleration and acceleration maneuver was approximately 50 seconds and six to eight, depending upon traffic density, were executed during one test.

The velocity of the leading vehicle was transmitted via telemetry to a receiver in the following vehicle. This signal, along with the following vehicle's own velocity were recorded in parallel, time-coded files on computer files. These data entered a power spectral analysis for. yielding phase-delay, modulus or gain and coherence between the vehicle's velocities at the maneuver cycle frequency (i.e. $1 / 50 \mathrm{~s}=0.02 \mathrm{~Hz}$ ). The average phase-delay betweèn frequencies of 0.01 and $0.03 \mathrm{~Hz}$., encompassing the frequency of the deceleration and acceleration maneuvers, was then calculated and transformed to the time domain to yield a measure of the subject's average reaction time to the movements of the leading vehicle (RT, in seconds): This was taken as the primary dependent variable from the car following test. Gain and coherence were recorded for control. purposes. If the test was performed according to instructions, gain should have a value of about 1.0, and coherence, $>.90$.

During the trials, 3 to 6 direct measurements of separation distance between the following and leading vehicle were made by means of a S-VHS video recording system which was mounted between the following vehicle's front seats facing forward through the windshield. Images of the rear of the preceding vehicle were acquired prior to each deceleration/acceleration maneuver. The camera's internal clock signal was recorded with the video imagery and also converted in an electronic pulse code for simultaneously computer recording along with the two vehicles' speed.

Video imagery recorded throughout the trials were analyzed off-line using an interactive software routine implemented on a IBM-AT computer. A single frame would show the appearance of the preceding vehicle at the moment it begins to decelerate. Next the coordinates of two target markers, spaced $119 \mathrm{~cm}$ apart on the rear of the leading vehicle were identified on the display. From this information the distance separating the two vehicles or "headway" was calculated according to the equation,

$$
d(\text { in } m)=k(1 \mathrm{~m} / \tan (\theta / 2))
$$

where $\mathrm{k}$ is a proportionally constant and $\theta$ the horizontal angle subtended by the camera lens. Once the starting distance was determined from a single measurement, headway changes during the maneuver were calculated using differential speed according to the equation,

$$
H(t)=\int V_{p} d t-\int V_{f} d t+H_{o}
$$


where headway varies as a function of time $(t)$ according to the difference between integrals of velocities of preceding and following vehicles $\left(\mathrm{V}_{p}, \mathrm{~V}_{\mathrm{f}}\right)$ plus the headway $\left(\mathrm{H}_{0}\right)$ that existed at the beginning of the maneuver. Headway and coefficient of variation of headway (CV-Headway) during maneuvers were taken as secondary dependent variables. The coefficient of variation of headway, and not standard deviation, is preferable because of the latter's confounding by mean headway.

\section{Questionnaires}

The same subjective questionnaires used in the previous study were administered to the subjects in the present study. Subjective feelings of "high", present cognitive and emotional state, and subjects' willingness to drive were assessed before the onset and after the conclusion of the driving tests ( $t=50$ and 175). Subjects were also asked about their perception of the administered treatment, whether it was THC or placebo. At the end of each driving test, subjects were required to retrospectively rate the effort given in performing the test and perceived driving quality. Questionnaires are enclosed in Appendix A.

\section{Laboratory Tests}

Three tests were administered to the subjects: critical tracking, hand steadiness and body sway. These were also administered in the preceding studies, but the equipment or procedures were changed in this study.

1. Equipment, not procedures, changed in case of the critical tracking test (CTT). The test had shown no sensitivity to treatments administered in the preceding studies, in spite of the fact that other investigators had used the same test for showing significant effects of much lower THC doses. The validity of the particular MS-DOS version used in the preceding studies was doubtful. Therefore an older Commodore-64 version was employed in the present study. This version of the CTT had proven its sensitivity to drug-induced sedation in several previous studies conducted by the authors and their colleagues (e.g. Robbe et al., 1989; Ramaekers et al., 1992a). Test duration was approximately 5 minutes and mean $\lambda_{t}$ was the dependent variable.

2. As discussed in the previous chapter, the hand steadiness test examined finger rather than hand steadiness because subjects were allowed to rest their hands on the table. Subjects were not allowed to do this in the present study. The modification was expected to induce greater instability. Therefore, the diameters of holes were increased to avoid ceiling effects of the number of contacts between their sides and the hand-held stylus. The diminishing diameters of the five circular holes were now set to $6.30,4.70,3.90,3.05$ and $2.70 \mathrm{~mm}(0.25,0.19$, $0.15,0.12$ and 0.11 in) respectively. The dependent variable was again the square root of the total number of contacts of the stylus with any side. The test lasted about 3 minutes.

3. Postural instability, or body sway, was measured using the stabilometry method (Kapteyn et al., 1983). It involved the use of a balance platform that measures the location of the vector of force which extends vertically downward from the body's center of gravity and its movement over time. Analog output of force transducers within the platform were digitized 
and analyzed to yield simultaneous measures of lateral and sagittal motion around the vertical axis. Subjects were instructed to maintain a static posture while standing over the center of the balance platform with their feet together. Two, 30-second recordings followed. The first with the subject's eyes open, the second with eyes closed. While standing with the eyes open, the subject was required to fixate on a target mounted on the wall from a distance of $2.0 \mathrm{~m}(6.6 \mathrm{ft})$. The mean area circumscribed by the vertical vector of force (i.e. curve surface, in $\mathrm{mm}^{2}$ ) was taken as the dependent variable (CS-O and CS-C for eyes open and closed, respectively).

\section{Physiological Assessments}

The electrocardiogram (ECG) was measured from precordial leads $\left(\mathrm{RC}_{5}\right)$, and the interbeat interval (IBI) times were registered continuously during the driving tests. Cardiac interval times were analyzed to yield three different parameters, mean IBI, the coefficient of variation (CV-IBI), and relative amplitude in the power density spectrum between the frequencies of .07 and $.14 \mathrm{~Hz}$ (PWR-HR).

CV-IBI, is defined as standard deviation of IBI divided by the mean. It is the best measure of IBI variability in the time domain. The coefficient of variation, and not the standard deviation, of IBI is preferable because of the latter's confounding by mean IBI.

PWR-HR is a variability measure within a restricted region of the frequency domain and calculated from the time series of instantaneous heart rates, which are computed at each successive heart beat. It is defined as the integrated amplitude in the power density spectrum between 0.07 and $0.14 \mathrm{~Hz}$.

Mean interbeat interval, and more frequently its reciprocal, mean heart rate, is frequently used for measuring THC's chronotropic cardiac effect. As shown in the previous study, the measure is valuable for assessing the course of the drug's activity over time. However the other measures, CV-IBI and PWR-HR, possess greater psychological significance. They are alternatively used for estimating relative changes in an individual's mental workload, or more correctly the amount of mental effort he must exert for handling a particular workload. In general, both measures vary inversely with the imposed mental workload or the increase in mental effort which is required to cope with a constant workload while maintaining the same level of performance efficiency under the influence of drugs or fatigue (Mulder, 1980; Mulder and Mulder, 1981).

\section{Blood Sampling}

Blood samples were obtained from the subjects by venepuncture immediately before they were transported to the test site and immediately after their return, or approximately 35 and 190 minutes after initiation of marijuana smoking. Two aliquots containing $10 \mathrm{ml}$ each were heparinized and centrifuged, and the plasma fractions were placed in frozen storage for later assays to determine [THC] and [THC-COOH]. The analytical procedures were the same as those employed in the preceding studies. 
Data Analysis

The first step executed was determining the reliability and consistency of performance and subjective parameters measured in the successive placebo treatment conditions. Test-retest reliability coefficients were determined and mean differences between data collected in successive conditions were tested for significance using repeated measures MANOVA. Previous studies wherein unmedicated subjects performance was repeatedly measured in the standard test have failed to show any significant changes over time intervals as long as one week; and, testretest reliability coefficients for the SDLP measule have always been higher than $r=.80$. However, intervals separating successive placebo tests were longer in the present study and the reliabilities of measures obtained in the car following test had yet to be determined. For these reasons it appeared necessary to check the consistency of the subjects' performance in the present study.

Subsequently, data from drug and placebo conditions on the first, second and third levels were analyzed separately. Performance variables recorded on ratio or interval scales were subjected to repeated measures MANOVA with Sex as a between-groups and Drug (placebo versus marijuana) as a within-subjects factor. The effect of Time of testing ( 2 levels) was, if pertinent, simultaneously tested as a within-subjects factor in the same analyses.

It appeared that marijuana's effects on reaction time in the car following test were confounded by mean headway. Reaction times were therefore also analyzed by means of covariance analysis, using headway as the covariate. Covariance analysis estimates what the scores on one variable (in this case, reaction time) would have been if the same "average" score on another variable (in this case, mean headway) would have occurred in all conditions. It allows one to estimate the effects of THC on reaction time independently of those on headway.

Willingness to drive data were analyzed in the same manner as the parametric data. That is, data from drug and placebo conditions on the first, second and third levels were analyzed separately. Thus, data from both sampling times in a drug condition and its respective placebo condition were simultaneously tested for assessing differences in the proportion of subjects willing to drive for a particular reason.

Separate dose effects were tested using the "Sequential Bonferroni" procedure for adjusting the $\alpha$-probability criterion $\left(p_{c}\right)$ in accordance with the number of separate comparisons in a given set (Overall and Rhoades, 1987). For the largest of three differences tested at once, $p$ had to be less than $.05 / 3=.017$ to be judged significant. For the second, it had to be $<.05 / 2=.025$; and for the smallest difference, $p<.05$. The adjustment had the effect of holding the probability of making a type I error at $p \leq .05$ over the entire set of comparisons.

Though data from both the marijuana and respective placebo condition entered MANOVA, figures illustrating the results display mean difference scores (drug - placebo) and standard errors of the difference (SED). The former are depicted by the height of the bars, the latter by the vertical lines above or below the bars. Difference scores were used in order to keep the figures as simple as possible. As a consequence, main effects of Time could not be depicted in the figures: a parallel rise or fall in the mean levels of a variable over time would not affect their difference. This is, however, not a major problem, since it is not very interesting to know whether subjects' performance changed in both conditions in the same manner. More interesting is a Time by Drug effect, which means that the difference between effects of marijuana and placebo changed over.time. This effect would be obvious in the figures.

Significant Sex effects were generally absent. Results pertaining to differences between the sexes are therefore mentioned only in the exceptional cases where these were significant. 


\section{RESULTS}

No subject dropped out during the experiment, neither on their own initiative nor on the driving instructor's. Therefore, the results presented below include data from fifteen subjects at each level of treatment. It was impossible to obtain every blood sample from one woman, even after repeated attempts. Her data were therefore excluded from all analyses involving drug and metabolite plasma concentrations. However, assays of what samples were obtained indicated that she did inhale THC. Consequently, her data were not excluded from other analyses.

\section{Test-Retest Correlations and Consistency of Performance}

Correlations between measurements obtained from the successive placebo treatment conditions are shown in Table 5.3. Correlations between repeated SDLP's were somewhat lower than those obtained in earlier studies for "normal" subjects, but were still both highly significant and consistent. Mean and standard deviation of speed were also quite reliable. -Steering wheel variability, however, was not a reliable measure.

Table 5.3 Correlations between parameters measured at the same time in the successive placebo treatment conditions $\left({ }^{*} p<.05{ }^{* *} p<.01\right.$; one-tailed).

\begin{tabular}{|c|c|c|c|c|c|c|}
\hline & \multicolumn{2}{|c|}{$1^{\text {st }}$ and $2^{\text {nd }}$ placebo } & \multicolumn{2}{|c|}{$2^{\text {nd }}$ and $3^{\text {nd }}$ placebo } & \multicolumn{2}{|c|}{$1^{\text {st }}$ and $3^{\text {rd }}$ placebo } \\
\hline - & $1^{\text {st test }}$ & $2^{\text {nd }}$ test & $1^{\text {st }}$ test & $2^{\text {nd }}$ test & $1^{\text {st }}$ test & $2^{\text {nd }}$ test \\
\hline \multicolumn{7}{|c|}{ Standard Driving Test: } \\
\hline SDLP & $.75^{*}$ & & $.76^{* *}$ & & $.72 *$ & \\
\hline SP & $.61^{* *}$ & 2 & $.84^{\circ *}$ & $\therefore$ & $.77^{* *}$ & \\
\hline SDSP & $.66^{* *}$ & & $.69^{\circ *}$ & & $.55^{*}$ & \\
\hline SDST & -.21 & & .17 & & -.62 & \\
\hline Subj. Driv. Quality & .24 & & .24 & & .30 & \\
\hline Perceived Effort & $.72 *$ & & $.50^{*}$ & & $.56^{\circ}$ & \\
\hline \multicolumn{7}{|l|}{ Car Following Test: } \\
\hline Headway & $.73^{* *}$ & .31 & $.60^{\circ-}$ & $.90^{\circ *}$ & $.67^{* *}$ & -.01 \\
\hline CV-Headway & .48 & .19 & .36 & .40 & $.55^{\circ}$ & .07 \\
\hline RT & $.61^{\circ *}$ & $.45^{\circ}$ & $.81^{\circ}$ & $.68^{\circ *}$ & $.64^{-*}$ & .09 \\
\hline Subj. Driv. Quality & $.51^{\circ}$ & .06 & $.64^{* *}$ & .28 & $.61^{\circ "}$ & -.06 \\
\hline Perceived Effort & $.72^{*}$ & $.80^{\circ}$ & $.67^{* *}$ & $.69^{* *}$ & .33 & $.71^{* *}$ \\
\hline \multicolumn{7}{|l|}{ Laboratory Tests: } \\
\hline$\lambda_{c}$ & $.68^{\circ-}$ & $.70^{* \bullet}$ & $.73^{* *}$ & $.87^{\circ *}$ & $.67^{* *}$ & $.78^{* *}$ \\
\hline Side Contacts & $.75^{* *}$ & $.80^{* *}$ & $.84^{\circ *}$ & $.66^{* *}$ & $.63^{* *}$ & $.63^{* *}$ \\
\hline CS-O & .43 & $.49^{\circ}$ & .20 & $.62^{* *}$ & .04 & .31 \\
\hline CS-C & .25 & $.56^{\circ}$. & $.71^{* *}$ & .18 & .37 & .41 \\
\hline \multicolumn{7}{|c|}{ Other Subjective Feelings: } \\
\hline Alertness & $.44^{\circ}$ & $.92^{* *}$ & $.88^{\circ *}$ & $.78^{* *}$ & $.59^{\circ}$ & $.75^{\circ *}$ \\
\hline Contentedness & $.52^{\circ}$ & $.79^{* *}$ & $.76^{*}$ & $.77^{\circ *}$ & $.46^{\circ}$ & $.59^{* *}$ \\
\hline Calmness & $.71^{\circ}$ & .34 & $.87^{*}$ & .41 & $.51^{*}$ & .22 \\
\hline
\end{tabular}


Subjective driving quality ratings were also unreliable, probably due to restriction of range; i.e. most subjects naturally rated their driving performance as about normal (100\%) following placebo treatment. Perceived effort ratings were somewhat more reliable, indicating that driving under the conditions of this study was consistently a more effortful occupation for some subjects than others.

Car following parameters were not as reliable as those measured in the standard driving test. Performance in the second ride in the first placebo condition was not related to that in the second ride of the second and third placebo conditions. This means that the test still needs further standardization to reduce the error variance of the measures tested. Ratings of perceived effort in the car following test were only slightly more reliable than those obtained in the standard test. Remarkably, ratings of subjective driving quality were highly reliable in the first car following test, but not in the second.

Hand steadiness and $\lambda_{r}$, but not body sway measures, were highly reliable. Ratings of alertness, contentedness and calmness were moderately reliable. Surprisingly, alertness and contentedness ratings were generally more reliable when measured after driving, in contrast to calmness ratings which were more reliable before the driving tests commenced. Correlations between perceived ratings of "high" were not computed since they were generally very low or zero following placebo.

Mean differences between data collected in successive placebo treatment conditions were tested for significance using repeated measures MANOVA. Only $\lambda_{c}$ changed nearly significantly over successive placebo treatment conditions $\left(\mathrm{F}_{2.12}=3.81 ; p<.052\right)$; subjects' performance in this test improved during the study $\lambda_{c}=4.6,4.8 \& 5.0$, for the respective placebo conditions, averaged across both sampling times). Obviously performance had not reached an asymptotic level during practice trials which preceded the beginning of experimental sessions.

\section{Plasma Concentrations of the Drug}

Table 5.4 shows mean, median and range of [THC] and [THC-COOH] by Dose and Time. Placebo values were not used in the statistical analyses since these were zero in most cases. Therefore, data from all THC conditions were analyzed in one MANOVA. The tabular data indicate that [THC] was related to inhaled THC dose, and fell to about the same level three hours after smoking.

Table 5.4 Mean, median and range of [THC] and [THC-COOH] in $\mathrm{ng} / \mathrm{ml}(\mathrm{N}=14)$.

\begin{tabular}{llllllll}
\hline & & \multicolumn{2}{c}{$100 \mu \mathrm{g} / \mathrm{kg}$} & \multicolumn{2}{c}{$200 \mu \mathrm{g} / \mathrm{kg}$} & \multicolumn{2}{c}{$.300 \mu \mathrm{g} / \mathrm{kg}$} \\
& & $t=35$ & $t=190$ & $t=35$ & $t=190$ & $t=35$ & $t=190$ \\
\hline & mean & 7.9 & 0.7 & 12.0 & 1.1 & 16.1 & 1.5 \\
{$[\mathrm{THC}]$} & median & 6.5 & 0.9 & 10.0 & 1.0 & 15.8 & 1.5 \\
& range & $0.8-17.2$ & $0.0-1.3$ & $1.5-27.1$ & $0.0-2.7$ & $4.7-30.9$ & $0.4-3.2$ \\
& & & & & & & \\
& mean & 8.2 & 4.1 & 12.2 & 7.61 & 15.3 & 10.0 \\
{$[\mathrm{THC}-\mathrm{COOH}]$} & median & 7.4 & 4.1 & 11.2 & 6.4 & 13.0 & 8.2 \\
& range & $1.4-19.4$ & $0.0-12.0$ & $2.0-37.2$ & $0.0-32.2$ & $4.2-39.6$ & $1.5-36.3$ \\
\hline
\end{tabular}


MANOVA confirmed this impression with significant Dose $\left(\mathrm{F}_{2,11}=20.75 ; p<.001\right)$ and Time $\left(\mathrm{F}_{1,12}=54.81 ; p<.001\right)$ effects, and Dose by Time interaction $\left(\mathrm{F}_{2.11}=17.80 ; p<.001\right)$. Males had somewhat higher [THC] than females and the Sex effect approached significance $\left(F_{1.12}=4.60\right.$; $p<.053)$; Sex by Dose interaction was not significant.

Plasma levels of the metabolite, THC-COOH, were about the same as those of THC 35 minutes after initiation of smoking, but did not decline as rapidly. Table 5.4 shows that [THC-COOH] was also dose-related at both sampling times. Significant Dose $\left(F_{2,11}=14.49\right.$; $p<.001)$ and Time $\left(\mathrm{F}_{1,12}=62.50 ; p<.001\right)$ effects, but no Dose by Time interaction, were shown by MANOVA.

\section{Perceived "high"}

Ratings of "high" after placebo were near zero in most cases. Therefore changes occurring after marijuana smoking, relative to placebo, instead of the raw values, were analyzed by MANOVA. Average subjective feelings of "high" were dose-related and greatest just before the first driving test (Figure 5.1). Relative to maximum personal experience, peak levels of intoxication were

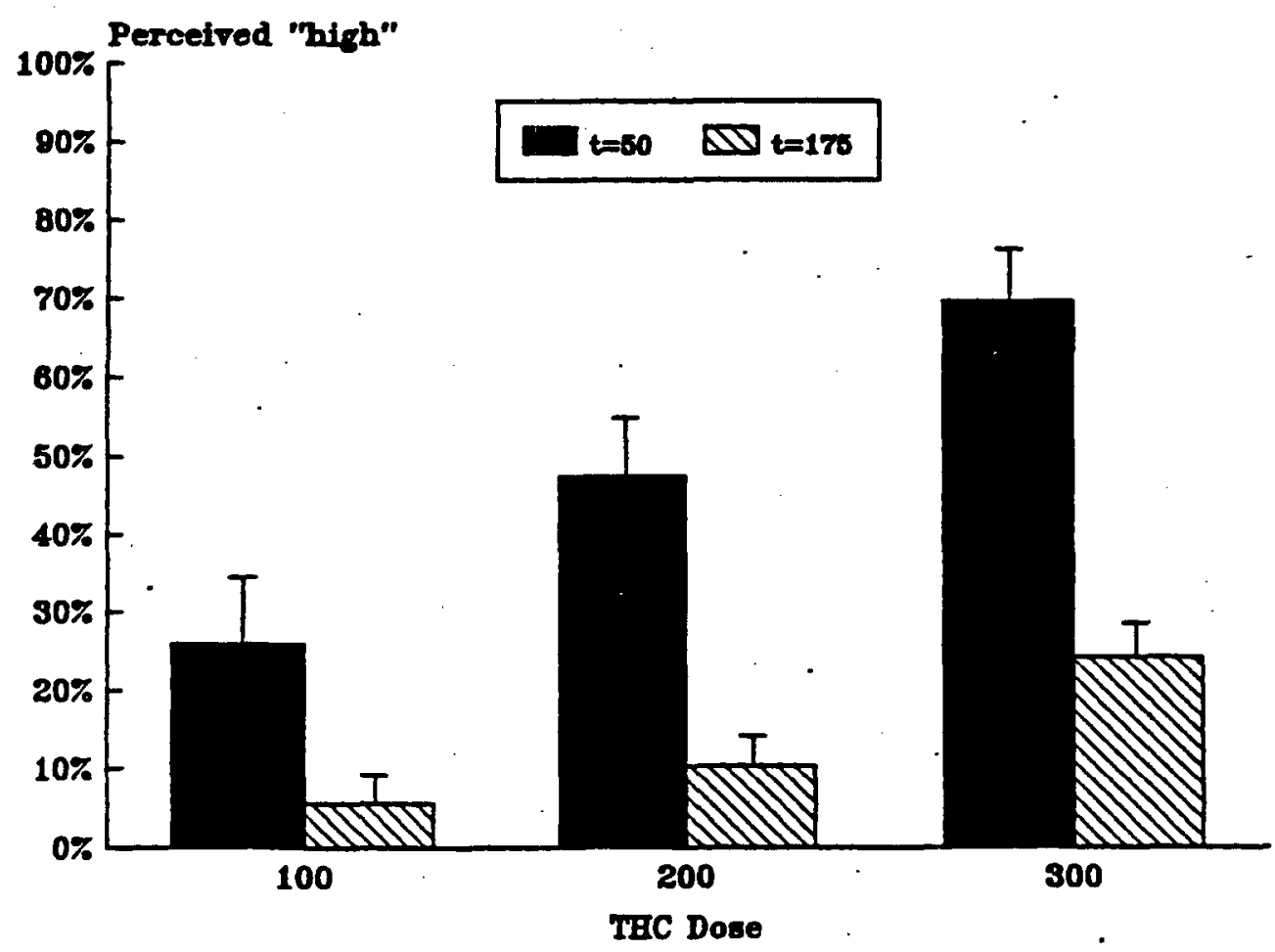

Figure 5.1 Mean (+SED) changes in perceived "high" by Dose and Time, relative to placebo.

about $30 \%, 50 \%$ and $75 \%$ after 100,200 and $300 \mu \mathrm{g} / \mathrm{kg}$ doses. The approximately linear correspondence between the administered dose and average subjective response was impressive.

Change scores were significantly dose-related $\left(\mathrm{F}_{2.12}=35.07 ; p<.001\right)$ and time-related $\left(F_{1.13}=40.25 ; p<.001\right)$. The decline in feelings of "high" over time was also dose-related, resulting in a significant Dose by Time interaction $\left(\mathrm{F}_{2.12}=7.87 ; p<.007\right)$. 
Driving Performance in the Standard Driving Test

All subjects undertook and completed each test in a safe manner; the driving instructors neither terminated any ride prematurely nor intervened while subjects were driving. In other words, the subjects' safety was never compromised.

Figure 5.2 shows the mean changes in SDLP from placebo to marijuana conditions. Performance after consuming THC was worse than after the respective placebo treatment; mean changes in SDLP were $1.1,1.8$ and $2.9 \mathrm{~cm}$ for the 100,200 and $300 \mu \mathrm{g} / \mathrm{kg}$ conditions, respectively. MANOVA showed that SDLP was significantly elevated after inhaling both the higher, but not the lowest, THC doses relative to placebo $\left(F_{1,13}=2.66,6.63 \& 10.16\right.$ for the 100,200 and $300 \mu \mathrm{g} / \mathrm{kg}$ conditions, respectively; $p<.13, .023 \& .007 ; p_{c}=.05, .025 \& .017$ ). No significant differences between males and females were discovered.

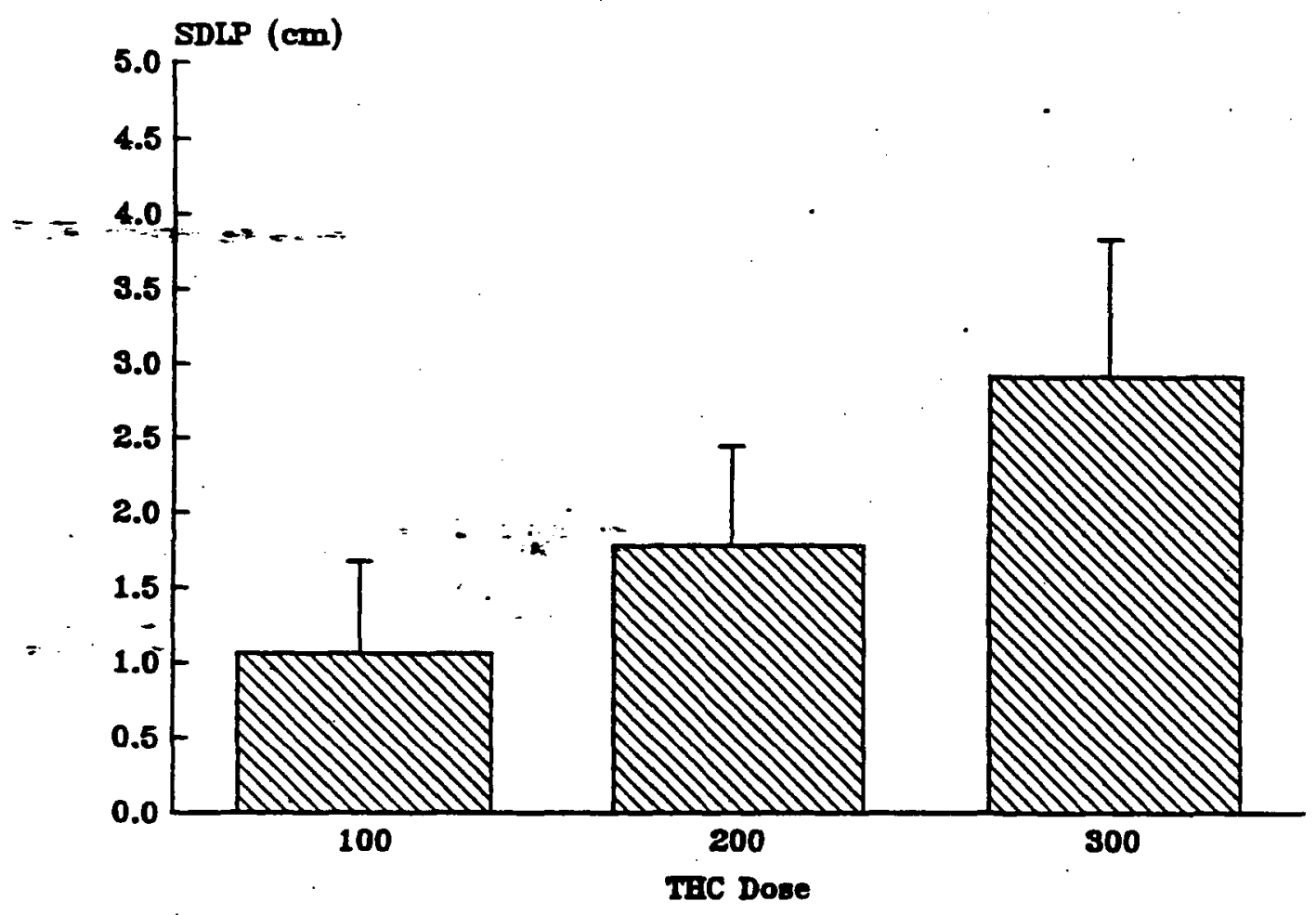

Figure 5.2 Mean (+SED) changes in standard deviation of lateral position in the standard driving test by Drug, relative to placebo.

Mean speed was very close to that established as the target by instructions and varied between conditions from 94.5 to $96.1 \mathrm{~km} / \mathrm{h}(58.7$ to $59.7 \mathrm{mph})$. Subjects drove slower following marijuana than following placebo, but the mean differences were quite small: $0.3,1.1$ and $0.5 \mathrm{~km} / \mathrm{h}(0.2,0.7$ and $0.3 \mathrm{mph})$ for 100,200 and $300 \mu \mathrm{g} / \mathrm{kg}$ conditions, respectively. MANOVA showed that only the change after the intermediate dose was significant $\left(F_{1.13}=8.05\right.$; $p<.014 ; p_{c}=.017$ ).

Standard deviation of speed was very small in each condition, on average $2.5 \%$ of mean speed. Though speed variability increased after smoking THC, the effects were minor and not 
significant. Standard deviations of steering wheel movements were also not affected by THC; mean changes after smoking THC were essentially nil.

Subjects rated their driving performance in the standard test as about "normal" after smoking placebo and after the lowest dose of THC. Ratings were $90 \%$ and $77 \%$ of "normal" driving performance following the 200 and $300 \mu \mathrm{g} / \mathrm{kg}$ conditions, respectively. Changes relative to placebo are shown in Figure 5.3. MANOVA demonstrated that changes in perceived driving quality approached the levels of significance required by the "Bonferroni" adjustment after the two higher THC doses $\left(\mathrm{F}_{1,13}=5.29 \& 5.42\right.$ for the 200 and $300 \mu \mathrm{g} / \mathrm{kg}$ conditions, respectively; • $\left.p<.039 \& .037 ; p_{c}=.025 \& .017\right)$, but were not significantly different from placebo after the lowest THC dose.

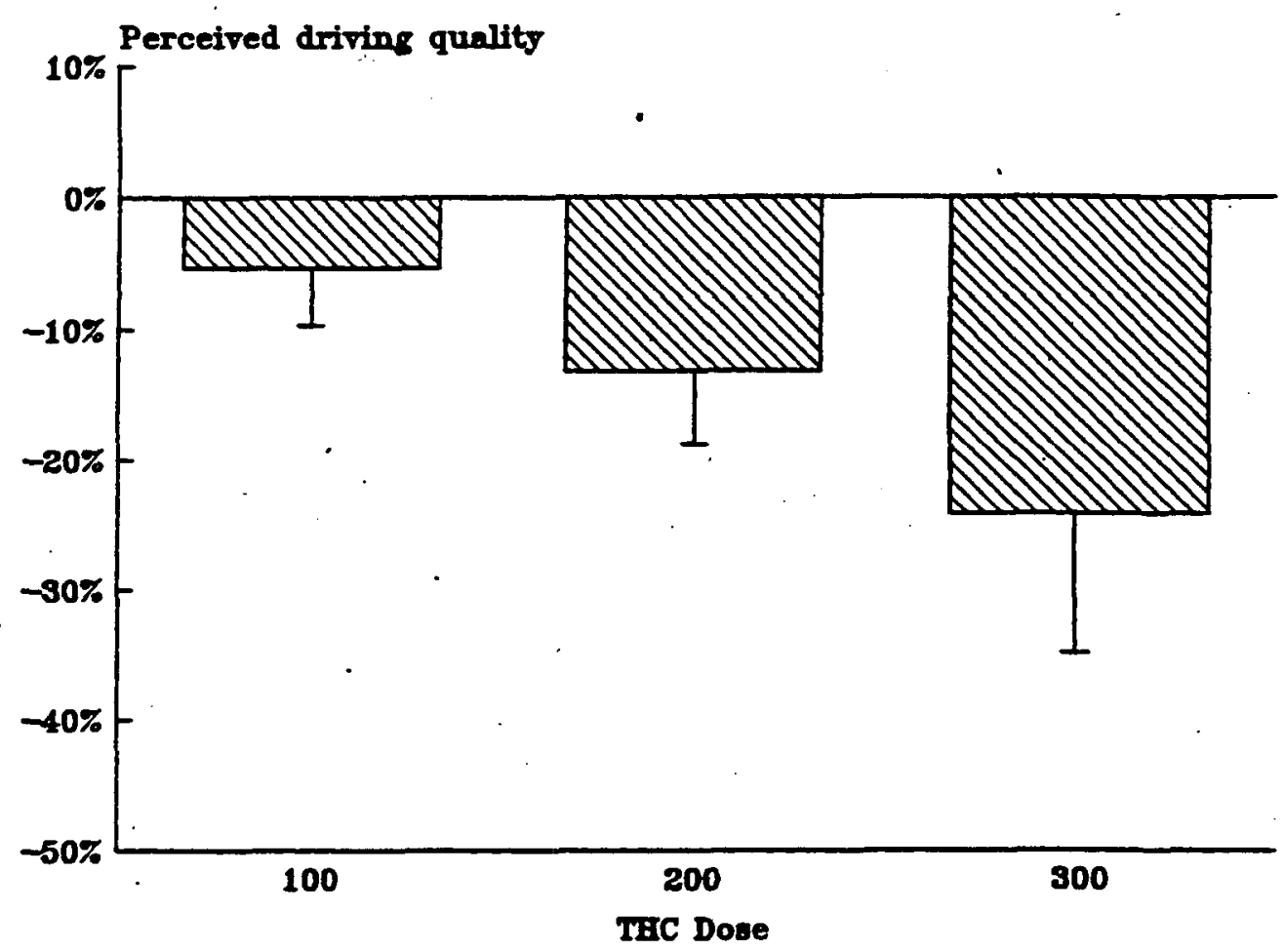

Figure 5.3 Mean (+SED) changes in perceived driving quality in the standard driving test by Drug, relative to placebo. Raw scores were expressed as percentage of "normal".

Changes in perceived effort ratings were trivial after the two lower THC doses. Effort did increase from $29 \%$ after placebo to $39 \%$ following the highest dose. MANOVA failed, however, to reveal any significant effects of THC on perceived effort.

Half of the subjects performed the driving tests in darkness, the other half under daylight conditions. Therefore, repeated measures analysis was again applied to the data, but now with Light/Darkness as a between-groups factor. No significant differences were found between the groups for any variable. 
Driving Performance in the Car Following Test

All subjects were capable of performing the car following test in each treatment condition. There was no need for the driving instructors to ever intervene or prematurely terminate the test. In one instance, a subject needed the driving instructor's help after smoking the highest THC dose. The subject appeared confused when he took his seat in the car prior to the first car following test. The driving instructor noticed the subject's uncertainty as to what he should do and advised him to turn the engine on. Thereafter, the subject had no problems following the instructions and completing the ride.

Mean headway varied between conditions from 44.5 to $54.9 \mathrm{~m}$ (146 to $180 \mathrm{ft}$ ); changes relative to placebo are shown in Figure 5.4. It is interesting to note the inverse relationship between THC dose and headway: the lower the dose the greater the headway, especially in the first test after smoking. MANOVA showed that mean headway was significantly increased after smoking the lowest $\mathrm{THC}$ dose, relative to placebo $\left(\mathrm{F}_{1,13}=7.57 ; p<.016 ; p_{c}=.017\right)$; the two higher doses had no significant effect on headway. No significant Time or Drug by Time effects were found in either THC condition.

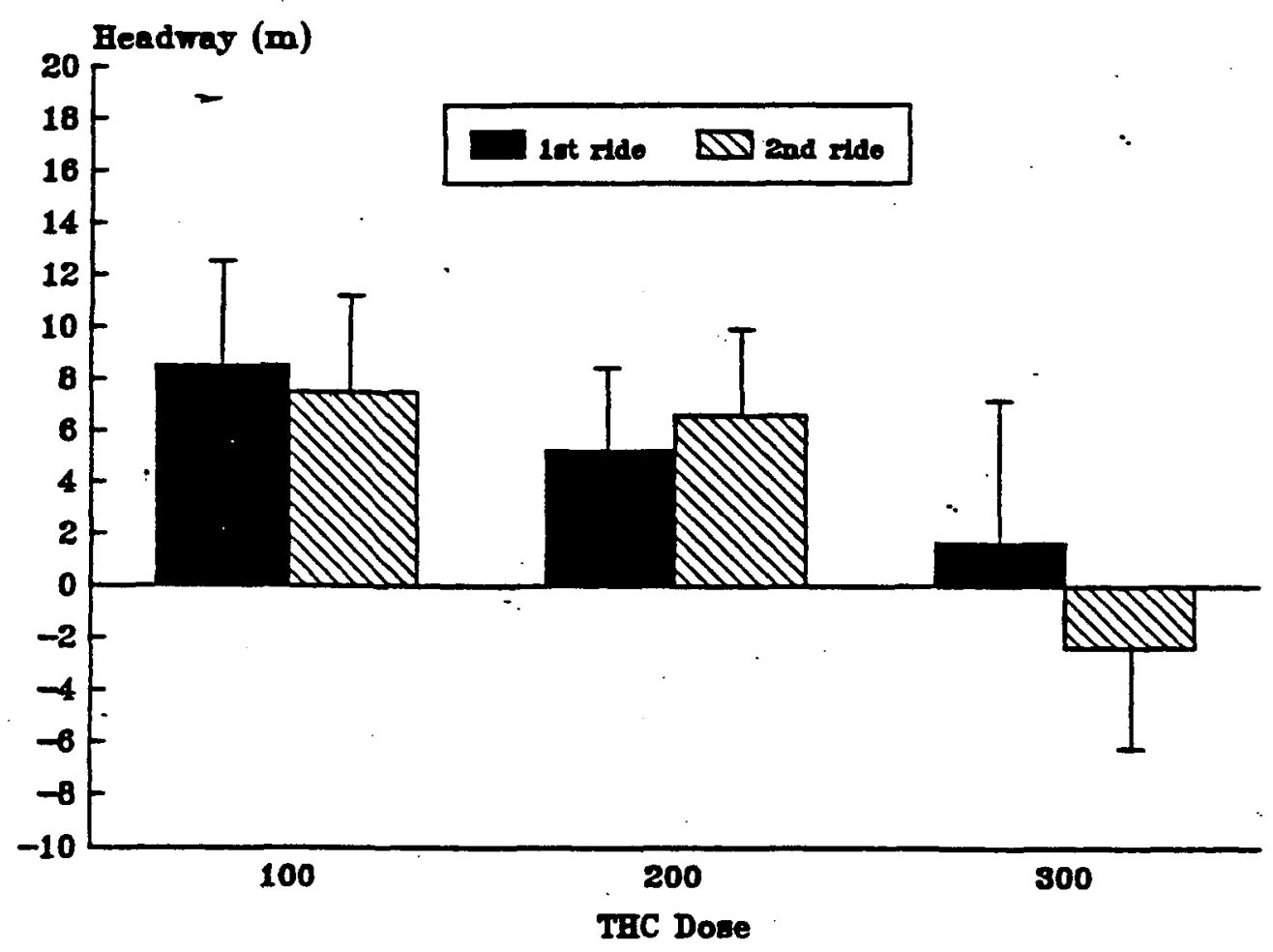

Figure 5.4 Mean (+SED) changes in headway in the car following test by Drug and Time, relative to placebo.

Coefficients of variation of headway, expressed as a percentage, were high and varied between conditions from $18.8 \%$ to $23.7 \%$. Figure 5.5 shows an inverse relationship between CV-Headway and administered dose, with the exception of the first ride after the highest dose. MANOVA revealed a significant effect following the lowest dose $\left(F_{1.13}=16.62 ; p<.001\right.$; 
$\left.p_{c}=.017\right)$, but not the higher doses. Drug by Time interaction approached significance following the highest dose $\left(\mathrm{F}_{1.13}=4.85 ; p<.046 ; p_{c}=.017\right) ; 300 \mu \mathrm{g} / \mathrm{kg}$ THC produced an increased headway variability in the first test after smoking, but not in the second.

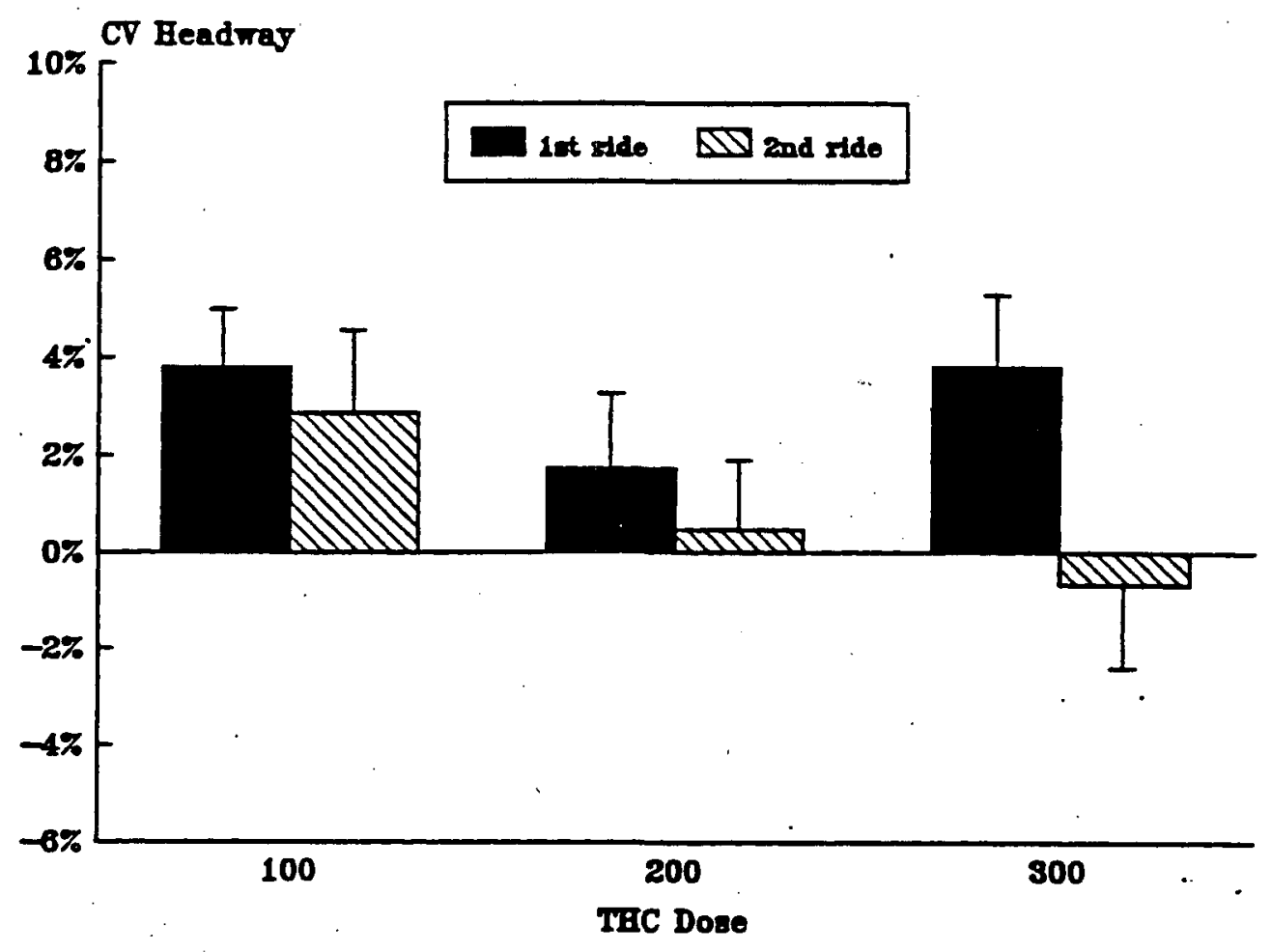

Figure 5.5 Mean (+SED) changes in coefficient of variation of headway in the car following test by Drug and Time, relative to placebo.

Mean. reaction time to perceived changes in the preceding car's speed varied from 1.56 to $2.30 \mathrm{~s}$, between conditions. The changes, shown in Figure.5.6, followed a similar pattern as those of headway; the greatest increase of reaction time occurred after the lowest THC dose, and the smallest after the highest dose. Reaction times were significantly increased after smoking both $100 \mu \mathrm{g} / \mathrm{kg}$ and $200 \mu \mathrm{g} / \mathrm{kg}$ THC, but not $300 \mu \mathrm{g} / \mathrm{kg}\left(\mathrm{F}_{1,13}=10.78,6.26 \& 1.88\right.$, respectively; $\left.p<.006, .027 \& .193 ; p_{c}=.017, .025 \& .05\right)$. Reaction times were, however, strongly related to mean distance ( $r=0.76$, across all conditions). This is understandable: the further apart two cars are, the more difficult it becomes for the subject to perceive changes in the preceding vehicle's velocity.

One may question what the effect of THC on reaction time would have been if subjects had driven at the same mean headway in all conditions? Covariance analysis of reaction time with headway as covariate was applied to answer this question. Figure 5.7 shows the mean changes in adjusted reaction times, from placebo levels. The figure makes clear that the differences in the adjusted reaction times were much less than those in the original ones. Though each THC dose increased reaction time, none did significantly. This means that the elevation in the raw reaction times following the lowest THC dose were simply due to a longer headway. 


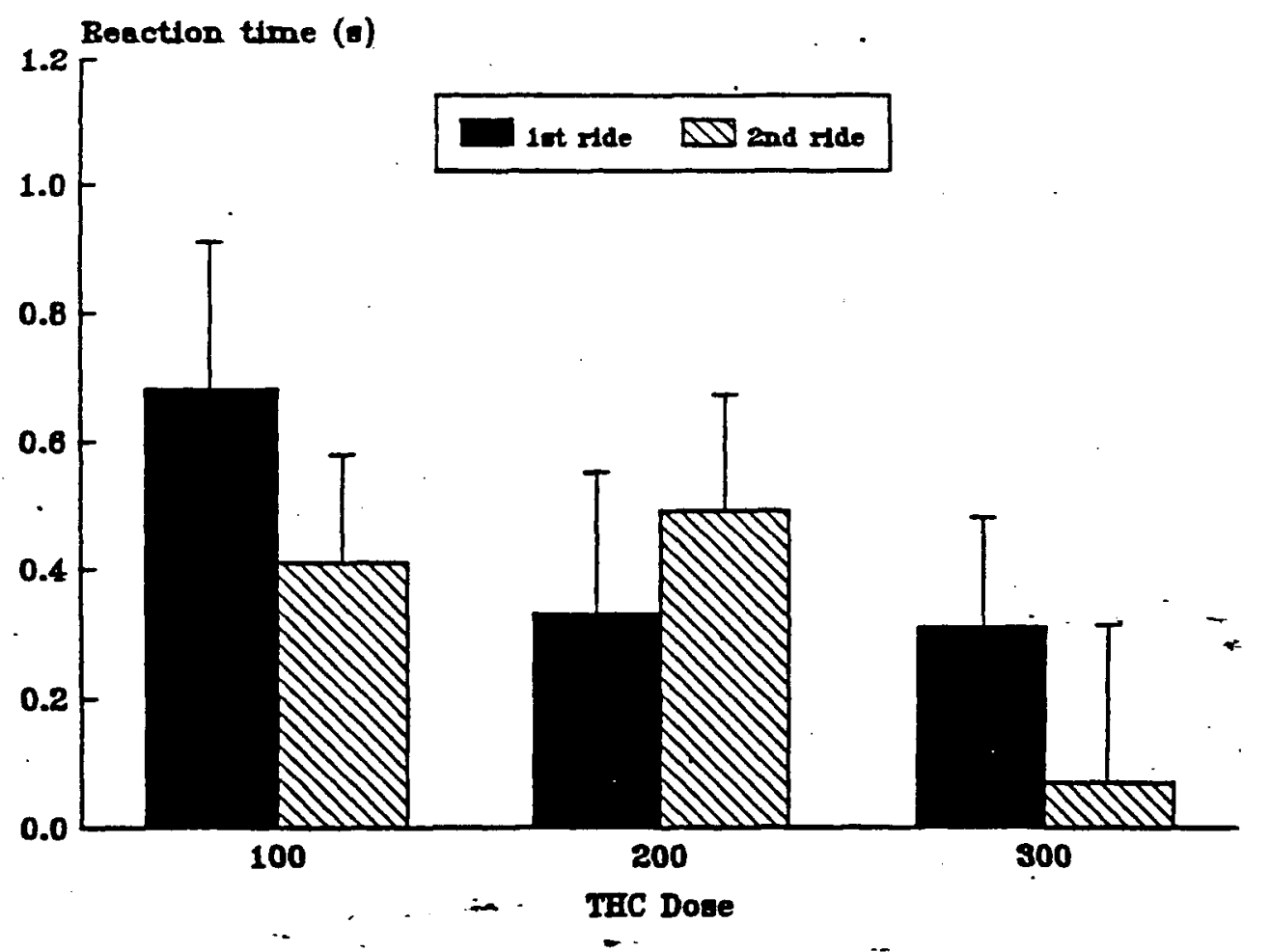

Figure 5.6 Mean (+SED) changes in reaction time in the car following test by Drug and Time, relative to placebo.

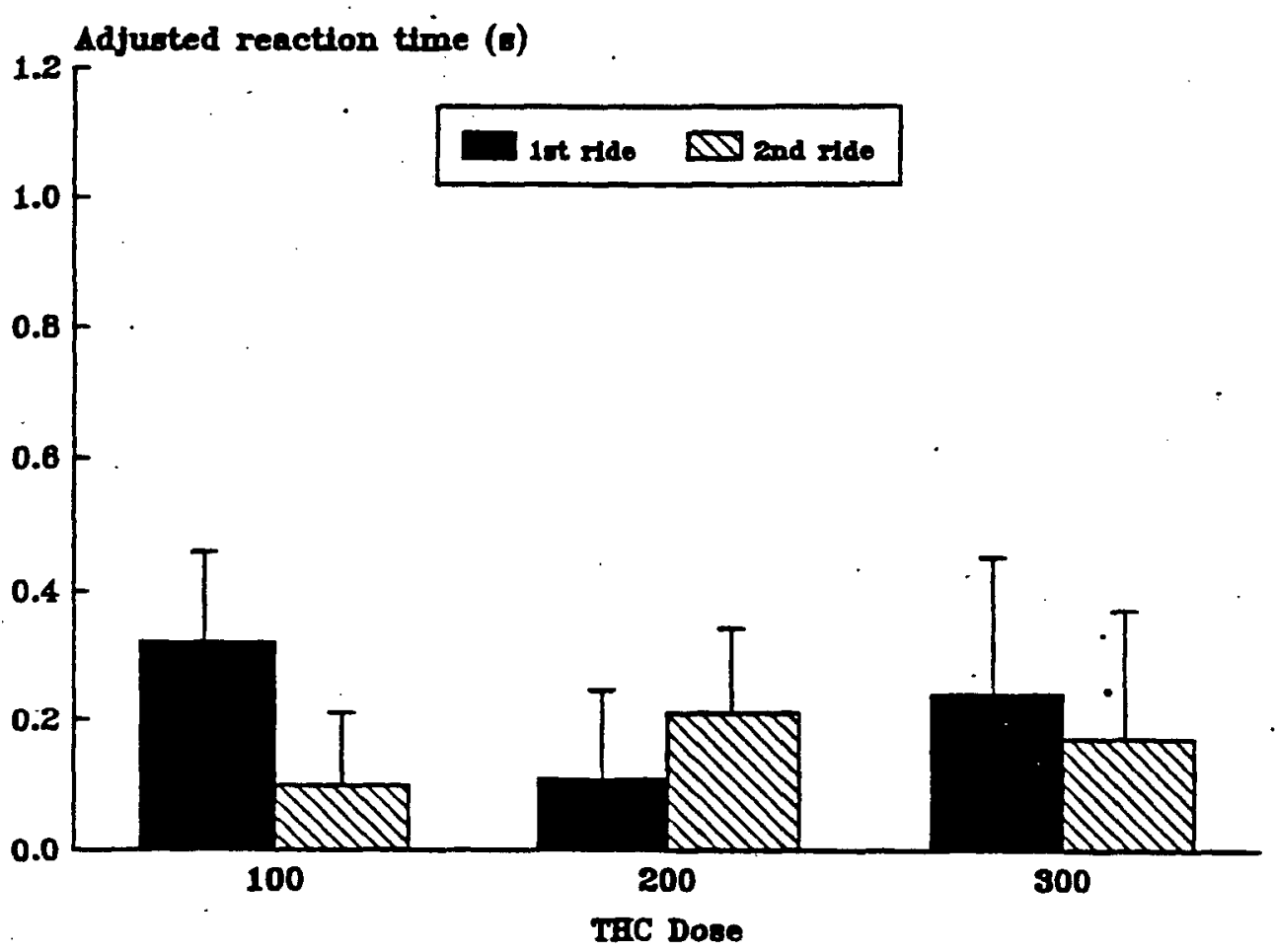

Figure 5.7 Mean (+SED) changes in reaction time, adjusted for changes in headway, in the car following test by Drug and Time, relative to placebo. 
Subjects rated their driving quality in the car following test as somewhat better than normal following each placebo, and worse following each THC dose. Changes from placebo to drug are displayed in Figure 5.8. As for headway and reaction time, there was no clear dose-response

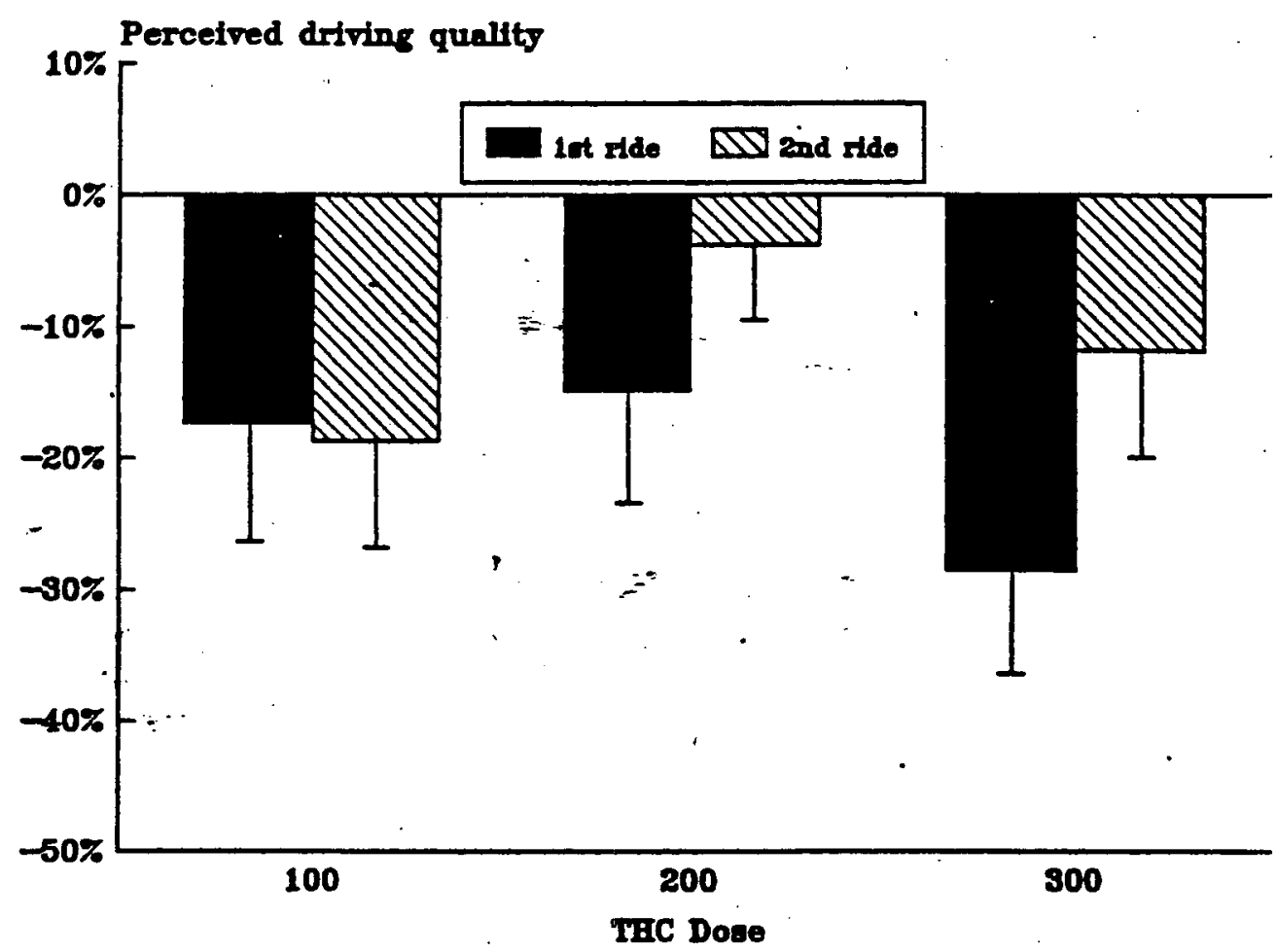

Figure 5.8 Mean (+SED) changes in perceived driving quality in the car $\therefore$ following test by Drug and Time, relative to placebo. Raw scores were expressed as percentage of "normal".

relationship that determined how subjects rated their driving quality. Though they rated it the worst after the highest dose overall their judgement after the lowest dose was nearly as critical. MANOVA demonstrated that driving quality ratings after the highest dose were significantly $\therefore$ different from placebo, but those after the lower doses were not $\left(\mathrm{F}_{1.13}=6.04,3.19 \& 13.84\right.$, for the 100,200 and $300 \mu \mathrm{g} / \mathrm{kg}$ conditions, respectively; $p<.029, .097 \& .003 ; p_{c}=.025, .05 \&$ .017). Neither Time nor Drug by Time effects were significant.

Though perceived effort ratings were generally higher following THC than placebo, MANOVA failed to show any significant drug effect.

\section{Willingness to Drive}

Table 5.5 presents the percentage of subjects willing to drive under specified conditions of different urgencies $(A$. unimportant though gratifying; $B$. important but avoidable; and $C$. urgent). Subjects' responses were similar to those in the previous study. The lower the administered THC dose and the more urgent the reason for driving, the more subjects declared that they would be willing to drive. 
Table 5.5 Percentage of subjects willing to drive under circumstances $A, B \& C$ (see text) by treatment condition and sampling time.

\begin{tabular}{|c|c|c|c|c|c|c|c|}
\hline & & $0 \mu \mathrm{g} / \mathrm{kg}$ & $100 \mu \mathrm{g} / \mathrm{kg}$ & $0 \mu \mathrm{g} / \mathrm{kg}$ & $200 \mu \mathrm{g} / \mathrm{kg}$ & $0 \mu \mathrm{g} / \mathrm{kg}$ & $300 \mu \mathrm{g} / \mathrm{kg}$ \\
\hline A & $\begin{array}{l}t=50 \\
t=175\end{array}$ & $\begin{array}{l}93 \\
93\end{array}$ & $\begin{array}{l}53 \\
80\end{array}$ & $\begin{array}{l}93 \\
80\end{array}$ & $\begin{array}{l}47 \\
87\end{array}$ & $\begin{array}{l}93 \\
93\end{array}$ & $\begin{array}{l}27 \\
53\end{array}$ \\
\hline B & $\begin{array}{l}t=50 \\
t=175\end{array}$ & $\begin{array}{l}100 \\
100\end{array}$ & $\begin{array}{l}73 \\
80\end{array}$ & $\begin{array}{l}100 \\
100\end{array}$ & $\begin{array}{l}60 \\
100\end{array}$ & $\begin{array}{l}100 \\
100\end{array}$ & $\begin{array}{l}40 \\
67\end{array}$ \\
\hline C & $\begin{array}{l}t=50 \\
t=175\end{array}$ & $\begin{array}{l}100 \\
100\end{array}$ & $\begin{array}{l}93 \\
93\end{array}$ & $\begin{array}{l}100 \\
100\end{array}$ & $\begin{array}{l}93 \\
93\end{array}$ & $\begin{array}{l}100 \\
100\end{array}$ & $\begin{array}{l}73 \\
87\end{array}$ \\
\hline
\end{tabular}

Cochran's test showed that the numbers of subjects who would have driven for an unimportant reason following every $T H C$ dose were significantly less than after placebo $\left(Q_{d f=3}=12.0,13.4\right.$ \& 21.6 for the 100,200 and $300-\mu \mathrm{g} / \mathrm{kg}$ conditions, respectively; $p<.008, .004 \& .0001$; $\left.p_{c}=.05, .025 \& .017\right)$. The same held true for the somewhat more important reason $\left(Q_{d f=3}=10.2,18.0 \& 21.4\right.$ for the 100,200 and $300 \mu \mathrm{g} / \mathrm{kg}$ conditions, respectively; $p<: 02$, $\left..0004 \& .0001 ; p_{c}=.05, .025 \& .017\right)$. In contrast, the numbers of subjects who would have driven for an urgent reason after every THC dose were not significantly different from placebo $\left(Q_{d f=3}=3.0,3.0 \& 9.4\right.$ for the 100,200 and $300 \mu \mathrm{g} / \mathrm{kg}$ conditions, respectively; $p<.40, .40 \&$ $\left..024 ; p_{r}=.05, .025 \& .017\right)$.

\section{Perceived Alertness, Contentedness and Calmness}

Table 5.6 shows the mean subjective ratings of alertness, contentedness and calmness, presented as the percentage of maximum scale. Mean subjective ratings generally declined after smoking marijuana, relative to placebo, indicating that subjects then felt less alert, content and calm. MANOVA showed that subjects felt significantly less alert after smoking THC $\left(F_{1,13}=12.57\right.$, $6.48 \& 21.24$ for the 100,200 and $300 \mu \mathrm{g} / \mathrm{kg}$ conditions, respectively; $p<.004, .024 \& .001$; $p_{\mathrm{c}}=.025, .05 \&$.017). Furthermore, a significant Drug by Time interaction was found for the $100 \mu \mathrm{g} / \mathrm{kg}$ condition $\left(F_{1,13}=13.78 ; p<.003 ; p_{c}=.017\right)$, due to the recovery in feelings of alertness from the first to the second time of testing.

Contentedness ratings were only significantly reduced after smoking the highest THC dose $\left(\mathrm{F}_{1,13}=10.20 ; p<.007 ; p_{c}=.017\right)$. Drug by Time interaction was significant in the $100 \mu \mathrm{g} / \mathrm{kg}$ condition $\left(\mathrm{F}_{1,13}=7.71 ; p<.016 ; p_{c}=.017\right)$. Calmness ratings also declined after the higher THC doses $\left(\mathrm{F}_{1.13}=6.05 \& 14.65\right.$ for the 200 and $300 \mu \mathrm{g} / \mathrm{kg}$ conditions, respectively; $p<.029 \& .002$; $p_{c}=.025 \&$.017), and Drug by Time interaction approached significance in all conditions $\left(\mathrm{F}_{1.13}=4.33,6.62 \& 5.10\right.$ for the 100,200 and $300 \mu \mathrm{g} / \mathrm{kg}$ conditions, respectively; $p<.058$, $\left..023 \& .042 ; p_{\varepsilon}=.05, .017 \& .025\right)$.

In summary, subjects felt less alert, content and calm after smoking each THC dose, relative to placebo. The effects were strongest and persisted throughout the testing session after smoking the highest dose. 
Table 5.6 Mean (SE) ratings of alertness, contentedness, and calmness by treatment condition and sampling time.

\begin{tabular}{|c|c|c|c|c|c|c|c|}
\hline & & $0 \mu \mathrm{g} / \mathrm{kg}$ & $100 \mu \mathrm{g} / \mathrm{kg}$ & $0 \mu \mathrm{g} / \mathrm{kg}$ & $200 \mu \mathrm{g} / \mathrm{kg}$ & $0 \mu \mathrm{g} / \mathrm{kg}$ & $300 \mu \mathrm{g} / \mathrm{kg}$ \\
\hline Alert & $\begin{array}{l}t= \\
t=\end{array}$ & $\begin{array}{l}78.3(3.7) \\
69.6(5.0)\end{array}$ & $\begin{array}{l}59.0(4.3) \\
64.7(4.9)\end{array}$ & & $\begin{array}{l}63.9(4.1) \\
66.1(3.6)\end{array}$ & $\begin{array}{l}80.0(3.4) \\
70.6(4.3)\end{array}$ & $\begin{array}{l}57.7(4.6) \\
56.9(6.2)\end{array}$ \\
\hline Content & $\begin{array}{l}t=50 \\
t=175\end{array}$ & $\begin{array}{l}79.3(2.7) \\
72.4(3.4)\end{array}$ & $\begin{array}{l}66.2(5.1) \\
70.3(4.3)\end{array}$ & $\begin{array}{l}77.6(3.3) \\
74.9(4.4)\end{array}$ & $\begin{array}{l}73.6 \\
73.1\end{array}$ & $\begin{array}{l}78.3(3.4) \\
76.7(3.2)\end{array}$ & $\begin{array}{l}67.2(4.4) \\
65.7(4.9)\end{array}$ \\
\hline Calm & $\begin{array}{l}t=50 \\
t=175\end{array}$ & $\begin{array}{l}78.2(4.2) \\
72.5(5.0)\end{array}$ & $\begin{array}{l}64.2(5.2) \\
72.4(3.8)\end{array}$ & $\begin{array}{l}79.5(4.4) \\
75.9(4.1)\end{array}$ & $\begin{array}{l}61.7(6.8) \\
71.3(4.0)\end{array}$ & $\begin{array}{l}79.5(3.6) \\
77.5(3.5)\end{array}$ & $\begin{array}{l}59.6(5.8) \\
70.5(4.8)\end{array}$ \\
\hline
\end{tabular}

\section{Critical Tracking Test}

As shown in Figure 5.9, mean $\lambda_{c}$ measured 30 minutes after initiation of smoking diminished in a dose-related manner. Measured 31/4 hours after initiation of smoking, $\lambda_{c}$ remained low after

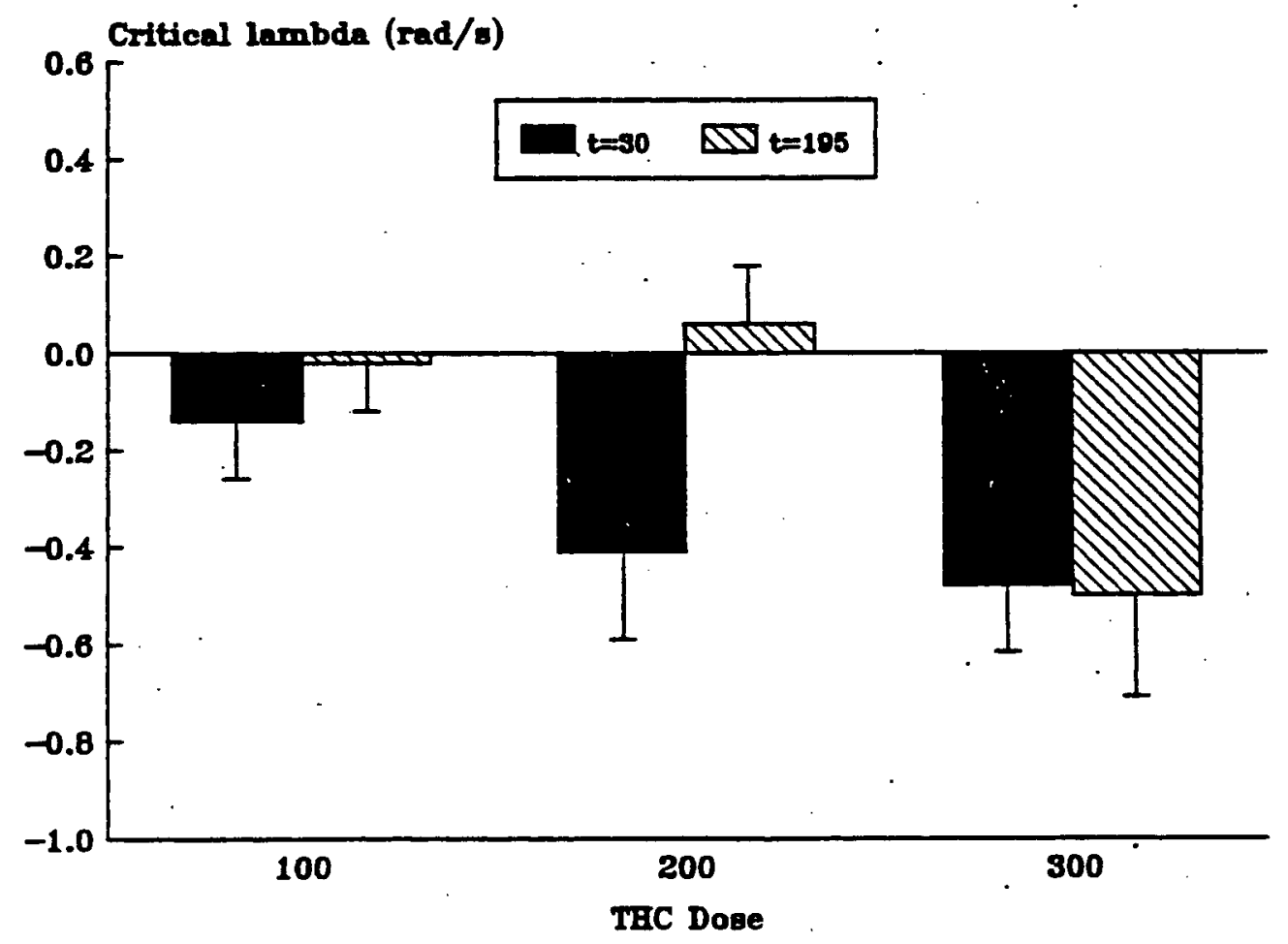

Figure 5.9 Mean (+SED) changes in $\lambda_{c}$ in the critical tracking test by Drug and Time, relative to placebo.

the highest, but not the two lower doses. The lowest THC dose did not affect $\lambda_{c}$ significantly. A nearly significant Drug by Time interaction was found in the $200 \mu \mathrm{g} / \mathrm{kg}$ condition $\left(\mathrm{F}_{1,13}=4.69\right.$; $\left.p<.05 ; p_{c}=.017\right)$; i.e. that dose diminished $\lambda_{c}$ shortly after smoking, but the effect dissipated 
after three hours. The highest dose had a significant effect on $\lambda_{c}\left(\mathrm{~F}_{1.13}=9.03 ; p<.01 ; p_{c}=.017\right)$, both shortly after smoking and three hours later.

\section{Hand Steadiness Test}

Hand steadiness diminished in relation to the dose after marijuana smoking (Figure 5.10). All doses produced significantly greater instability $\left(F_{1,13}=6.72,13.05 \& 45.33\right.$ for the 100,200 and $300 \mu \mathrm{g} / \mathrm{kg}$ conditions, respectively; $\left.p<.022, .003 \& .001 ; p_{c}=.05, .025 \& .017\right)$.

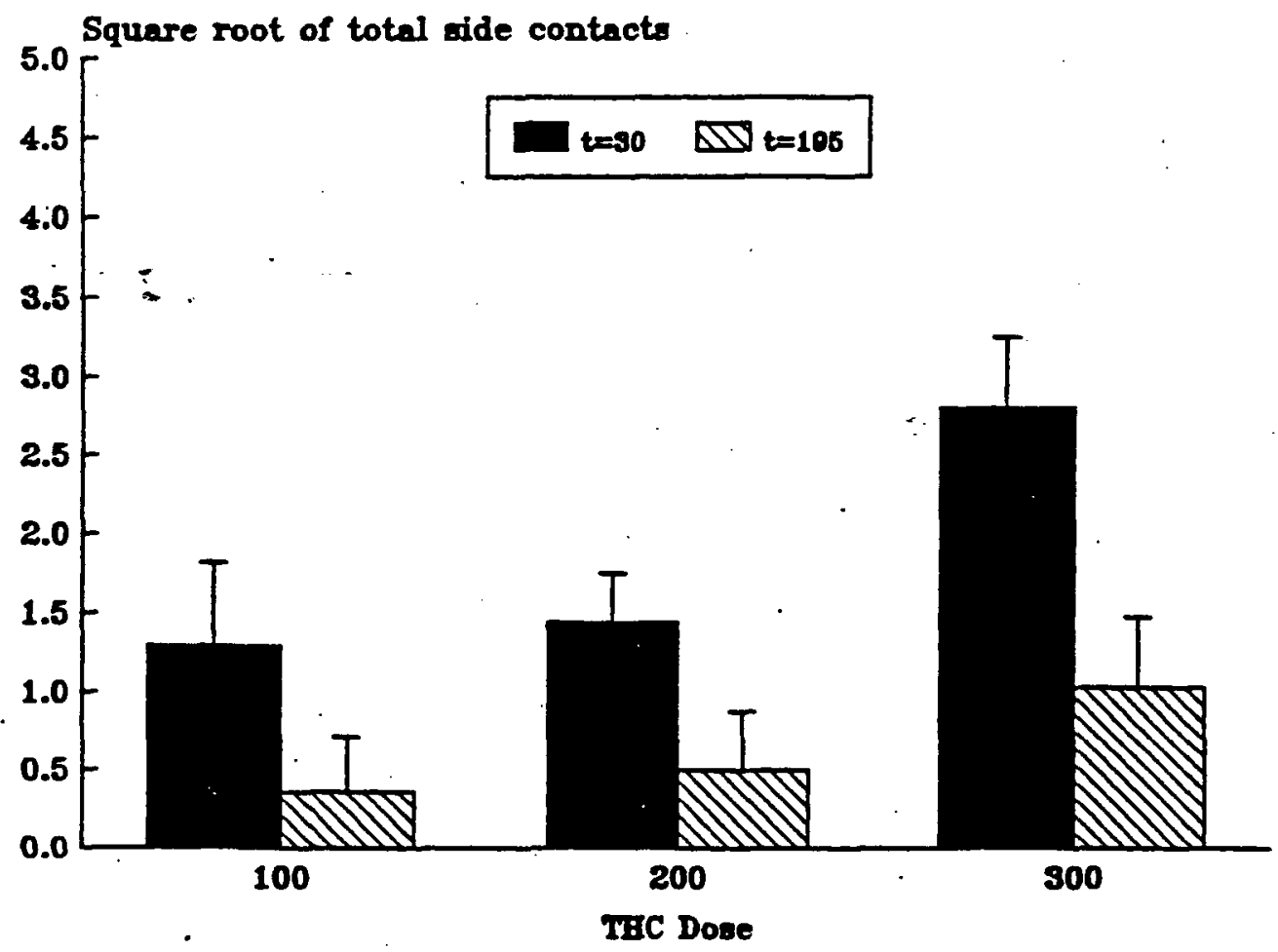

Figure 5.10 Mean (+SED) changes in the square root of total number of side contacts in the hand steadiness test by Drug and Time, relative to placebo.

Instability diminished after three hours, irrespective of the dose, resulting in significant Time effects in each drug-placebo comparison. Drug by Time interaction was not significant after the lowest dose, but approached significance after the higher doses $\left(\mathrm{F}_{1.13}=4.51 \& 6.28\right.$ for the 200 and $300 \mu \mathrm{g} / \mathrm{kg}$ conditions, respectively; $p<.053 \& .026 ; p_{c}=.025 \& .017$ ).

\section{Body Sway}

Mean values for the area circumscribed by the vertical vector of force (i.e. curve surface) are displayed in Table 5.7. Curve surface for subjects standing with eyes open (CS-O) increased after $\mathrm{THC}$, relative to placebo. These effects approached significance $\left(\mathrm{F}_{1,13}=4.67,5.20 \& 4.95\right.$ for the 100,200 and $300 \mu \mathrm{g} / \mathrm{kg}$ conditions, respectively; $p<.050, .040 \& .044 ; p_{c}=.050, .017$ $\&$.025). The greatest change from placebo to marijuana smoking was observed in the $200 \mu \mathrm{g} / \mathrm{kg}$ 
condition, but only in the first test after smoking, resulting in a significant Drug by Time interaction $\left(\mathrm{F}_{1.13}=9.23 ; p<.015 ; p_{c}=.017\right)$.

Table 5.7 Mean (SE) curve surface $\left(\mathrm{mm}^{2}\right)$ in the body sway test, both with eyes open (CS-O) and closed (CS-C), by treatment condition and sampling time.

\begin{tabular}{|c|c|c|c|c|c|c|c|}
\hline & & $0 \mu \mathrm{g} / \mathrm{kg}$ & $100 \mu \mathrm{g} / \mathrm{kg}$ & $0 \mu \mathrm{g} / \mathrm{kg}$ & $200 \mu \mathrm{g} / \mathrm{kg}$ & $0 \mu \mathrm{g} / \mathrm{kg}$ & $300 \mu \mathrm{g} / \mathrm{kg}$ \\
\hline \multirow[t]{2}{*}{ CS-O } & $t=50$ & $29.2(4.5)$ & 38.7 & 26.7 (3.2) & 42.3 & .0) & 4.3) \\
\hline & $t=175$ & $32.1(3.8)$ & $43.3(5.4)$ & $35.5(5.7)$ & $37.3(4.8)$ & $31.7(4.1)$ & $38.5(3.5)$ \\
\hline \multirow[t]{2}{*}{ CS-C } & $t=50$ & $27.9(3.9)$ & $53.0(8.6)$ & $33.7(6.8)$ & $55.2(8.8)$ & $36.1(6.2)$ & 57.7 (11.2) \\
\hline & & $0.3)$ & .3) & (1.1) & & $32.0(0.3)$ & $30.2(3.0$ \\
\hline
\end{tabular}

Mean curve surface for the subjects standing with eyes closed (CS-C), increased markedly in the first but not in the second test. The main effect of Drug approached significance $(p<.10)$ in all three conditions, but Drug by Time interactions were significant for the two lower THC doses $\left(F_{1,13}=8.86,7.82 \& 3.55\right.$ for the 100,200 and $300 \mu \mathrm{g} / \mathrm{kg}$ conditions, respectively; $\left.p<.011, .015 \& .082 ; p_{c}=.017, .025 \& .05\right)$. Analysis of variance of only the first measurements after smoking revealed that curve surface increased significantly after all THC doses, relative to placebo $\left(F_{1,14}=8.66,10.45 \& 4.96\right.$ for the 100,200 and $300 \mu \mathrm{g} / \mathrm{kg}$ conditions, respectively; $p<.011, .006 \& .043 ; p_{c}=.025, .017 \& .05$ ).

In summary, body sway was affected by all three THC doses, but this effect had dissipated three hours after smoking. Body sway parameters did not discriminate between different THC doses.

\section{Heart Rate}

ECG data could not be obtained from every subject in all conditions due to recurrent equipment failures. Though the problem was solved in time to obtain complete data from all 15 subjects in the highest dose conditions, analyses could only be conducted using data from 14 and 9 subjects in the middle- and low-dose conditions. The results of those analyses are summarized in Tables 5.8 and 5.9 for the standard and car following tests, respectively.

Both tables show that driving under the influence of THC is accompanied by shorter interbeat intervals (i.e. increased heart rates) and decreased variation both in the time domain (CV-IBI) and frequency domain between 0.07 and $0.14 \mathrm{~Hz}$ (PWR-HR). No clear dose-response relationship was found, except in mean IBI in the car following test.

With respect to the standard driving test, MANOVA showed that all THC doses produced significantly shorter IBIs relative to placebo $\left(F_{1,7}=9.07, F_{1,12}=7.63 \& F_{1,13}=21.61\right.$ for the 100 , 200 and $300 \mu \mathrm{g} / \mathrm{kg}$ conditions, respectively; $p<.02, .017 \& .001 ; p_{c}=.05, .025 \& .017$ ). CV-IBI was significantly diminished after the two higher doses $\left(\mathrm{F}_{1.12}=11.52 \& \mathrm{~F}_{1.13}=28.90\right.$ for the 200 and $300 \mu \mathrm{g} / \mathrm{kg}$ conditions, respectively; $\left.p<.005 \& .001 ; p_{c}=.025 \& .017\right)$, but not the lowest. PWR-HR, on the other hand, was significantly diminished by all THC doses $\left(\mathrm{F}_{1,7}=18.62\right.$ $F_{1.12}=12.20 \& F_{1,13}=28.47$ for the 100,200 and $300 \mu \mathrm{g} / \mathrm{kg}$ conditions, respectively; $p<.004$, 
$\left..004 \& .001 ; p_{c}=.025, .05 \& .017\right)$. Thus, the more specific measure, PWR-HR, which includes only one source of heart rate variability, is more sensitive to THC's effects than CV-IBI, a measure of total variability. In conclusion, both mean IBI and heart rate variability were significantly reduced by all THC doses. Since the latter measure is interpreted as a parameter of mental effort, it can be concluded that subjects needed to invest more effort in driving after smoking marijuana than after placebo.

Table 5.8 Mean interbeat interval time (IBI), coefficient of variation of IBI (CV-IBI), and power density of heart rate (PWR-HR) in the frequeney band between 0.07 and $0.14 \mathrm{~Hz}$ during the standard driving test.

\begin{tabular}{lllllll}
\hline & \multicolumn{2}{c}{$\mathrm{N}=9$} & \multicolumn{2}{c}{$\mathrm{N}=14$} & \multicolumn{2}{c}{$\mathrm{N}=15$} \\
& $0 \mu \mathrm{g} / \mathrm{kg}$ & $100 \mu \mathrm{g} / \mathrm{kg}$ & $0 \mu \mathrm{g} / \mathrm{kg}$ & $200 \mu \mathrm{g} / \mathrm{kg}$ & $0 \mu \mathrm{g} / \mathrm{kg}$ & $300 \mu \mathrm{g} / \mathrm{kg}$ \\
\hline IBI (ms) & 792 & 721 & 805 & 743 & 797 & 709 \\
CV-IBI (\%) & 5.97 & 4.91 & 5.40 & 4.48 & 5.41 & 4.15 \\
PWR-HR & 1114 & 666 & 877 & 570 & 862 & 499 \\
\hline
\end{tabular}

With respect to the car following tests, MANOVA showed that mean IBI decreased significantly after the two higher doses $\left(F_{1,9}=9.66 \& F_{1,7}=26.24\right.$ for the 200 and $300 \mu \mathrm{g} / \mathrm{kg}$ conditions, respectively; $\left.p<.013 \& .001 ; p_{c}=.025 \& .017\right)$, but not after the lowest. Mean IBI was always larger during the second ride relative to the first resulting in a significant Time effect in all conditions $\left(F_{1,9}=52.92, F_{1.9}=57.02 \& F_{1,7}=79.07\right.$ for the 100,200 and $300 \mu g / k g$ conditions, respectively; $\left.p<.001, .001 \& .001 ; p_{\mathrm{c}}=.05, .025 \& .017\right)$. Drug by Time interaction was not significant. This means that subjects were more relaxed when they performed the second than the first car following test, whether they had inhaled THC or placebo.

Table 5.9 Mean interbeat interval time (IBI), coefficient of variation of IBI (CV-IBI), and power density of heart rate (PWR-HR) in the frequency band between 0.07 and $0.14 \mathrm{~Hz}$ during the car following tests. Data from the first and second tests are separated by a slash.

\begin{tabular}{lllllll}
\hline & \multicolumn{2}{c}{$\mathrm{N}=9$} & \multicolumn{2}{c}{$\mathrm{N}=14$} & \multicolumn{2}{c}{$\mathrm{N}=15$} \\
& $0 \mu \mathrm{g} / \mathrm{kg}$ & $100 \mu \mathrm{g} / \mathrm{kg}$ & $0 \mu \mathrm{g} / \mathrm{kg}$ & $200 \mu \mathrm{g} / \mathrm{kg}$ & $0 \mu \mathrm{g} / \mathrm{kg}$ & $300 \mu \mathrm{g} / \mathrm{kg}$ \\
\hline IBI (ms) & $739 / 821$ & $697 / 801$ & $778 / 859$ & $697 / 790$ & $750 / 841$ & $646 / 755$ \\
CV-IBI (\%) & $5.48 / 6.31$ & $4.69 / 4.86$ & $5.18 / 5.83$ & $3.85 / 5.25$ & $4.67 / 5.41$ & $4.05 / 4.57$ \\
PWR-HR & $983 / 1347$ & $608 / 807$ & $909 / 1094$ & $438 / 967$ & $726 / 993$ & $405 / 633$ \\
\hline
\end{tabular}


CV-IBI was diminished after smoking THC in each condition relative to placebo and these effects were only nearly significant $\left(F_{1,9}=7.09, F_{1.9}=8.40 \& F_{1.7}=6.21\right.$ for the 100,200 and $300 \mu \mathrm{g} / \mathrm{kg}$ conditions, respectively; $\left.p<.026, .018 \& .041 ; p_{c}=.025, .017 \& .05\right)$. As in the standard test, PWR-HR was more sensitive to drug induced variability reduction; MANOVA showed that PWR-HR was significantly affected by all doses $\left(F_{1,9}=10.46, F_{1,9}=6.04\right.$ \& $F_{1.7}=9.01$ for the 100,200 and $300 \mu \mathrm{g} / \mathrm{kg}$ conditions, respectively; $p<.010, .036 \& .020$; $\left.p_{c}=.017, .05 \& .025\right)$. Though PWR-HR was generally higher in the second than in the first test, a significant Time effect was only found in the $200 \mu \mathrm{g} / \mathrm{kg}$ condition $\left(\mathrm{F}_{1,9}=8.58 ; p<.0168\right.$; * $\left.p_{c}=.017\right)$. Though subjects felt more relaxed in the second car following test than the first, as measured by IBI, both rides required much the same mental effort, as measured by heart rate variability.

\section{Intra-Subject Relations between Variables}

The average intra-subject correlation between [THC] and perceived "high" was, as in the previous study, very high $(\mathrm{R}=0.90 ; p<.01)$. The correlation between measured SDLP and [THC] measured before driving was $0.63(p<.05)$, individual correlations ranging from -.14 to .96; between SDLP and [THC] measured after driving $0.57(p<.05)$, individual correlations ranging from -.10 to .92 . These correlations closely resemble those found in the previous study where SDLP correlated 0.59 and 0.42 in the first and second tests, respectively. This means that the change in driving performance, as measured by SDLP in the standard driving test, is, within individuals, moderately related to the existing [THC]. Performance in the car following test was not significantly correlated with [THC].

The square root of the number of side contacts in the hand steadiness test was significantly correlated to [THC] and perceived "high" (both $r=.66 ; p<.01$ ). SDLP scores were significantly related to hand instability measured before driving $(r=.52 ; p<.05)$, but not with scores obtained after driving ( $r=.35 ; \mathrm{ns})$. None of the other laboratory performance measures were either related to plasma concentrations of the drug or to driving performance.

In summary, as [THC] varied within a given individual so did that individual's perceived "high", hand steadiness and SDLP or road tracking error in the standard test.

\section{Inter-Subject Relations between Variables}

Relationship between Drug Levels and Performance. Inter-subject correlational analysis between [THC] and [THC-COOH] on one hand and performance parameters on the other failed to reveal any consistent relationship. The most consistent, yet still not strong, relationships found were those involving [THC] on one hand and mean speed in the standard driving test and CTT performance in the laboratory on the other (Table 5.10). Correlations with Log [THC] were generally the same as with [THC]; those with [THC-COOH] were, except a few, generally lower than those with [THC].

The correlations presented in Table 5.10 were derived from raw scores. Another question is whether changes in performance from corresponding placebo levels were related to prevailing [THC]. Correlational analyses showed, however, that these correlations were even smaller than those with the raw scores. Thus, neither [THC] nor [THC-COOH] predicted performance or performance impairment. 
Table 5.10 Correlations between [THC] and raw performance scores in the marijuana conditions. If performance was measured only once (like SDLP), then the same scores were correlated with [THC] values as measured before and after the driving tests.

\begin{tabular}{lccccccc}
\hline & \multicolumn{2}{c}{$100 \mu \mathrm{g} / \mathrm{kg}$} & \multicolumn{2}{c}{$200 \mu \mathrm{g} / \mathrm{kg}$} & \multicolumn{2}{c}{$300 \mu \mathrm{g} / \mathrm{kg}$} \\
& $1^{\text {st }}$ & $2^{\text {nd }}$ & $1^{\text {st }}$ & $2^{\text {nd }}$ & $1^{\text {st }}$ & $2^{\text {nd }}$ \\
\hline Standard Driving Test: & & & & & & \\
SDLP & -.33 & .02 & -.13 & .09 & -.25 & -.18 \\
SP & -.26 & -.18 & -.50 & -.20 & $-.55^{*}$ & -.61 \\
SDSP & -.22 & -.14 & -.34 & -.32 & -.20 & -.15 \\
Car Following Test: & & & & - & &. \\
Adjusted RT & -.18 & .20 & .41 & .24 & .04 & .05 \\
Headway & .32 & .15 & .39 & -.02 & .18 & .05 \\
CV-Headway & -.21 & .40 & .27 & .43 & - & -.29 & -.09 \\
Laboratory Tests: & & - & & & & \\
$\lambda_{c}$ & $-63^{*}$ & .31 & .48 & .27 & .45 & .18 \\
Side Contacts & $.45^{*}$ & -.38 &. $.54^{*}$ & .32 & .44 & .50 \\
OC-O & -.25 & -.13 & -.43 & .02 & -.38 & .06 \\
OC-C & -.05 & -.02 & .36 & -.25 & -.26 & -.37 \\
\hline
\end{tabular}

Relationship between Driving Impairment and Willingness to Drive. In general, the average road tracking impairment in the standard test of those subjects, who indicated before the driving tests commenced that they would normally not have driven under the imagined circumstances, was greater than of those who would have driven. Statistically, these effects were neither significant when willingness to drive for "unimportant though gratifying" reasons was used as the grouping factor, nor when willingness to drive for "urgent" reasons was used. This was probably due to the small number of subjects who would not have driven under the former condition, and would have driven under the latter condition. When willingness to drive for "important, but avoidable" reasons was used as the grouping factor, no significant difference in road tracking impairment was found between the groups after the highest THC dose; but, following the two lower doses, subjects who would not have driven were significantly more impaired than those who would have driven $\left(F_{1,13}=10.38 \& 6.93\right.$ for the 100 and $200 \mu \mathrm{g} / \mathrm{kg}$ conditions, respectively; $\left.p<.007 \& .021 ; p_{c}=.017 \& .025\right)$.

Relationship between Driving Performance and Other Measures. In the previous study, SDLP was moderately correlated with $\lambda_{t}$ from the CTT. The present study showed the same pattern of correlations i.e. -.41 and -.50 in the $100 \mu \mathrm{g} / \mathrm{kg}$ condition, -.49 and -.37 in the $200 \mu \mathrm{g} / \mathrm{kg}$ condition, and $-.58(p<.05)$ and $-.54(p<.05)$ in the $300 \mu \mathrm{g} / \mathrm{kg}$ condition. Despite the interesting theoretical aspects of these consistent results, correlations of such magnitude are generally considered too low for predictive purposes. Performance in the car following test was not significantly correlated with $\lambda_{r}$. Neither hand steadiness nor body sway were related to performance in the standard driving test or the car following test. 
As in the previous study, repeated measures analysis of SDLP in the standard test was repeated with reported Frequency of cannabis smoking as a between-subjects factor. MANOVA failed to reveal any significant effect.

The relationship between test performance and previous experience of driving under the influence could not be determined: too few indicated having driven in that condition more than a few times (Table 5.1).

\section{DISCUSSION}

The conservative approach of administering THC according to an ascending, placebo controlled, dose series achieved its purpose of ensuring the subjects' safety. All 'were able to complete the series without suffering any untoward reaction while driving. However it is fair to ask whether this approach could have conceivably biased the results toward minimization of the drug's effects. We have three reasons for believing that this was not the case. Driving performance during successive repetitions of the standard highway driving test after placebo smoking was relatively constant; so that the reference for judging effects of every dose's effects was essentially the same. The measured mean changes in SDLP after the lowest and highest THC doses in the present study were about what they had been in the previous study where dose orders were counterbalanced. Finally, there was apparently one dose sequence effect on car following performance but it was the opposite of any which could bias the results toward minimizing THC's effects. Such a bias would have existed if the subjects choose to operate more cautiously as doses ascended. Yet as described below, they appeared to operate most cautiously in the car following test after the lowest dose. If any bias existed in this test it was toward maximizing, rather than minimizing THC effects.

Road tracking performance in the standard test was impaired in a dose-related manner by THC. The $100 \mu \mathrm{g} / \mathrm{kg}$ dose produced a slight elevation in mean SDLP $(1.1 \mathrm{~cm})$, albeit nearly significant. The $200 \mu \mathrm{g} / \mathrm{kg}$ dose produced a significant elevation $(1.8 \mathrm{~cm})$, of dubious practical relevance. The $300 \mu \mathrm{g} / \mathrm{kg}$ dose produced a highly significant elevation $(2.9 \mathrm{~cm})$ which may be viewed as practically relevant but unexceptional in comparison with similarly measured effects of many medicinal drugs.

For example, diazepam given for one week in its lowest therapeutic dose ( $5 \mathrm{mg}$, thrice daily) caused anxious patients to drive with a mean SDLP about $7 \mathrm{~cm}$ higher than their premedication baseline (Van Laar et al., 1992). Furthermore, THC's effects on SDLP were, after the 100, 200 and $300 \mu \mathrm{g} / \mathrm{kg}$ doses in this study, about the same as those of BACs $=0.02,0.04$ and $0.06 \mathrm{~g} \%$ according to the empirical equation described by Louwerens et al. (1985, 1987). The comparison reinforces our impression of the relevance of SDLP changes after THC. The two lower doses produced elevations less than the lowest BAC associated with intoxication and an elevated risk of causing a traffic accident (i.e. $0.05 \mathrm{~g} \%$; Borkenstein et al., 1964; Council on Scientific Affairs, 1985), whereas the highest dose, and one preferred by the drug's users, produced a marginally greater elevation. But even this change in SDLP can not, by itself, be taken to indicate exceptional impairment.

It is often reported that subjects compensate for THC's adverse effects on driving abilities by operating at slower than normal speeds, especially through curves or slaloms. Our subjects were instructed to maintain a speed of $95 \mathrm{~km} / \mathrm{h}(58 \mathrm{mph})$ unless compelled to slow down for safety reasons. Following marijuana smoking they drove with an average speed that was only 
slightly lower (maximum, $1 \mathrm{~km} / \mathrm{h}$ or $0.6 \mathrm{mph}$ ) than after placebo and very close to the prescribed level. They apparently felt that it was within their capability to safely perform this relatively simple task while operating at the normal highway speed.

The objective of confirming results obtained in the previous closed highway study was achieved in this one. It should be recalled that subjects' SDLPs were measured twice on the former occasion; i.e. in $22 \mathrm{~km}$ (13.7 mi) trials beginning 40 and 100 minutes after the initiation of smoking. The timing of the second trial most closely corresponded to that in the present study so their respective results will serve as the basis for comparison. Mean SDLP elevations after the lowest and highest doses differed little between the two studies: $100 \mu \mathrm{g} / \mathrm{kg}, 1.4$ and $1.1 \mathrm{~cm}$; and $300 \mu \mathrm{g} / \mathrm{kg}, 2.4$ and $2.9 \mathrm{~cm}$, respectively. Mean SDLP elevations after the $200 \mu \mathrm{g} / \mathrm{kg}$ dose differed somewhat between studies, being $3.3 \mathrm{~cm}$ in the first case and 1.8 in the second. Yet the results of the first study were anomalous in two respects. Not only did the former group's reaction in the $2^{\text {nd }}$ trial exceed that in the $1^{\text {st }}$ trial following the $200 \mu \mathrm{g} / \mathrm{kg}$ dose, it was also greater than their $2^{\text {nd }}$.trial reaction after the $300 \mu \mathrm{g} / \mathrm{kg}$ dose. Both results were in contradiction to plasma THC concentrations measured at these times. Thus the peculiar elevation in mean SDLP during the $2^{\text {nd }}$ trial after the intermediate dose was probably a consequence of sampling error. That it was not replicated in the present study should dispel any notions to the contrary.

The car following test was implemented for the. first time in the present study. In it, subjects maintained a headway of $45-50 \mathrm{~m}$ (148-164 ft) while driving in the successive placebo conditions. They lengthened mean headway by 8,6 and $2 \mathrm{~m}(26.2,19.7$ and $6.6 \mathrm{ft})$ in the corresponding THC conditions after 100,200 and $300 \mu \mathrm{g} / \mathrm{kg}$, respectively. The initially large drug-placebo difference and its subsequent decline is a surprising result in need of an explanation. If one considers that changes in headway after THC results from impaired distance perception, the inverse relationship between administered dose and mean headway defies explanation. But if one considers these differences as the results of the subjects' caution in approaching the task under the influence of THC on successive occasions, another explanation seems plausible.

Performing the car following test in the company of investigators while under the influence of THC was a novel experience for all subjects. Neither the investigators nor the subjects could predict how the latter would be able to operate the vehicle. The former were somewhat apprehensive and it would not be surprising if the latter were also, particularly, on the first occasion the test was performed after THC inhalation. Subjects were required to match their vehicle's speed with that of the preceding vehicle's and so were unable to reduce velocity as a compensatory action. They could however maintain a longer headway and thereby slightly increase their margin of safety which might be needed if THC retarded their reactions. We believe they did so after receiving the lowest THC dose. When nothing untoward happened and the subjects' confidence in their ability to control the vehicle grew, they apparently tended to diminish headway after each of the succeeding doses so that it came progressively closer to the distance measured after all of the placebo treatments. Thus our explanation for the initial difference between headways maintained after THC and placebo, and why it diminished in subsequent pairs of these conditions, is that the subjects' caution was greatest the first time they undertook the test under the influence of THC and progressively less thereafter.

The only other plausible explanation is that lower THC doses induce a greater sense of caution than higher doses. There is of course no way to determine which of the two explanations is valid from the results of this study. But the fact that the subjects reported feeling less calm and content as the doses increased seems to contradict the notion that they simultaneously 
became less cautious. Whatever explanation is favored, it is clear that large doses of THC have little effect on mean headway during car following.

Reaction time to changes in the preceding vehicle's speed increased following THC treatment, relative to placebo. The administered THC dose was inversely related to the change in reaction time, as it was to headway. Mean increases in reaction time were $0.55,0.41$ and $0.19 \mathrm{~s}$ following the 100,200 and $300 \mu \mathrm{g} / \mathrm{kg}$ dose, respectively. However, reaction time data were confounded with headway. That is, increased reaction times were partly due to longer headway. Statistical adjustment for this confounding resulted in smaller and non-significant increases in reaction time following marijuana treatment, the greatest impairment $(0.32 \mathrm{~s})$ being observed in the first test following the lowest THC dose. Headway variability followed a similar pattern as mean headway and reaction time; the greatest impairment was found following the lowest dose.

A secondary objective of the study was to determine whether degrees of impairment would correlate between the two tests in a manner indicating a general influence of THC on driving behavior. The results obtained in this study showed that this was not the case; a significant doserelated impairment was found with road tracking, but not with car following. Test duration can not have been the critical point, since the standard test in the previous study was of the same duration as the present car following test.

The car following test was both less sensitive and reliable than it could be following the removal of certain procedural flaws. Intervals between successive maneuvers were practically constant and the preceding vehicle's deceleration was both abrupt an stereotyped. Thus the occurrence and nature of the maneuvers were highly predictable for the subjects.

Greater irregularity in both the timing of maneuvers and the profile of velocity changes would probably increase the sensitivity of the test. After the fact we recognized that the indirect and discontinuous method used for measuring headway produced a degree of error variance which appreciably reduced the reliability of these data. Equipment has become available since the initiation of this study which now makes it possible to measure headway directly and continuously (i.e. an inexpensive pulsed laser reflection recording system). Use of this equipment should increase the reliability of headway measurement in future applications of this test. Finally, the greatest source of error variance was the procedure of allowing the subject to assume, within limits, whatever headway he/she chooses. Whether this is a flaw or not depends upon one's desire to measure or control headway at the beginning of maneuvers. However the subject's choice of headway is certainly a factor which inflates reaction time error variance. Adjusting the data statistically to overcome the confounding effect of headway on reaction time is only a partial solution to the problem. We would probably have been wiser to strictly enforce headway control in order to increase the reliability of reaction time measurements and their sensitivity to drug effects.

Without trying to minimize the impact of procedural errors on the data, it is doubtful whether any flaw or all in combination seriously obscured a practically important THC effect. SDLP recorded in the standard highway driving test was about as sensitive to low-dose THC effects as any of the traditional laboratory performance measures taken in the study. Moreover the average intra-subject correlation between [THC] and SDLP was as high or higher than any measured between the drug's plasma concentration and another performance variable. This relationship could not have been measured if SDLP were not one of the most sensitive parameters known for measuring the effects of THC. The fact that car following performance 
was less sensitive does not mean that it would have failed to reveal effects of practical importance if these truly existed. SDLP showed no such effects even after the highest dose.

The reasons why SDLP and not car following performance showed the modest impairing effects of THC may have less to do with the inadequacy of the latter test than to the difference between what the two tests measure. SDLP is controlled by a very fast and high capacity human information processing system which operates in a wholly "automatic" manner. That is, outside of conscious control. The process is relatively impervious to environmental changes as shown again by the high reliability of SDLP under repeated placebo conditions in the present study. It is, however, highly vulnerable to internal factors that retard the flow of information through the system. THC and other drugs are among these factors. When they interfere with the process to elevate SDLP there is very little the afflicted individual can do in way of compensation. Car following performance on the other hand depends upon more discrete perception of events leading to a conscious decision, a response selection and its execution. Performing the test involves far more sustained attention and conscious effort than does road tracking in the standard test. Because car following performance is under conscious control and well within the speed limitations of "cognitive" compensatory mechanisms it is possible for individuals to recognize their deficiencies and correct them by effort that increases attention. In short, any deficiencies that THC might have otherwise produced may have been overcome by the subjects' compensatory effort. The cost of effort focused on accomplishing a task is however accompanied by less capacity left for performing another in parallel. The subjects indeed related that their investment of effort in the first car following test increased with the administered dose, and relative to corresponding placebo levels, more so than in the standard driving test. Though these differences were not statistically significant in either case, they were in line with the significant reduction in heart rate variability, that occurred independently of mean rate changes after every THC dose, including the lowest. Together the findings support the premise that THC increases the requirement for compensatory effort during car following which maintains constant performance, but possibly reduces the capacity for undertaking any. activity in parallel. Coupled with THC's reputably adverse effect on the ability to divide attention between tasks performed simultaneously (Smiley, 1986), the net effect might constitute a more serious impediment to safe driving than any observed in this study. It will be interesting to explore this possibility in further research.

Subjects' report of their willingness to drive under specified conditions of different urgencies were similar to those in the previous study. The lower the administered THC dose, and the more urgent the reason for driving, the more subjects declared that they would be willing to drive. Furthermore, there was a tendency for subjects who would normally not have driven to be more impaired in road tracking than those who would. Apparently these subjects recognized their respective degrees of impairment while under the influence of every THC dose. This is supported by the subjects' judgments of their own driving quality which changed in a realistic dose related manner after marijuana smoking.

Critical tracking and hand steadiness tests were more sensitive to THC induced impairment than in the previous study. This confirms our impression that the earlier CTT version was poorly conceived. It also indicates that applying the correct procedures for measuring hand steadiness is very important; i.e. subjects should not be allowed to rest their hand on the table while performing the test. Both tests showed dose-related impairment shorty after cessation of smoking. When the tests were repeated three hours later, hand instability was still present though to a lesser degree. The effect of the highest but not the two lower doses on CTT performance persisted undiminished from the first to the second test, or over a 3-hour period after smoking. 
These results partially confirm results obtained by Sharma and Moskowitz (1975). They found that THC $200 \mu \mathrm{g} / \mathrm{kg}$ had a virtually undiminished effect on tracking performance for up to four hours. The time course of postural instability after marijuana smoking followed a different profile. All THC doses increased body sway to the same extent shortly after cessation of smoking but none did three hours later.

An important practical objective of this study was to determine whether degrees of driving impairment can be accurately predicted from either measured concentrations of THC in plasma or performance measured in potential roadside "sobriety" tests of tracking ability or hand and posture stability. These results, like many reported before, indicate that none of these measures accurately predicts changes in actual driving performance under the influence of THC. CTT performance came closest but even its correlation with driving was only $r=.50$. However, that test might well be included in a battery of similarly predictive tests, measuring different abilities, to collectively yield a single more predictive index of impairment. 


\section{CHAPTER 6 - MARIJUANA, ALCOHOL AND URBAN CITY DRIVING}

\section{INTRODUCTION}

In the previous study, THC doses of $200 \mu \mathrm{g} / \mathrm{kg}$, and higher, produced significant impairment of road tracking, but not car following, performance. The lowest dose, $100 \mu \mathrm{g} / \mathrm{kg}$, failed to produce significant effects on road tracking, but increased headway during car following. The latter observation was interpreted as the result of increased caution, since mean headway did not change following subsequent higher THC doses. It therefore seems that low doses of THC do not, or only slightly, impair driving performance. Yet normal driving is far more complex and varied than simply maintaining a safe lateral position and headway during uninterrupted travel on a highway. A THC dose having no effect on these parameters might still impair driving performance in more complex urban driving situations.

There were logical and safety reasons for restricting the THC dose in the third driving study to that which had failed to produce significant impairment in the second. Both the 200 and $300 \mu \mathrm{g} / \mathrm{kg}$ doses impaired performance on the highway and could be expected to do so again in the urban driving environment. There the consequences of high dose THC effects are more difficult to predict and therefore safely control. The $100 \mu \mathrm{g} / \mathrm{kg}$ dose had some significant effects on the highway but none that could rightfully be called dangerous. This dose might still cause impairment in more complex city driving, but the risk was judged to lie within the realm of the acceptable. For that reason it was given to a group of regular cannabis users, along with placebo in the present study.

For comparative purposes another group of regular alcohol users were treated with a modest dose of their preferred recreational drug, and again placebo, before undertaking the same test. It was hoped that this addition would not only verify the sensitivity of the test but also allow a comparison between effects of recreational drugs that Dutch society considers as illicit and licit when both are given in relatively low doses. The comparison was not designed to show that one drug is "safer" for use by drivers than the other. Surely neither are safe when consumed before driving in high doses. However if respective low dose effects are comparable then one would be justified to conclude that THC may be considered as posing a traffic safety hazard which is in some respects similar to alcohol's.

The only study that has been conducted in actual traffic before this program started was also a city driving study. Klonoff (1974) assessed the effects of two THC doses, 4.9 and $8.4 \mathrm{mg}$ THC which are equivalent to 70 and $120 \mu \mathrm{g} / \mathrm{kg}$ for a $70 \mathrm{~kg}$ (154 lb) person. Aspects of subjects' driving performance were scored by a professional examiner using an abbreviated version of the British Columbia Department of Motor Vehicles' standard driver's licencing test. The results showed that subjects performed less competently when under the influence of the highest, but not the lowest, dose. In particular, they scored lower on judgment and concentration scales. Moskowitz (1985) and Smiley (1986) criticized the method of measuring driving performance on the grounds that the examiners' reliability was never determined and that the scoring instrument had never been shown to. provide measures related to driving safety. Smiley questioned, for example, whether ratings of posture and irritability are relevant for good driving performance. These are sound criticisms but one has to assume that Klonoff's approach should 
have been sensitive to serious driving performance impairment, related to safety, if it had in fact occurred.

Two scoring methods were employed in the present study. The first was in fact a method similar to that applied by Klonoff; i.e. the driving instructor acting as the safety controller during the tests retrospectively rated the driver's performance using a standard scale. This method has been applied previously to show the impairing effects of alcohol (De Gier, 1979) and diazepam (De Gier et al., 1981) in similar situations. Jones (1978) criticized this use of driver licensing assessment procedures. She opposed the lack of precise definitions for many of the behaviors rated by examiners and the requirement for rating all of them at once. In contrast to this "molar" approach, she developed a more "molecular" one for evaluating driving proficiency. Her method was also applied in the present study. It involves the employment of specially trained observers who apply simple and strict criteria for recording when the driver makes or fails to make each in a series of observable responses at predetermined points along a chosen route.

The professional observer's global ratings are inherently less reliable than the scores obtained by the molecular rating scheme. Still the molar approach has some advantages. The professional's experience with many drivers operating in all traffic situations provides him with the ability to integrate far more information than is possible to obtain from limited performance sampling. He has internalized a broad concept of acceptable driving performance and applies more flexible criteria for judging when it is unsafe within a particular test situation. Of course the danger that a professional's biases may influence his judgments needs to be overcome by training and his adherence to structured rules which are specific for the investigation. But when this is done, he may provide a more valid estimation of the overall safety of a subject's driving performance. If this were not the case it would be difficult to explain how every developed society relies upon the professional's and not a traffic scientist's opinion of whether a particular individual should be licensed to drive.

The objective of this study would be satisfied in one way if neither observer rating method yielded a significant difference between driving performance after $100 \mu \mathrm{g} / \mathrm{kg}$ THC and placebo. These results would confirm those obtained in the previous study by indicating that the selected dose lies below that capable of impairing driving performance. This.conclusion would only be warranted, however, if it could be shown that the tests were sensitive enough to measure significant driving performance impairment after alcohol relative to placebo. If that were not the case, test insensitivity could be judged as the factor responsible for negative results, rather than the lack of a THC effect.

The objective would be satisfied in another way if either or both rating methods showed significant impairment after THC. Such results would indicate that any dose likely to be consumed before driving should be considered hazardous, regardless of whether alcohol's effects were the same, more or less. In the event that significant impairment occurred after THC, we were prepared to determine its relationship with plasma concentrations of THC and THC-COOH measured at about the same time. 


\section{METHODS}

\section{Subjects}

Two groups of sixteen new subjects apiece, equally comprised of men and women, participated in the study. The groups will be referred to by the alcohol and marijuana group. Subjects in both groups were recruited according to the same inclusion/exclusion criteria as before with one exception. Subjects in the alcohol group were regular users of alcohol but not marijuana (see below). All subjects were individually trained to perform the city driving test in a preliminary "dress rehearsal" and were familiarized with the other tests and questionnaires.

Plasma analyses after conclusion of the study showed that two males' plasma contained neither THC nor THC-COOH in any sample. It was concluded that these subjects had not inhaled smoke so their data were excluded from further analyses.

Characteristics of the remaining subjects are given in Table 6.1. Except for the reported incidence of marijuana use, there were no significant differences between any of the groups' characteristics.

Table 6.1 Mean \pm SD (range) of subjects' characteristics.

\begin{tabular}{lrr}
\hline & Alcohol Group & Marijuana Group \\
\hline $\mathrm{N}$ & 16 & 14 \\
Age (yrs) & $23.7 \pm 2.7(20-28)$ & $22.4 \pm 3.5(20-34)$ \\
Weight (kg) & $68.1 \pm 7.9(60.0-86.1)$ & $67.9 \pm 8.9(54.0-90.5)$ \\
Weight.(lb) & $150.1 \pm 17.4(132.2-189.8)$ & $149.7 \pm 19.6(119.0-199.5)$ \\
\# Alcoholic Drinks/Week & $8.3 \pm 6.2(1-21)$ & $10.6 \pm 8.4(1-30)$ \\
Smoking Experience (yrs) & 0 & $3.1 \pm 2.1(1-10)$ \\
\# Joints/Month & 0 & $2.3 \pm 2.5(1-8)$ \\
Driving Experience (yrs) & $-12.8 \pm 2.6(2-10)$ & $4.1 \pm 3.3(2-15)$ \\
Driving Experience (km x 1000) & $35.3 \pm 28.3(5-90)$ & $28.6 \pm 45.7(6-180)$ \\
Driving Experience (mi x 1000) & $21.9 \pm 17.6(3-56)$ & $17.8 \pm 28.4(4-112)$ \\
\hline
\end{tabular}

\section{Design, Doses and Administration}

The study was conducted according to a mixed between-groups within-subjects design, one group drinking alcohol and placebo alcohol and the other smoking marijuana and placebo marijuana.

Alcohol was administered as $99.8 \%$ ethanol mixed with orange juice and Grand Marnier essence to a volume of $250 \mathrm{ml}$. The dose was $0.43 \mathrm{~g} / \mathrm{kg}$ lean body mass (On average, this resulted in a dose of $0.36 \mathrm{~g} / \mathrm{kg}$ body weight in males, and $0.31 \mathrm{~g} / \mathrm{kg}$ in females). Lean body mass was calculated by subtracting the percentage of fat, determined by skinfold thickness (Durnin and Womersley (1974), from total body weight. The dose was chosen to yield a Blood Alcohol Concentration (BAC) approaching $0.05 \mathrm{~g} \%$ when the driving test commenced 45 minutes after onset of drinking. Subjects were instructed to fast $21 \frac{1}{2}$ hours before drinking and to ingest the dose within 5 minutes. Alcohol in the subjects' expired air was monitored using aLion S-D3.Breath-Alcohol Analyzer to ensure that subjects would not drive with BACs that were higher than $0.05 \mathrm{~g} \%$, which is the legal limit in The Netherlands. 
Marijuana and placebo cigarettes were obtained from the same source as before. The subjects were treated on separate occasions with THC doses of 0 and $100 \mu \mathrm{g} / \mathrm{kg}$. Placebo cigarettes were prepared by ethanol extraction of THC from the plant stock. Marijuana cigarettes were prepared from batches containing $1.77 \%$ THC. Cigarettes were cut to different lengths to provide the doses appropriate for the individuals' body weights. Cigarettes appeared identical in both treatment conditions and were smoked through a plastic holder in a fashion determined by the subject but with the constraint that smoking had to be finished within five minutes. After cessation of smoking, cigarettes were retained for subsequent gravimetric estimation of THC consumed. These analyses revealed that the average ( $\pm S D$ ) amount of consumed THC in the marijuana condition was $6.9( \pm 1.0) \mathrm{mg}$ or $101( \pm 6) \mu \mathrm{g} / \mathrm{kg}$.

Half of both groups received the treatments in the same order, the others, in reverse order. The driving instructor and observer who rated subjects' driving performance were blind regarding both the administered drug (active or placebo) and the subject's group membership.

\section{Testing Procedures}

Upon arrival at the laboratory, subjects were tested for the presence of alcohol in breath. Subjects in the marijuana group were further tested for the presence of cannabinoids and other drugs in urine (as described in Chapter 2). If cannabinoids were found, a blood sample was taken for later verification of the presence of THC. The schedule of further activities on -testdays is shown in Table 6.2.

Table 6.2 Schedule of activities on test-days.

\begin{tabular}{lcr}
\hline $\begin{array}{l}\text { Alcohol Group } \\
\text { Relative Time (min) }\end{array}$ & Activity & \multicolumn{2}{c}{ Marijuana Group } \\
\hline $0-5$ & Relative Time (min) \\
$30-35$ & $\begin{array}{c}\text { Hand Steadiness and } \\
\text { Time Perception Tests }\end{array}$ & $0-5$ \\
$35-40$ & $\begin{array}{c}\text { Blood Sampling and } \\
\text { Questionnaires }\end{array}$ & $15-20$ \\
$45-95$ & $\begin{array}{c}\text { City Driving Test } \\
95-100\end{array}$ & $20-25$ \\
& $\begin{array}{c}\text { Blood Sampling and } \\
\text { Questionnaires }\end{array}$ & $30-80$ \\
$105-110$ & $\begin{array}{c}\text { Hand Steadiness and } \\
\text { Time Perception Tests }\end{array}$ & $80-85$ \\
\hline
\end{tabular}




\section{Driving Test and Scaring Methods}

Driving tests were conducted in daylight over a constant $17.5 \mathrm{~km}(10.9 \mathrm{mi})$ route within the city limits of Maastricht (population ca. 115,000). The route was constructed through business and residential areas on 2-lane undivided streets and included a $6 \mathrm{~km}$ ( $3.7 \mathrm{mi})$ 4-lane divided segment on a major cross-city thoroughfare (Figure 6.1). Subjects drove their placebo and active drug rides through heavy, medium and low density traffic on the same day of the week, and at the same time of day. Maneuvers included left and right turns at some intersections and driving through others, left and right lane changes, and responding to traffic control devices (i.e. stop signs and signals). There was one special maneuver, repeated twice; i.e. executing a Y-turn on a residential street. A schematic representation of the standard route is shown in Figure 6.1 and the symbols used for maneuvers and road densities are shown in Table 6.3 (the performance variables will be discussed below).

Driving tests were conducted in a dual control 2-door sedan (Volkswagen Golf) normally used for driving instruction/examination. Two persons accompanied the subject: a licensed driving instructor sitting in the front passenger's seat and an trained observer sitting in the center of the rear seat. The former had access to redundant controls and his primary responsibility were controlling safety and giving the route instructions; and, to rate the driver's performance retrospectively (below) after the ride. The observer in the rear seat scored the driver's performance "on-line" and timed the duration of the ride.

As described above, two different procedures were employed for determining a driver's proficiency from observer ratings; i.e. the molecular and the molar methods. The major difference between both methods is that performance variables are repeatedly measured on-line at different points along the route using the molecular approach; and, only once, retrospectively, in the molar approach.

The molecular approach was adopted from Jones (1978) who simplified the scoring of driving behavior so that the trained observer, sitting in the rear seat, attends to only one event at a time. The events observed at specific points along the route are only few aspects of total driving behavior. Thus, the observer is enabled to attend completely to the occurrence of each event in sequence while ignoring all other behavior which would in any case vary between tests. All aspects of a maneuver, such as a left turn, will be scored a number of times over the course of the whole route, but never more than a single aspect at a given moment.

Jones defined twelve aspects, called performance variables (Table 6.3), which were repeatedly scored, 156 times in total, at specific points along the route. The route was designed to fulfill Jones' requirements concerning maneuvers and road densities. The scoring sheet consisted of the schematic route map with symbols indicating the driver behavior to be scored at each point (reproduced in Figure 6.1. with symbology defined in Table 6.3). The observer moved his finger along the route and attended only to the behavior indicated, comparing it with a predefined standard of performance, and circled (if correct), crossed (if wrong) or underlined (if not observed) the symbol. If, for example, the observer were to score Path (P) at a lane change $(\not)$ the correct response was defined as: 1 . maintains straight path while scanning, 2. signals before the lane change, 3 . steers smoothly with gradual angular movement, 4 . straightens vehicle in new lane, and 5 . cancels signal. If the driver failed to make one or more of these responses Path was scored as fail, otherwise pass. A complete description of all maneuvers is included in Appendix B. 


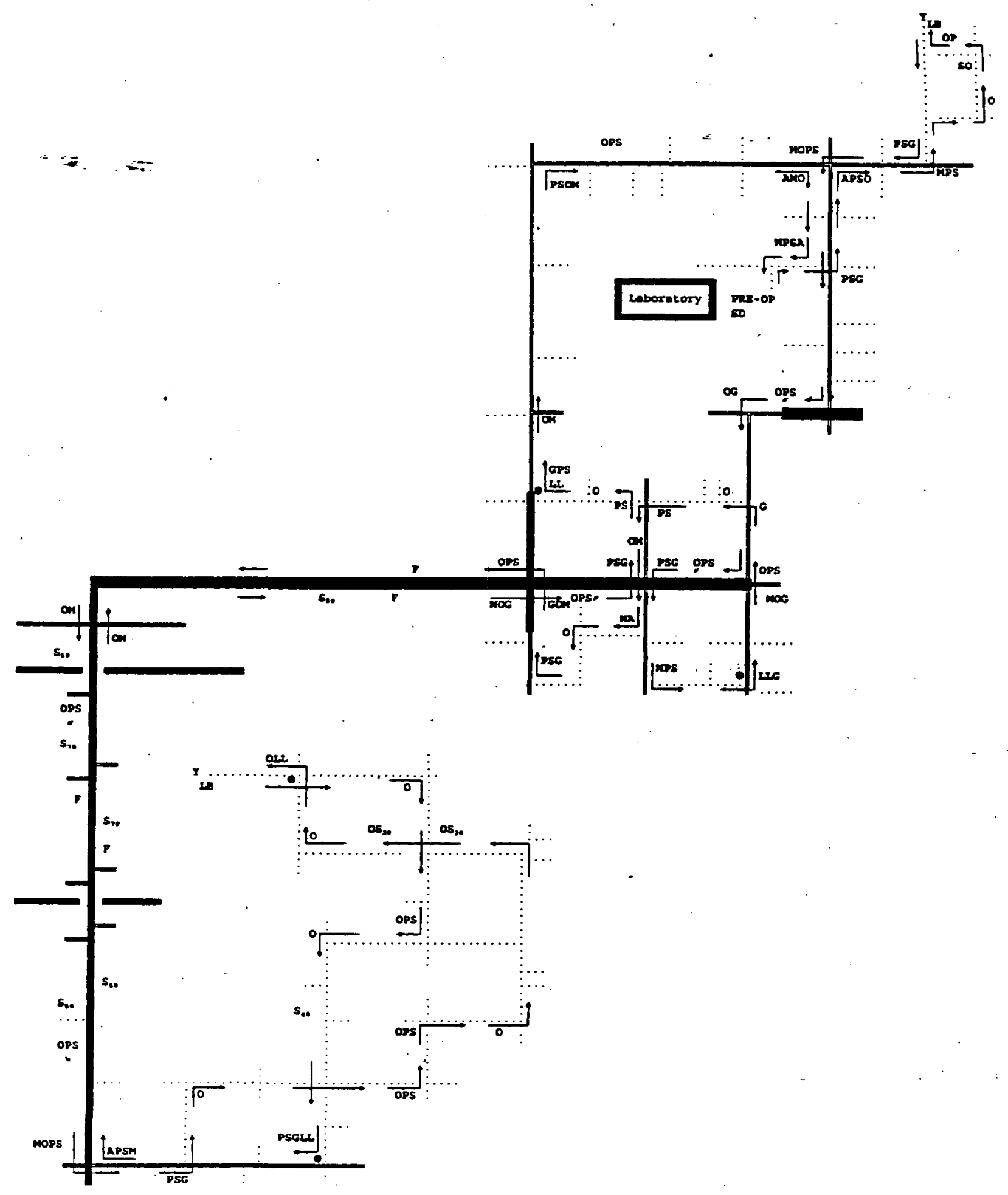

Figure 6.1 Scoring sheet for molecular approach, consisting of the schematic route map with symbols indicating the driver behavior to be scored at each point (for explanation of the symbols, see Table 6.3). 
Table 6.3 Maneuvers, road densities, performance variables and their symbols.

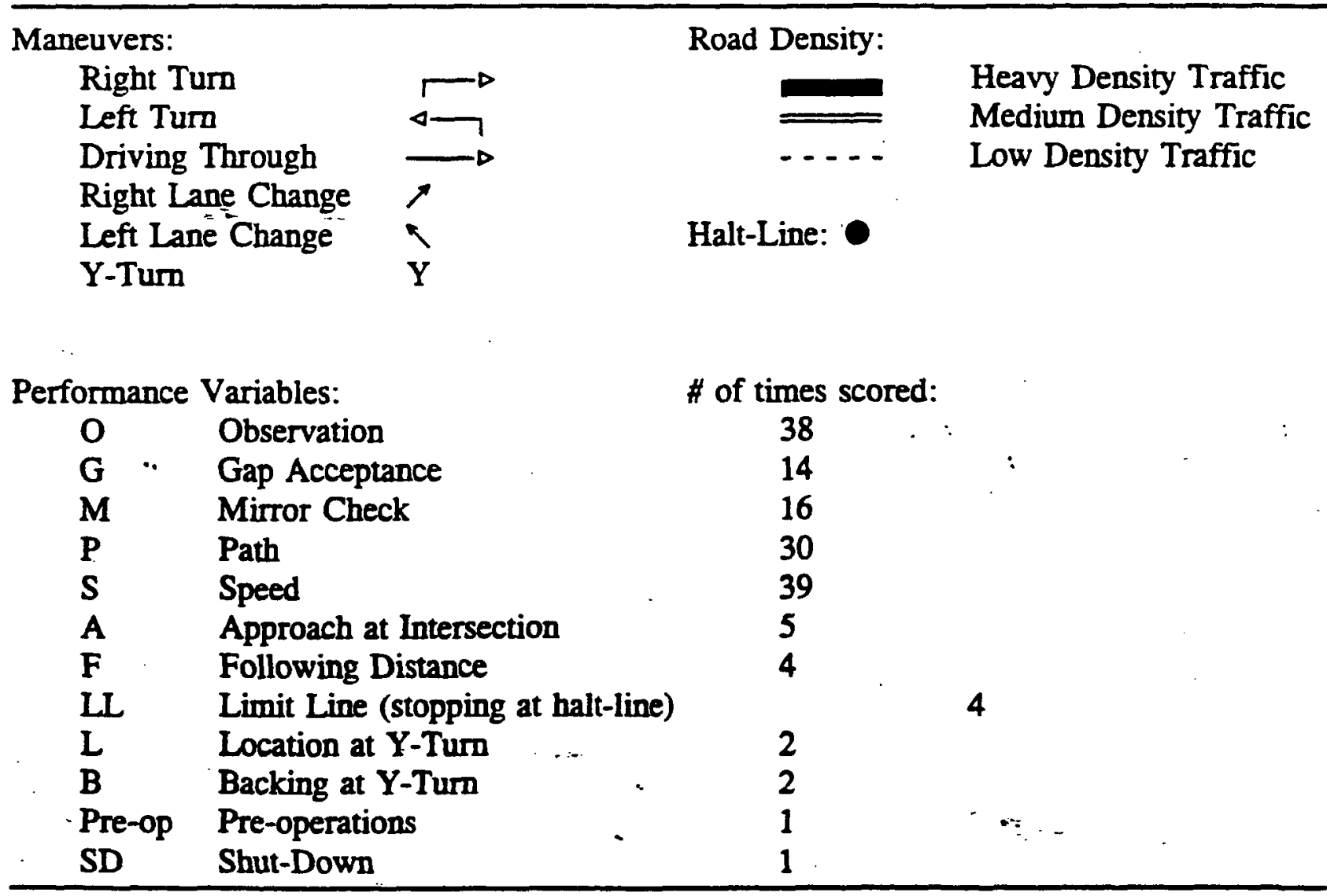

At the end of the test, the Total Score was calculated by summing all passing items and dividing by the total number of observed items. Subscores were also calculated for Observing, Car Control, Judgment and Other. (Table 6.4). Three additional scores were obtained from the driving instructor; 1. Instructor Control, the number of times the instructor took control, either orally or physically, 2. Hazard Score, the number of times the driver responded correctly to hazards divided by the total number of hazards encountered, and 3. Instructions, the number of times the driving instructor had to repeat a route instruction. All scores, except Instructor Control and Instructions, were expressed as per cent correct.

The molar approach required the driving instructor to retrospectively rate the driver's performance using a shortened version of the Royal Dutch Tourist Association's (ANWB) Driving Proficiency Test (Appendix B). This instrument is normally applied for practical purposes; e.g. remedial training, driver's licensing, qualifying for a reduction in insurance premiums, etc. Items that did not apply to the driving test, e.g. those regarding railway crossing and special maneuvers like driving backwards and parking, were dropped from the normal list. In total, 108 items were dichotomously scored, as either pass or fail. Total test performance was measured by the percentage items scored as "pass". Subscores were calculated for Vehicle Checks, Handling of Vehicle, Action in Traffic, Observation and Understanding of Traffic, and Turning (Table 6.5). 
Table 6.4 Dependent variables, and their operationalization, measured by the molecular approach.

\begin{tabular}{|c|c|}
\hline Dependent Variable & Operationalization \\
\hline Total Score & $\%$ correct of all 156 items \\
\hline Observing & $\begin{array}{l}\% \text { correct of } 68 \text { items comprising Observation, Gap Acceptance, } \\
\text { and Mirror Check }\end{array}$ \\
\hline $\begin{array}{l}\text { Car Control } \\
\text { Judgment }\end{array}$ & $\begin{array}{l}\text { \% correct of } 69 \text { items comprising Path and Speed } \\
\text { \% correct of } 13 \text { items comprising Approach, Following Distance } \\
\text { and Limit Line }\end{array}$ \\
\hline Other & $\begin{array}{l}\text { \% correct of } 6 \text { items comprising Y-turn Location and Backing, } \\
\text { Pre-Operations and Shut-Down }\end{array}$ \\
\hline $\begin{array}{l}\text { Hazard Score - } \\
\text { Instructor Control } \\
\text { Instructions }\end{array}$ & $\begin{array}{l}\text { \% of correct responses to hazards encountered along the route } \\
\text { \# of times instructor took control, either orally or physically } \\
\text { \# of times the driving instructor had to repeat a route instruction }\end{array}$ \\
\hline
\end{tabular}

Table 6.5 Dependent variables, and their operationalization, measured by the molar approach.

\begin{tabular}{|c|c|}
\hline Dependent Variable & Operationalization \\
\hline Total Score. & $\%$ correct of all 108 items \\
\hline Vehicle Checks & $\begin{array}{l}\text { \% correct of } 13 \text { items regarding preparations for driving and } \\
\text { driving away }\end{array}$ \\
\hline Handling of Vehicle & $\begin{array}{l}\text { \% correct of } 23 \text { items regarding posture and steering, controls } \\
\text { handling, speed adjustment and stopping, and driving through } \\
\text { curves }\end{array}$ \\
\hline Action in Traffic & $\begin{array}{l}\text { \% correct of } 60 \text { items regarding straight driving, behavior near } \\
\text { and at intersections, right turns, left turns, overtaking, driving in } \\
\text { lanes and lane changing, driving through traffic circles, and on } \\
\text { highways }\end{array}$ \\
\hline $\begin{array}{l}\text { Observation and Under- } \\
\text { standing of Traffic }\end{array}$ & $\%$ correct of 8 items regarding perception and traffic insight \\
\hline Turning & \% correct of 4 items regarding Y-turns \\
\hline Driving Time & time duration (s) of ride \\
\hline
\end{tabular}

\section{Questionnaires}

Questionnaires used in the previous studies were administered to the subjects in the present study. Subjective feelings of intoxication ("high" or "drunkness"), present cognitive and 
emotional state, and subjects' willingness to drive were assessed before the onset and after the conclusion of the driving tests. Subjects were also asked about their perception of the administered treatment, whether it was an active or placebo. At the end of each driving test, subjects were required to retrospectively rate the effort given in performing the test and perceived driving quality. Questionnaires are enclosed in Appendix A.

\section{Laboratory Tests}

Subjects performed two laboratory tests before and after driving. The hand steadiness test was used since it had shown a significant effect of the $100 \mu \mathrm{g} / \mathrm{kg}$ THC dose in the previous study and confirmation of this result would indicate the equivalent sensitivities of the present and previous groups to the drug. In addition a time perception test (method of interval production; Fraisse, 1963) was used to satisfy the investigators' curiosity about an oft-reported effect of THC. The former followed the same procedures as described in Chapter 5. The latter required the subjects to stand with eyes closed and indicate when he/she thought that 30 seconds had elapsed since a starting signal. The verbal response was timed to the nearest second by stopwatch.

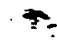

\section{Blood Sampling}

Blood samples were obtained by venepuncture immediately prior to and following all placebo and drug driving tests. Two aliquots containing $10 \mathrm{ml}$ each were heparinized and centrifuged, and the plasma fractions were placed in frozen storage for later assays to determine [THC] and $^{\text {" }}$ [THC-COOH], in the marijuana group; and, [EtOH], in the alcohol group. The analytical procedures regarding THC and THC-COOH assays were the same as in previous studies. As before, samples obtained in conjunction with placebo marijuana tests were only analyzed if the urine test had been positive. Plasma samples obtained from subjects in the alcohol condition were analyzed using gas-chromatography.

\section{Data Analysis}

For each variable measured on interval or ratio level, except plasma concentrations, change scores were computed by subtracting raw scores obtained in the placebo condition from those in the drug condition. In the figures, the mean change of the variable is depicted by the height of the bar and its standard error (SED) by the height of the vertical line above or below the bar. Change scores were expressed in absolute and not relative units. This means that if a performance measure fell from $80 \%$ after placebo to $70 \%$ after active drug; the change score would be $10 \%$ and not $12.5 \%$. Mean drug-placebo changes were tested for significant departure from zero by 2-tailed t-tests, for each group separately. Differences between the groups' mean changes were tested by 2-tailed t-tests for independent samples. Willingness to drive data were again analyzed for assessing differences between drug and placebo conditions using Cochran's Q-test. Differences between the respective groups' willingness to drive after drug treatments were analyzed by Mann-Whitney's U-test. Correlations were computed by Pearson's $\mathrm{r}$ and tested for significant departure from zero by 2-tailed t-tests.

Preliminary analyses were executed to determine whether change scores were significantly different between subjects who received placebo in the first, and active drug in the second 
condition and those who received treatments in reversed order. Significant differences were generally absent and are therefore mentioned only in the exceptional cases where these were significant.

\section{RESULTS}

\section{Plasma Concentrations of the Drugs}

Table 6.6 shows mean, median and range of [THC] and [THC-COOH] in the marijuana group and [EtOH] in the alcohol group. In the marijuana group, [THC] and [THC-COOH] were in the expected range. In the alcohol group, mean $[\mathrm{EtOH}]$ at $t=35$ was somewhat lower than expected. Furthermore, the fall in [EtOH] to $t=95$ was less than expected from alcohol's pharmacokinetics; a fall of $.02 \%$ per hour is commonly seen during the elimination phase in subjects who fast prior to alcohol intake. This probably means that peak [EtOH] generally occurred during the driving test and was not much higher than measurements taken before and after. This situation contrasts to that for the subjects in the marijuana group whose [THC] reached its peak before the test and fell much more rapidly during it. Average plasma concentrations were significantly different from zero in all cases $(p<.001)$.

Table 6.6 Mean, median and range of [THC] and [THC-COOH] in $\mathrm{ng} / \mathrm{ml}(\mathrm{N}=14)$, and of [EtOH] in $\mathrm{g} \%(\mathrm{~N}=16)$.

\begin{tabular}{lllllll}
\hline & \multicolumn{2}{c}{ [THC] } & \multicolumn{2}{c}{ [THC-COOH] } & \multicolumn{2}{c}{ [EtOH] } \\
& $t=20$ & $t=80$ & $t=20$ & $t=80$ & $t=35$ & $t=95$ \\
mean & 10.5 & 2.3 & 7.5 & 5.6 & .034 & .028 \\
median & 7.8 & 1.9 & 6.1 & 4.3 & .034 & .028 \\
range & $4.3-31.2$ & $1.1-4.9$ & $2.9-18.9$ & $1.7-15.1$ & $.013-.050$ & $.018-.036$ \\
\hline
\end{tabular}

\section{Perceived "high" and "drunkness"}

Mean levels of intoxication reported by both groups varied from placebo to drug conditions in a remarkable manner. Few subjects in either group reported feeling intoxicated after placebo and their average levels were about $5 \%$ of maximum personal experience. After both THC and alcohol these levels rose to about $35 \%$ when the respective groups were about to begin driving, then declined to $25 \%$ at the end of driving. Means and standard errors of differences (SED) in reported intoxication are shown in Figure 6.2.

T-tests showed that ratings of intoxication after active drugs were significantly different from zero in the marijuana and alcohol groups, both before $\left(\mathrm{T}_{13}=4.36 \& \mathrm{~T}_{15}=5.20\right.$, respectively; $p<.001)$ and after driving $\left(\mathrm{T}_{13}=3.44 \& \mathrm{~T}_{15}=5.23\right.$, respectively; $\left.p<.001\right)$.

Subjects' responses to the question of whether they thought the administered drug was active or placebo showed that they were well aware of what they smoked or drunk. In the marijuana group, $93 \%$ of the subjects correctly identified the placebo cigarette when it was administered, and $79 \%$, the active drug. In the alcohol group, $94 \%$ of the subjects correctly identified the placebo alcohol when it was administered, and $87 \%$, the active drug. 


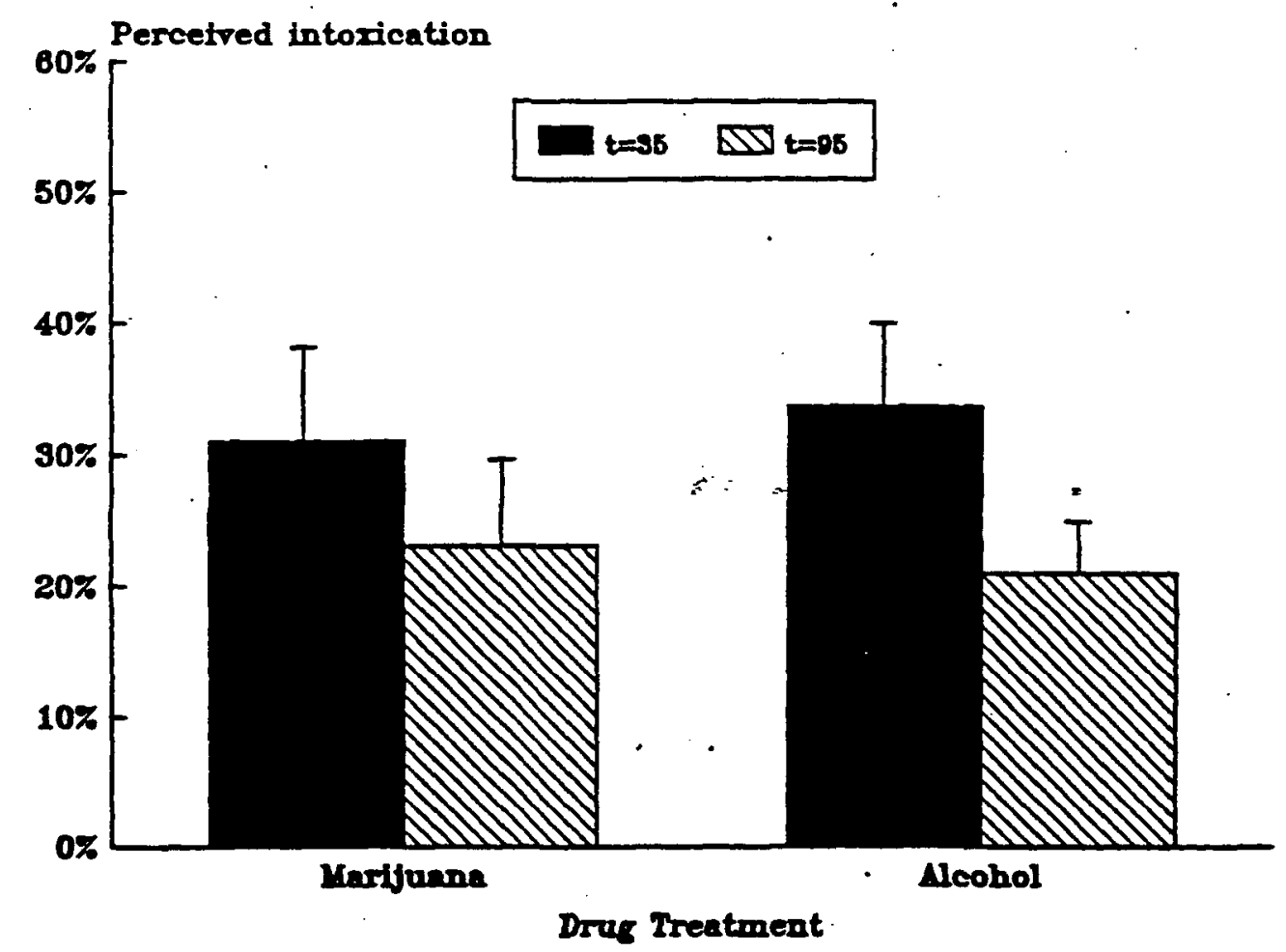

Figure 6.2 Mean (+SED) changes in perceived intoxication by Drug and Time, relative to placebo. Raw scores were expressed as percentage of maximum personal experience.

\section{Driving Performance Measured by the Molecular Approach}

Though some of the changes were positive, indicating better performance after drug and some were negative, showing the opposite, no mean change was significant (Table 6.7). Differences between the two 'groups' changes were also not significant, although one came close: the difference between improvement shown by the marijuana group and impairment shown by the alcohol group in Car Control approached significance $(p<.059)$. In general, the effects of both drugs on driving performance, as measured by the molecular approach were very small or absent.

\section{Driving Performance Measured by the Molar Approach}

Mean Total Score obtained using the molar approach varied between $82.8 \%$ and $89.5 \%$ over the conditions of the study. Groups' mean change scores on all dependent variables are given in Table 6.8. It is apparent that THC did not significantly affect Total Score or any of the component scores. On the other hand, alcohol significantly diminished the Total Score as well as two components, Handling of Vehicle and Action in Traffic. Mean Driving Time was, within two seconds, the same for both groups following placebo, namely about $41 \frac{112}{2}$ minutes. Subjects drove $46 \mathrm{~s}$ slower after THC and $42 \mathrm{~s}$ faster after alcohol, but neither change was statistically significant. 
Table 6.7 Mean ( \pm SED) changes in driving performance scores measured by the molecular approach for the marijuana $(N=14)$ and alcohol group $(N=16)$; and, the significance of each change and difference between changes.

\begin{tabular}{lccrcc}
\hline Dependent Variable & \multicolumn{2}{c}{ Marijuana Group } & \multicolumn{2}{c}{ Alcohol Group } & \multicolumn{2}{c}{ Marijuana vs Alcohol } \\
& \multicolumn{1}{c}{$\Delta$} & $p<$ & $\Delta$ & $p<$ & $p<$ \\
\hline Total Score & $1.6( \pm 1.7)$ ns & $-1.5( \pm 1.7)$ ns & ns \\
& & & & & \\
Observing & $2.4( \pm 3.1)$ ns & $-0.3( \pm 2.8)$ & ns & ns \\
Car Control & $2.7( \pm 2.1)$ ns & $-2.6( \pm 1.8)$ ns & 059 \\
Judgment & $-8.3( \pm 4.8)$ ns & $-2.2( \pm 4.0)$ ns & ns \\
Other & $-3.6( \pm 4.3)$ ns & $1.3( \pm 4.4)$ ns & ns \\
& & & & & \\
Hazard Score & $6.3( \pm 17.6)$ ns & $14.6( \pm 9.4)$ ns & ns \\
Instructor Control & $0.5( \pm 0.4)$ ns & $0.4( \pm 0.5)$ ns & ns \\
Instructions & $0.1( \pm 0.6)$ ns & $0.8( \pm 0.6)$ ns & ns \\
\hline
\end{tabular}

Table 6.8 Mean ( \pm SED) changes in driving performance scores measured by the molar approach for the marijuana $(\mathrm{N}=14)$ and alcohol group $(\mathrm{N}=16)$; and, the significance of each change and difference between changes.

\begin{tabular}{|c|c|c|c|c|}
\hline \multirow{2}{*}{ Dependent Variable } & \multicolumn{2}{|c|}{ Marijuana Group } & Alcohol Group & \multirow{2}{*}{$\begin{array}{c}\text { Marijuana us Alcohol } \\
p< \\
\end{array}$} \\
\hline & $\Delta$ & $0<$ & $p<$ & \\
\hline Total Score & $-0.7( \pm 2.7)$ & ns & $-6.8( \pm 1.8) .002$ & .065 \\
\hline Vehicle Checks & $-0.6( \pm 1.5)$ & ns & $0.5( \pm 1.3)$ ns & ns \\
\hline Handling of Vehicle & $3.7( \pm 2.8)$ & ns & $-8.4( \pm 2.2) .002$ & .002 \\
\hline Action in Traffic & $-2.7( \pm 3.1)$ & ns & $-8.4( \pm 2.3) .003$ & ns \\
\hline $\begin{array}{l}\text { Observation and Unider- } \\
\text { standing of Traffic }\end{array}$ & $1.8( \pm 8.7)$ & ns & $-6.3( \pm 7.0)$ ns & ns \\
\hline Turning & $-1.8( \pm 4.9)$ & ns & $3.1( \pm 7.5)$ ns & ns \\
\hline Driving Time & $45.6( \pm 51.8)$ & ns & $-42.0( \pm 32.4) \mathrm{ns}$ & ns \\
\hline
\end{tabular}

Differences between the groups' mean change scores were also significant or nearly so. The greater drop in Total Score caused by alcohol was almost significantly different from that caused by THC $(p<.065)$. The difference between groups' changes in Handling of Vehicle, a component score, was significant $(p<.002)$ : whereas the marijuana group's performance improved slightly, that of the alcohol group deteriorated under the influence of their respective drugs. In particular, 6 items checked under the category of Handling of Vehicle discriminated between the groups' reactions to the drugs. These all pertained to how well the driver handled the vehicle through curves. Whereas THC had little effect on this ability, alcohol seemed to affect it 
strongly. This more than any other factor was responsible for the difference between the instructor's ratings of how the two groups were affected by the respective drugs.

Perceived Driving Quality and Effort to Accomplish the Test

Both groups rated their driving performance following placebo as somewhat better than "normal". Following the active drug, ratings were about $35 \%$ lower in the marijuana group, but only $5 \%$ lower in the alcohol group (Figure 6.3). This striking mean difference was substantiated by the statistical analysis. Mean change of driving quality ratings was significantly lower in the marijuana $\left(T_{13}=-3.05 ; p<.009\right)$, but not in the alcohol group. The difference between the groups' mean changes approached significance $\left(\mathrm{T}_{28}=-1.97 ; \dot{p}<.058\right)$.

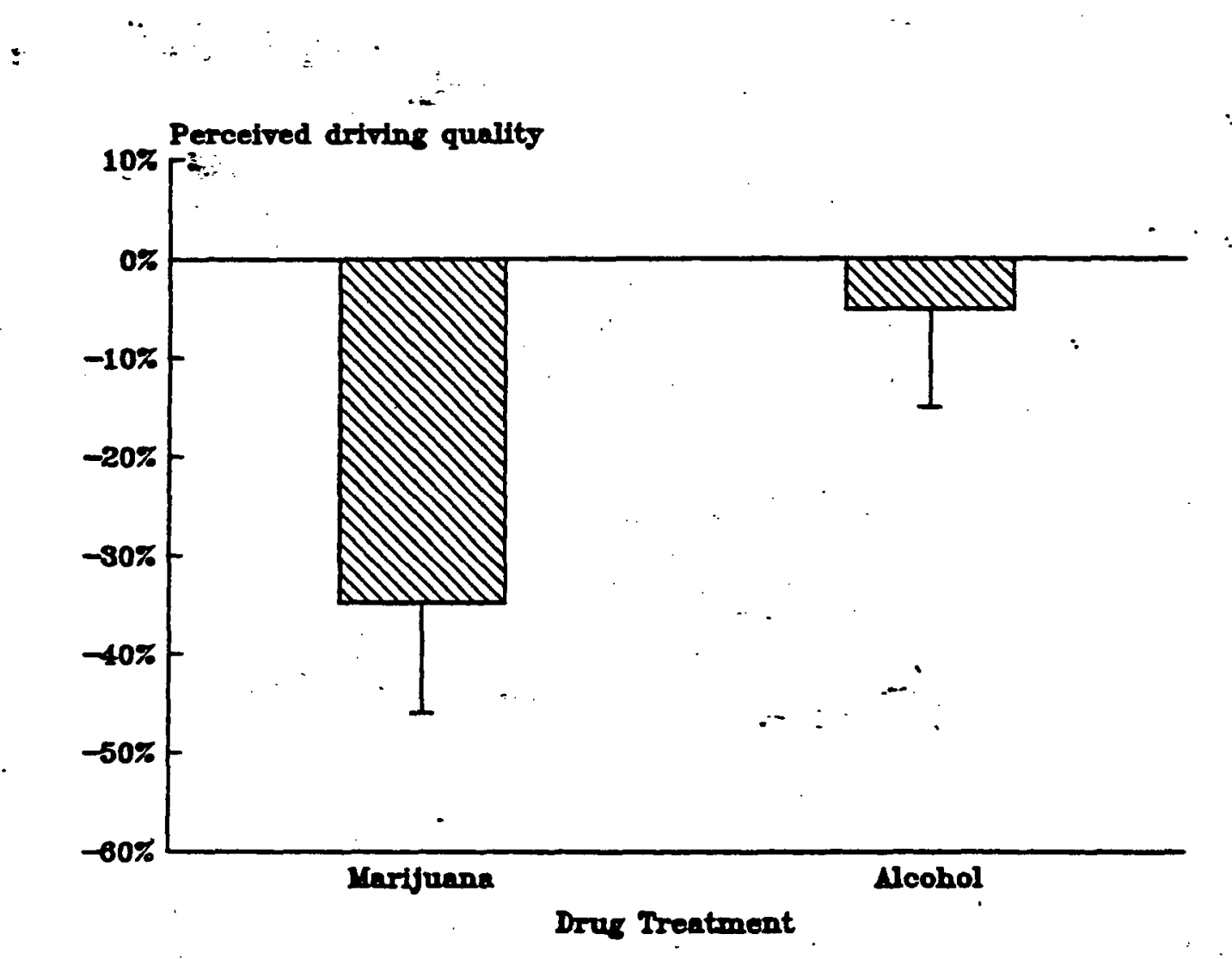

Figure 6.3 Mean (+SED) changes in perceived driving quality by Drug, relative to placebo. Raw scores were expressed as percentage of "normal".

Perceived effort to accomplish the driving test was about the same in both groups following placebo. Following the active drig; a greater increase in perceived effort was reported by the marijuana group than the alcohol group (Figure 6.4). Statistical analysis showed that increased effort ratings following the drug were only significant in the marijuana group $\left(\mathrm{T}_{13}=2.39\right.$; $p<.033)$. No significant difference between the groups' mean changes was found. 


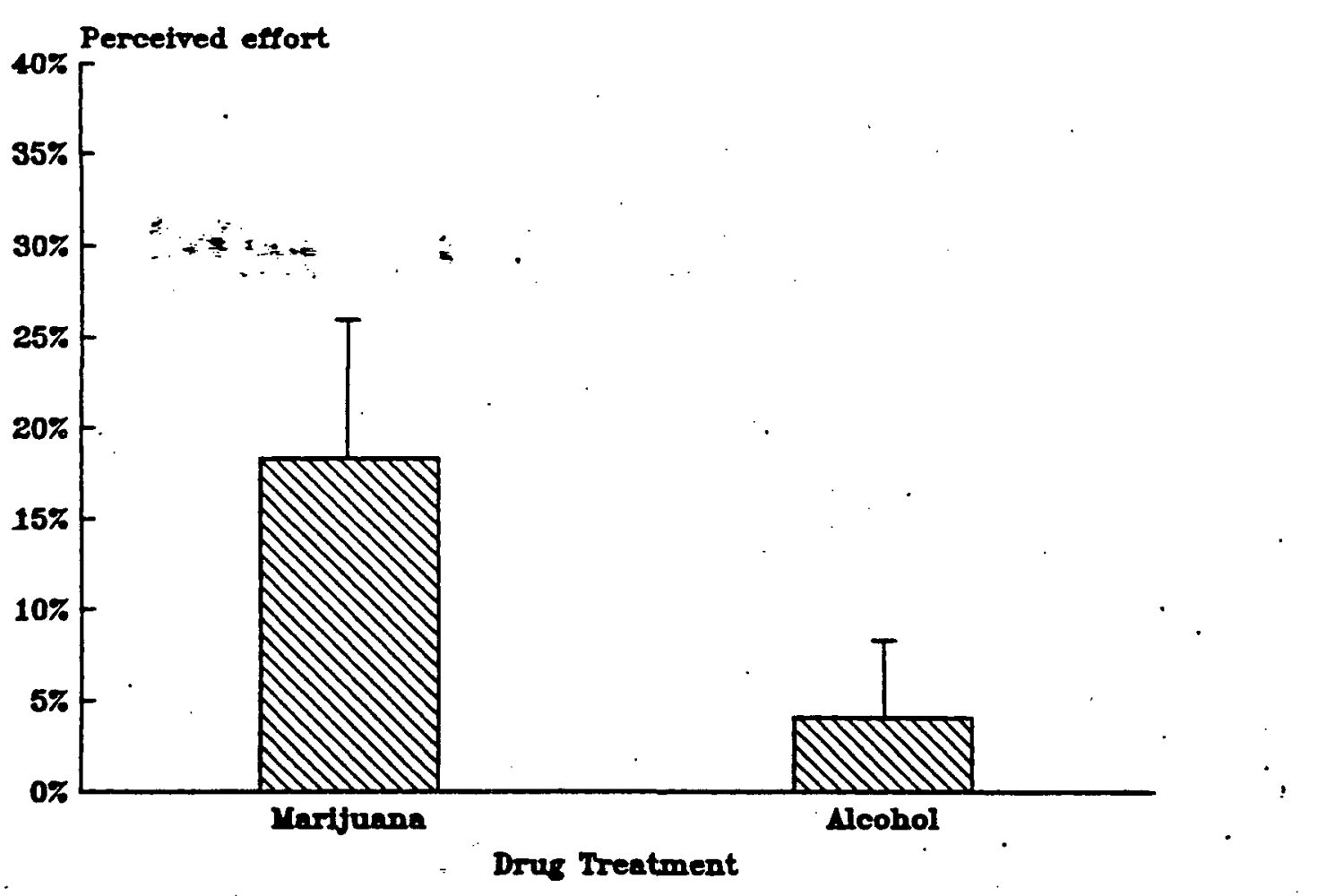

Figure 6.4 Mean (+SED) changes in perceived effort to accomplish the driving test by Drug, relative to placebo. Raw scores were expressed as percentage of maximum of scale.

\section{Willingness to Drive}

Table 6.9 presents the percentage of subjects willing to drive under specified conditions of different urgencies $(A$. unimportant though gratifying; $B$. important but avoidable; and $C$. urgent). The more urgent the reason for driving, the more subjects deciared that they would be willing to drive.

Table 6.9 Percentages of subjects willing to drive under circumstances $A, B \& C$ (see text) by treatment condition and sampling time; and, the significance of each difference between placebo and active drug condition, tested by Cochran's Q-test.

\begin{tabular}{llcccccc}
\hline & \multicolumn{3}{c}{ Alcohol Group } & \multicolumn{4}{c}{ Marijuana Group } \\
& & placebo & alcohol & $p<$ & placebo & marijuana & $p<$ \\
\hline A & $t=50$ & 100 & 38 & .006 & 86 & 50 & .044 \\
& $t=175$ & 100 & 50 & .012 & 93 & 57 & .044 \\
B & $t=50$ & 100 & 44 & .008 & 93 & 57 & .044 \\
& $t=175$ & 100 & 75 & .07 & 100 & 57 & .03 \\
C & $t=50$ & 100 & 94 & ns & 100 & 93 & ns \\
& $t=175$ & 100 & 94 & ns & 100 & 100 & ns \\
\hline
\end{tabular}


Cochran's Q-test showed that the numbers of subjects who would have driven for an unimportant reason following the active drug were significantly less than after placebo, in both groups. The same held true for the somewhat more important reason. In contrast, nearly all subjects indicated that they would have driven for an urgent reason, irrespective of the administered drug. MannWhitney's U-test failed to reveal any significant difference between the groups' percentages following the active drug.

\section{Perceived Alertness, Contentedness and Calmness}

Changes in subjective feelings of alertness, contentedness and calmness are shown in Table 6.10. Feelings of alertness were significantly diminished in both groups and at both time points following the active drug relative to placebo. Subjects felt also less content and calm following the active drugs, but the effects were smaller. Statistical tests revealed only one significant effect: alcohol producing significantly diminished feelings of contentedness measured after termination of the driving test.

Table 6.10 Mean ( $\pm \mathrm{SED}$ ) changes in subjective feelings of alertness, contentedness and calmness (raw scores being expressed as percentages of maximum) for the marijuana $(\mathrm{N}=14)$ and alcohol group $(\mathrm{N}=16)$; and, the significance of each change and difference between changes.

\begin{tabular}{|c|c|c|c|c|}
\hline Dependent Var & iable & Marijuana Group & Alcohol Group & Marijuana is Aloohol \\
\hline & & $p<$ & $p<$ & $p<$ \\
\hline Alertness & $\begin{array}{l}t=35 \\
t=95\end{array}$ & $\begin{array}{l}-15.4( \pm 4.2) .003 \\
-15.1( \pm 5.3) .014\end{array}$ & $\begin{array}{r}-18.9( \pm 3.6) .001 \\
=-20.1( \pm 3.0) .001\end{array}$ & $\begin{array}{l}\text { ns } \\
\text { ns }\end{array}$ \\
\hline Contentedness & $\begin{array}{l}t=35 \\
t=95\end{array}$ & $\begin{array}{ll}-6.2( \pm 4.0) & \mathrm{ns} \\
-5.7( \pm 3.6) & \mathrm{ns}\end{array}$ & $\begin{array}{l}-5.8( \pm 4.3) \quad \mathrm{ns} \\
-8.9( \pm 2.3) .002\end{array}$ & $\begin{array}{l}\text { ns } \\
\text { ns }\end{array}$ \\
\hline Calmness & $\begin{array}{l}t=35 \\
t=95\end{array}$ & $\begin{array}{r}-7.5( \pm 4.4) \mathrm{ns} \\
-10.0( \pm 5.2) .077\end{array}$ & $\begin{array}{ll}-1.2( \pm 4.2) & \mathrm{ns} \\
-7.3( \pm 5.3) & \mathrm{ns}\end{array}$ & $\begin{array}{l}\text { ns } \\
\text { ns }\end{array}$ \\
\hline
\end{tabular}

\section{Hand Steadiness Test}

Mean square root of total number of side contacts were about the same in the placebo conditions for both groups. The active drugs, however, affected hand steadiness differently; the number of side contacts increased after THC whereas the opposite occurred after alcohol (Figure 6.5). Statistical analysis showed that the impairment following THC was highly significant, both shortly after smoking and more than one hour later $\left(\mathrm{T}_{13}=3.96 \& 3.40 ; p<.002 \& .005\right.$, respectively), whereas the improvement following alcohol was only significant shortly after drinking $\left(\mathrm{T}_{15}=-2.29 ; p<.037\right)$. The differences between the groups' mean changes were significant both before and after the driving test $\left(\mathrm{T}_{28}=4.71 \& 3.35 ; p<.001 \& .002\right.$, respectively). 


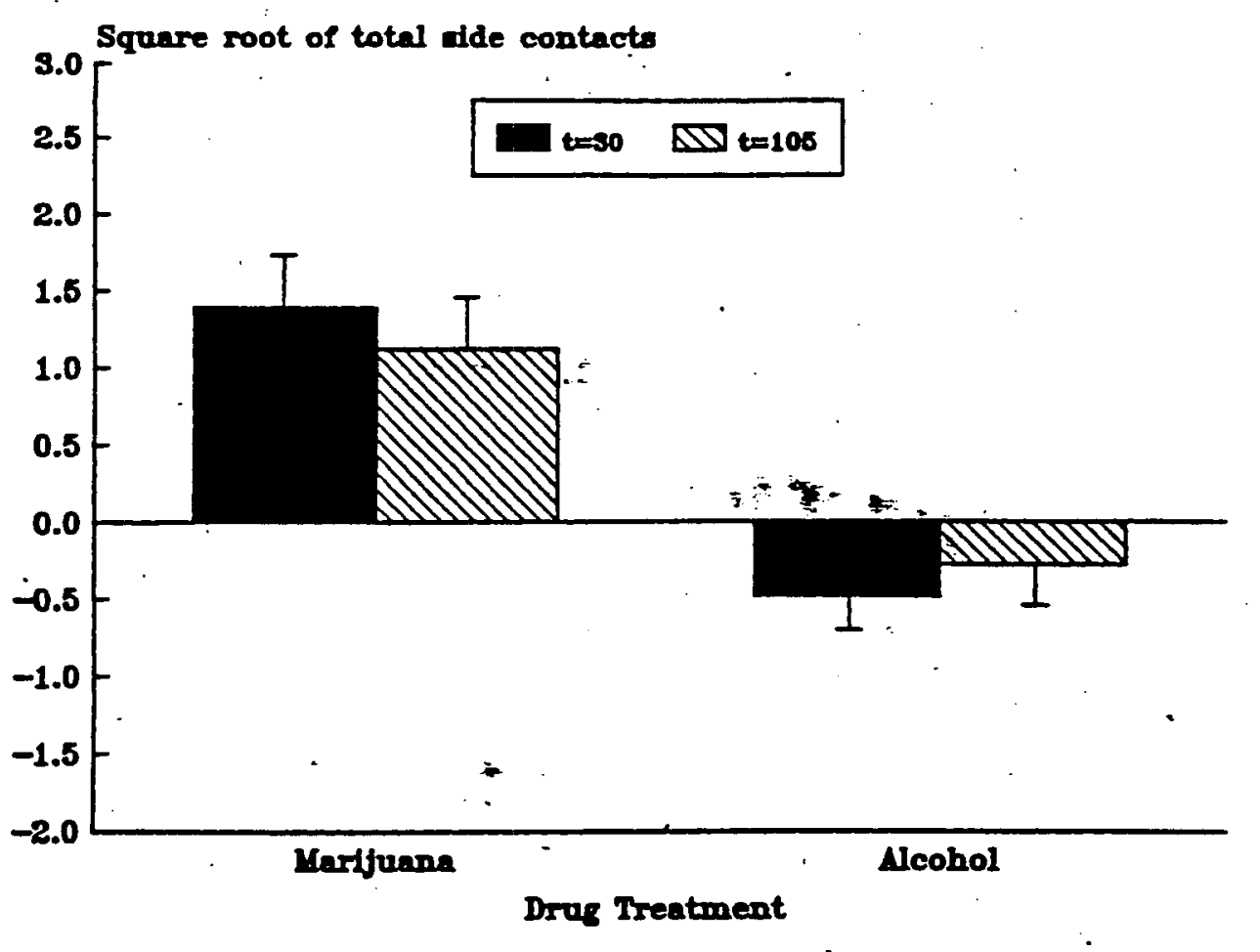

Figure 6.5 Mean (+SED) changes in the square root of total number of side contacts in the hand steadiness test by Drug, relative to placebo.

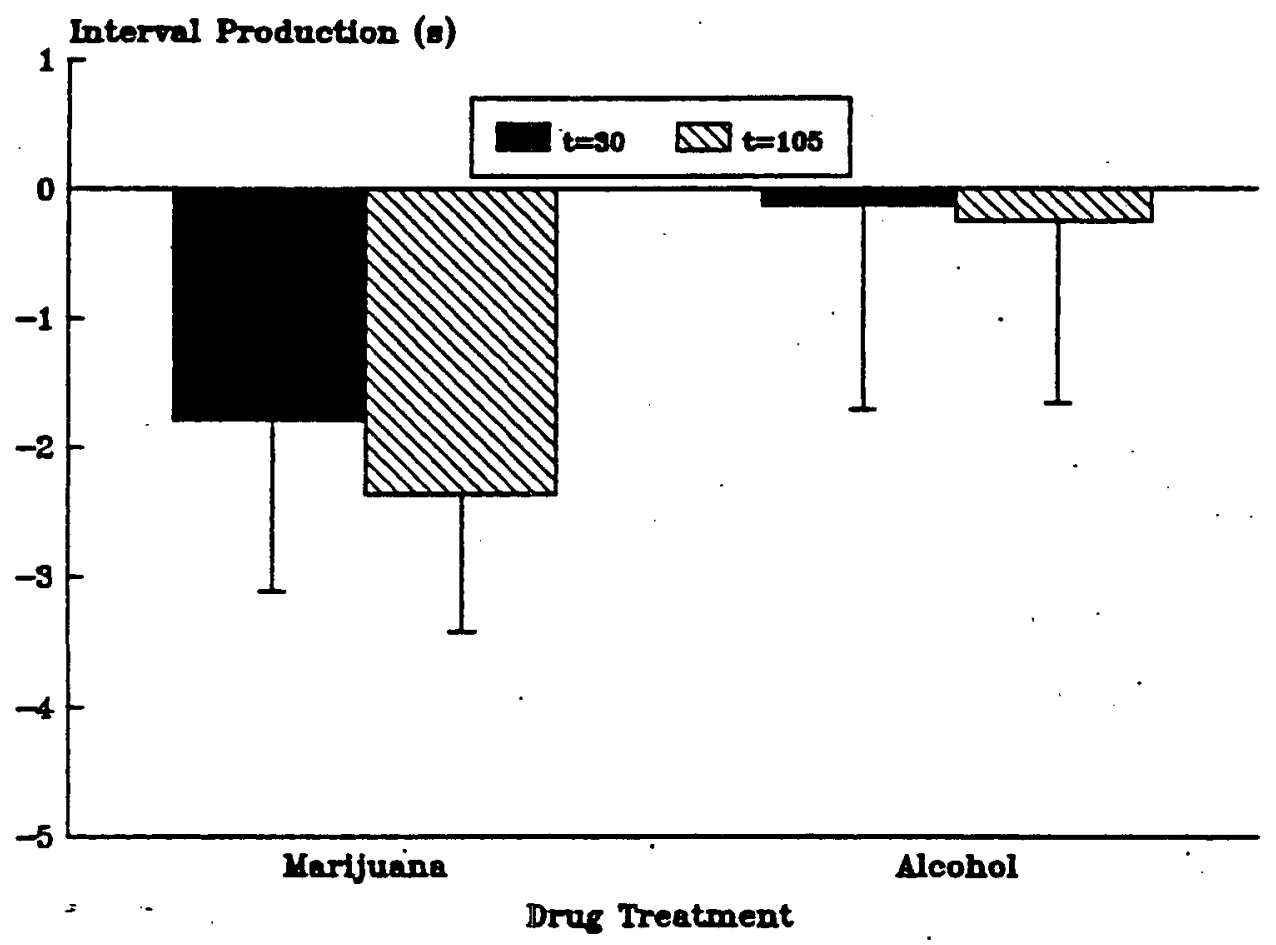

Figure 6.6 Mean (+SED) changes in attempted production of a 30-second interval by Drug, relative to placebo. 
Time Perception Test

Mean attempted production of a 30-second interval was close to the target for the alcohol group, both after placebo and active drug yielding very small, and non-significant, changes (Figure 6.6). Subjects in the marijuana group produced a foreshortened interval in both conditions, but foreshortening was greater following THC. The change in interval production was, however, only significant after, and not before, the driving test $\left(T_{13}=-2.36 ; p<.045\right)$.

\section{Inter-Subject Relations between Variables}

Relationship between Plasma Concentrations and Driving Performance. Correlational analyses were performed to determine whether driving performance was related to plasma concentrations of the drugs, measured both before and after the driving tests. The first step involved correlational analysis between raw variables; the second step, between changes in driving performance and plasma concentrations. Neither analysis yielded significant results.

Relationship between the Molecular and Molar Approach. Correlations were also computed between the raw Total Scores obtained by both the molecular and molar approach. These correlations were computed in each condition separately. For the marijuana group, the correlation between the Total Scores were $0.71(p<.005 ; 2$-tailed) and $0.54(p<.044)$ in the placebo and active drug condition, respectively. For the alcohol group, the corresponding correlations were $0.71(p<.002)$ and $0.79(p<.001)$, respectively. Differences between these correlations were not significant. The second step involved correlational analysis of change scores. This analysis revealed that changes-in Total Scores of both methods were not significantly correlated, neither in the marijuana $(r=0.05)$ nor in the alcohol group $(r=0.08)$. Relationship between Driving Performance and Driving Experience. Correlations were also computed between driving experience on one hand, and driving performance and changes in driving performance following the active drug on the other. Neither analysis revealed significant correlations. This means that driving experience of the subjects in the present study could neither predict driving performance per se nor changes following the active drug.

Relationship between Plasma Concentrations and Perceived Intoxication. Correlations of perceived "high" following THC with [THC] were $0.65(p<.013)$ shortly after smoking and $0.47(p<.093)$ following termination of the driving test. Correlations with [THC-COOH] were $0.74(p<.002)$ and $0.62(p<.018)$, respectively. Correlations of perceived intoxication following alcohol and [EtOH] were not significant $(r=0.27 \& 0.39$, respectively). Correlations of plasma concentrations with changes in perceived intoxication were nearly the same as those with the raw scores, because intoxication ratings were generally zero following placebo.

\section{DISCUSSION}

The present study showed that alcohol, administered in a dose of $0.43 \mathrm{~g}$ ethanol per $\mathrm{kg}$ of lean body mass yielding an average plasma alcohol concentration of about $0.04 \mathrm{~g} \%$, produced a significant impairment in city driving as measured by the molar approach, relative to placebo. Significant impairment was shown by changes in Total Score, and subscores describing Handling of Vehicle and Action in Traffic. Marijuana, administered in a dose of $100 \mu \mathrm{g}$ THC per $\mathrm{kg}$ of whole body weight, on the other hand, did not significantly change mean driving performance 
as measured by this approach. Neither alcohol nor marijuana significantly affected driving performance measures obtained by the molecular approach.

The first conclusion one might draw is that the methods applied for measuring driving performance did not yield exactly the same results. Yet both methods similarly measured individual differences in driving proficiency. Correlations between the total scores in all four conditions were between 0.54 and 0.79 , and as such, highly significant. Obviously both methods were able to discriminate the relatively large pre-existing differences in the subjects' respective abilities to operate the vehicle in urban traffic. Change scores were, however, not correlated; i.e. $r=0.05 \& 0.08$ in the marijuana and alcohol group, respectively. Since the molar, and not the molecular, approach was sensitive to alcohol's adverse effects, it is tempting to conclude that the latter may fail to measure some important driving deficits. That is, the molecular approach seems relatively insensitive to drug-induced changes.

The second conclusion one might draw is that the molar method, having proven its sensitivity by detecting impairment associated with a low BAC, should be sensitive to any similar impairment occurring after a correspondingly low THC dose. That it failed to show any significant change in the marijuana group's driving performance leads to the third and most important conclusion:

\section{The $100 \mu \mathrm{g} / \mathrm{kg}$ THC dose these subjects received shortly before the test did not impair their city driving performance.}

The different effects of alcohol and marijuana on driving performance can not be explained by the subjects' reported driving experience. Although subjects in the alcohol group were somewhat more experienced drivers than those in the marijuana group, the difference was not significant. Furthermore, correlational analysis showed that neither driving performance nor driving impairment was significantly related to previous driving experience. The different effects of alcohol and marijuana on driving performance can also not be explained by differences in the groups' subjective feelings. There was a remarkable correspondence between the two groups' feelings of intoxication, i.e. "high" or "drunkness". Prior to the driving test, both groups reported intoxication levels of about $35 \%$ of the highest ever experienced, and $25 \%$, after its termination. THC's significant impairing effect on hand steadiness also showed that the subjects in the marijuana group were sensitive to THC's effects.

The difference between the drugs' effects on driving may be explained, however, by compensational mechanisms, such as increased effort. Both groups reported about the same amount of effort in accomplishing the driving test following placebo. Yet only subjects in the marijuana group reported significantly higher levels of invested effort following the active drug. Previous on-road driving studies indicate that subjects may compensate for THC's adverse effects by slowing down (Hansteen et al., 1976; Casswell, 1979; Peck et al., 1986). Results of the present study were in the same direction: it took the marijuana group an average of $46 \mathrm{~s}$ longer to complete the circuit after THC than following placebo. That this difference was not significant was probably due to the real-life character of the test: unforseen obstructions and variable traffic density probably increased the error variance of this measure over what it would have been on a closed course. Thus, there was evidence that subjects in the marijuana group were not only aware of their intoxicated condition but were also attempting to compensate for it. 
There were other striking differences between the groups' reactions to their respective drugs. As stated, subjects in the marijuana group invested more effort in the test while driving under the influence of THC. The alcohol group apparently made no greater effort after the drug than placebo. Yet for all of their effort, the marijuana group rated their driving performance as being significantly worse after THC than placebo. The alcohol group who invested no special effort after the drug, not surprisingly, thought they had driven as well as following placebo. They were nonetheless driving in a deficient manner according to the instructor's ratings. These seem to

be important findings. They support both the common belief that drivers become overconfident after drinking alcohol and investigators' suspicions that they become-more cautious and selfcritical after consuming low THC doses by smoking marijuana.

These impressions were seemingly contradicted by the similarity in the groups' responses indicating their willingness to drive after the respective drugs. About $50 \%$ of both groups said they would have been unwilling to drive for less than very urgent reasons. This profession of caution seems a bit odd for the alcohol group who knew that they could not be given a dose which would produce BACs over the legal limit during the test. The "contradiction" in this case seems to be a reflection of the group's desire to give "socially desirable" answers to the questionnaire, whether the same was true for the marijuana group in the present and preceding studies is a moot point.

The laboratory performance tests also discriminated between the drugs' effects. Hand steadiness was impaired following THC and improved following alcohol, relative to placebo. The difference between the drugs' effects was significant, both before and after the driving test. Impairment after THC was about as much as that produced by the same dose in the previous study, indicating equivalent sensitivities of the present and previous groups. Production of time intervals was not affected by alcohol, but THC significantly shortened interval production, relative to placebo. This confirms the common finding that THC affects time estimation (Hollister and Gillespie, 1970; Bech et al., 1973). As usual, THC accelerated the subjects' perception of time passing. They arrived at a foreshortened production of $30 \mathrm{~s}$ and would have estimated the duration of the actual interval as longer that it actually was. Changes in driving performance were not correlated with changes in performance in either laboratory test. Nevertheless, both tests should be seriously considered for inclusion in any test battery for measuring these drugs' effects, mainly because of the different pattern of results they produced.

Plasma concentrations of THC and THC-COOH were comparable to those obtained in the previous studies. Plasma concentrations of ethanol were somewhat lower than predicted, and the fall in plasma concentration during the driving test was less than expected. This probably means that peak plasma concentrations generally occurred during the driving test. If so, it probably was about $0.04 \mathrm{~g} \%$. Ratings of "high" were significantly related to plasma concentrations of THC and THC-COOH but those of "drunkness" were not significantly related to plasma concentrations of ethanol. Drug plasma concentrations were neither related to absolute driving performance scores nor to the changes that occurred from placebo to drug conditions. With respect to THC, these results confirm the findings in previous studies. They are somewhat surprising for alcohol but may be due to the restricted range of ethanol concentrations in the plasma of different subjects.

How do the present results relate to those reported by Klonoff (1974)? He also assessed the effects of relatively low THC doses on aspects of subjects' driving performance in a city driving test using a similar molar approach. His subjects commenced driving immediately after cessation of smoking. They scored lower on judgment and concentration scales when under the influence 
of the highest, but not the lowest, dose. Behaviors that were more directly related to driving performance, e.g. lane changing, compliance with traffic signals and posted speed advisories, were not affected by the drug. Subjects in the present study commenced driving $\mathbf{3 0}$ minutes after initiation of smoking. The molar method applied in the present study did not include ratings of judgment or concentration. With the exception of these two items, neither study indicated significant effects of THC on driving performance and are, therefore, in complete agreement. 


\section{CHAPTER 7 - GENERAL DISCUSSION, CONCLUSIONS AND RECOMMENDATIONS}

\section{INTRODUCTION}

It is commonly assumed that marijuana smoking in real-life social situations delivers THC doses that seriously degrade the ability to safely operate motor vehicles; and, that the drug's users frequently drive shortly after smoking. If these premises are correct, it would follow that marijuana users are, while intoxicated, at increased risk of traffic accident involvement and constitute a safety hazard for other road users. However, the foremost impression one gains from reviewing the literature is that no clear relationship has ever been demonstrated between marijuana smoking and either seriously impaired driving performance or traffic safety. The epidemiological evidence, as limited as it is, shows that the combination of THC and alcohol is over-represented in injured and dead drivers and more so in those who actually caused the accidents. Yet there is little if any evidence to indicate that drivers who have used marijuana alone are any more likely to cause serious accidents than drug free drivers. To a large extent, the results from driving simulator and closed-course tests corroborate the epidemiological findings by indicating that THC in single inhaled doses up to about $250 \mu \mathrm{g} / \mathrm{kg}$ has relatively minor effects on driving performance, certainly less than BACs in the range $0.08-0.10 \mathrm{~g} \%$.

But how well do these findings relate to the actual driving performance of regular marijuana users? If previous experience is any guide, little of crucial importance will emerge from experimental research until it is conducted in a more realistic manner. Therefore, a series of studies were conducted to determine the dose-response relationship between THC and objectively and subjectively measured aspects of real world driving. A variety of driving tasks were employed, including maintenance of a constant speed and steady lateral position during uninterrupted highway travel, adjusting velocity to the movements of a leading car on a highway, and city driving. The purpose of applying different tests was to determine whether similar changes in performance under the influence of THC occurs in all thereby indicating a general drug effect on driving safety.

However real these tests might appear, the circumstances in which the subjects smoked the drug and drove the car were still somewhat artificial. First, the subjects consumed the drug in a neutral setting, alone or in the presence of a stranger. This was of course different from the situation wherein they normally smoke marijuana, i.e. in the company of friends who might reinforce a certain type of behavior not normally considered as conservative or prudent. Marks and Pow (1989) found that marijuana smokers derived slightly greater pleasure from THC in the company of friends than strangers from which one might infer a social amplification of the pharmacological effect. However, neither they nor any other investigators ever indicated that social amplification outlasts the situation which gives rise to it. In the absence of such evidence, we tend to discount the possibility that the unusual setting for drug administration had any effect upon subsequent driving performance. Social amplification of THC's adverse effects on driving performance might well occur if the driver were accompanied by similarly intoxicated passengers. We must therefore restrict generalizations from the studies' results to those situations 
where the intoxicated driver is doing his best to perform efficiently and is uninfluenced by friends or any other factor which might amplify the drug's adverse effects.

Secondly, subjects were aware of the fact that their driving behavior was being observed and that the accompanying driving instructor would intervene if necessary. The former is hardly avoidable in experimental studies but possibly provoked the subjects to do their utmost; the latter is a prerequisite for ensuring safety in actual driving studies and may or may not have produced nonchalance on the subjects' part resulting in poorer than normal performance. Yet according to what the subjects declared, they drove normally after placebo indicating that the experimental situation had not seriously altered their performance. Whether they drove in the test as they normally would after smoking marijuana remains open to question. The correspondence between experimental and epidemiological studies regarding THC's effects on driving performance suggests that this artificiality is also of minor concern.

The present series of studies are about the best simulation of real world driving one can reasonably achieve and have gone further toward defining the effects of marijuana smoking on actual driving performance than any of its predecessors. The results found in the successive studies were discussed at full length in the respective chapters. In this chapter, results of the separate studies will be integrated to provide answers to some important questions concerning marijuana's influence on driving performance. First however, the implications of the pilot study's major result will be discussed, i.e. the THC dose that marijuana users prefer to achieve their desired "high". In the following section, the driving tests will be discussed in terms of the kinds of mental operations they require. Some further remarks provided in that section concern the relevance of the driving tests for traffic safety. The major part of this chapter will be addressed to the most important issue, i.e. what the results indicate as the real effects of marijuana smoking on driving performance. Finally, attention will focus on the relationship between plasma drug concentrations and drug-induced driving impairments. This chapter will end with a list of conclusions and recommendations.

\section{THC DOSES}

To avoid arbitrarily selecting an unrealistic maximum THC dose for the driving studies, it was necessary to establish the dose which marijuana users actually prefer for achieving their desired "high". A pilot study met this requirement. The study showed that marijuana users prefer higher THC doses than those previously administered in experimental studies. Previous THC doses were generally between 100 and $200 \mu \mathrm{g} / \mathrm{kg}$, including the remaining butt, whereas the subjects in the pilot study preferred an average dose of $300 \mu \mathrm{g} / \mathrm{kg}$, excluding the butt. This either means that today's marijuana users prefer higher doses than in the past or that investigators failed to administer realistic doses in previous studies, or both.

Ohisson et al. (1980) compared THC's effects after three different routes of drug administration. In the smoking condition, subjects were allowed to smoke a marijuana cigarette containing $19 \mathrm{mg}$ THC in their own manner such as to obtain the maximum desired "high". Comparing the weight of the remaining butt with the unlit cigarette revealed that the eleven males participating in the study had smoked $13.0 \mathrm{mg}$ THC on the average. Ohlsson et al. failed to repor the subjects' mean weight but, assuming that it was $70 \mathrm{~kg}(154 \mathrm{lb})$ on average, this would equal to a THC dose of nearly $200 \mu \mathrm{g} / \mathrm{kg}$. Perez-Reyes et al. (1982) compared THC's effects after smoking marijuana cigarettes of three different potencies. Three males and three 
females participated in that study and the same procedures were applied to determine the amount of THC consumed as in Ohlsson's study. It appeared that the amount of THC consumed was proportional to the THC content, or potency, of the cigarette. The subjects consumed $9.7,12.8$ and $16.9 \mathrm{mg}$ THC when smoking cigarettes containing $1.32 \%, 1.97 \%$ and $2.54 \% \mathrm{THC}$, respectively. Perez-Reyes et al. also failed to report the subjects' mean weight but, for a $70 \mathrm{~kg}$ (154 lb) person, the weight calibrated amount of THC consumed would have been 140, 180 and $240 \mu \mathrm{g} / \mathrm{kg}$, respectively. These results indicate that the potency of the marijuana cigarette is a major contributor to the preferred dose. Ohlsson failed to mention the potency of the marijuana cigarettes they used which makes a comparison with the data of Perez-Reyes impossible. The potency of the eigarettes used in the present pilot study was $2.57 \%$, nearly the same as the highest in Perez-Reyes' study, and our subjects smoked on the average a THC dose of $20.8 \mathrm{mg}$, or $308 \mu \mathrm{g} / \mathrm{kg}$. This might be an indication that users presently prefer higher doses than in the early eighties. However, there is one important distinction between these earlier studies and the present one that may account for the increase. Our subjects were allowed to smoke a second, and even a third, cigarette to achieve their desired "high". Subjects in previous studies were given only one cigarette, maybe out of ignorance but probably because it was not the investigators' primary objective to determine the subjects' preferred dose. Consequently, the preferred dose of subjects who smoked one cigarette completely but not yet achieved their desired "high" was under-estimated. Thus, whether current users indeed prefer higher doses than ten years ago is hard to say. What is clear from the pilot study's results, is that higher THC doses than those usually administered in previous studies should be included in future ones.

The relation between preferred dose and the potency of the drug found by Perez-Reyes et al. (1982) raises two questions that might have implications for interpreting the present studies' results. The first question is, how do people regulate their THC consumption? Apparently they do not titrate brain THC concentrations to within a narrow range as tobacco smokers reliably do with nicotine (Jaffe, 1990). It's also doubtful that marijuana smokers integrate their THC concentrations over time to cease consumption when the cumulative effect approaches a certain threshold. In the earlier study the subjects may have simply ceased smoking after consuming the same volume of cigarettes, regardless of the delivered dose. But this could not have occurred in the pilot study for the present series since our subjects smoked ad lib. The probable explanation is that our subjects were somewhat aware of both the momentary brain concentration and its rate of increase and ceased smoking before the expected rise would shortly exceed the preferred effect. This procedure was accurate enough to limit the drug's effects to below what they had previously experienced as an unpleasant maximum. It was not accurate enough to attain homogeneity in the subjects' achievement of the preferred "high" in relation to the maximum (coefficient of variation: $25 \%$ ). In real life, marijuana users are usually more aware of the potency of the material they smoke, from prior experience, upon the advice of other users or even from its "street" price. The material presented to the subjects in.the pilot study was definitely unfamiliar and they may have suddenly become prudent smokers. Confirmation of this supposition came from the same subjects' consumption of THC in the subsequent driving study. Though treated with the same average dose they had preferred in the pilot study, their plasma drug concentrations were now higher. This difference was interpreted as the result of increased smoking efficiency due to familiarization with the potency of the material containing the drug.

If familiarization plays a major role in smoking efficiency and, consequently THC plasma concentrations, THC's adverse effects on performance may also be dependent on this factor. That being the case, the second and third driving studies' results would under-estimate THC's 
adverse effects on driving performance since new groups of subjects were recruited in both. However, the remarkable resemblance between effects of every THC dose on SDLP in the first and second driving studies indicates that familiarization is not a very important factor. Nevertheless, we recommend that subjects in future studies be familiarized with the potency of the drug before the beginning of performance testing.

The second question raised by the apparent relationship between the dug's potency and the preferred dose is, what the latter would have been if marijuana of much higher potency were smoked. This is an important question since potencies of marijuana are increasing rapidly. At present, potencies of marijuana cultivated in The Netherlands may contain $5-20 \% \mathrm{THC}$, and, in exceptional cases, $30 \%$. It is hard to imagine that subjects would consume as much of a cigarette containing a very high THC concentration as one containing a very low one. Future research determining marijuana users' preferred dose should also include marijuana with much higher potencies. It may then appear that Perez-Reyes et al.'s findings were attributable to their subjects' inability to discriminate among potencies spanning a narrow range. A broader range of cigarette potencies would probably allow regular marijuana users to discriminate well enough to alter their consumption and thereby come closer to administering the same preferred dose after every one.

On the basis of the pilot study's results, the highest dose of THC administered in subsequent driving studies was defined as $300 \mu \mathrm{g} / \mathrm{kg}$; other doses were 100 and $200 \mu \mathrm{g} / \mathrm{kg}$, and placebo. Marijuana cigarettes were prepared from batches obtained from NIDA with potencies varying from $1.75 \%$ to $3.58 \%$, the highest that were then available. If future research shows that current users of marijuana prefer much higher THC doses when they smoke very potent marijuana, the results of the current program can only serve as an indication of the minimal effects the drug may have on actual driving performance. If, on the other hand, it shows that users prefer a THC dose of about $300 \mu \mathrm{g} / \mathrm{kg}$ to reach their desired "high" irrespective of the potency of the drug, the results presented in this report are truly valid estimates of what may happen in daily life after smoking THC doses that induce a preferred "high".

\section{THE DRIVING TESTS}

Three different tests were applied in the experiments that progressively increased in the number of skills employed by the driver: road tracking, car following and city driving. The first is now a standardized test which, in more than $\mathbf{4 0}$ studies, has proven to be very sensitive to sedation produced by a variety of medicinal and social drugs, including alcohol. The car following and city driving test were developed following the recognition that parameters measured in the standard test fail to represent all abilities considered important for safe driving. This section will describe the differences between the driving tests and the relevance of each to traffic safety.

The difference between the three driving tests can be characterized in many ways. The one described here is that in terms of the type of information processing each requires. Two distinct types of human information processing can be distinguished (Shiffrin and Schneider, 1977): "automatic" and "controlled". The former is capable of accepting an enormous volume of perceptual information at a relatively high rate to mediate coordinated multi-effector responding in a normally stable input-output relationship. This process is not generally under voluntary control, proceeds in parallel with the stream of consciousness and involves no decision making. Controlled information processing is much slower but highly adaptive. It begins with the 
conscious perception of a discrete event or situation. Identification of the meaning of that perception is made by comparing it with information stored in memory. A decision involving the selection and execution of a particular series of motor responses and/or the suppression of a motor routine in progress occurs next. This process can proceed in parallel with an automatic process and the integrity of both are essential for safe driving.

Aspects of both types of information processing are present in most well practiced tasks. Most would agree that skilled performance is the integrated sum of automatic and controlled information processing and that their respective weights vary constantly with the task requirements. The more controlled information processing a task requires, the more it is experienced as demanding effort. According to this concept, lateral position control in the road tracking test depends principally upon automatic information processing. The car following test requires somewhat more controlled information processing and driving in urban traffic, even more. The concept can be illustrated by the ease of having a conversation with other occupants of a vehicle while driving. It is easy to converse with them while driving on a highway in light traffic. It becomes somewhat more difficult when driving in heavy traffic and maintaining a safe headway behind cars travelling in a platoon. Conversing while driving in urban traffic is even more difficult and, at times, even impossible for most drivers. Thus, the more controlled processing involved in a particular situation, the more effort the driving task demands and the less "spare capacity" is left for having a conversation with other occupants. This concept of effort relates to task or computational demands, and often, to the allocation of attention. There exists, however, also another concept of effort which relates to brain arousal mechanisms (Kahneman, 1973; Pribram and McGuinness, 1975) or the required psychological state (Hockey, 1986). If one perceives a discrepancy between his actual state of arousal and that required to efficiently accomplish the task, he will first attempt to resolve the difference by compensatory effort, e.g. focussing attention. Failing that, he will try to reduce task demands to what can be efficiently accomplished in the deficient arousal state. The latter can be accomplished during driving by reducing speed or assuming a greater headway to compensate for slower reaction times.

It is sensible to keep the relevance of the separate driving tests for driving and traffic safety in mind while discussing THC's effects on driving performance. It is obvious that the road tracking test measures only a few aspects of driving behavior. Yet proper road tracking is a general prerequisite for safe driving. Consequently, drugs that substantially impair a driver's fundamental road tracking ability possess a real potential for adversely affecting driving safety. Whether this means that drivers under the influence of such drugs bave also a greater probability of becoming involved in a traffic accident seems plausible but has yet to be determined. The same kind of reasoning applies to the car following test. It measures only a few additional aspects of driving behavior, but their impairment is also incompatible with safe driving. The city driving test measures most aspects of driving behavior and therefore comes closest to reality. Driving in urban traffic is so common in daily life that any drug-induced impairment found with this test should be considered as the most important, though not necessarily the earliest, sign that the drug possesses properties that adversely affect traffic safety. 


\section{EFFECTS OF THC ON DRIVING PERFORMANCE}

One of the issues addressed by the first driving study was whether it would be safe to continue using the same approach for subsequent on-road studies in traffic. The first group complied with all instructions, even after high doses of THC. Changes in mood were often reported but changes in personality were never observed. Most importantly, the subjects were always able to complete every ride without major interventions by the driving instructors and their safety was never compromised. The same occurred in the subsequent studies showing that it is possible to safely study marijuana's effects on actual driving performance in the presence of other traffic. In this respect, the drug is no different from many others studied by the same investigators and their colleagues.

The standard test measured the subjects' ability to maintain a constant speed and a steady lateral position between the lane boundaries. Standard deviation of lateral position, SDLP, increased after marijuana smoking in a dose-related manner. The lowest dose, i.e. $100 \mu \mathrm{g} / \mathrm{kg}$ THC, produced a slight elevation in mean SDLP, albeit significant in the first driving study. The intermediate dose, i.e. $200 \mu \mathrm{g} / \mathrm{kg}$ THC, increased SDLP moderately; and, the highest, i.e. $300 \mu \mathrm{g} / \mathrm{kg}$ THC, substantially. It is remarkable how well the changes in SDLP following THC in the first driving study were replicated in the second, in spite of the many differences in the ways they were designed. The replication of THC's effects on SDLP substantiates the generality of these results. Other objective measures obtained by this test were much less affected by THC. Mean speed was somewhat reduced following the higher THC doses, but the effects were relatively small $(\max .1 .1 \mathrm{~km} / \mathrm{h}$ or $0.7 \mathrm{mph})$. Standard deviations of speed and steering wheel movements were unaffected by the drug. Subjective ratings of perceived driving quality followed a similar pattern as SDLP indicating that the subjects were well aware of their diminished ability to control the vehicle after marijuana smoking.

The car following test measured the subjects' ability to follow a leading car with varying speed at a constant distance. All THC doses increased mean headway, but according to an inverse dose-response relationship. This type of relationship was unexpected and probably due to the particular design of the second driving study, i.e. the ascending dose series. It means that subjects were very cautious the first time they undertook the test under the influence of THC (i.e. after the lowest dose) and progressively less thereafter. As a consequence of this phenomenon, mean reaction time to changes in the preceding car's speed also followed an inverse dose-response relationship. Statistical adjustment for this confounding by analysis of covariance indicated that reaction times would not have increased significantly if the mean headway were constant. Coefficient of headway variation increased slightly following THC. Together, these data indicate that there is no more than a slight tendency towards impairment in car following performance after marijuana smoking. They also show that subjects try to compensate for anticipated adverse effects of the drug by increasing headway; especially when they are uncertain of what these might be. As in the standard test, subjects' ratings of driving quality corresponded to the objective changes in their performance.

The city driving study measured the subjects' ability to operate a vehicle in urban traffic. For reasons mentioned in the respective chapter the THC dose in that study was restricted to $100 \mu \mathrm{g} / \mathrm{kg}$. For comparative purposes another group of subjects was treated with a modest dose of alcohol, producing a mean BAC of about $0.04 \mathrm{~g} \%$. Results of the study showed that the modest dose of alcohol, but not THC, produced a significant impairment in driving performance, relative to placebo. Alcohol impaired driving performance but subjects did not perceive it. THC 
did not impair driving performance yet the subjects thought it had. After alcohol, there was a tendency towards faster driving and after THC, slower.

The results of these studies corroborate those of previous driving simulator and closed-course tests by indicating that THC in single inhaled doses up to $300 \mu \mathrm{g} / \mathrm{kg}$ has significant, yet not dramatic, dose-related impairing effects on driving performance. They contrast with results from many laboratory tests, reviewed by Moskowitz (1985), which show that even low doses of THC impair skills deemed important for driving, such as perception, coordination, tracking and vigilance. The present studies also demonstrated that marijuana can have greater effects in laboratory than driving tests. The last study, for example, showed a highly significant effect of THC on hand unsteadiness but not on driving in than traffic.

It is a natural question why the effects of marijuana on actual driving performance appear to be so small. As in many previous investigations, subjects attempted to compensate for anticipated adverse effects of marijuana smoking. Our subjects were aware of the impairing effects of THC as shown by lower ratings of perceived driving quality. Consequently, they invested more effort to accomplish the driving tests following THC than placebo. Furthermore, in the car following test, they drove at a greater headway after marijuana smoking; and, in both road tracking and city driving tests, they slightly reduced their driving speed. Yet despite their effort, subjects were unable to fully compensate for THC's adverse effects on lateral position variability. This is because SDLP is primarily controlled by an automatic information processing system which operates outside of conscious control. The process is relatively impervious to environmental changes, as shown by the high reliability of SDLP under repeated placebo conditions, but highly vulnerable to internal factors that retard the flow of information through the system. THC and many other drugs are among these factors. When they interfere with the process that restricts SDLP, there is little the afflicted individual can do by way of compensation to restore the situation. Car following and, to a greater extent, city driving performance depend more on controlled information processing and are therefore more accessible for compensatory mechanisms that reduce the decrements or abolish them entirely.

This still leaves the question open why performance appears to.be more affected by THC in laboratory than actual driving tests. Many researchers defend the primacy of laboratory performance tests for measuring drug effects on skills related to driving on the basis of superior experimental control. Certainly some control is always necessary to reduce the confounding influence of extraneous factors that would otherwise so increase measurement error as to totally obscure the drug's effects. However, only some extraneous factors are truly sources of measurement error and others either attenuate or amplify drug effects in real driving and must be considered as relevant to a test's predictive validity. Simply eliminating all of them, first, removes their normal mediating influence on the drug effect, and secondly, affects the subject's motivation to perform the test by making it appear "unreal". Controlling the test usually involves drastic simplification and restriction of response options. The desire in doing this is to isolate a particular driving skill and determine how it changes under the influence of drugs. However, drivers always apply numerous skills in parallel and series. Should one become deficient, they are often able to compensate in a number of ways to achieve a satisfactory level of proficiency. Thus the demonstration of some particular skill decrement in the laboratory in no way indicates that this would ultimately reduce driving safety in reality. Finally there are some skills that simply can not be measured in laboratory tests, at least not easily enough to make it a routine matter. The acquisition of any skill which depends upon automatic information processing requires practice over weeks or months. After learning to drive, subjects possess such skills in 
abundance and one can only demonstrate how they vary with drug effects in the real task or a very close approximation thereof.

Profound drug impairment constituting an obvious traffic safety hazard could as easily be demonstrated in a laboratory performance test as anywhere else. But THC is not a profoundly impairing drug. It does affect automatic information processing, even after low doses, but not to any great extent after high doses. It apparently affects controlled information processing in a variety of laboratory tests, but not to the extent which is beyond the individual's ability to control when he is motivated and permitted to do so in real driving. In short, it would appear as if over-control in laboratory performance tests has resulted in a misimpression of THC's effect, incomplete in some respects and exaggerated in others. The actual driving tests may provide a more realistic impression of the drug's effects, albeit still incomplete and perhaps

- tending to minimize them with respect to more complex driving situations that come closer to "worst case".

The degree of experimental control also varied between driving tests in this series in ways affecting the subjects' motivation. This is illustrated by a comparison between the first and second driving study. The standard road tracking test was applied in both, first in the absence and then in the presence of other traffic. It was only during the former that disturbing observations of two individuals' attentional deficits caused the driving instructor to intervene. Driving in the presence of other traffic, subjects were always able to complete the rides without intervention. Lateral position control, an automatic process, did not change as a consequence of the absence or presence of other traffic. What did change was the subjects' motivation to focus attention, a controlled process. Motivation in the second study was very probably affected by recognition of the increased risk of the untoward consequences of wandering attention. This means that the intrinsic motivation produced by the reality of the test situation is an important mediator of THC's effects on performance.

Compensatory mechanisms help the driver under the influence of marijuana to maintain an effective level of performance but with an associated cost. If drivers compensate for THC's adverse effects by diminishing driving demands (e.g. by reducing speed and/or increasing headway), this will occur without a reduction in spare capacity. But if they increase effort as well (e.g. by focusing attention), it will occur at the expense of spare capacity. Less capacity would be left for simultaneously performing another task, such as conversing with passengers, using a car telephone, or handling emergency situations. The information processing capacity these situations demand may well go beyond the driver's spare capacity with the result of impaired and perhaps dangerous driving. Results of the present program show that THC increases the mental load of driving, as shown by increased effort ratings and reduced heart rate variability, and consequently reduces spare capacity. This corroborates results from previous simulator and closed-course studies that with reasonable consistency show an adverse THC effect on subsidiary task performance (Smiley, 1986). Further research is required to determine marijuana's effects on actual driving performance when the driver is simultaneously performing another task or suddenly confronted with a situation that requires a rapid adaptive response. The latter was occasionally encountered during the city driving test, but only after a low THC dose. The city driving test should therefore be repeated with subjects consuming higher THC doses.

Hazardous driving can also occur in situations that demand very little of the driver's information processing capacity. If the driving task is very monotonous and the demand is low, wandering attention may result in negligent monitoring with disastrous results. This is in fact what happened twice during the driving study on the closed road. After the highest THC dose, 
one subject failed to shift attention from the prescribed task to an unexpected event (screwdriver on the road); another failed to anticipate a normal event (end of circuit). Though even sober experienced drivers may experience similar deficits, the fact that it happened twice after the highest THC dose, and never after a lower dose or placebo, strongly suggests that drivers under the influence of THC would be unusually susceptible to attentional deficits during prolonged and monotonous driving.

How do marijuana's effects on driving performance compare to those of alcohol? There are two sources from which one can draw to answer the question. Information can be directly obtained from studies comparing THC and alcohol effects in the same experiment; and, indirectly, from studies wherein alcohol's effects were assessed using the same methods as applied in the present THC studies. As mentioned in Chapter 1, most closed-course studies on THC also measured alcohol's effects (BACs between 0.04 and $0.10 \mathrm{~g} \%$ ). It was generally concluded that THC's effects were less than alcohol's, especially at BACs above $0.08 \mathrm{~g} \%$. The city driving study in the present program also compared the effects of modest doses of alcohol and THC. For doses administered in that study, alcohol produced the greater effects. Indirect evidence conceming the relative effects of THC and alcohol can be obtained from three studies. First, the alcohol calibration study by Louwerens et al. $(1985,1987)$ which resembled our first driving study in many respects. According to their empirical equation, THC's effects on SDLP were equal to or less than that of $\mathrm{BAC}=0.07 \mathrm{~g} \%$. More recently, studies by Riedel et al. (1989) and Ramaekers et al. (1992a) measured the effects of low doses of alcohol (BACs of 0.05 and $0.03 \mathrm{~g} \%$ respectively) on SDLP. Both groups applied the standard test in the presence of other traffic, as in our second driving study, but on another highway. Mean SDLPs were respectively about 5.0 and $2.5 \mathrm{~cm}$ higher while driving after alcohol than placebo. The former elevation is greater than that produced by the highest THC dose in our study. The latter lies between the effects of 200 and $300 \mu \mathrm{g} / \mathrm{kg}$ doses, which were 1.8 and $2.9 \mathrm{~cm}$ respectively. There was some discrepancy between alcohol's effects on SDLP in the more recent studies and those predicted by the empirical equation: the former were higher than predicted. The discrepancy appears to be related to the difference between alcohol's effects on the ascending and descending phases of its pharmacokinetic profile. Louwerens measured alcohol's effects at the time when BAC was at the ascending but Riedel and Ramaekers measured them during the descending phase. Notwithstanding methodological differences among studies, both direct and indirect evidence converge on the conclusion that THC's effects after doses up to $300 \mu \mathrm{g} / \mathrm{kg}$ never exceed alcohol's at BACs of $0.08 \mathrm{~g} \%$.

How do marijuana's effects on driving performance compare to those of drugs other than alcohol? No direct comparisons have ever been made, but many studies employing the standard road tracking test were conducted for measuring other drugs' effects on SDLP during the last decade. The results from a few will be mentioned. Diazepam (Valium) given for one week in a low therapeutic dose ( $5 \mathrm{mg}$, thrice daily) caused anxious patients to drive with a mean SDLP about $7 \mathrm{~cm}$ higher than their premedication baseline (Van Laar et al., 1992). The same drug and dose given over the same period caused healthy volunteers to drive with a mean SDLP about $6 \mathrm{~cm}$ higher than placebo (Van Veggel and O'Hanlon, 1993). Lorazepam (Ativan), another anxiolytic, given twice daily for one week in a $1.5 \mathrm{mg}$ dose to healthy volunteers Volkerts et al. , 1988) and a $2 \mathrm{mg}$ dose to patients (Vermeeren et al., 1993), produced an elevation of SDLP of about $10 \mathrm{~cm}$ in both cases. Amitriptyline (Elavif), a widely prescribed antidepressant, given in a dose of $50 \mathrm{mg}$ at night and $25 \mathrm{mg}$ in the morning caused healthy volunteers to drive with a mean SDLP about $6 \mathrm{~cm}$ higher than placebo (Robbe et al., 1989). Flurazepam (Dalmane), 
a hypnotic, was administered to insomniacs and its "hang-over" effects on SDLP were measured 10-11 hours after ingestion. A $15 \mathrm{mg}$ dose of flurazepam elevated mean SDLP by about $4 \mathrm{~cm}$; a $30 \mathrm{mg}$ dose, $7 \mathrm{~cm}$. Antihistamines also cause sedation and, consequently, impair road tracking performance. Triprolidine (Actifed) increased SDLP by $3.5 \mathrm{~cm}$ after a single $5 \mathrm{mg}$ dose (Riedel et al., 1990); and, diphenhydramine $50 \mathrm{mg}$ (Benadryl Kapseals') increased SDLP by $4.5 \mathrm{~cm}$ (Ramaekers et al., 1992b). This is not to say that all psychotropic drugs produce greater elevations of SDLP than THC. Many in the same and other experiments had less effect than THC did in our studies. These examples are merely cited to indicate that THC's effects as measured in the standard test were in no way unusual. In so far as its effects on SDLP are concerned, THC was just another moderately impairing drug.

The foregoing comparisons might be misleading. THC's effects differ qualitatively from many other drugs, especially alcohol. For example, subjects drive faster after drinking alcohol and slower after smoking marijuana (Hansteen et al., 1976; Casswell, 1979; Peck et al., 1986; Smiley et al., 1987). Moreover, the simulator study by Ellingstad et al. (1973) showed that subjects under the influence of marijuana were less likely to engage in overtaking maneuvers, whereas those under the influence of alcohol showed the opposite tendency. Very importantly, our city driving study showed that drivers who drank alcohol over-estimated their performance quality whereas those who smoked marijuana under-estimated it. Perhaps as a consequence, the former invested no special effort for accomplishing the task whereas the latter did, and successfully. This evidence strongly suggests that alcohol encourages risky driving whereas THC encourages greater caution, at least in experiments. Another way THC seems to differ qualitatively from many other drugs is that the former's users seem better able to compensate for its adverse effects while driving under the influence. Weil et al. (1968) were among the earliest authors who mentioned the possibility that marijuana users can actively suppress the drug's adverse effects. They presumed that THC's effects were confined to higher cortical functions without any general stimulatory or depressive effect on lower brain centers. According to them, the relative absence of neurological, as opposed to psychiatric, symptoms in marijuana intoxication suggests this possibility. More recently, Moskowitz (1985) concluded that the variety of impairments found after marijuana smoking could not be explained by decrements in sensory or motor functions which led him to hypothesize that some important central cognitive process is impaired by THC, without saying what it is. Identification of THC's site of action would greatly enhance our understanding of the drug's psychopharmacological effects.

Epidemiological research has shown that THC is infrequently detected in the blood of fatally injured drivers as the only drug present. In most cases alcohol is also detected. The effects of the combination of THC and alcohol on actual driving performance have never been studied in the presence of other traffic. Closed-course studies have shown that the effects of both drugs, when taken in combination, are generally additive (Attwood et al., 1981; Peck et al., 1986). This may only be so for those behaviors that are similarly affected by both drugs given separately. Closer examination of the combined use is warranted in those driving situations where both drugs produce qualitatively different effects. It may well be so that alcohol reduces drivers' insight or motivation to the point where they would no longer attempt to compensate for the THC effect. As a result, the combined effects on drivers' performance could well be greater than the sum of either drug acting separately. There is therefore a great need for further research on marijuana and actual driving research, but now extended to the combination of marijuana and alcohol. 
In summary, this program of research has shown that marijuana, when taken alone, produces a moderate degree of driving impairment which is related to the consumed THC dose. The impairment manifests itself mainly in the ability to maintain a steady lateral position on the road, but its magnitude is not exceptional in comparison with changes produced by many medicinal drugs and alcohol. Drivers under the influence of marijuana retain insight in their performance and will compensate where they can, for example, by slowing down or increasing effort. As a consequence, THC's adverse effects on driving performance appear relatively small. Still we can easily imagine situations where the influence of marijuana smoking might have an exceedingly dangerous effect; i.e., emergency situations which put high demands on the driver's information processing capacity, prolonged monotonous driving, and after THC has been taken with other drugs, especially alcohol. We therefore agree with Moskowitz' conclusion that "any situation in which safety both for self and others depends upon alertness and capability of control of manmachine interaction precludes the use of marihuana". However, the magnitude of marijuana's, relative to many other drugs', effects also justify Gieringer's (1988) conclusion that "marijuana impairment presents a real, but secondary, safety risk; and that alcohol is the leading drugrelated accident risk factor". Of the many psychotropic drugs, licit and illicit, that are available and used by people who subsequently drive, marijuana may well be among the least harmful. Campaigns to discourage the use of marijuana by drivers are certainly warranted. But concentrating a campaign on marijuana alone may not be in proportion to the safety problem it causes.

\section{DRUG PLASMA CONCENTRATIONS AND DRIVING PERFORMANCE}

One of the program's objectives was to determine whether it is possible to predict driving impairment by plasma concentrations of THC and/or its metabolite, THC-COOH, in single samples. The answer is very clear: it is not. Plasma of drivers showing substantial impairment in these studies contained both high and low THC concentrations; and, drivers with high plasma concentrations showed substantial, but also no impairment, or even some improvement. The first driving study showed that impairment in the road tracking test was nearly the same in the first and second test, executed between 40-60 and 100-120 minutes after initiation of smoking, respectively. Plasma concentrations of THC and THC-COOH, however, were not the same during the tests: both were lower during the second than the first. The same pattern was found for ratings of perceived "high". It has been said that behavioral signs of intoxication, though small, outlast physiological and subjective reactions to THC (Reeve et al., 1983; Yesavage et al., 1985). To examine this hypothesis, future research should extend actual driving performance measurements to 4, 8, 16 and 24 hours after smoking. If driving impairment still occurs after THC disappears from plasma, it could mean that previous epidemiological research has underestimated the proportion of drivers who were driving under the influence of marijuana at the times their accidents occurred.

Mean speed was the only measure of driving performance that was even moderately related to plasma concentrations of the drug. Subjects with higher THC concentrations in plasma drove slower in the standard road tracking test (correlations varying from $r=-.18$ to $r=-.72$ between conditions). This effect might have been even more pronounced if the subjects had not been instructed to drive at a particular speed, and if they had had no feedback from the speedometer. 


\section{CONCLUSIONS}

The major conclusions from the present program are summarized as follows:

- Current users of marijuana prefer THC doses of about $300 \mu \mathrm{g} / \mathrm{kg}$ to achieve their desired "high".

- It is possible to safely study the effects of marijuana on driving on highways or city streets in the presence of other traffic.

- Marijuana smoking impairs fundamental road tracking ability with the degree of impairment increasing as a function of the consumed THC dose.

- Marijuana smoking which delivers THC up to a $300 \mu \mathrm{g} / \mathrm{kg}$ dose slightly impairs the ability to maintain a constant headway while following another car.

- A low THC dose $(100 \mu \mathrm{g} / \mathrm{kg})$ does not impair driving ability in urban traffic to the same extent as a blood alcohol concentration (BAC) of $0.04 \mathrm{~g} \%$.

- Drivers under the influence of marijuana tend to over-éstimate the adverse effects of the drug on their driving quality and compensate when they can; e.g. by increasing effort to accomplish the task, increasing headway or slowing down, or a combination of these.

- Drivers under the influence of alcohol tend to under-estimate the adverse effects of the drug on their driving quality and do not invest compensatory effort.

- The maximum road tracking impairment after the highest THC dose ( $300 \mu \mathrm{g} / \mathrm{kg}$ ) was within a range of effects produced by many commonly used medicinal drugs and less than that associated with a blood alcohol concentration (BAC) of $0.08 \mathrm{~g} \%$ in previous studies employing the same test.

- It is not possible to conclude anything about a driver's impairment on the basis of his/her plasma concentrations of THC and THC-COOH determined in a single sample. 


\section{RECOMMENDATIONS FOR FUTURE RESEARCH}

The recommendations for future research are summarized as follows:

- Future studies on marijuana's effects on driving performance should include the THC dose that currents users of marijuana prefer to achieve their desired "high", i.e. $300 \mu \mathrm{g} / \mathrm{kg}$.

- Subjects in future studies should be familiarized with the potency of the material providing THC before the beginning of tests designed to measure its effects on performance.

- Future research determining marijuana users' preferred dose should involve administering THC in different marijuana preparations that encompass a wide range of potencies.

- Further research is required for determining marijuana's effects on actual driving performance in the presence of other traffic when the driver is simultaneously performing another task or suddenly confronted with a situation that requires a rapid adaptive response.

- The city driving test should be repeated with subjects consuming THC doses over $100 \mu \mathrm{g} / \mathrm{kg}$.

- Further research on marijuana and actual driving research should be extended to include combinations of marijuana and alcohol.

- Future research should extend actual driving performance measurements to 4, 8, 16 and 24 hours after smoking. 


\section{REFERENCES}

Agurell S and Leander K (1971) Stability, transfer, and absorption of cannabinoid constituents of cannabis (hashish) during smoking. Acta Pharmaceutica Suecica, 8, 391-402.

Agurell S and Hollister LE (1986) Pharmacokinetics and metabolism of $\Delta^{9}$-tetrahydrocannabinol:

Relations to effects in man. Alcohol, Drugs and Driving: Abstracts and Reviews, 2, 61-78.

Agurell S, Halldin M, Lindgren JE, Ohlsson A, Widman M, Gillespie H and Hollister L (1986)

Pharmacokinetics and metabolism of $\Delta^{1}$-tetrahydrocannabinol and other cannabinoids with emphasis on man. Pharmacological Reviews, 38, 21-43.

Attwood DA, Williams RD, Bowser JS, McBurney LJ and Frecker RC (1981). The Effects of Moderate Levels of Alcohol and Marijuana, Alone and in Combination on Closed-Course Driving Performance. Defence and civil institute of environmental medicine (81-RSU-17), Downsview, Ontario.

Bech P, Rafaelsen L and Rafaelsen OJ (1973) Cannabis and alcohol: Effects on Estimation of Time and Distance. Psychopharmacologia, 32, 373-381.

Bond A and Lader M (1974) The use of analogue scales in rating subjective feelings. British Journal of Medical Psychology, 47, 211-218.

Borkenstein RF, Crowther RF, Schumate RP, Ziel WB and Zylman R (1964) The Role of the Drinking Driver in Traffic Accidents. Bloomington, Indiana University. Department of Police Administration.

Casswell S (1979) Cannabis and Alcohol: Effects on closed course driving behaviour. IN I Johnson (Ed.) Seventh International Conference on Alcohol, Drugs and Traffic Safety. Proceedings. Melbourne 23-28 January 1977. Australian Government Publishing Service, Canberra.

Chesher GB and Starmer GA (1983) Cannabis and Human Performance Skills. Drug and Alcohol Authority Research, Grant Report Series, Sydney, Australia, NSW.

Chesher GB (1986) The effects of alcohol and marijuana in combination: A review. Alcohol, Drugs and Driving: Abstracts and Reviews, 2, 105-120.

Chiang CW and Barnett $G$ (1984) Marijuana effect and delta-9-tetrahydrocannabinol plasma level. Clinical Pharmacology Therapeutics, 36, 234-238.

Cimbura G, Warren RA, Bennett RC, Lucas DM, and Simpson HM (1980) Drugs detected in fatally injured drivers and pedestrians in the Province of Ontario. Traffic Injury Research Foundation of Canada, Ottawa.

Cimbura G, Lucas DM, Bennett RC, Warren RA and Simpson HM (1982) Incidence and toxicological aspects of drugs detected in 484 fatally injured drivers and pedestrians in Ontario. Journal of Forensic Sciences, 27, 855-867.

Cimbura G, Lucas DM, Bennett RC and Donelson AC (1990) Incidence and toxicological aspects of cannabis and ethanol detected in 1394 fatally injured drivers and pedestrians in Ontario (1982-1984) Journal of Forensic Sciences, 35, 1035-1041.

Clark LD, Hughes R and Nakashima EF (1970) Behavioral effects of marihuana: Experimental studies. Archives of General Psychiatry, 23, 193-198.

Cook E (1986) Analytical methodology for $\Delta^{9}$-tetrahydrocannabinol and its metabolites. Alcohol, Drugs and Driving: Abstracts and Reviews, 2, 79-92. 
Council on Scientific Affairs (1985) Alcohol and the driver. Journal of the American Medical Association, 255, 522-527.

Crancer AJ, Dille JM, Delay JC, Wallace JE and Haken M (1969) Comparison of the effects of marijuana and alcohol on simulated driving performance. Science, 164, 851-854.

Daldrup T, Reudenbach G and Kimm K (1987) Cannabis, alcohol and traffic. Blutalkohol, 24, 144-156. (German: Cannabis und Alkohol im Strassenverkehr).

Davis KH, McDaniel IA, Cadwell LW and Moody PL (1984) Some smoking characteristics of marijuana cigarettes. IN S Agurell, WL Dewey and RE Willette (Eds.) The Cannabinoids: Chemical, Pharmacologic and Therapeutic Aspects (pp 97-1019). Academic Press, New York.

De Gier JJ (1979) A subjective measurement of the influence of ethyl/alcohol in moderate doses on real driving performances. Blutalkohol, 16, 363-370.

De Gier JJ, 't Hart BJ, Nelemans FA and Bergman H (1981) Psychomotor performance and real driving performance of outpatients receiving diazepam. Psychopharmacology, 73, 340-347.

Donelson AC, Cimbura G, Bennett RC and Lucas DM (1985) The Ontario monitoring project: Cannabis and alcohol use among drivers and pedestrians fatally injured in motor vehicle accidents from March 1982 through July 1984. Traffic Injury Research Foundation of Canada, Ottawa.

Dott AB (1972) Effect of Marijuana on Risk Acceptance in a Simulated Passing Task. Report ICRL-RR-71-3 DHEW Publication No. HSM-72-10010, US Public Health Service, Washington, DC.

Durnin JVGA and Womersley J (1974) Body fat assessed from total body density and its estimation from skinfold thickness: Measurements on 481 men and women aged from 16 to 72 years. British Joumal of Nutrition, 32, 77-97.

Ellingstad VS, McFarling LH and Struckman DL (1973) Alcohol, marijuana and risk taking. Vermillion Human Factors Laboratory, South Dakota University.

Ferrara SD and Rozza M (1985) Alcohol, drugs and road accidents: Epidemiological study in North-East Italy. IN S Kaye and G Meier (Eds.) Alcohol, Drugs and Traffic Safety. Proceedings of the 9th International Conference on Alcohol, Drugs and Traffic Safety, November 1983, San Juan, Puerto Rico (pp 469-485). Report No. DOT-HS-806-814, United States Department of Transportation, Washington, DC.

Fraisse P (1963) The Psychology of Time. Harper and Row, New York.

Gieringer DH (1988) Marijuana, driving, and accident safety. Journal of Psychoactive Drugs, 20, 93-101.

Hansteen RW, Miller RD, Lonero L, Reid LD and Jones B (1976) Effects of cannabis and alcohol on automobile driving and psychomotor tracking. Annals of the New York Academy of Sciences, 282, 240-256.

Hockey GRJ (1986) A state control theory of adaptation of stress and individual differences in stress management. IN GRJ Hockey, AWK Gaillard and MGH Coles (Eds.) Energetics and Human Information Processing (pp 285-298). Martinus Nijhoff Publishers, Dordrecht.

Hollister LE and Gillespie HK (1970) Marihuana, ethanol, and dextroamphetamine. Archives of General Psychiatry, 23, 199-207.

Jaffe JH (1990) Chapter 22: Drug addiction and drug abuse. IN A Goodman Gilman, ThW Rall, AS Nies and P Taylor (Eds.) Goodman and Gilman's The Pharmacological Basis of Therapeutics (pp 284-324). Pergamon Press, New York. 
Jessor R, Donovan JE and Costa F (1986) Psychosocial correlates of marijuana use in adolescence and young adulthood: The past as prologue. Alcohol, Drugs and Driving: Abstracts and Reviews, 2, 31-50.

Jex HR, McDonnell JD and Phatak AV (1966) A "critical" tracking task for manual control research. Institute of Electrical and Electronic Engineers Transactions on Human Factors in . Electronics, 7, 138-145.

Johnston LD, O'Malley PM and Bachman JG (1992) Smoking, Drinking, and Illicit Drug Use among American Secondary School Students, College Students, and Young Adults, 19751991. Institute for Social Research, University of Michigan; USA.

Jones MH (1978) Driver Performance Measures for the Safe Performance Curriculum. Traffic Safety Center, Institute of Safety and Systems Management, University of South California, Los Angeles, CA (DOT HS 803 461).

Kahneman D (1973) Attention and Effort. Prentice-Hall, New Jersey.

Kapteyn TS, Bles W, Njiokiktjein Ch, Rodde L, Massen CH and Mol JMF (1983) Standardization in platform stabilometry being part of posturography. Agressologie, 24, 321-326.

Klonoff H (1974) Marijuana and driving in real-life situations. Science, 186, 317-323.

Lindgren JE, Ohlsson A, Agurell S, Hollister L and Gillespie H (1981) Clinical effects and plasma levels of delta-nine-tetrahydrocannabinol in heavy and light users of cannabis. Psychopharmacology, 74, 208-212.

Louwerens JW, Gloerich ABM, De Vries G, Brookhuis KA and O'Hanlon JF (1985) De Invloed van Verschillende Bloedalcoholspiegels op Objectief Meetbare Aspekten van Feitelijk Rijgedrag. Technical Report No. VK 85-03, Traffic Research Centre, University of Groningen.

Louwerens JW, Gloerich ABM, De Vries G, Brookhuis KA and O'Hanlon JF (1987) The relationship between drivers' blood alcohol concentration (BAC) and actual driving performance during high speed travel. IN PC Noordzij and R Roszbach (Eds.) Alcohol, Drugs and Traffic Safety. Proceedings of the 10th International Conference on Alcohol, Drugs and Traffic Safety, September 9-12, 1986, Amsterdam, The Netherlands (pp 183-192). Excerpta Medica, Amsterdam.

Marks. DF and Pow GM (1989) Cannabis and human social behaviour. Human Psychopharmacology, 4, 283-290.

Mason AP and McBay AJ (1984) Ethanol, Marijuana, and other drug use in 600 drivers killed in single-vehicle crashes in North Carolina, 1978-1981. Joumal of Forensic Sciences, 29, 987-1026.

Maykut MO (1985) Health consequences of acute and chronic marihuana use. Progress in Neuro-Psychopharmacology and Biological Psychiatry, 9, 209-238.

Mendelson JH (1987) Marijuana. IN HY Meltzer (Ed.) Psychopharmacology. The Third Generation of Progress (pp 1565-1571). Raven Press, New York.

Meyman TF and Zijlstra FRH (1986). The measurement of perceived effort. IN DJ Oborne (Ed.) Contemporary Ergonomics (pp 242-247). Taylor and Francis, London.

Milstein SL, MacCannell K, Karr G and .Clark S (1975) Marijuana-produced impairments in coordination. Journal of Nervous and Mental Disease, 161, 26-31.

Möller MR, Dörr G and Warth S (1992) Simultaneous quantitation of delta-9-tetrahydrocannabinol (THC) and 11-nor-9-carboxy-delta-9-tetrahydrocannabinol (THC-COOH) in serum by GC/MS using deuterated internal standards and its application to a smoking study and forensic cases. Journal of Forensic Sciences, 37, 969-983. 
Moskowitz H (1985) Marijuana and driving. Accident Analysis and Prevention, 17, 323-346. Moskowitz H, Hulbert S and McGlothlin W (1976) Marijuana: Effects on simulated driving performance. Accident Analysis and Prevention, 8, 45-50.

Moskowitz H, Sharma S and Ziedman K (1981) Duration of skills performance impairment. Proceedings of the 25th Conference of the American Association for Automotive Medicine, 87-96.

Mulder G (1980) The Heart of Mental Effort: Studies in the Cardiovascular Psychophysiology of Mental Work: Doctoral Dissertation, University of Groningen, Groningen, The Netherlands.

Mulder G and Mulder LNM (1981) Information processing and cardiovascular control. Psychophysiology, 14, 392-402.

Murray JB (1985) Marijuana's effects on human cognitive functions, psychomotor functions, and personality. The Journal of General Psychology, 113, 23-55.

O'Hanlon JF, Haak TW, Blaauw GJ and Riemersma JBJ (1982) Diazepam impairs lateral position control in highway driving. Science, 217, 79-81.

O'Hanlon JF, Brookhuis KA, Louwerens JW and Volkerts ER (1986) Performance testing as part of drug registration. IN JF O'Hanlon and JJ de Gier (Eds.) Drugs and Driving (pp 311330). Taylor and Francis, London.

Ohlsson A, Lindgren JE, Wahlén A, Agurell S, Hollister LE and Gillespie HK (1980) Plasma delta-nine-tetrahydrocannabinol concentrations and clinical effects after oral and intravenous administration and smoking. Clinical Pharmacology and Therapeutics, 28, 409-416.

Overall JE and Rhoades HM (1987) Adjusting p values for multiple tests of significance. IN HY Meltzer (Ed.) Psychopharmacology. The Third Generation of Progress (pp 1013-1018). Raven Press, New York.

Owens SM (1981) Marijuana Use among Drivers in Fatal Single-Vehicle Accidents. Ph.D. dissertation, University of North Carolina, Chapel Hill, NC.

Peck RC, Biasotti A, Boland PN, Mallory C and Reeve V (1986) The effects of marijuana and alcohol on actual driving performance. Alcohol, Drugs and Driving: Abstracts and Reviews, 2, 135-154.

Perez-Reyes M, Di Guiseppie S, Davis KH, Schindler VH and Cook CE (1982) Comparison of effects of marijuana cigarettes of three different potencies. Clinical Pharmacology and Therapeutics, 31, 617-624.

Plomp HN, Kuipers H and Van Oers ML (1990) Roken, Alcohol- en Drugsgebruik onder Scholieren vanaf 10 jaar. VU Uitgeverij, Amsterdam.

Pribram KH and McGuinness D (1975) Arousal, activation and effort in the control of attention. Psychological Review, 82, 116-146.

Rafaelsen OJ, Bech P, Christiansen J, Christup H, Nyboe J and Rafaelsen L (1973) Cannabis and alcohol: Effects on simulated car driving. Science, 179, 920-923.

Ramaekers JG, Uiterwijk MMC and O'Hanlon JF (1992a) Effects of loratadine and cetirizine on actual driving and psychometric test performance, and EEG during driving. European Journal of Clinical Pharmacology, 42, 363-369.

Ramaekers JG, Van Boxtel MPJ and O'Hanlon JF (1992b) A Study to Compare Acrivastine's and Terfenadine's Effects on Several Aspects of Driving Performance as a Function of Dose and Time after Dosing. Technical Report No. IGVG 92-25, Institute for Drugs, Safety and Behavior, University of Limburg, Maastricht, The Netherlands.

Randall RC (1990) Cancer Treatment and Marijuana Therapy. Galen Press, Washington, DC. 
Reeve VC, Robertson WB, Grant J, Soares JR, Zimmerman EG, Gillespie HK and Hollister LE (1983) Hemolyzed blood and serum levels of $\Delta^{9}$-THC: Effects on the performance of roadside sobriety tests. Journal of Forensic Sciences, 28, 963-971.

Relman AS (1982) Marihuana and health. New England Joumal of Medicine, 306, 603-604.

Riedel WJ, Schoenmakers EAMM and O'Hanlon JF (1989) Sedation and performance impairment with antihistamines. IN Makaliner (Ed.) Management of Allergy in the 1990's. Hans Huber, Toronto.

Riedel WJ, Ramaekers JG, Uiterwijk MMC and O'Hanlon JF (1990) Higher doses of terfenadine and loratadine; acute and subchronic effects on psychomotor and actual driving performance. Technical Report No. IGVG 90-08, Institute for Drugs, Safety and Behavior, University of Limburg, Maastricht, The Netherlands.

Robbe HWJ, Schoenmakers EAJM and O'Hanlon JF (1989) Paroxetine and Amitriptyline: Acute and Subchronic Effects on Psychomotor and Actual Driving Performance. Technical Report No. IGVG 89-04, Institute for Drugs, Safety and Behavior, University of Limburg, Maastricht, The Netherlands.

Sharma S and Moskowitz H (1975) Marihuana effects on a critical tracking task. Proceedings of the 19th Conference of the American Association for Automotive Medicine, 285-291.

Shiffin RM and Schneider W (1977) Controlled and automatic human information processing: II. Perceptual learning, automatic attending, and a general theory. Psychological Review, 84, 127-190.

Simpson HM (1986) Epidemiology of road accidents involving marijuana. Alcohol, Drugs. and Driving: Abstracts and Reviews; 2, 15-30.

Smiley AM, Moskowitz $\mathrm{H}$ and Ziedman K (1981) Driving simulator studies of marijuana alone and in combination with alcohol. Proceedings of the 25th Conference of the American Association for Automotive Medicine, 107-116.

Smiley AM (1986) Marijuana: On-road and driving simulator studies. Alcohol, Drugs and Driving: Abstracts and Reviews, 2, 121-134.

Smiley AM, Noy YI and Tostowaryk W (1987) The effects of marijuana alone and in combination with alcohol on driving performance. IN PC Noordzij and R Roszbach (Eds.) Alcohol, Drugs and Traffic Safety. Proceedings of the 10th International Conference on Alcohol, Drugs and Traffic Safety, September 9-12, 1986, Amsterdam, The Netherlands (pp 203-206). Excerpta Medica, Amsterdam.

Smith G, Wolynetz M, Davidson M and Poulton H (1975) Estimated blood alcohol concentrations of nighttime Canadian drivers. Traffic Injury Research Foundation (TIRF) of Canada, Ottawa.

Soderstrom CA, Trifillis AL, Shankar BS, Clark WE and Cowley RA (1988) Marijuana and alcohol use among 1023 trauma patients. Archives of Surgery, 123, 733-737.

Stein AC, Allen RW, Cook ML and Karl RL (1983) A Simulator Stucty of the Combined Effects of Alcohol and Marijuana on Driving Behavior. Report No. DOT HS 806 405, System Technology, Inc., Hawthorne, CA.

Terhune KW (1982) The Role of Alcohol, Marijuana and Other Drugs in the Accidents of Injured Drivers. Technical Report to US Department of Transportation, Calspan Field Services, Inc.

Terhune KW (1986) Problems and methods in studying drug crash effects. Alcohol, Drugs and Driving: Abstracts and Reviews, 2, 1-14. 
Van der Wal HJ (1985) Roken, Drinken, Cannabisgebruik. Stichting voor Wetenschappelijk Onderzoek van Alcohol- en Druggebruik, Utrecht, The Netherlands.

Van Veggel LMA and O'Hanlon JF (1993) Effects of Ondansetron and Diazepam on Driving and Psychometric Performance in Healthy Volunteers. Technical Report No. IGVG 93-26, Institute for Drugs, Safety and Behavior, University of Limburg, Maastricht, The Netherlands.

Van Laar MW, Volkerts ER and Willigenburg APP (1992) Therapeutic effects and effects on actual driving performance of chronically administered buspirone and diazepam in anxious outpatients. Journal of Clinical Psychopharmacology, 12, 86-95.

Vermeeren A, Swijgman HF and O'Hanlon JF (1993) Acute and Subchronic Effects of Alpidem, Lorazepam and Placebo on Anxiety, Psychometric Test Performance and Actual Driving in Anxious Patients. Technical Report No. IGVG 93-19, Institute for Drugs, Safety and Behavior, University of Limburg, Maastricht, The Netherlands.

Vinciguerra V, Moore TMSW and Brennan E (1988) Inhalation marijuana as an antiemetic for cancer chemotherapy. New York State Journal of Medicine, 88, 525-527.

Volkerts ER, Abbink F, Van Laar MW, Plomp PR and Maes RAA (1988) Comparison of the Effects of Ritanserin, $5 \mathrm{mg}$, b.i.d. and Lorazepam $1.5 \mathrm{mg}$, b.i.d., upon Driving Performance in an Over-the-Road Driving Test. Technical Report No. 11-88, Netherlands Institute for Drugs and Doping Research, University of Utrecht, Utrecht, The Netherlands.

Wall ME, Sadler BM, Brine D, Taylor H and Perez-Reyes M (1983) Metabolism, disposition and kinetics of delta-9-tetrahydrocannabinol in men and women. Clinical Pharmacology and Therapeutics, 34, 352-363.

Warren RA, Simpson HM, Hilchie J, Cimbura G, Lucas D and Bennett R (1981) Drugs detected in fatally injured drivers in the Province of Ontario. IN L Goldberg (Ed.) Alcohol, Drugs and Traffic Safety. Proceedings, 8th International Conference on Alcohol, Drugs and Traffic Safety, June 15-19, 1980,. Stockholm, Sweden (pp 203-217). Almqvist and Wiksell International, Stockholm.

Weil AT, Zinberg NE and Nelson JM (1968) Clinical and psychological effects of marijuana in man. Science, 162, 1234-1242.

Williams AF, Peat MA, Crouch DJ, Wells JK and Finkle BS (1985) Drugs in fatally injured young male drivers. Public Health Reports, 100, 19-25.

Yesavage JA, Leirer VO, Denari M and Hollister LE (1985) Carry-over effects of marijuana intoxication on aircraft pilot performance: A preliminary report. American Journal of Psychiatry, 142, 1325-1329.

Zijlstra FRH and Van Doorn L (1985) The Construction of a Scale to Measure Perceived Effort. Technical Report, University of Delft, Delft, The Netherlands.

Zinberg NE (1979) On cannabis and health. Journal of Psychedelic Drugs, 11, 135-144. 
:

$1,14:$

1

APPENDIX A

Questionnaires

.. - 
Subjective Intoxication and Willingness to Drive Questionnaires

Please rate your feeling of "high" (or "drunkness" after drinking alcohol) as a percentage of the maximum ever experienced.

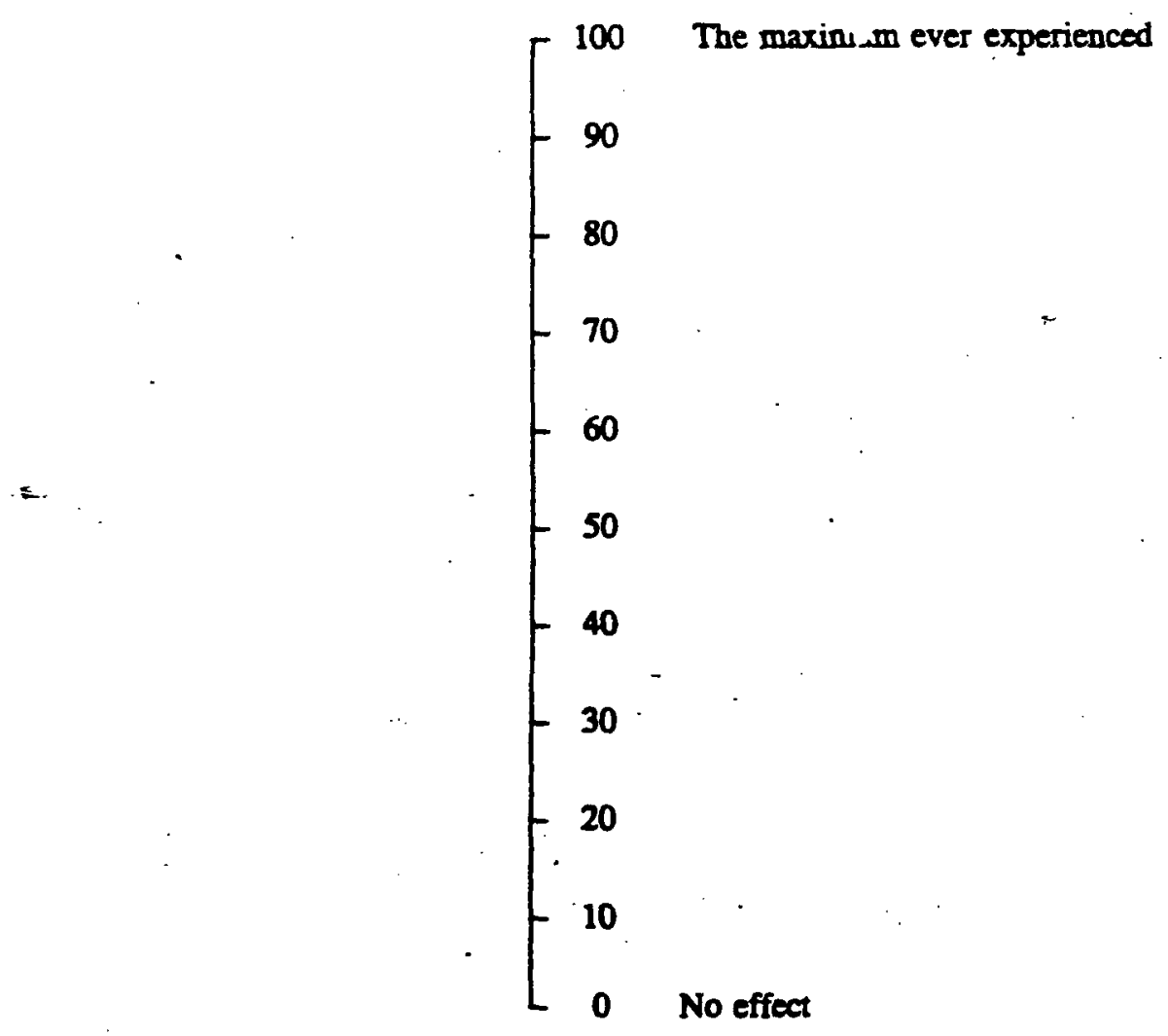

Would you artempt to drive for a set distance if the reasons were:

A. unimportant though gratifying, such as for transporting a friend to another-party $\quad$ Y / N

B. important but avoidable, such as for transporting a mildly sick friend home when he would otherwise have to call a taxi

C. urgent, such as transporting a severely sick infant to the hospital 
Mood Rating Scale (Bond and Lader, 1974)

This form is a mood rating scale and the intention is to measure your feelings as they are at this moment. The instructions are given below. Please read them carefully and proceed. Have you any questions?

1. Please rate the way you feel in terms of the dimensions given below:

2. Regard the line as presenting the full range of each dimension.

3. Rate your feelings as they are at the moment.

4. Mark clearly and perpendicularly across each line.

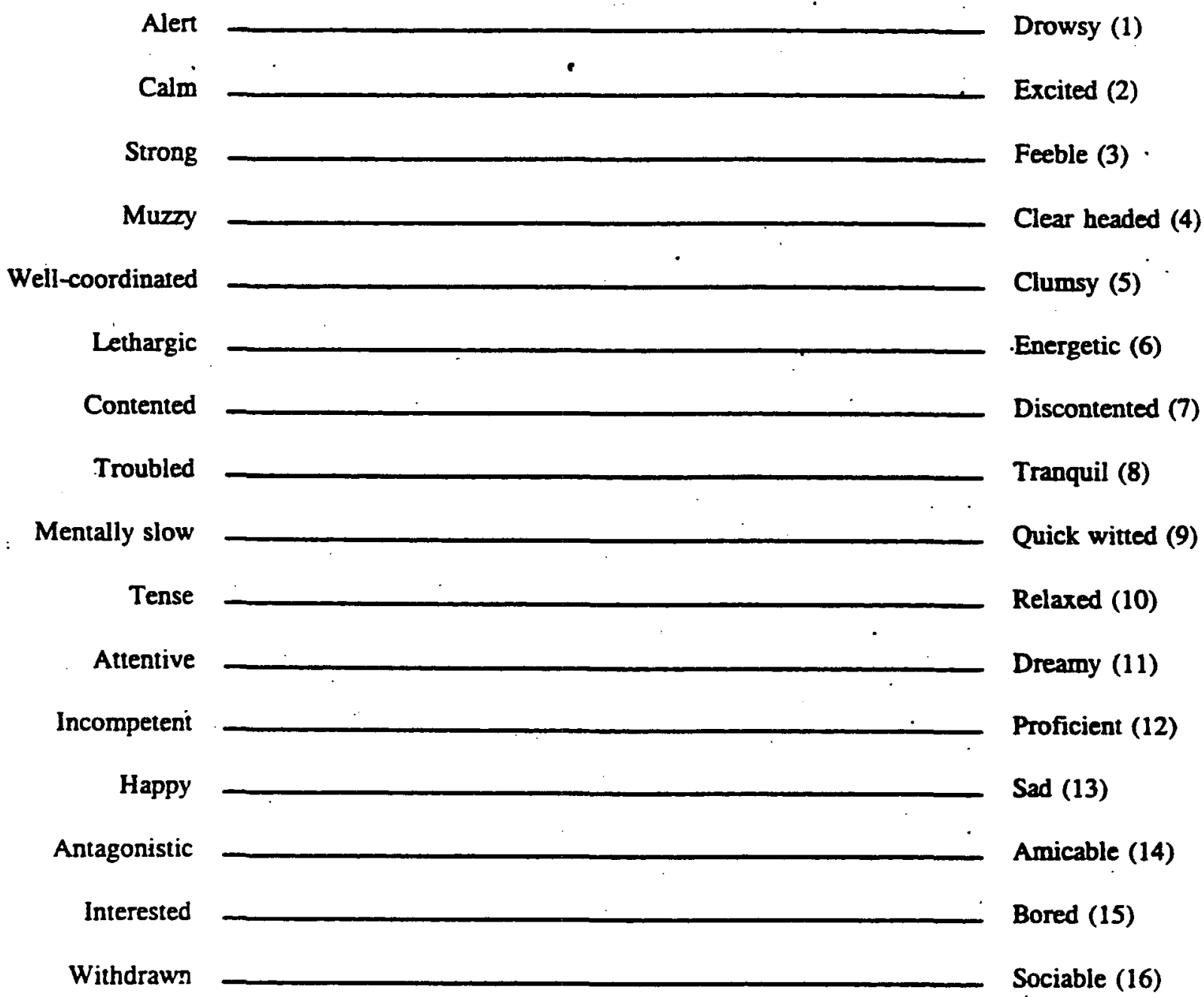

Do you think you received placebo or active drug ? Placebo / Active

Scores on these scales were grouped to form 3 cluster scores for measuring the corresponding factors: alerness $(1,3,4,5,6,9,11,12,15)$, contentedness $(7,8,13,14,16)$ and calmness $(2,10)$. 
Perceived Effort Scale (Zijlstra and Van Doorn, 1985)

Please rate the effor made while performing the driving test.

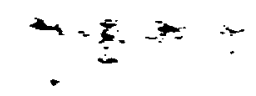

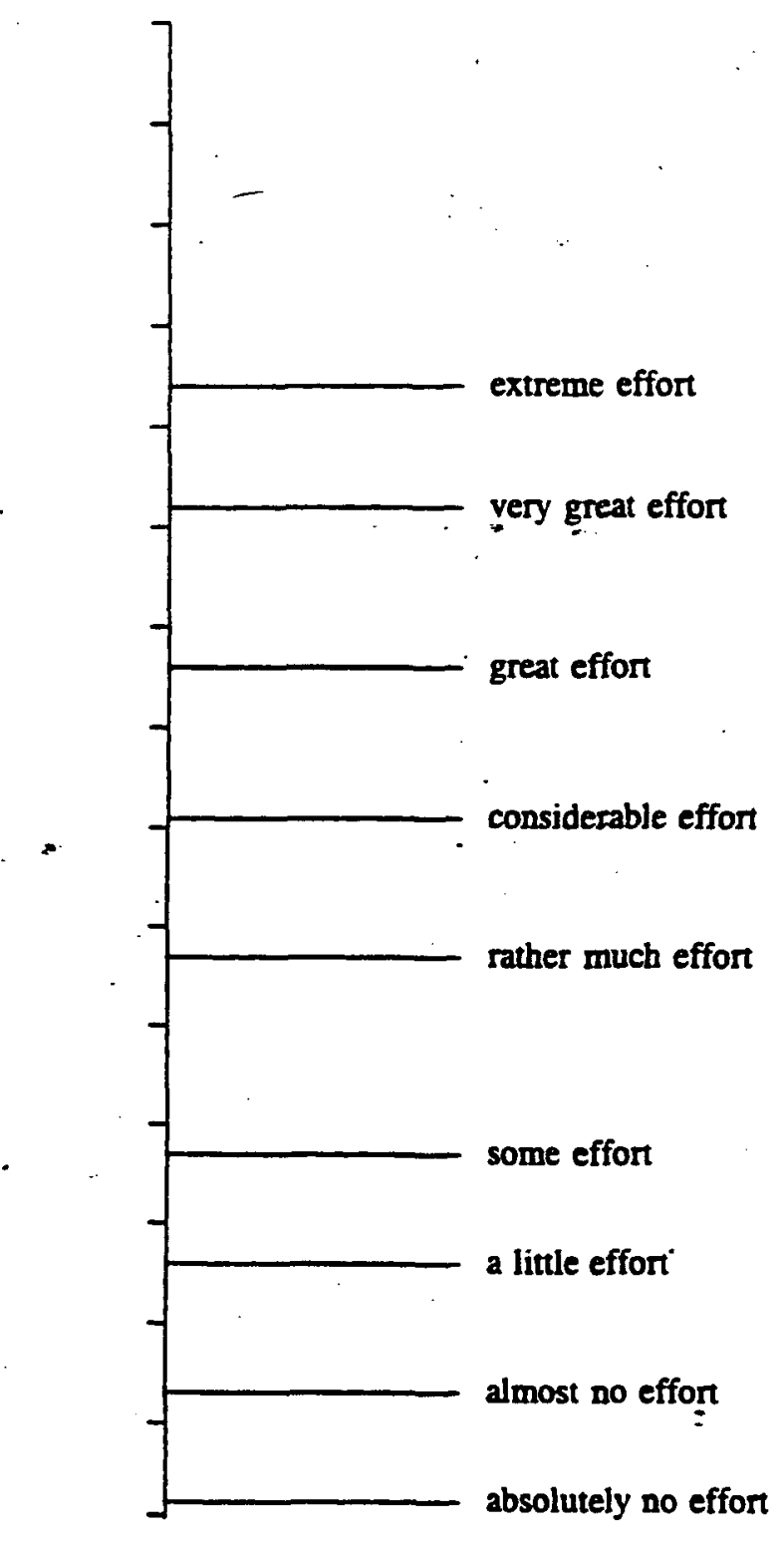


Perceived Driving Quality Scale (translated from Dutch)

Please rate the quality of your driving in the test you just finished.

I drove exceptionally well

\section{I drove normally}

I drove exceptionally poorly

$-122-$ 
APPENDIX B

Driving Proficiency Tests

$\bullet$ 


\section{Left Turn}

Path (P)

High, medium or low traffic density.

Correct Response

Left Turn

Speed (S)

High and Medium

Density Traffic

Correct Response
Lefi Turn

Speed (S)

Low Density Traffic

Correct Response
The driver will follow a standard path. A standard path runs from the center of the left-most lane of the original street to the center of the left-most lane of the intersecting street". The turn must commence at a point one-third to one-half of the way into the intersection. The path passes just to the left of the center of the intersection. If the light is red, the driver must stop clearly behind the crosswalk line.

1. Enters intersection in the center of the left-most lane of a 4 (or more) lane street, or center of lane if single lane in each direction, and remains in the center until turning begins.

2. Begins turning when one-third to one-half of the way into the intersection:-

3. If the car must stop for oncoming traffic in the intersection, the wheels must remain straight until the turn is started. (After stop, if vision is blocked by a lefi-turning truck, the driver may move forward into the turn but if he enters the oncoming travel lane and forces vehicles to compensate, examiner will score Hazard or Instructor Control and coder should underline $\mathbf{P}$.)

4. Passes just to left of center of the intersection.

5. Completes turn in center of the left-most lane of intersecting street". (If that lane is blocked or otherwise inaccessible, for example, a roadwork crew or a disabled vehicle, and the driver selects another lane, the examiner will code as Hazard or Instructor Control (see Examiner's Manual) and coder should underline P.)

6. Stops well behind nearest crosswalk line if light is red.

The driver will decelerate smoothly before the turn and accelerate smoothly when clearing the intersection. Failure to accelerate promptly is a frequent error of novice drivers.

1. Decelerates smoothly, coming to a stop or near stop if there is traffic. At no time should the tires squeal or the passengers be thrown:

$=2$. Accelerates smoothly, soon afier he passes the one-third mark in the turn, steadily increasing to traffic speed.

3. Adjusts speed so that vehicle does not interfere with oncoming traffic.

4. Accelerates promptly; if driver hesitates or starts and stops without cause, speed is coded $\mathbf{X}$.

The driver will control his speed before the tum by braking to $10-12 \mathrm{mph}$ one car length from the comer. He will accelerate smoothly two-thirds of the way through the turn. (Driver must approach between 20 and $25 \mathrm{mph}$ ).

1. Before the turn, brakes to $10-12 \mathrm{mph}$.

2. Two-thirds of the way through the turn, accelerates smoothly out of the turn.

3. Accelerates promptly; if driver hesitates or starts and stops without cause, speed is coded $\mathrm{X}$.

- For experienced drivers, it is acceptable to end in any open lane if it is safe to do so. This is not acceptable for novice drivers. 
Left Tuim

Observing

Traffic (O)

High and Medium

Density Traffic

Correct Response

Left Turn

Observing Traffic (O)

Low Density

Correct Response

Right Turn

Approach (A)

High or Medium

Density Traffic

Correct Response

Right Tum

Path (P)

High and Medium

Density Traffic

Correct Response
The driver will scan the intersection for potential conflicts with vehicles and pedestrians.

1. Observes left and right, checks for a gap (looks forward), checks right again and rechecks turn path as he turns.

2. Must not look at any spot longer than 2 seconds (count of 1001,1002 ) unless he must stop. If so, rechecks.

Scans the intersection for potential conflicts with vehicles and pedestrians.

1. Before entering the intersection, looks left, right, and left, in that order. Turns his head far enough to detect pedestrians on the near left and right comers (about $75^{\circ}$ ). (Note: forward scan in light traffic will not be noticeable.)

2. Does not look at any spot longer than 2 seconds (1001, 1002 count) unless there is traffic and the driver's vehicle is stopped.

The driver will steer to the right side of the right-most lane when making a speed reduction for the right turn. This lane is defined as the approach lane. The lateral position of the vehicle in the approach lane is 2 to 3 feet from the curb. If the car must move laterally at least one car width to enter the approach lane, the driver will signal before changing lanes.

(If the local ordinance requires that cars not invade the bike lane, driver may move over to within 3 feet of the curb only when he is one car length from the corner.) When entering the approach lane, the driver must make a timing judgment. By moving or signaling too early he may mislead other drivers to believe that he intends to tum at an earlier location and by moving too late he may risk a bazardous lane change by executing a hurried signal and blind spot check. Driver must stop clearly behind crosswalk when the light is red.

1. If the car must move laterally one car width to enter approach lane:

A. Signals before moving over.

B. If there is a bike lane, driver may stay outside it or move to within 3 feet of curb no more than one car length from comer.

C. Enters the approach lane before vehicle is 4 car lengths from the intersection but not before 10 car lengths.

2. If there is no approach lane, or after approach:

A. Moves to the right to within 3 feet of the curb, to permit following traffic to continue without slowing for the driver's vehicle.

B. Stops well behind nearest crosswalk line if light is red.

C. Signals $\mathbf{1 0 0}$ feet from intersection unless driveway intervenes (when he waits until beyond driveway.)

After the approach, the driver will follow a standard path when turning right.

1. Begins turning - the driver will begin turning the steering wheel when his front wheels are lined up with the beginning of the bend in the curb.

2. Completes turn - the driver must enter the middle of the right-most lane of traffic in a smooth turn. There should be no sudden correction of course. (If that lane is blocked and the driver selects another lane, the examiner will code as Hazard or Instructor Control and coder should underline P.) 
Right Tum

Path (P)

Low Density

Traffic

Correct Response

Right Tum

Speed Control (S)

High and Medium

Density Traffic .

Correct Response

Right Turn

Speed Control (S)

Low Density

Traffic

Correct Response

Right Turn

Observing (O)

High. Mèdium \&

Low Density Traffic

Correct Response
The driver must select a path that will allow him to start his turn two to three feet from the curb and finish his turn in the middle of the right half of the street.

1. Begins turning when vehicle is two to three feet from the curb and front wheels are even with the beginning of the bend of the curb.

2. Turns steering wheel smoothly and follows a smooth curve to the center of the right lane on the new street.

The driver will decelerate smoothly before the turn and accelerate smoothly when clearing the intersection.

1. Decelerates smoothly before the turn to $8-10 \mathrm{mph}$, or less if traffic warrants:

2. Accelerates smoothly two-thirds of the way through the turn, increasing speed quickly enough so that following traffic in new path would not have to slow down or change lanes for-driver's vehicle. (The coder must turn around and = observe traffic.)

3. Adjusts speed to flow of traffic.

4. Accelerates promptly; if driver hesitates or starts and stops without cause, speed is coded X.

Driver must control his speed by braking before the turn and resuming normal speed after completing his turn.

1. Before one car length from the corner, brakes to a speed of 8 to $10 \mathrm{mph}$.

2. Accelerates smoothly as soon as driver is two-thirds of the way through the turn.

3. Accelerates promptly; if driver hesitates or starts and stops withour cause, speed is coded X.

Driver will scan the intersection for potential conflicts with vehicles and pedestrians prior to initiating turn.

1. Tums head to the right and looks for pedestrians or vehicles that could block intended path. If a pedestrian is on right near corner, head movement must indicate check of the spot (75 head movement).

2. Tums head to the left and looks for approaching cross traffic.

3. Turns head to the right again and looks at turn path just prior to the execution of the turn.

Order of checks is imporant. (Note: forward scan will usually not be noticeable.)

4. Completes these checks quickly and does not look for more than 2 seconds (count of 1001,1002$)$ at any spot unless there is traffic and the driver's vehicle is stopped. 
Lanè Change

Path (P)

High or Medium

Density Traffic

Correct Response

Lane Change

Speed (S)

High or Medium

Density Traffic

Correct Response

Lane Change

Observing Traffic ( 0 )

High or Medium

Density Traffic

Correct. Response

Proceeding Through

- Intersection

Observing Traffic ( 0 )

Correct Response
The driver must maintain a straight lane position during scanning procedures (mirrors and blind spot). He must signal before he moves and cancel after the move is completed. The driver then steers smoothly with a gradual angular movement into the middle of the new lane without swerving to either side of the lane.

1. Maintains straight path while scanning.

2. Signals before the lane change.

3. Steers smoothly with gradual angular movement.

4. Straightens vehicle in new lane.

5. Cancels a signal.

Driver will decrease, increase, or maintain speed according to traffic conditions.

1. If flow of traffic of new lane is faster, increases speed when entering new lane.

2. If flow of traffic of new lane is slower, decreases speed when entering new lane.

3. If flow of traffic of new lane is of equal speed, maintains speed when entering new lane.

The driver will scan one or both mirrors, as appropriate, and check blind spot before changing lanes. He will observe traffic ahead in both present and intended lanes for possible slowing. Lane change (right or left)) is necessarily indicated on the scoring sheet at specific locations but refers to the entire segment of the route between two turns. If two lane change arrows occur within a segment (between tums), both of them should be coded whenever they occur. Ignore aborted lane changes. (If they are dangerous, the examiner will code hazard, and mark it wrong.)

1. Checks traffic ahead in present and intended lanes.

2. Checks rear view mirror.

3. Checks outside mirror for left hand change.

4. Checks blind spot by turning the head briefly (appr. 1 second: if the coder counts 1002 , it is too long) in the direction of the lane change and then before steering into the new lane, again checks forward traffic. Driver must not look through back window when checking blind spot. If he does, check is wrong (X).

The driver will scan the intersection before entering.

1. The driver will look left and right before entering a four-way controlled intersection.

2. At a four-way uncontrolled blind intersection, the driver will look left and right before entering the intersection. If blind at that point for less than 200 yards, checks left again.

3. At a blind tee intersection, the driver will look briefly in the direction of the adjoining street.

4. For all checks at intersections, the driver will not look in any direction for more than 2 seconds (count of 1001, 1002) at any spot unless there is traffic and the driver's vehicle is stopped. 
Proceeding Through

Intersection

Speed (S)

Low Traffic Dénsity

Correct Response

Normal Transit

Between Intersections

Speed Control (S)

High-medium or Low

- Traffic Density

Correct Response

Following Distance

(F)

High Traffic Density

Correct Response

Stop Sign

Limit Line (LL)

Correct Response

Mirror Check

(M)
The driver will reduce speed before entering a four-way blind uncontrolled intersection.

1. Brakes gently, to 10 to $15 \mathrm{mph}$, one car length before the corner and continues to cover brake pedal until the traffic checks have been completed.

2. Does not stop or go slowly once into the intersection.

3. Accelerates through the intersection.

The driver will select the appropriate speed for conditions, being guided by speed limit signs, laws, and traffic conditions.

1. Does not exceed the legal speed limit at any time between the. beginning of the (S) coding and the start of coding the next maneuver.

2. Does not exceed the speed of the traffic flow by more than $4 \mathrm{mph}$.

3. Does not fall below the speed limit by more than $4 \mathrm{mph}$ unless traffic or potential hazards require it.

The driver must maintain at least a 2 -second interval from traffic ahead at all times.

Unless there is a significant amount of traffic, there is no opportunity to observe this. If the situation is favorable, there is ample opportunity to observe following distance in normal transit on high density streets. Following distance can also be coded if the driver comes up on slow traffic or traffic stopped at an intersection. Interrupt assessment of following distance to observe mirror check before driver slows down, but then return to following distance as he comes up on traffic. The driver must anticipate slowing of traffic ahead. He must brake gradually enough that he does not throw passengers forward. He must maintain a 2-second distance at all times. Following distance (F) is necessarily indicated on the scoring sheet at specific locations but refers to the entire segment of the route between two turns. If two F's occur, attempt to code it twice in that segment. Do not code following distances of more than four seconds. If it cannot be coded, underline the F's.

Stop sign for the driver's vehicle, but none for cross traffic. The driver must stop behind the limit line (and then continue when traffic permits). If there is no physical limit line, the stop should be made behind a line connecting the two near comers of the street the vehicle is on. The vehicle must not enter the intersecting street.

1. Stops behind limit line.

The driver will check rear view mirror before any speed reduetion or lateral movement and when approaching a traffic light. The coder must attend to the mirror check as soon as the previous coding has been completed, since driver may check early and, if there is no traffic, may not need to repeat. 


\section{Gap Acceptance \\ (G)}

Correct Response

Pre-operation

(Pre-op)

Correct Response

Shut Down

(S.D.)

Correct Response

Y Turnabout

Low Density Traffic

(residential)

Location (L)

Correct Response

Backing (B)

Correct Response
Gap acceptance is coded on some turns where path and speed on the turn are also called for. The novice should decline the smallest gaps that an experienced driver might accept but should not wait when there is ample time.

1. Accepts a 10-second gap; does not accept a gap smaller than 9 seconds". The coder should check his judgment by counting seconds $(1001,1002$, etc.). If driver accepts too small a gap, code it as X. (Examiner may also code Hazard, $X$, or Instructor Control, I.)

2. Gap is also wrong if there is no traffic and driver does not move promptly.

3. If there is no traffic and the driver moves promptly, underline $\mathrm{G}$ (i.e., a judgment cannot be made).

The driver will perform these pre-operation checks before starting the engine:

1. Adjusts seat.

2. Fastens seat belt and shoulder harness.

3. Checks and insures that all passengers are also buckled up.

4. Adjusts both mirrors correctly. If driver must make head adjustments, or adjusts mirrors later, then go back and mark Pre-op wrong.

The driver will perform these shut-down checks:

1. Puts gear selector lever in Park (or gear shift in neutral).

2. Turns ignition off.

3. Sets parking brake.

Driver will chose a safe location for the $\mathrm{Y}$ turnabout (three-point turnabout).

1. Chooses a point at least 200 feet from any intersection.

2. Does not start a $Y$ turnabout when pedestrians or vehicles are nearby.

3. If there is no safe place to turn and the driver traverses the whole block without antempting to turn, underline Location (L) and Backing (B). (The instructor will tell the driver how to get back on the route.)

Driver will look left, right, and back before moving the car in reverse.

1. Looks left and right before backing.

2. Turns head around and looks straight back over right shoulder when moving the car in reverse. Failure to be looking straight back at all times that the car is moving backwards is disqualifying, except for brief checks left, right, and forward.

- Experience drivers may accept smaller gaps, bur must not throw passengers off balance nor clear on-coming car by less than 4 seconds. 
Translation of the Royal Dutch Tourist Association's original "Verslag Rijraardigheidsrit".

The following categories did not apply to the driving test and were dropped from the scoring sheet: "Condition of the Car" (Category I.01), "Railway Level Crossing" (Category III.16), and several Special Maneuvers

(Categories V.19 to V.26, V.28 to V.30).

\section{VEHICLE CHECKS}

\section{Preparing to drive off}

02-1 Passing in front of car and observing traffic

02-2 Clear view and clean windows

02-3. Position of driver's seat

02-4 Instrument check

02-5 Starting the engine

02-6 Use of safety belt(s)

02-7 Checking mirrors

02-8 Proper use of lights

\section{Driving of}

03-1 Checking position of hand-brake

03-2 Looking ahead, to the side and to the rear before driving off

03-3 Selecting correct position in traffic lane

03-4 Adapting speed immediately to traffic flow

03-5 Re-checking mirrors

\section{HANDLING OF VEHCLE}

04 Manner of sitting behind the wheel, and steering

04-1 Manner of sitting behind the wheel when driving

04-2 Position of hands on the wheel

04-3 Steering through curves

04-4 Position of head whilst talking to passengers

05 Handling of controls

05-1 Accelerator

05-2 Foot-brake

05-3 Hand-brake

05-4 Clutch-pedal

05-5 Gear-lever
06 Speed control, deceleration and stopping

06-1 Choice of speed in view of circumstances

06-2 Use of accelerator and brake

06-3 Use of mirrors before and during deceleration

06-4 Indication of deceleration in good time by . use of stop-lights

06-5 Correct sequence of maneuvers for deceleration

06-6 Declutch at the correct stage and put gearlever in neutral

06-7 Come to a stop smoothly

06-8. Clutch not depressed whilst waiting

o7 Taking corners

07-1 Safe starting speed

07-2 Selecting correct gear before entering corner

07-3 Without slipping clutch or foot on the clutchpedal

07-4 No braking in corner

07-5 No free-wheeling

07-6 Correct driving line

\section{ACTION IN TRAFFC}

08 Driving straight

08-1 Keeping to the right

08-2 Adapting speed to that of other similar traffic

08-3 Looking into side streets

08-4 Taking into account blind spots caused by car design and passengers

08-5 Taking into account limitation of mirrors.

08-6 Keeping distance from traffic in front

08-7 Driving in offset position with regard to preceding vehicle

08-8 Pedestrian crossings

08-9 Watching for pedestrians crossing the road (at other places than at zebra crossings) 
09 Behavior at or near crossroads

09-1 Judging the situation beforehand

09-2 Behavior at the approach to traffic lights

09-3 Driving in traffic lanes marked with arrows, and according to other indications on road surface

09-4 Taking position in traffic lanes marked with arrows

09-5 Bicycle and bus lanes

09-6 Consideration of other drivers

09-7 Complying with priority rules

10 Right-hand turn

10-1 Taking position in good time when filtering (selection of correct lane)

10-2 Looking behind and to the right

10-3. Switching on direction indicators

10-4 Positive filtering and in good time, adapting speed

10-5 Looking over right shoulder

10-6. Not impeding traffic that continues straight ahead

10-7 Final check

10-8 Taking corner as closely as possible

\section{Left-hand turn}

11-1 Taking position in good time when filtering (selection of correct lane)

11-2 Looking to the rear, rear left and left

11-3 Switching on direction indicators

11-4 Positive filtering and in good time, adapting speed

11-5 Not impeding traffic that continues straight ahead

11-6 Correct timing of wheel tuming and position on wide crossings

11-7 Final check

11-8 Taking a sufficiently wide comer

\section{Overtaking}

12-1 Judging traffic flow correctly and in time (oncoming, behind and to the lefi)

12-2 Switching on direction indicators

12-3 Moving out smoothly and in good time

12-4 Canceling direction indicators

12-5 Observing vehicle that will be overtaken

12-6 Overaking quickly and safely

12-7 Keeping well clear of overtaken cyclists

12-8 Revering to the right

12-9 Overaking on the right when permitted
13 Traffic lane technique

13-1 Keeping well within lane

13-2 Checking traffic before leaving lane

13-3 Changing traffic lanes one at a time

13-4 Driving straight, avoiding minor deviations

13-5 Driving in lines abreast

13-6 Overhead traffic lane control

14 Driving on roundabouts

14-1 Observation (dividing attention)

14-2 Using direction indicators during the entire maneuver

14-3 Correct positioning for turning in good time.

14-4 Selecting and driving in the correct traffic lane

14-5 Weaving out of the traffic flow correctly and in good time

15 Driving on highways

15-1 Using acceleration lane, observing traffic on highway

15-2 Choice of speed and lane

15-3 Correct distance to vehicle in front

15-4 Stopping and parking in emergency stopping lane (if permitted)

15-5 Leaving emergency stopping lane

15-6 Use of lay-bys

15-7 Behavior at approach to intersections

15-8 Use of deceleration lane

IV OBSERVATION AND UNDERSTANDING OF TRAFFIC

\section{Observation}

17-1 Observation technique

17-2 Observation of overall picrure of traffic, road and surroundings

17-3 Conscious observation of traffic signs

$17-4$ Use of direction signs

18 Understanding of traffic

18-1 Anticipation

18-2 Making allowance for any traffic situations that may occur

18-3 Reactions to observations made

$18-4$ Strategy 


\section{SPECIAL MANEUVERS}

\section{Turning}

27-1 Stopping at the correct place

27-2 Driving slowly, while turning steering wheel rapidly; no use of steering wheel while stopped

27-3 Looking alongside the car when approaching the kerb

27-4 Observing traffic during the entire maneuver 